\title{
Compendium of Instrumentation Whitepapers on Frontier Physics Needs for Snowmass 2013
}

September 24, 2013

\section{Conveners: M. Demarteau, R. Lipton, H. Nicholson, I. Shipsey}

A. Albayrak-Yetkin, J. Alexander, M. Artuso, R. Ball, M. Battaglia, C. Bebek, J. Beene, Y. Benhammou, E. Bentefour, M. Bergevin, A. Bernstein, B. Bilki, E. Blucher, G. Bolla, D. Bortoletto, N. Bowden, G. Brooijmans, K. Byrum, G. Cancelo, C. Chang, J. Chapman, D. Christian, W. Cooper, J. Corso, C. Da Via, S. Dazeley, P. Debbins, G. Deptuch, S. Dhawan, V. Di Benedetto, S. Dye, J. Estrada, H. Evans, E. Etzion, J. Fast, C. Ferretti, B. Fleming, K. Francis, P. Friedman, H. Frisch, M. Garcia-Sciveres, C. Gatto, G. Geronimo, G. Gilchriese, C. Grant, A. Grillo, E. Grunendahl, P. Gorham, G. Gutierrez, C. Haber, G. Haller, C. Hast, U. Heintz, D. Hitlin, C. Hogan, M. Hohlmann, G. Jennings, M. Johnson, H. Kagan, C. Kenney, R. Khanna, V. Khristenko, F. Krennrich, J. Learned, D. Levin, R. Lipton, T. Liu, D.

Lissauer, D. Lynn, D. MacFarlane, S. Majewski, J. Maricic, P. Marleau, A. Mazzacane, A. Mestvirisvilli, N. Mokhov, M. Moshe, M. Newcomer, M.

Narian, K. Nishimura, D. Nygren, E. Oberla, Y. Onel, A. Para, S. Parker, V. Polychronakos, E. Ramberg, B. Rebel, J. Repond, D. Reyna, A. Ronzhin, R. Rusack, A. Ryd, H. Sadrozinski, C. Sanzeni, S. Seidel, A. Seiden, I. Schmidt, A. Shenai, Y. Silver, W. Smith, A. Sonnenschein, D. Southwick, L. Spigel, M. Stanitzki, S. Striganov, D. Su, R. Sumner, R. Svoboda, M. Sweany, R. Tayloe, N. Terentiev, J. Thom, W. Trischuk, M. Vagins, K. Van Bibber, G. Varner, R. Varner, J. Va'vra, H. Von der Lippe, R. Wagner, C. Weaverdyck, H. Wenzel, M. Wetstein, R. Wigmans, D. Winn, A. White, L. Xia, Z. Ye, M. Yeh, T. Yetkin, J. Yoo, J. Yu, B. Zhou, R. Zhu

\section{Contents:}

\section{Energy Frontier:}

- Operation of Collider Experiments at High Luminosity 
- Level 1 Track Triggers at HL-LHC

- Tracking and Vertex Detectors for a Muon Collider

- Triggers for hadron colliders at the energy frontier.

- ATLAS Upgrade Instrumentation

- Instrumentation for the Energy Frontier

- Particle Flow Calorimetry for CMS

- Noble Liquid Calorimeters

- Hadronic dual-readout calorimetry for high energy colliders

- Another Detector for the International Linear Collider

- $\mathrm{e}^{+} \mathrm{e}^{-}$Linear Colliders Detector Requirements and Limitations

intensity Frontier

- Electromagnetic Calorimetry in Project X Experiments The Project X Physics Study

- Intensity Frontier Instrumentation

- Project X Physics Study Calorimetry Report

- Project X Physics Study Tracking Report

- The LHCb Upgrade

- Neutrino Detectors Working Group Summary

- Advanced Water Cherenkov R\&D for WATCHMAN

- Liquid Argon Time Projection Chamber (LArTPC)

- Liquid Scintillator Instrumentation for Physics Frontiers

Cosmic Frontier

- A readout architecture for 100,000 pixel Microwave Kinetic Inductance Detector array

- Instrumentation for New Measurements of the Cosmic Microwave Background polarization

- Future Atmospheric and Water Cherenkov ?-ray Detectors

- Dark Energy 
- Can Columnar Recombination Provide Directional Sensitivity in WIMP Search?

- Instrumentation Needs for Detection of Ultra-high Energy Neutrinos

- Low Background Materials for Direct Detection of Dark Matter

- Physics Motivation for WIMP Dark Matter Directional Detection

- Solid Xenon R\&D at Fermilab

- Ultra High Energy Neutrinos

- Instrumentation Frontier: Direct Detection of WIMPs

- $\mathrm{nEXO}$ detector $\mathrm{R} \& \mathrm{D}$

- Large Arrays of Air Cherenkov Detectors

- Applications of Laser Interferometry in Fundamental Physics Experiments 
Physics Driver: Operation of Collider Experiments at High Luminosity

Time Frame: Medium (2023)

Physics Justification: Experiments running at the HL-LHC will need to operate at a luminosity of $5 \times 10^{34}$ corresponding to $\sim 140$ interactions per $25 \mathrm{~ns}$ crossing. These experiments hope to integrate $3000 \mathrm{fb}-1$ of luminosity. Physics goals include a set of precise measurements of the Higgs branching ratios, search for new physics including supersymmetry and extra dimensions, and measurement of WW scattering. Experiments hope to maintain at least the current values of resolution for tracking and calorimetry and maintain the ability of the triggers to select physics events.

Technical limitations: There are a number of technical challenges to maintaining experimental capabilities at high luminosity. 1) Detectors for forward calorimetry, tracking, and vertexing are not currently sufficiently radiation hard. 2) Level 1 triggers will require information from the trackers based either on region of interest or stand-alone tracking to reduce the trigger rate to acceptable levels. 3) Forward detectors with $\sim 100$ ps time resolution are required to isolate the primary vertex $\mathrm{Z}$ region. 4) Support structures and associated power delivery and cooling systems are currently too massive. This problem will increase with the increasing power density due to pixelization of the tracker and higher data bandwidth. 5) Triggers must be much more selective. This will require increasing L1 latency and data bandwidth.

Technical Capabilities: 1) Study of new detector materials and processes such as diamond and 3D sensors. 2) Develop electronics based on Through-Silicon-Via technology (TSV) to allow the development of trigger layers capable of momentum filtering 3) Study radiation hard detectors and electronics capable of fast timing. 4) Studies of low mass mechanics, $\mathrm{CO}_{2}$ cooling, foamed materials, and high efficiency DC-DC conversion. 5) Development of new DAQ technologies based on ATCA and uTCA, development of low power high speed radiation hard data transmission. 6) Continued development of ASIC technologies.

Key R\&D Directions: The US has had a leading role in the construction and physics analysis of the LHC experiments. It has pioneered 3D and diamond detectors, TSV based electronics, triggering technologies, ASIC development, mechanics and radiation hard silicon technology. Suggested key areas of R\&D with broad impact are:

$\checkmark$ Radiation hard crystal calorimetry

$\checkmark$ ATCA-based novel data acquisition structures

$\checkmark$ 3D silicon technology

$\checkmark$ Low-mass tracking and vertex detectors 
Physics Driver: Level 1 Track Triggers at HL-LHC

Time Frame: Medium (2023)

Physics Justification: The major physics goals of HL-LHC including Higgs decay studies and searches for new physics require that the experiments perform as well, or better then, the current generation. To achieve this CMS and ATLAS will have to upgrade their major subsystems to cope with the higher rates. It is expected that the standard suite of Level 1 muon triggers for CMS will saturate almost independent of threshold. Information from the tracker currently utilized at Level 2 will be needed to reduce trigger rates enough to satisfy Level 1 bandwidth constraints. Availability of tracks of momentum above $2 \mathrm{GeV} / \mathrm{c}$ at less then 3 microseconds after the crossing will enable matching to electron and muon candidates, track isolation, extrapolation to the primary vertex, region of interest readout of the pixels, and possible implementation of PFA type algorithms at Level 1 . The Level 1 tracks must have sufficient $\mathrm{z}$ resolution to remove most of the backgrounds from the $\sim 140$ background interactions.

Technical Requirements: The current designs are based on modules which include pairs of silicon sensors separated by $\sim 1 \mathrm{~mm}$. Hits from these sensors are correlated to provide momentum filtered track stubs. The top tier has long $(1-2.5 \mathrm{~cm})$ strips and the bottom tier is pixelated with short $(\sim 1 \mathrm{~mm})$ strips to provide track $\mathrm{z}$ resolution. There are two candidate tracker layouts, the first based on a hierarchical track-finding design that forms mini vectors (tracklets) from pairs of stubs and extrapolates those tracklets to other layers to form tracks. This design appears capable of delivering a full suite of tracks of $\mathrm{Pt}>2.2 \mathrm{GeV}$ within 2.5 microseconds. The second candidate design utilizes a more conventional disk/barrel geometry and content addressable memory-based track finding.

Technical Capabilities: Modules require unprecedented levels of connectivity to provide correlation between the long and short strip tiers to find stubs. Three dimensional (vertically integrated) electronics which incorporate through-silicon-vias enable the inter-tier communication necessary for hit correlation over a large area module. This technology is a subject of intense development in the semiconductor industry and initial commercial applications are just appearing. 3D enables direct communication of hits between chips along $\mathrm{z}$ to allow for stub formation independent of track dip angle.

The disk/barrel design requires the utilization of Content Addressable Memories (CAMs) which must be both faster and accommodate many more patterns than the implementations in CDF and ATLAS.

Key R\&D Directions: There is an active program that is exploring both via first and via last 3D technologies. Commercial two-tier 0.13 micron via-first 3D chips have been produced and tested as part of a Fermilab-sponsored 3D multiproject run. There is also a collaboration with CERN on the simpler but more generally applicable via-last technology. These technologies correspond to larger $(10 \times 10 \mathrm{~cm})$ and smaller $(5 \times 10 \mathrm{~cm})$ module designs. There is also a current program to explore the use of $3 \mathrm{D}$ or advanced process technology CAM cells for track formation. Initial 2D CAM cells have been submitted for fabrication. 


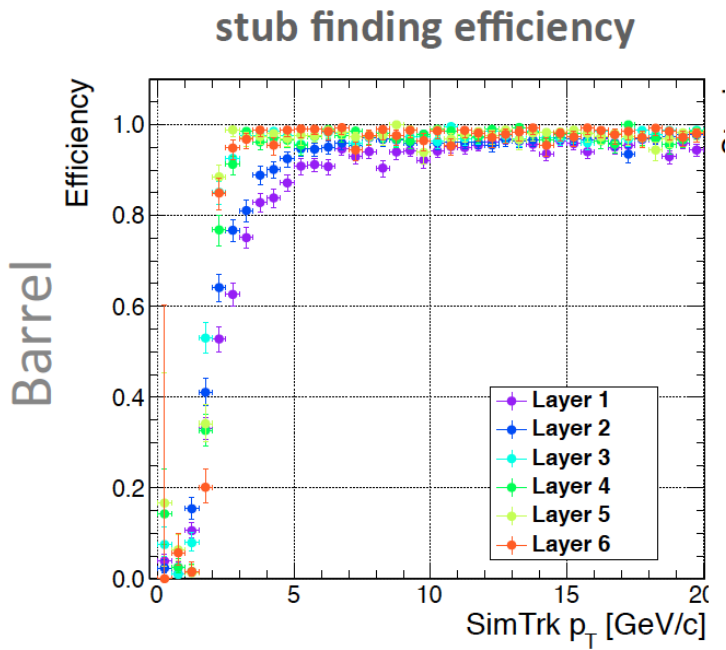

Figure 1. Stub finding efficiency as a function of momentum for various layers in the tracker.

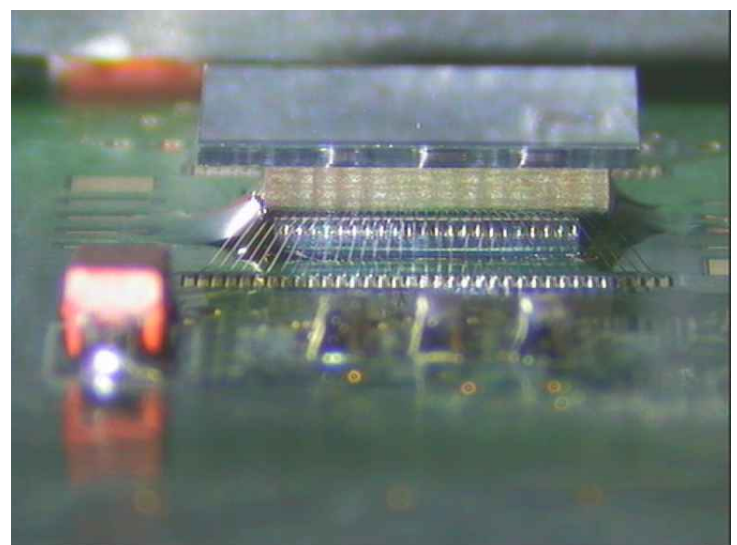

Figure 2.3D chip bonded to a test sensor though a PC board interposer 
Physics Driver: Tracking and Vertex Detectors for a Muon Collider

Time Frame: Intermediate Long (2025-2030)

Authors: V. Di Benedetto, C. Gatto, R. Lipton, A. Mazzacane, N.V. Mokhov, A. Para, S.I. Striganov, N.K. Terentiev, H. Wenzel,

Physics Justification: A Muon Collider is a possible path to a high-energy lepton collider at the Energy Frontier. Alternative technologies with $\mathrm{e}^{+} \mathrm{e}^{-}$colliders, such as CLIC, ILC or a circular machine all suffer from significant limitations for energies in the TeV range. The ILC increases in length in a way that quickly becomes unaffordable. CLIC consumes more than $500 \mathrm{MW}$ at 3 $\mathrm{TeV}$, and circular machines are limited by bremsstrahlung to a few hundred GeV. A Muon Collider scales differently, and can provide a $6 \mathrm{TeV}$ lepton collider that could still fit on the Fermilab site. LHC measurements appear to exclude low energy supersymmetry and a high energy Muon Collider may be necessary to explore new physics discovered at LHC.

In addition to it's high energy scaling the Muon Collider can operate as an s-channel Higgs factory. It should be possible to provide a Muon Beam with a energy spread comparable to the 4.2 MeV Higgs width. This machine can provide a direct measurement of the Higgs width and a measurement of the Higgs mass ten times more precise than $\mathrm{e}^{+} \mathrm{e}^{-}$colliders.

Experiments running at the HL-LHC will need to operate at a luminosity of $5 \times 10^{34}$ corresponding to $\sim 140$ interactions per $25 \mathrm{~ns}$ crossing. These experiments hope to integrate 3000 $\mathrm{fb}^{-1}$ of luminosity. Physics goals include a set of precise measurements of the Higgs branching ratios, search for new physics including supersymmetry and extra dimensions, and measurement of WW scattering. Experiments hope to maintain at least the current values of resolution for tracking and calorimetry and maintain the ability of the triggers to select physics events.

Technical Requirements: The Muon Collider is limited not by bremsstrahlung, but by muon decays. These decays create a background environment that provides the primary challenge for detectors. Detector operation requires careful shielding design, including a tungsten cone that projects at a 10 degree angle from the interaction point. The overall radiation level is comparable to LHC, but is dominated by low energy neutrons and photons and is more uniform over the detector. The region around the beam crossing has limited shielding and the inner radius of the vertex detector must be greater than $6 \mathrm{~cm}$.

MARS studies have shown that these backgrounds are typically very soft and out of time with respect to particles from the beam crossing. Detectors with time resolution of $1 \mathrm{~ns}$ can reduce background levels in the tracker by three orders of magnitude.

Technical Capabilities: For both the tracker and vertex detector requirements include low mass, low power, radiation hardness, and nanosecond level time time resolution. Time resolution is required to reject beam muon decay background. Pixel size in the tracker should be about 1 $\mathrm{mm} \times 100$ microns to insure low occupancy in the high background environment. Pixel size for the vertex detector should be 20 microns $x 20$ microns to provide precise vertexing and $\mathrm{b}$ and $\mathrm{c}$ tagging. Designs have been developed in $65 \mathrm{~nm}$ technology that achieve this time resolution with acceptable power dissipation. 
Key R\&D Directions: Nanosecond time resolution, sensor radiation hardness, low mass, and low power electronics. Possible technologies are 3D integrated sensors and electronics, CMOS active pixel, silicon-on-insulator, and hybrid devices. Of these, 3D technology holds the most promise, since it can utilize radiation hard CMOS combined with radiation hard sensors with active edges. 3D electronics also provides the ability to integrate complex electronics in a very small area by building up the CMOS functionality vertically.

References:

1. R. Lipton, Muon Collider: Plans, Progress and Challenges, DPF 2011, arXiv:1204.3538 [physics.acc-ph]

2. N.K. Terentiev, V. Di Benedetto, C. Gatto, A. Mazzacane, N.V. Mokhov, S.I. Striganov, ILCRoot Tracker and Vertex Detector Hits Response to MARS15 Simulated Backgrounds in the Muon Collider, Physics Procedia, Volume 37, 2012, Pages 104-110

3. Mary Anne Cummings, Stephen Kahn, Accelerator Backgrounds in a Muon Collider, Physics Procedia, Volume 37, 2012, Pages 2114-2122

4. N.V. Mokhov, S.I. Striganov, Detector Backgrounds at Muon Colliders, Physics Procedia, Volume 37, 2012, Pages 2015-2022 
Title: Triggers for hadron colliders at the energy frontier.

Time Frame: Medium (2023)

Physics Justification: The higher luminosity of the HL-LHC will extend the mass reach and significantly increase the sensitivity of searches for new physics. Following the recent discovery of a Higgs boson with a mass of $125 \mathrm{GeV}$, measurement of its self-coupling, observation of its rare decays, and looking for other Higgs bosons will be central to the physics program, as will precision measurements of its mass and tree-level couplings to fermions, $\mathrm{W}$ and $\mathrm{Z}$ bosons. The relatively low mass of the Higgs will require reconstruction and trigger thresholds to remain low with good acceptance over a large $\eta$ range, and the study of vector boson fusion production will require efficient tagging of jets in the forward direction.

Technical Capabilities: The HL-LHC operating scenario is to level the instantaneous luminosity at $5 \times 10^{34} \mathrm{~cm}^{-2} \mathrm{~s}^{-1}$ from a potential peak value of $1 \times 10^{35} \mathrm{~cm}^{-2} \mathrm{~s}^{-1}$ at the beginning of fill, and to provide $250 \mathrm{fb}^{-1}$ per year for about 10 years. Operating the HL-LHC with $25 \mathrm{~ns}$ bunch crossing spacing at at $5 \times 10^{34} \mathrm{~cm}^{-2} \mathrm{~s}^{-1}$ implies a pileup of up to $200 \mathrm{~min}$-bias events/crossing. This is an order of magnitude greater than the LHC design luminosity $\left(10^{34}\right)$ figure of 17 min-bias events per crossing and will degrade all occupancy-dependent trigger algorithms that rely on forms of isolation to identify electrons, muons, taus and missing energy signals. This requires a better performing trigger with additional information, such as tracking data, used to reduce the trigger rates against the much higher backgrounds. The size of regions sampled for trigger decisions will need to shrink to handle the increased backgrounds. Therefore, substantial improvements will be needed for the ATLAS and CMS Trigger systems for HL-LHC. The addition of track information in L1 Trigger algorithms can provide significant improvements, particularly for lepton triggers, and helps with the overall rate problem, although hadronic triggers may still require higher bandwidth. L1 tracking trigger calculations could include the provision of a pixel trigger to identify vertices and the combination of calorimeter and muon trigger information with that of the tracking trigger. The tracking trigger reconstruction of tracks with good efficiency down to a $\mathrm{p}_{\mathrm{T}}$ of $\sim 2.5 \mathrm{GeV}$ or lower would be needed to provide lepton isolation. The new trigger logic must operate in a short time envelope or latency unless substantial front end electronics modifications are made in the ATLAS and CMS Detectors since these trigger calculations may require more latency than available in the front end electronics buffers. The increased selectivity of the better performing L1 trigger and the increased complexity of the higher occupancy events will place a greater burden on the Higher Level Triggers, requiring a substantial increase in processing power.

Technical Requirements: Studies of the hardware capabilities and firmware technology to fully exploit advances in Field Programmable Gate Arrays (FPGAs), including architectures for the large increase in logic cells and high speed and large number of serialization/deserialization stages. Studies of new compact high-density optical connectors and receivers to understand integration into trigger board designs. Studies of Advanced Telecommunications Computing Architecture (ATCA) and Micro-TCA derived from the Advanced Mezzanine Card standard to understand how to leverage high-speed star and mesh backplanes and the control technologies to provide the most effective infrastructure for trigger systems. Studies of alternative processing units for dedicated higher-level trigger calculations such as Graphical Processor Units (GPUs). 
Studies of Higher Level Trigger algorithm code to improve parallelization and thereby speed up CPU time.

Industrial Involvement: Much of the technologies for future triggers are developed by industry. For example, FPGA development is done by Xilinx and Altera. There are a number of vendors supplying optical connectors and receivers, ATCA and MicroTCA crates and GPUs. The trigger research program would need to work with these vendors.

\section{Key Motivations for this Detector R\&D:}

$\checkmark$ Increases physics capabilities of very large detectors at high luminosity.

$\checkmark$ Increases sensitivity to low cross section processes with large backgrounds at high luminosity.

$\checkmark$ Provides increased flexibility to respond to changes in physics priorities and accelerator performance. 


\title{
SNOWMASS WHITEPAPER
}

June 11, 2013

\section{ATLAS Upgrade Instrumentation}

\author{
Gustaaf Brooijmans ${ }^{\mathrm{a}}$, Hal Evans ${ }^{\mathrm{b}}$, Abe Seiden $^{\mathrm{c}}$ \\ ${ }^{\mathrm{a} C o l u m b i a}$ University \\ ${ }^{\mathrm{b}}$ Indiana University \\ ${ }^{\mathrm{c}}$ University of California Santa Cruz
}

\begin{abstract}
Planned upgrades of the LHC over the next decade should allow the machine to operate at a center of mass energy of $14 \mathrm{TeV}$ with instantaneous luminosities in the range $5-7 \times 10^{34} \mathrm{~cm}^{-2} \mathrm{~s}^{-1}$. With these parameters, ATLAS could collect $3,000 \mathrm{fb}^{-1}$ of data in approximately 10 years. However, the conditions under which this data would be acquired are much harsher than those currently encountered at the LHC. For example, the number of proton-proton interactions per bunch crossing will rise from the level of 20-30 per $50 \mathrm{~ns}$ crossing observed in 2012 to 140-200 every $25 \mathrm{~ns}$. In order to deepen our understanding of the newly discovered Higgs boson and to extend our searches for physics beyond that new particle, the ATLAS detector, trigger, and readout will have to undergo significant upgrades. In this whitepaper we describe R\&D necessary for ATLAS to continue to run effectively at the highest luminosities foreseen from the LHC. Emphasis is placed on those R\&D efforts in which US institutions are playing a leading role.
\end{abstract}




\section{Contents}

1 Introduction 2

2 Tracker Upgrade 3

2.1 General Design Goals and R\&D Areas . . . . . . . . . . . . . . . . . . . . . 3

2.2 ATLAS Letter of Intent Tracker . . . . . . . . . . . . . . . . . . . . 4

2.3 Performance Expectations $\ldots \ldots \ldots \ldots \ldots \ldots$

3 Calorimetry $\quad 8$

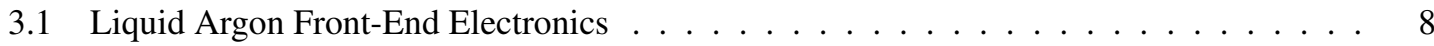

3.2 Tile Calorimeter Front-End Electronics ～. . . . . . . . . . . . . . . . . . . . . 10

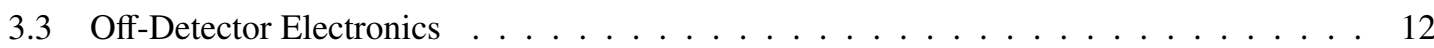

3.4 Summary of Main Calorimeter R\&D Areas . . . . . . . . . . . . . . . 13

4 Trigger and Data Acquisition $\quad 14$

4.1 Physics Motivation . . . . . . . . . . . . . . . . . . . . . . . . . 14

4.2 TDAQ System before Phase-II . . . . . . . . . . . . . . . . . . . . . . . . . 14

4.2.1 Involvement of US Groups in the TDAQ . . . . . . . . . . . . . . . . . . 16

4.3 Phase-II Trigger Architecture . . . . . . . . . . . . . . . . . . . . . 16

4.3.1 Rate Estimates for the Trigger at Phase-II Luminosities . . . . . . . . . . . . . . 16

4.3 .2 Proposed Phase-II Architecture . . . . . . . . . . . . . . . . . . 16

4.4 Calorimeter Trigger . . . . . . . . . . . . . . . . . . . . . . . 17

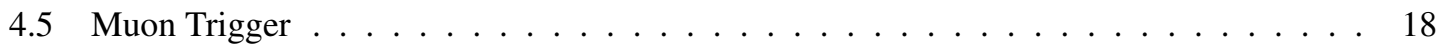

4.6 Level-1 Track Trigger . . . . . . . . . . . . . . . . . . . . . . . . . . . . 19

4.7 Central Trigger System . . . . . . . . . . . . . . . . . . . . 20

4.8 High Level Trigger . . . . . . . . . . . . . . . . . . . . . . 20

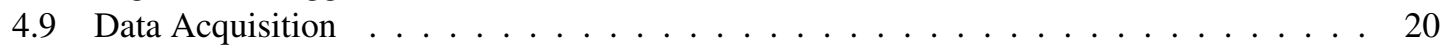

4.10 Summary of Main TDAQ R\&D Areas . . . . . . . . . . . . . . . . . . . 21

5 Conclusion 22 


\section{Introduction}

The LHC is expected to be the world's Energy Frontier machine for at least the next 15 years. Data to be collected will allow much more detailed studies of the Higgs boson, the search for other new phenomena and the study of these if found, and in general push the sensitivity for new physics well into the multi- $\mathrm{TeV}$ range [1]. This physics program provides the challenge of simultaneously looking at many decay and production channels for a $125 \mathrm{GeV}$ particle, resulting in final state particles of moderate tranverse momenta, and also searching for particles at the highest mass scale that can be probed by the accelerator, which can result in very high momentum particles and large missing energy, all with very many superposed pile-up events.

The mass of the Higgs boson is ideal for the study of many decay and production channels, allowing an incisive test of our model for electro-weak symmetry breaking. However, present data samples are quite limited and all channels are still crudely measured. Providing much more precise measurements will require much more data. A major goal of the Phase-II upgrade of the LHC is to provide approximately 300 times as many events in the various Higgs channels as presently exist (and 10 times what will exist prior to the upgrade). This sample will also open up some new channels with small rates to provide a more comprehensive Higgs picture. In addition, to more fully complete the picture of electroweak symmetry breaking, the measurement of $\mathrm{W}-\mathrm{W}$ scattering at the TeV mass scale is very important. Acquiring such an event sample requires accelerator upgrades in luminosity, and detector upgrades that maintain trigger thresholds to enable collection of key events while preserving a high quality detector with broad capabilities to limit systematic errors on the quantities we wish to measure.

The presently planned machine upgrade will be to an instantaneous luminosity of $5-7 \times 10^{34} \mathrm{~cm}^{-2} \mathrm{~s}^{-1}$ and would use luminosity leveling at a $14 \mathrm{TeV}$ center-of-mass energy to provide the largest number of events. The goal is to collect $3,000 \mathrm{fb}^{-1}$ of data in approximately a 10 year time span. The number of interactions will rise from the present 20 to 30 per $50 \mathrm{nsec}$ crossing to at least 140 and possibly 200 every $25 \mathrm{~ns}$.

The Phase-II detector will require fundamental upgrades in the trigger and detector electronics across all systems to maintain thresholds and efficiency, as well as an entirely new tracker to deal with the increased radiation damage, occupancy, and data rates. These improvements will be based on advances in technology over the more than 15 years since the present ATLAS detector was designed, and the vigorous ATLAS upgrade R\&D program now ongoing. The various major parts of the Phase-II upgrade are described below with an emphasis on US efforts. A much more detailed discussion can be found in the ATLAS Phase-II Letter of Intent (LoI) [2] with more background material available in the Phase-I LoI [3]. 


\section{Tracker Upgrade}

\subsection{General Design Goals and R\&D Areas}

The design of the upgraded ATLAS tracker is governed by the physics needs of the experiment and the desire to minimize cost. The design under consideration is based entirely on layers of pixel and strip silicon sensors, which can provide the granularity and radiation hardness required for the tracker. Minimizing cost and material in the tracking volume requires the fewest layers that can provide robust pattern recognition and track parameter measurement. This has been determined by simulations to be four pixel layers surrounded by five double-sided strip layers spread out over the 1 meter radius tracking volume inside the ATLAS solenoidal magnet. Maintaining an occupancy below about $1 \%$ per sensor element allows good matching of strip layers arranged to provide small angle stereo information with few fake tracks. In order to keep the occupancy below $1 \%$, we must reduce each cell size, resulting in an increase in the number of readout channels by an order of magnitude compared to the present ATLAS detector. The inner radius of the less costly strip layers is determined by radiation damage and the desire to have at least half the signal strength from a passing particle, as compared to an unirradiated detector, after collecting $3,000 \mathrm{fb}^{-1}$ of data.

To identify many of the physics signatures, the tracker must provide excellent momentum measurements for energetic leptons and establish that they are in fact isolated. The material in the tracker affects the efficiency and resolution in the detection of isolated electrons, due to bremsstrahlung and nuclear interactions. The performance of $b$-tagging algorithms based on displaced vertices also requires controlling the tails in the impact parameter measurement due to errors in pattern recognition and hadronic interactions within the active volume of the tracker. The upgraded tracker therefore must maintain careful control of the material inside the tracking volume, despite the larger channel count. This has significant implications on the mechanical design of the support structure, the powering scheme, and the cooling. In addition new physics analysis techniques have emerged that place stringent requirements on future trackers. For example, the use of highly boosted jets require that track reconstruction work efficiently in very collimated jets, where particle tracks remain close to each other over long distances. This demands small pixels and thin sensors to avoid large cluster sizes.

An important lesson from the $8 \mathrm{TeV}$ run is that tracking can be used to build powerful tools to mitigate the effects of pileup for a wide range of objects measured by other subdetectors. Therefore, another important requirement on the upgraded tracker is the need to provide fast momentum information on tracks to select events of interest at the first trigger level. Since the readout and reconstruction of all tracks for a first level trigger is presently beyond the state-of-the-art, the ATLAS plan is to only readout a fraction of the tracking detector $(\sim 5 \%)$ for participation in the first level trigger, chosen via regions of interest based on information from the calorimeter and muon systems. This requires high-bandwidth frontend and controller chips that allow for readout paths both for triggering and more general readout.

The large integrated fluences for Phase-II will require more radiation hard sensors than in the present ATLAS detector but also at a minimum cost. In the case of pixels the goal has been to develop larger detectors (for example $4 \mathrm{~cm} \times 4 \mathrm{~cm}$ in area) that can be bump bonded to several (for example four) frontend chips, significantly reducing bump bonding costs. For the strip sensors, units that are about $10 \mathrm{~cm} \times$ $10 \mathrm{~cm}$ allow cost minimization with further segmentation provided by reading out shorter strip segments (for example $2.5 \mathrm{~cm}$ length) using hybrids and electronics located on top of the sensor. Maintaining the sensor signal after large fluences requires the collection of electrons. For most of the pixels and all of the strips cost minimization then determines that the sensor be of the n-on-p type. For the two very inner pixel layers, 3-D sensors, where readout collection columns are embedded in the silicon, allow very short collection distances and the highest radiation tolerance. The use of slim-edges for the sensors will avoid having to overlap them to maintain hermetic coverage, reducing material and the number of sensor units.

The issues described above lead to a number of major R\&D areas. US groups have played a large 
role in many of the analogous areas for the present ATLAS detector. Currently, three national lab groups and six university groups are playing a major role in the R\&D program for Phase-II. Some primary areas in that program are:

- Development of minimum cost sensor assemblies in n-on-p technology, aimed at both the strip detector and outer pixel layers.

- Optimizing 3-D sensors for the two inner pixel layers. Other options for this part of the detector are diamond sensors or planar detectors that can run at rather high voltages. In addition new approaches, for example CMOS sensors, could have a large impact but require significantly more development and testing.

- Developing slim-edge sensors.

- Developing pixel frontend chips, with those for the inner two layers being most challenging and requiring very high performance CMOS technology.

- Development of strip front-end electronics, including features for first level triggering.

- Developing very light-weight supports for mass minimization.

- Developing novel powering schemes to save on cabling, mass, and cost.

- Developing chips which provide for interfaces to the data acquisition and also to the powering system.

- Developing fabrication schemes that minimize assembly time and complexity.

- Developing cooling systems with minimum mass, which are robust and allow running well below room temperature.

An additional area of importance is the optical fiber system, the optical transmission components, and read-out-drivers. This is an area where options from industry develop rapidly over time and we are mainly following options available commercially. However, high bandwidth readout is expected to be of great importance in moving the information from the sensors to a region at larger radius.

All of the newly developed items must be tested for radiation hardness (e.g. signal properties for sensors; speed, gain changes, and single event upset for electronics), which leads to a very demanding and time-consuming test program.

The next section presents the performance of the tracker we expect to be able to build given several more years of $R \& D$. This assumes that the $R \& D$ projects listed above converge to construction-ready status over that time period. The R\&D over the past few years and performance of the present detector provide some confidence that this can be achieved given adequate funding.

\subsection{ATLAS Letter of Intent Tracker}

Figure 1 shows the tracker that has been simulated and on which the following performance plots are based. It consists of four pixel layers and five double-sided strip layers in a long barrel, and a collection of disk layers to cover more forward rapidities. The use of a barrel that is much longer than in the present ATLAS detector leads to a reduction in material as well as pushing material to larger rapidities. The layout has been adjusted to maintain 14 precision measurements on nearly all tracks, required for good pattern recognition. Figure 2 (a) shows the number of hits on tracks originating from the origin and also from $15 \mathrm{~cm}$ along the beam-line, indicating the excellent coverage. Figure 2 (b) shows the material traversed (in radiation lengths) by a high momentum track as a function of rapidity. The different sources 
of material are indicated. The layout arranges for a minimum amount of material at rapidity below 1.6, with most of the services beyond this. Figure 3 shows the occupancy expected in the tracker for each barrel layer for the case of 200 interactions in a crossing.

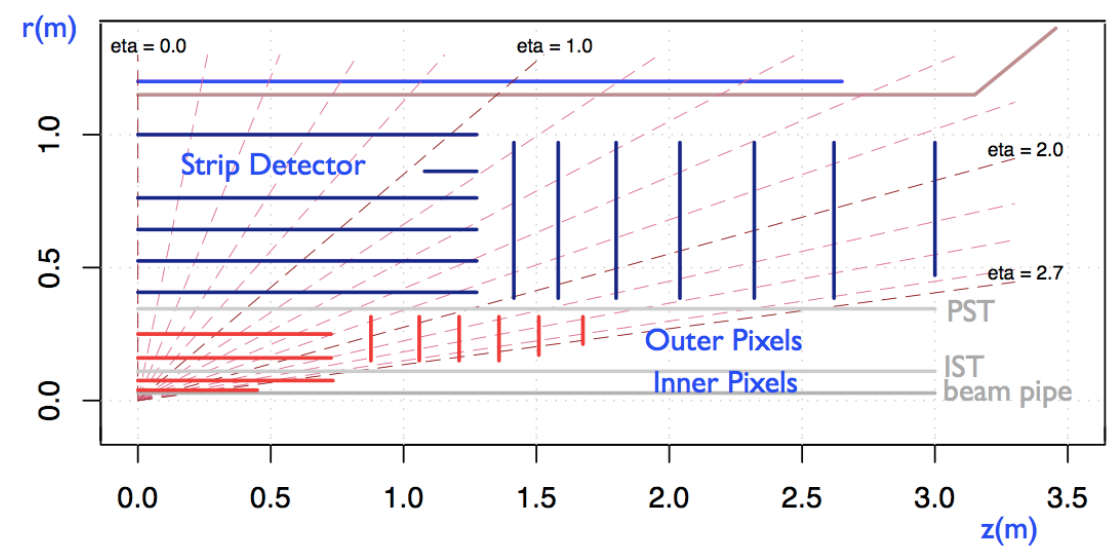

Figure 1: Diagram showing the layout of the tracker presented by ATLAS in the Phase-II LoI.

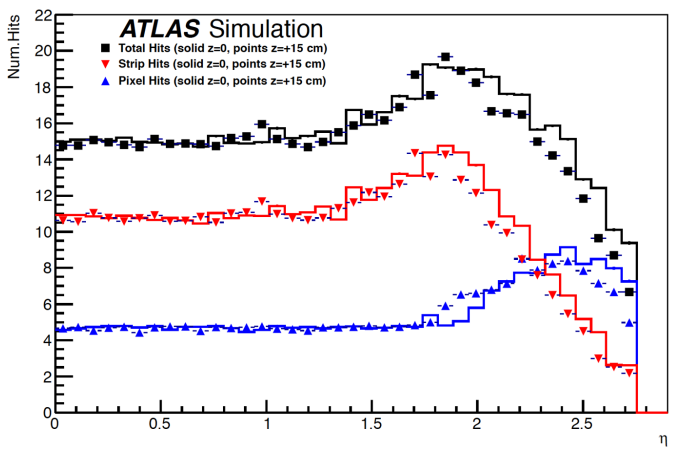

(a)

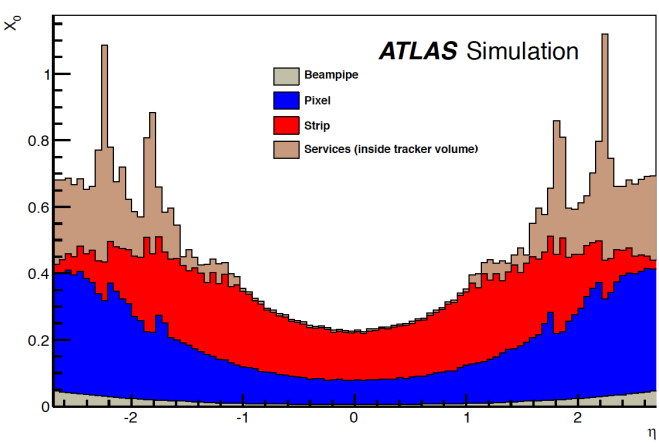

(b)

Figure 2: (a) Number of hits on a high momentum track originating from the origin (solid) and $15 \mathrm{~cm}$ from interaction point (points), broken down between pixel hits, hits on strip detector, and total hits. (b) The material traversed by tracks from the origin, broken down by source of material.

\subsection{Performance Expectations}

The tracker is designed for physics at up to 200 events per crossing. We present below some of the performance expectations. Figure 4 (a) shows the expected momentum resolution for muons of three different transverse momenta. Despite the discrete layer spacing the tracker achieves a rather smooth distribution versus rapidity and improves on the present tracker. Figure 4 (b) shows the ratio of reconstructed to generated tracks from $t \bar{t}$ events as a function of pileup for two different track selections: requiring track reconstruction with at least 9 hits per track (a), and with at least 11 hits per track (b). The choice of 14 hits allows us to achieve good performance (that is no increase in fake tracks with pileup) with some redundancy, for example with some missing hits (1 pixel layer and 1 double-sided strip layer). Figure 4 (c) 

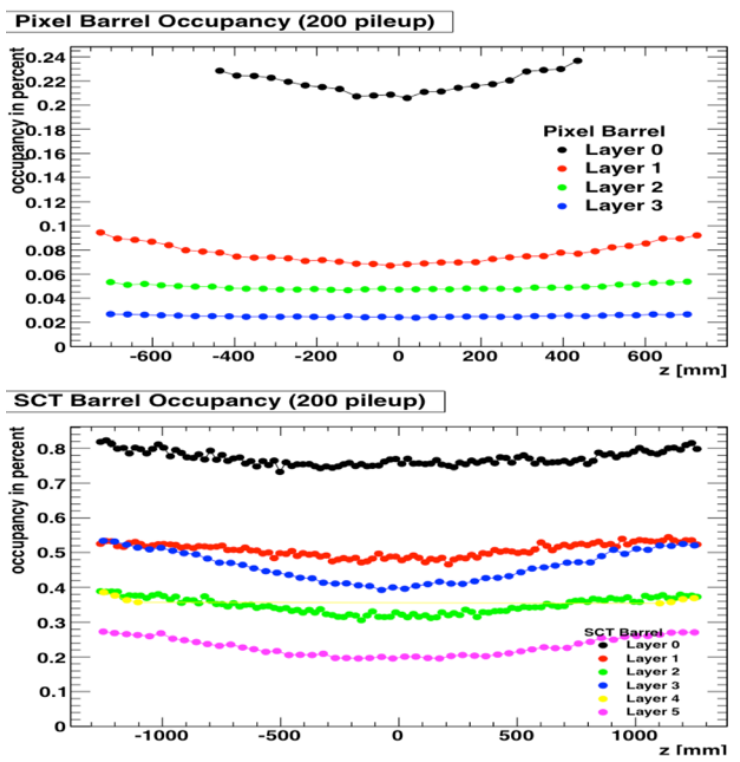

Figure 3: Occupancy expected in the LoI tracker for 200 interactions per crossing.

shows the expected $b$-tagging performance for different levels of pileup as well as a comparison to the expectations for the present tracker, including the new Insertable B-Layer (IBL). The performance for b-tagging of the upgraded tracker (labeled ITK) with 140 events per crossing is approximately as good as that for the present tracker with no extra occupancy. This level of performance is also crucial for reconstructing $\tau$ leptons. 


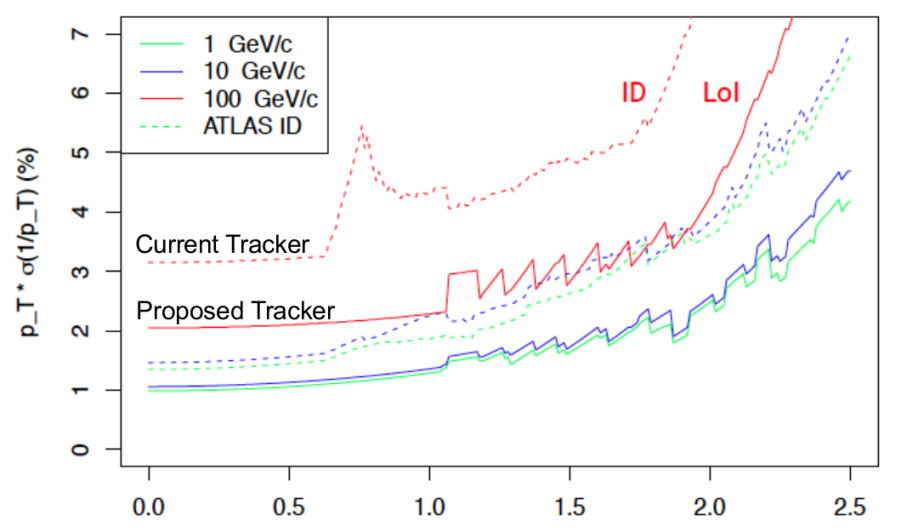

(a)
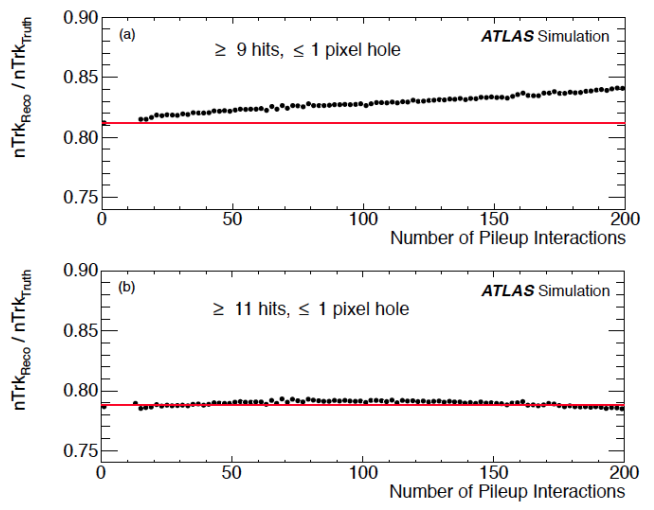

(b)

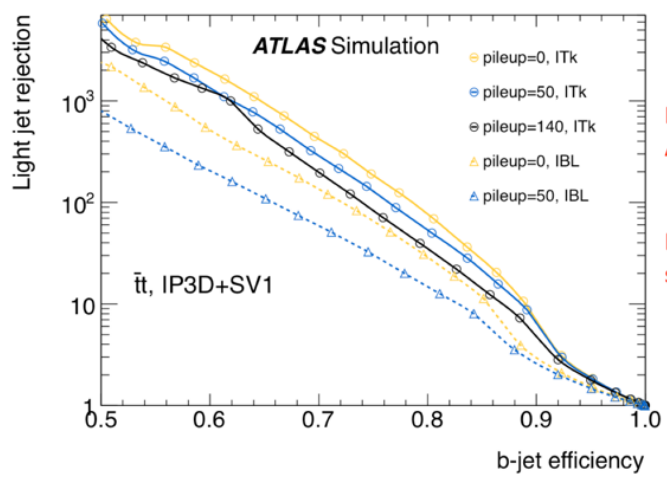

(c)

Figure 4: (a) Predicted momentum resolution for muons as a function of pseudorapidity for the new inner tracker (solid lines) compared with the current tracker (dotted lines) for three different values of the momentum. (b) Ratio of reconstructed to generated tracks from simulated $t \bar{t}$ events at various levels of pileup for two different track selections: requiring track reconstruction with at least 9 hits per track (b-a), and with at least 11 hits per track (b-b). (c) Expected b-tagging performance, the efficiency to tag a $b$-jet and related rejection factor for light-quark jets. 


\section{Calorimetry}

Calorimetry at high-luminosity, high-energy hadron colliders faces a number of major challenges:

- Front-end electronics need to be radiation-tolerant and consume little power while achieving highprecision (typically 16-bit dynamic range) and high data transmission bandwidth. Furthermore, these usually need to be installed on-detector, i.e. in inaccessible locations that impose stringent reliability requirements.

- High radiation levels and particle fluxes, particularly in the forward regions, impose the use of technologies that are simultaneously very radiation tolerant and able to yield high-precision measurements in the presence of high background fluxes.

The value of high-precision calorimetry to accurately measure electrons, photons and hadronic jets has been well established by the discovery of the Higgs boson and e.g. precision measurements in top quark physics [1]. During Phase-II operation, particle fluxes and the average energy deposited in the calorimeters are expected to be typically five to ten times higher than specified in the LHC design values. Under these conditions it is certain that the front-end electronics will have to be replaced. This is due both to radiation damage and the need for ATLAS to upgrade the trigger system, with the latter requiring real-time performance capabilities that the current electronics cannot satisfy.

The ATLAS calorimeters are based on two technologies: liquid argon sampling for the electromagnetic and forward hadronic calorimeters, and scintillating tile sampling for the central hadronic calorimeter. In both cases, the front-end electronics currently send coarse data to the Level-1 trigger system while buffering the precision data for readout on Level-1 accept. Ongoing R\&D work is aimed at exploiting technological progress to send the full precision data to the Level-1 trigger system for all beam bunch crossings.

The US has played a leading role in the development and construction of the initial ATLAS calorimeter electronics: most of the ASICs for the liquid argon front-end electronics were developed in the US, and the 1524 front-end boards were produced in the US. The liquid argon back-end electronics were initially designed in the US. For the TileCal, the US led the front-end electronics design and played a major role in their construction.

\subsection{Liquid Argon Front-End Electronics}

The main architectural difference between the existing readout system and the planned upgrade is the switch from an (analog) on-detector Level-1 pipeline to a free-running design in which signals from all calorimeter cells are digitized at $40 \mathrm{MHz}$ and sent off-detector. This approach effectively removes all constraints imposed by the liquid argon calorimeter (LAr) readout on the trigger system, since full precision, full granularity data will be available and the latency and Level-1 bandwidth will become essentially unlimited. Furthermore, due to its minimal coupling to the trigger system, the new architecture will provide significant flexibility for further evolutions in the ATLAS trigger system or overall detector readout. It will be able to accommodate a trigger system with a low-latency, low-granularity Level-0 trigger stage just as well as a system with a high-latency, full-detector Level-1 trigger, or both. Figure 5 shows the readout architecture for Phase-II.

The front-end boards (FEB) perform analog shaping of the calorimeter signals, then sample the analog waveform at $40 \mathrm{MHz}$ before multiplexing the digitized outputs for transmission over fast optical links. (The LAr trigger digitizer boards perform very similar tasks with somewhat worse precision and coarser granularity to provide a more manageable data volume for the Level-0 trigger.) Each FEB reads out 128 calorimeter channels and transmits data at approximately $100 \mathrm{Gbps}$. A total of 1524 FEBs are needed to read out the full calorimeter. 


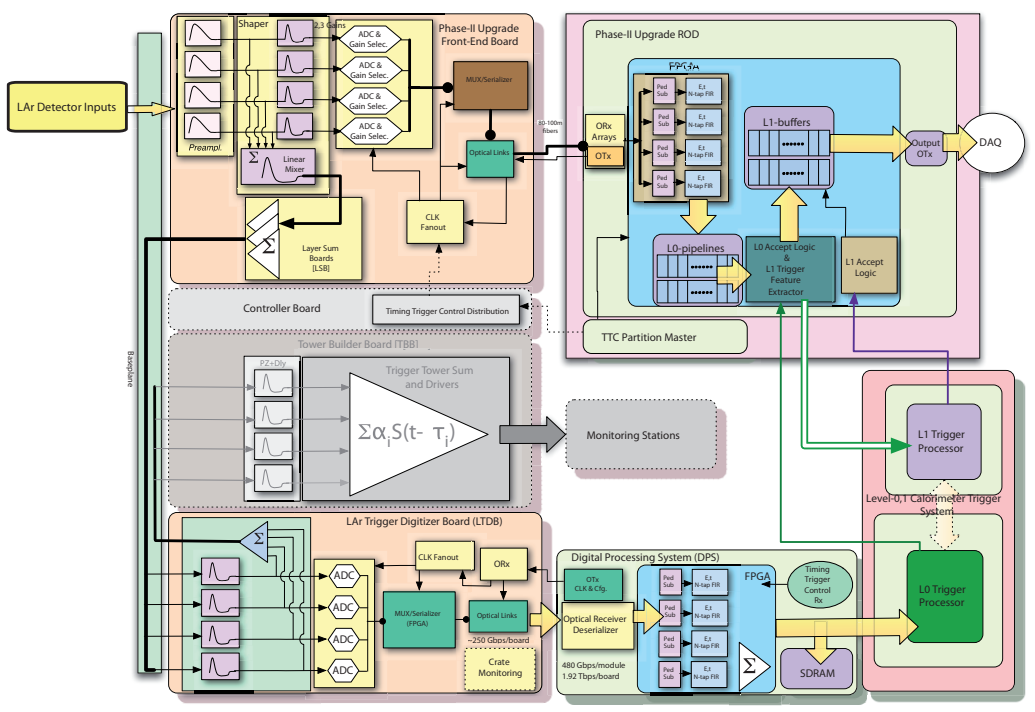

Figure 5: Phase-II readout architecture for the liquid argon calorimeters. The front-end and LAr Trigger Digitizer boards are located on-detector, and send the data over fast optical links to the off-detector ROD and Digital Processing System. The LTDB and DPS will be installed in the Phase-I upgrades in 2018.

The new front-end board architecture is designed to remove the bottlenecks inherent in the current boards by exploiting progress in technology, while maintaining the analog performance: 16-bit dynamic range (currently achieved with three gains at 12 bits each) and coherent noise below $5 \%$ of the incoherent noise level. The main functional blocks are: analog pre-amplification and shaping, production of (summed) analog signals for the LTDB, analog-to-digital conversion of all signals at a rate of $40 \mathrm{MHz}$, multiplexing and serialization of digital data, and transmission over high-speed optical links. Since there will be no Level-1 pipeline, the control logic will be limited to clock distribution and slow controls for configuration and calibration.

US R\&D Efforts address all aspects of the necessary front-end electronics development. The analog preamplification and shaping stages will be integrated in a single ASIC. The Liquid Argon Preamp and Shaper (LAPAS) test-chip, designed by the University of Pennsylvania and Brookhaven National Laboratory, and fabricated in 2009 in IBM's $8 \mathrm{WL}$ SiGe process, validated the design approach of implementing a wide dynamic range single ended preamp followed by low power differential shaping stages with multiple gains to achieve the required 16 bit resolution. An integral non-linearity of less than $0.6 \%$ was demonstrated in both the $1 \times$ and $10 \times$ gain shaping stages and measurements of ionizing radiation indicated robust performance for TID in excess of $1 \mathrm{MRad}$ [4]. Less expensive SiGe process alternatives are currently being explored: IHP SG25H3P and IBM's 7WL.

The requirements on the $\mathrm{ADC}$ are a large dynamic range (16 bits can be achieved using three gains and 12-bit ADCs if needed) and precision (at least 12 bits), low power $(<100 \mathrm{~mW} / \mathrm{channel})$, high speed (40 MSPS), small footprint (128 channels on a $50 \mathrm{~cm}$-wide board) imposing serialized outputs, and substantial radiation tolerance. Many commercial devices meet most of these criteria but are very sensitive to single event upsets (SEUs). Irradiation tests are being performed to verify if recent devices are suitable.

In addition to radiation tolerance, ASIC solutions have lower latency and power consumption. The former aspect is crucial if the signals are to be used in the Level-0 or Level-1 trigger systems. A recent test chip (nevis12, developed at Columbia Universitys Nevis Labs) in IBM CMOS 8RF (130 nm) technology uses four 1.5-bit pipeline stages followed by an 8-bit SAR. The output data are serialized at $640 \mathrm{Mbps}$. 
A predecessor has allowed to demonstrate analog performance of the pipeline part at least equivalent to that of a commercial 12-bit ADC together with excellent radiation tolerance, and early test results from nevis 12 are promising. Full testing will be completed in 2013 to confirm its analog precision, radiation tolerance, and determine the optimal operational parameters. The power consumption is expected to be approximately $40 \mathrm{~mW} / \mathrm{channel}$ (40\% of typical COTS ADCs) and the latency $65 \mathrm{~ns}$ (100 ns less than the typical COTS pipeline ADC). Figure 6 shows a schematic diagram of the nevis 12 chip.

After digitization, the raw data produced on a FEB represents 128 (channels) $\times 14$ bits (including two gain scale bits) $\times$ approximately $40 \mathrm{MHz}$, or about $72 \mathrm{Gbps}$. Conservatively assuming $25 \%$ overhead for control words and encoding, each board needs to multiplex, serialize and transmit $90 \mathrm{Gbps}$ of data, leading to a total data transmission rate of $140 \mathrm{Tbps}$ for the full calorimeter. A radiation-tolerant serializer in $250 \mathrm{~nm}$ silicon-on-sapphire technology able to run at $8 \mathrm{Gbps}$ has already been developed by Southern Methodist University, and with a newer process, speeds of $10 \mathrm{Gbps}$ should be achievable. It should therefore be possible to use a single 12-fiber ribbon to transmit the data produced by each FEB.

Further US ASIC R\&D plans include: the production of a quad-channel 12-bit ADC, a $5.5 \mathrm{Gbps}$ multiplexer and serializer chip and a 5.5 Gbps laser driver chip for the Phase-I upgrades; developing a 16-bit dynamic range (12-bit precision) ADC which could possibly incorporate the pre-amplification and shaping stages; incorporation of the ADC and high speed (5-10 Gbps) output serializers.

An oft-overlooked but crucial issue is the power distribution to the front-end electronics. The powering concept for Phase-II is based on a distributed power architecture with crate-level main converters providing $48 \mathrm{~V}$ and board-level point-of-load converters (POL). Modular main converters with $1.5 \mathrm{~kW}$ per module are under development with conversion efficiencies above $80 \%$. Operation in external fields up to 300 Gauss, as present at the location on the LAr detector, has been successful. POL converters need to operate in even stronger magnetic fields, which prevents the usage of magnetic materials for inductance cores. Several developments of air-core based POLs are currently ongoing at Brookhaven National Laboratory and different technologies, based on SiCMOS and GaN, are under study.

\subsection{Tile Calorimeter Front-End Electronics}

The goals of the Tile Calorimeter Phase-II upgrade are to increase the radiation tolerance of the front-end electronics and to maximize the resolution and configurability of the tower triggers by sending all tile cell data to the sROD off-detector. This is feasible with the advent of $>10 \mathrm{Gbps}$ optical communications.

Achieving these goals requires replacement of all the on-detector electronics as well as the SROD off-detector readout. Preservation of data integrity is achieved in three stages. The first line of defense is achieved by using better radiation tolerant components. Next, there is the creation of redundancy in the on-board electronics so that data is preserved if one power supply or microprocessor fails. Additionally, tile cells are read out by two phototubes sent to separate readout channels. Finally, error correction is employed in the serial communication scheme. An overview of the tile electronics system is shown in the Figure 7.

In the upgraded scheme, the on-detector electronics are housed in a mechanical drawer on which are mounted phototubes, an amplifier/shaper card (which might also digitize the signal), a Main Board which distributes low voltage and control signals, holds the ADCs, and routes data to a high-speed Daughter Board where the error correction and communications processing occurs. Additionally, the drawer houses a High Voltage control card for the phototubes. The Main Board is a complex $69 \mathrm{~cm}$ PCB of 14 layers, currently prototyped by the University of Chicago group. This board must handle all controls, signal digitization, and timing delays.

Integrity of the Low- and High-Voltage systems is paramount to the reliability of the Tile system, and both have been a source of problems in the past. The Argonne group has undertaken to redesign both systems. Their earlier redesign of the low-voltage modules for the current detector reduced noise and increased radiation tolerance by an order of magnitude. 

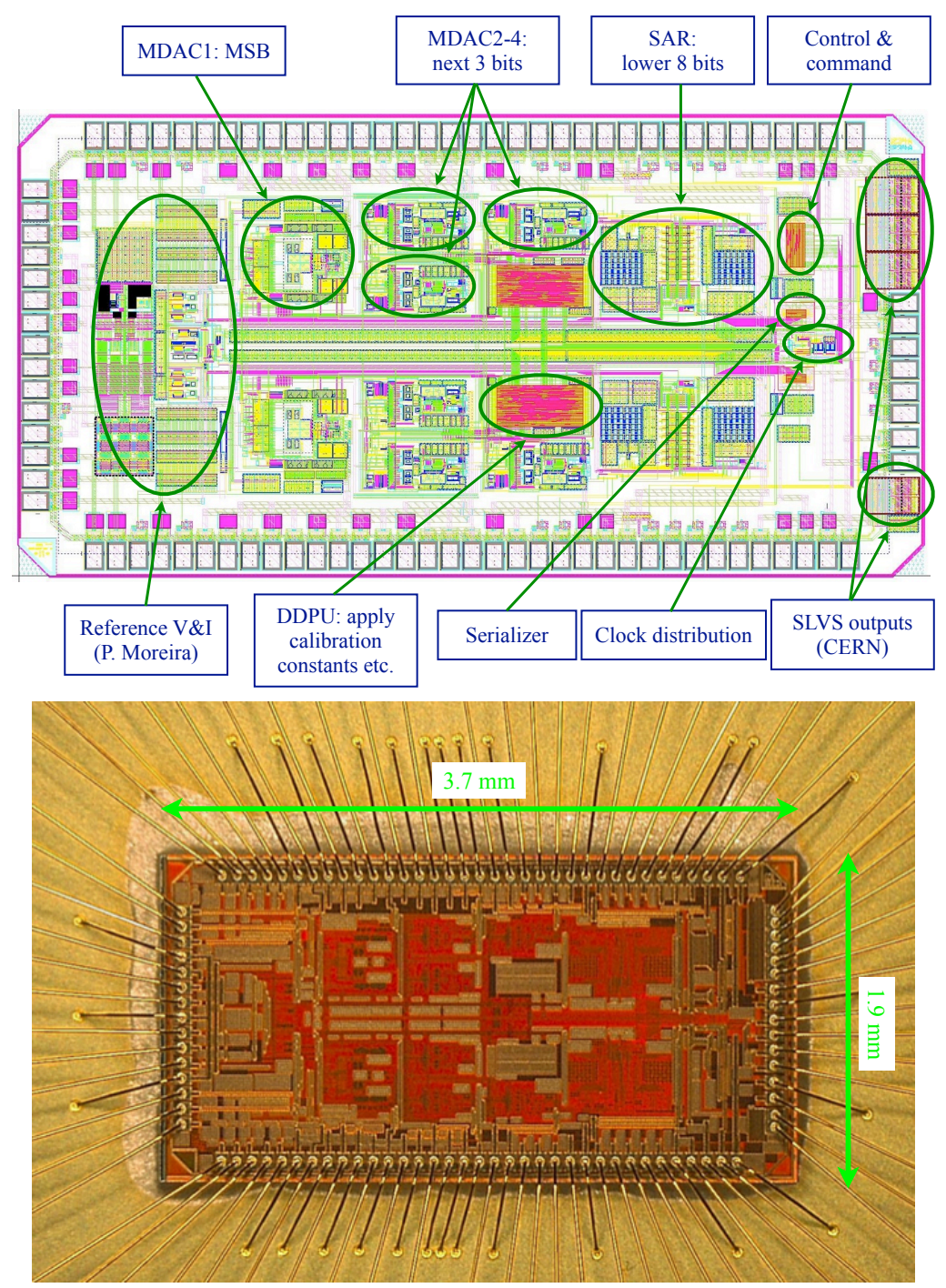

Figure 6: Schematic diagram and photo of the nevis12 chip: a dual channel, 12-bit ADC test chip. 
The Daughter Board preserves the philosophy of redundancy, with each half handling 6 phototubes with a separate Kintex-7 FPGA. The DB handles the high-speed optical communications, which is currently envisioned to occur using radiation tolerant $40 \mathrm{Gbps}$ optical modulators. This communications pipeline will send all tile cell data to the sROD at $40 \mathrm{MHz}$ for recording and formation of a digital trigger. An additional benefit of the optical communication of the trigger (as opposed to the current analog tower sum sent over $70 \mathrm{~m}$ cables) is much lower noise, allowing for better jet trigger turn-on resolution.

The Argonne and Chicago groups are also conducting R\&D on optical communications. At ANL, modifications of a Luxtera modulator-based optical transmitter are being studied and prototypes will be radiation tested. Modulators are faster than commercial vcsel-based transmitters, achieving transmission rates of greater than $10 \mathrm{Gbps}$ with very low error rates and likely high radiation tolerance. Use of a modulator rather than a vcsel transmitter could reduce system cost considerably. The University of Chicago group is setting up a radiation test facility at FNAL to study the single event upset rate in the optical transmitters and FPGA controls. This test facility will simulate the HL-LHC cavern environment.

Currently R\&D is being conducted on three potential front-end amplifier/shaper boards. These boards process the phototube signal and send it to the Main Board, control the calibrations via charge injection and also the calibration achieved using a Cs source (this requires a slow integrator). A redesign of the current 3-in-1 card has been prototyped by the University of Chicago group and has been shown to be radiation tolerant and extremely linear; this card sends LVDS analog data to ADCs on the Main Board.

Two alternative front end boards are also being studied. These are ASIC-based with internal ADCs. One version is a joint effort of the Argonne ATLAS group and the FNAL CMS group to produce a new version of the QIE ASIC. This chip integrates the input current and digitizes in 4 gain ranges. The other ASIC R\&D uses the current conveyor concept to digitize three gain ranges and incorporates a custom radiation tolerant ADC. The decision on which front-end card to be incorporated in Phase-II will only be made after beam testing of all three options.

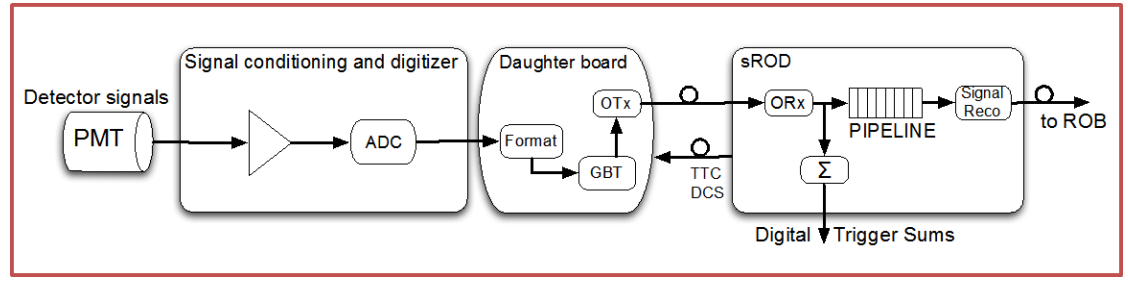

Figure 7: Tile electronics in Phase-II.

\subsection{Off-Detector Electronics}

The main tasks of the off-detector electronics for the calorimeters are the reception of digitized data from the FEBs, data filtering and processing, as well as data transmission to the trigger and DAQ systems. The total input data rate for the LAr off-detector electronics amounts to about $140 \mathrm{Tbps}$.

The central components will be Read-Out Driver boards (ROD) implemented in ATCA standard. These receive the data using serial optical links on multi-fiber ribbons. Conversion to electronic signals is performed by commercial components and deserialization is handled by fast FPGA transceivers. The RODs will apply digital filtering using FPGAs to calculate calibrated energy deposits together with the signal time for each cell as well as signal quality criteria. The digital filtering will correct for electronic and pile-up noise and will be adjustable to the expected high-luminosity conditions by parametrized FPGA algorithms. The RODs will buffer the processed data in digital memory blocks according to 
the number of hardware trigger levels and their corresponding trigger latencies, until data are eventually transmitted to the DAQ system. The data from the individual LAr cells are further processed by the RODs in order to provide input to the Level-1 hardware trigger. The signal processing may go beyond simple sums of cell energy or transverse energies. More complex algorithms like the extraction of features of electromagnetic shower shapes or fast tagging of $\pi^{0} \rightarrow \gamma \gamma$ signals using the finely segmented first calorimeter layer may be possible given the availability of the full granularity of the LAr calorimeters. The detailed algorithmic layout is the subject of on-going $R \& D$, which will take into account how many channels can be concentrated in one processing FPGA and the latency required by the trigger system.

US R\&D activities at Brookhaven National Laboratory, the State University of New York at Stony Brook and the University of Arizona tackle all aspects of this work: use of fast, small form-factor optical transceivers, data handling and processing in high-end FPGAs, and optimal exploitation of the ATCA standard for this application. It should be noted that by going to ATCA, only six racks will be needed to read out the full LAr calorimeter, to be compared with the $17 \mathrm{VME}$ crates currently installed, even though the data volume will have been multiplied by more than 50 .

For the TileCal, the groups at Michigan State University and the University of Texas at Arlington are central to this effort. They are working with engineers at CERN and IFIC Valencia to prototype the system and devise detector control protocols.

\subsection{Summary of Main Calorimeter R\&D Areas}

Areas in which US groups are playing a leading role are listed below.

1. Analog Signal Processing: The liquid argon calorimeter signals need to be amplified and shaped to reduce sensitivity to pile-up and electronics noise. ASIC design efforts based on silicongermanium bipolar technology have shown amplification and shaping can be integrated in a single chip without compromising signal quality.

2. Analog to Digital Conversion: The large number of channels and stringent specifications make ASIC ADC solutions very attractive and cost effective. Integration of additional, ATLAS-specific functionalities will allow to further optimize the readout architecture.

3. High Bandwidth Data Transmission: The ATLAS calorimeters will transmit over 200 Tbps of data off-detector in Phase-II. Low power, radiation tolerant data transmission will be key to realize this on the front-end, while highly integrated solutions (optical links on FPGAs) would be very attractive off-detector. 


\section{Trigger and Data Acquisition}

Running conditions anticipated during Phase-II LHC operations cause significant stresses on the ATLAS Trigger and Data Acquisition (TDAQ) systems. Luminosities of up to $7 \times 10^{34} \mathrm{~cm}^{-2} \mathrm{~s}^{-1}$, leading to an average number of proton-proton collisions per bunch crossing, $\langle\mu\rangle$, of approximately 200 every $25 \mathrm{~ns}$, require major upgrades of the TDAQ in the areas of dataflow and event processing capabilities. Work has already started in evaluating technologies that will allow us to meet these challenges. Many of these solutions are expected to take advantage of advances in commercially available hardware yielding an upgraded system that is more uniform and easily maintainable than the current TDAQ, but that is also flexible enough to adjust to changes in our goals as understanding of the physics driving the upgrades advances.

\subsection{Physics Motivation}

Stresses on the TDAQ system at luminosities expected to reach $7 \times 10^{34} \mathrm{~cm}^{-2} \mathrm{~s}^{-1}$ are one of the main motivations for Phase-II upgrades of the ATLAS experiment. In order to achieve the physics goals outlined in Ref. [1], the TDAQ will have to maintain or improve upon performances achieved in 2012 running and those foreseen to be realized in the ATLAS Phase-I upgrade program. A brief summary of trigger requirements needed to maintain sensitivity to important physics channels is given in Table 1.

Table 1: Trigger requirements to maintain sensitivity to selected physics channels.

\begin{tabular}{ccc}
\hline Channel & Trigger & Target Thresholds \\
\hline$W, Z$ & single-lepton $e, \mu$ & $p_{\mathrm{T}} \sim 20 \mathrm{GeV}$ \\
$t \bar{t}$ & di-jet & $E_{\mathrm{T}} \sim 60-80 \mathrm{GeV}$ \\
$H H \rightarrow b \bar{b} \gamma \gamma$ & di-photon & $E_{\mathrm{T}} \sim 10 \mathrm{GeV}$ \\
VBF & single-lepton + forward jets & $E_{T}^{\text {jet }}>50 \mathrm{GeV}$ \\
SUSY & single-lepton $+E_{T}^{\text {miss }}$ & $p_{T}^{\ell} \sim 20 \mathrm{GeV}, E_{T}^{\text {miss }} \sim 150 \mathrm{GeV}$ \\
\hline
\end{tabular}

All aspects of the ATLAS TDAQ system are impacted by these requirements. However one of the main drivers of the Phase-II TDAQ upgrade is the desire to maintain Level-1 trigger thresholds for isolated electrons and muons at around $20 \mathrm{GeV}$, with an accept rate of $20 \mathrm{kHz}$ or less, for luminosities of up to $7 \times 10^{34} \mathrm{~cm}^{-2} \mathrm{~s}^{-1}$. Estimates of the Level-1 EM trigger rate vs. threshold are shown in Figure 8 (a) for the EM trigger configuration anticipated in Phase-I. Even for isolated EM triggers, a threshold of 40 $\mathrm{GeV}$ or more would be required to achieve an accept rate of $20 \mathrm{kHz}$ or lower. However, Figure 8 (b) shows that raising the Level-1 lepton trigger thresholds from their current values (around $20 \mathrm{GeV}$ ) to the $40 \mathrm{GeV}$ required to meet bandwidth limitations would reduce acceptance by a factor of 1.5-3 depending on the physics channel.

\subsection{TDAQ System before Phase-II}

The trigger architecture currently used by ATLAS and foreseen through the end of Phase-I running consists of three levels.

1. Level-1 (L1) uses custom hardware to construct trigger Regions of Interest (ROIs) based on information from the Calorimeters and Muon system and takes advantage of correlations between objects using a Topological Trigger Processor. During Phase-I running the Level-1 trigger system will generate trigger accepts at an average rate of $100 \mathrm{kHz}$, with a latency of approximately $2.5 \mu \mathrm{s}$. An overview of the L1 system during Phase-I operation is given in Figure 9 (a). 


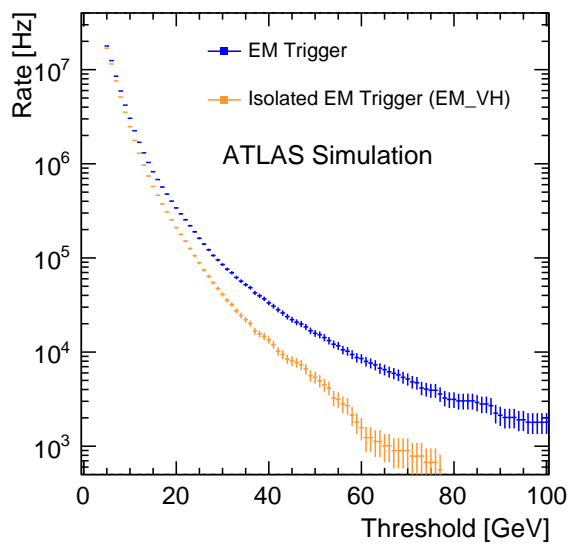

(a)

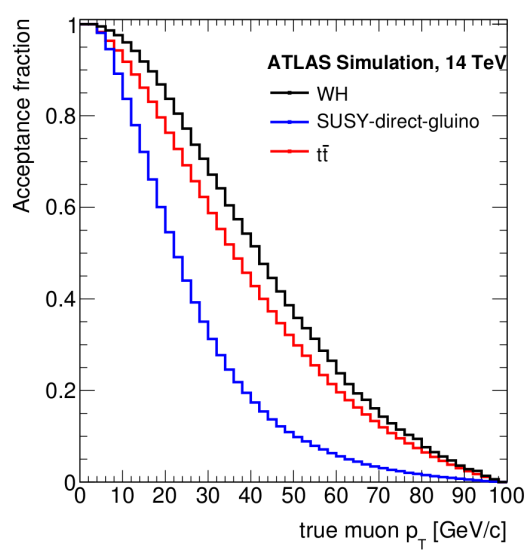

(b)

Figure 8: (a) Projected EM trigger rates for the Phase-I trigger system at $\langle\mu\rangle=115$, corresponding to $4 \times 10^{34} \mathrm{~cm}^{-2} \mathrm{~s}^{-1}$, as a function of trigger threshold. (b) Acceptance of muons from $t \bar{t}, W H$, and SUSY processes as a function of the true muon momentum.

2. Level-2 (L2) is implemented in software on a farm of PCs and constructs triggers using information from all ATLAS sub-systems, but only in ROIs identified by L1.

3. Event Filter (EF) runs on the same farm of PCs as L2, but has access to data from the full event and uses algorithms similar, if not identical, to those run in offline reconstruction. Trigger accepts from the $\mathrm{EF}$ at a rate of up to $1 \mathrm{kHz}$ are foreseen in Phase-I.

L2 and the EF, which in Phase-I will run in the same CPU along with Event Building, are collectively referred to as the High Level Trigger (HLT).

The ATLAS DAQ is centered around system-specific Readout Drivers (RODs) that collect data from the individual sub-system front ends (FE) on L1 accepts, and the common Readout System (ROSs) that provides this data on request to the HLT. The configuration of the DAQ system, as it is foreseen in Phase-I running, is shown in Figure 9 (b).

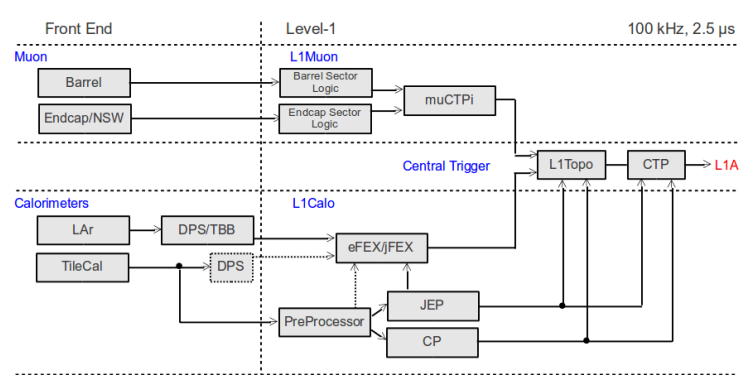

(a)

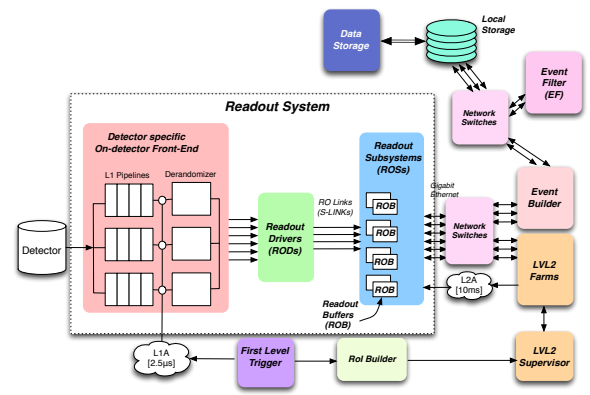

(b)

Figure 9: Functional diagrams of: (a) the L1 trigger system; and (b) the TDAQ in Phase-I. Note that in Phase-I EF, Event Builder, and Level-2 nodes will be implemented in the same processor. 


\subsubsection{Involvement of US Groups in the TDAQ}

The US has played a strong role in the development and operation of the ATLAS TDAQ system. US groups are making major contributions to TDAQ effort in the following areas:

- Calorimeter and Muon sub-system electronics that provide data to the L1 trigger system;

- L1 Calorimeter Trigger upgrades in Phase-0 and Phase-I;

- the L2 Fast Tracker (FTK) trigger;

- the Region-of-Interest Builder;

- development of general-purpose, ATCA-based RODs;

- HLT and DAQ core software, particularly in the areas of dataflow and parallelization.

\subsection{Phase-II Trigger Architecture}

\subsubsection{Rate Estimates for the Trigger at Phase-II Luminosities}

The main issues in continuing to operate the Phase-I trigger system at luminosities up to $7 \times 10^{34} \mathrm{~cm}^{-2} \mathrm{~s}^{-1}$ (a factor of 2-3 higher than those foreseen in Phase-I) come from the L1 system. The Phase-I L1 trigger is constrained to operate with a maximum latency of $2.5 \mu \mathrm{s}$ and a maximum accept rate of $100 \mathrm{kHz}$ due to detector readout capability. This limits possibilities for coping with higher rates expected in Phase-II.

Estimates of the rates of Phase-I L1 leptons triggers have been made based on simulations of the functionality of the Phase-I electromagnetic calorimeter Feature Extractors and extrapolations of the performance of the muon trigger using existing data, including the expected performance of the new forward muon chambers, the New Small Wheels. These estimates indicate that a single electron/photon trigger with an $E_{\mathrm{T}}$ threshold of $25 \mathrm{GeV}$ including hadronic isolation requirements would produce a L1 rate of $125 \mathrm{kHz}$. To achieve a manageable rate of $20 \mathrm{kHz}$ would require raising this threshold to 40 $\mathrm{GeV}$. For single muon triggers with a $p_{\mathrm{T}}$ of $20 \mathrm{GeV}$ rates above $40 \mathrm{kHz}$ are predicted, depending upon assumptions made about background conditions. In both of these cases, ATLAS physics goals would be severely compromised by L1 trigger restrictions. Estimates of expected Phase-I L1 trigger rates for a variety of different triggers at $7 \times 10^{34} \mathrm{~cm}^{-2} \mathrm{~s}^{-1}$ are given in Table $2[2]$.

\subsubsection{Proposed Phase-II Architecture}

The architecture of the Phase-II trigger system is driven by the ATLAS physics goals described in Ref. [1] and by constraints imposed by the detector sub-systems. As mentioned above, one of the most stringent physics requirements is the need to maintain efficient L1 single-lepton triggers with thresholds in the 20 $\mathrm{GeV}$ range. On the detector side, planned Phase-II upgrades relax the L1 rate and latency limitations from their Phase-I values of $100 \mathrm{kHz}$ and $2.5 \mu \mathrm{s}$. However, the inaccessibility of approximately $30 \%$ of the electronics of the MDT system's Barrel Inner (BI) layer results in this system being the new bottleneck. The existing MDT electronics can operate with a latency of up to $20 \mu$ s at a maximum L1 accept rate of $\sim 200 \mathrm{kHz}$.

As can be seen from Table 2, the total rate for L1 triggers that allow ATLAS to achieve its Phase-II physics goals using the Phase-I hardware exceeds the $200 \mathrm{kHz}$ maximum by a factor of 2.5 . The strategy proposed by ATLAS to deal with this involves creating a two-stage hardware trigger. Level-0 (L0), which will use the Phase-I L1 trigger hardware, accepts events at a rate of $500 \mathrm{kHz}$ with a latency of $6 \mu \mathrm{s}$. Following an L0 accept a new L1 system, using additional information and more sophisticated algorithms, will reduce the accept rate to the $200 \mathrm{kHz}$ target with an added latency of $14 \mu \mathrm{s}$. Thus, the 
Table 2: Expected trigger rates at $7 \times 10^{34} \mathrm{~cm}^{-2} \mathrm{~s}^{-1}$ for the L1 Phase-I and the baseline split L0/L1 Phase-II trigger systems. The EM triggers all assume the hadronic energy veto (VH) is used. For the photon and di-photon triggers it is assumed that the full granularity in the L1 calorimeter trigger will bring an additional factor 3 in background rejection power. The $\tau \tau$ trigger rate assumes a factor 2 reduction in the tau fake rate from the eFeX. The exclusive rates for $e \tau$ and $\mu \tau$ are not included as these will depend strongly on the exact trigger menu and trigger thresholds used. The rates for the JET and MET triggers are estimates based on an extrapolation of the current fraction of the trigger budget used for these triggers.

\begin{tabular}{llcc}
\hline Object(s) & \multirow{2}{*}{ Trigger } & \multicolumn{2}{c}{ Estimated Rate } \\
& & Phase-I L1 / Phase-II L0 & Phase-II L1 \\
\hline$e$ & EM20 & $200 \mathrm{kHz}$ & $40 \mathrm{kHz}$ \\
$\gamma$ & EM40 & $20 \mathrm{kHz}$ & $10 \mathrm{kHz}$ \\
$\mu$ & MU20 & $>40 \mathrm{kHz}$ & $10 \mathrm{kHz}$ \\
$\tau$ & TAU50 & $50 \mathrm{kHz}$ & $20 \mathrm{kHz}$ \\
\hline$e e$ & 2EM10 & $40 \mathrm{kHz}$ & $<1 \mathrm{kHz}$ \\
$\gamma \gamma$ & 2EM10 & as above & $\sim 5 \mathrm{kHz}$ \\
$e \mu$ & EM10_MU6 & $30 \mathrm{kHz}$ & $<1 \mathrm{kHz}$ \\
$\mu \mu$ & 2MU10 & $4 \mathrm{kHz}$ & $<1 \mathrm{kHz}$ \\
$\tau \tau$ & 2TAU15I & $40 \mathrm{kHz}$ & $2 \mathrm{kHz}$ \\
Other & JET + MET & $\sim 100 \mathrm{kHz}$ & $\sim 100 \mathrm{kHz}$ \\
\hline Total & & $\sim 500 \mathrm{kHz}$ & $\sim 200 \mathrm{kHz}$ \\
\hline
\end{tabular}

total latency from the L0/L1 system matches the $20 \mu$ s constraint from the MDT readout electronics. Four new features at L1, made possible by changes to the ATLAS detector in Phase-II, are foreseen to make possible this extra rate reduction.

1. Tracking Information: Association of tracks found using data from the silicon inner tracker (ITK) to calorimeter objects and to muons will provide a substantial reduction in the electron, muon, and tau trigger rates.

2. Full Granularity Calorimeter Information: All data from both the LAr and TileCal calorimeters will be available to the L1Calo trigger providing extra background rejection in (di)photon and (di)tau triggers.

3. MDT Information: Muon track momentum reconstruction, using the precise Monitored Drift Tubes (MDT), will be possible at L1. This is particularly effective at rejecting background for relatively low momentum muons.

4. Topology: The Phase-I L1Topo trigger will be enhanced to include new information from L1Track, L1Calo, and L1Muon.

The proposed Phase-II L0/L1 trigger architecture is shown in Figure 10. Its effect on estimated trigger rates is summarized in Table 2. More information on the individual elements of the system is given in the following and in [2].

\subsection{Calorimeter Trigger}

As described in Section 3, the entire calorimeter front-end and back-end electronics systems will be replaced in Phase-II, allowing digitization of all channels on every bunch crossing and transmission of 


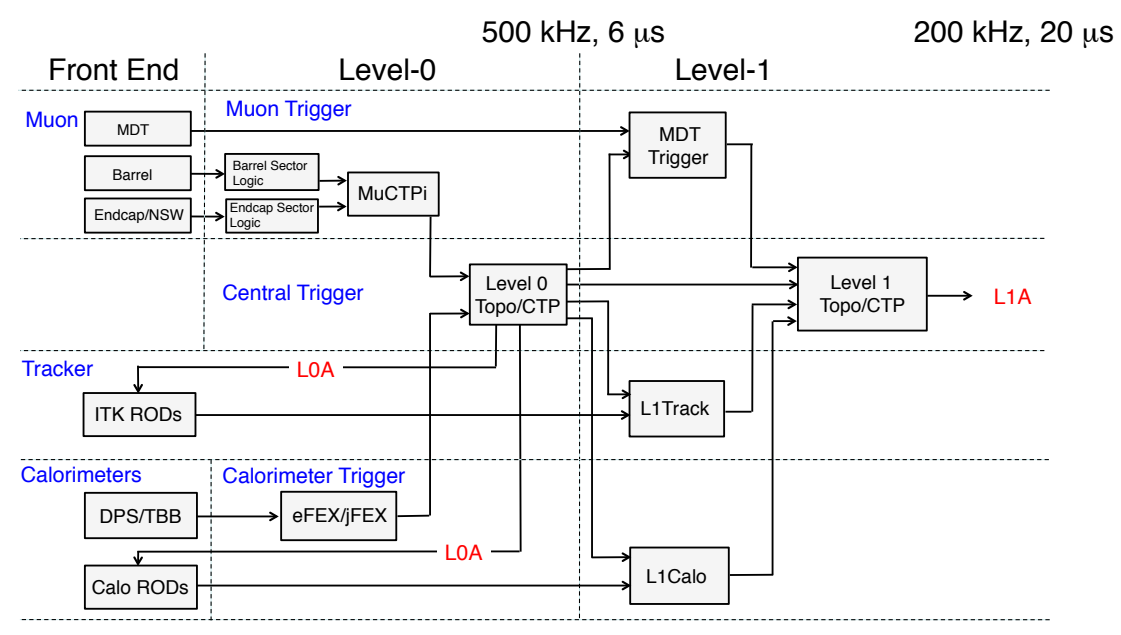

Figure 10: Functional diagram of the L0/L1 trigger system in Phase-II.

this data off-detector. The low-level calorimeter trigger in Phase-II will take advantage of this new, higher-granularity data and will be separated into two pieces. Level-0 (L0Calo) will approximate the functionality of the Phase-I L1Calo but will produce accepts at a much higher rate. Level-1 (L1Calo) will use the full-granularity calorimeter information and more sophisticated algorithms to process events at the average $\mathrm{L} 0$ accept rate of $500 \mathrm{kHz}$, up to a possible peak rate of $20 \mathrm{MHz}$.

The Phase-II L0Calo system will use electron and jet feature extractors (FEXs) as in the Phase-I L1Calo. However, L0Calo inputs will differ from those in Phase-I due to higher precision data being available at the trigger level in the new calorimeter readout scheme. As in Phase-I, HCAL data will be divided into regions of size $0.1 \times 0.1$ in $\eta \times \phi$, while four layers of LAr ECAL data (pre-sampler and three ECAL sampling layers) will be delivered in the "1-4-4-1" arrangement. In this arrangement, the first two ECAL sampling layers have a four times finer segmentation in $\eta(0.025 \times 0.1$ in $\eta \times \phi)$ than the pre-sampler and third ECAL sampling layers. All of this data is sent to the L0Calo on 4064, $10 \mathrm{~Gb} / \mathrm{s}$ optical links, where it will be processed in the FEXs using firmware modified from the Phase-I versions, but running similar algorithms to Phase-I L1Calo.

The Phase-II L1Calo system will have access to full granularity calorimeter data in Regions of Interest (ROIs) defined by L0 and will therefore be able to deliver improved measurements of the energies and positions of trigger objects. For example, the second ECAL sampling layer has a full granularity segmentation of $0.025 \times 0.025$ in $\eta \times \phi$ (compared to $0.025 \times 0.1$ at L0Calo), while the very finely grained first ECAL sampling layer $(0.003125 \times 0.1$ in $\eta \times \phi)$ could be used to suppress EM trigger backgrounds due to $\pi^{0} \rightarrow \gamma \gamma$ decays.

A common area of R\&D in both of these systems centers on the large data volumes ( 2 Tbps in L0Calo and 200 Tbps in L1Calo) sent to and among system elements.

\subsection{Muon Trigger}

Upgrades to the Phase-I L1Muon system required to retain $p_{\mathrm{T}}$ thresholds in the $20 \mathrm{GeV}$ range for the split L0/L1 trigger scheme in Phase-II fall into three main categories. First, most muon readout electronics in the barrel and endcaps will need to be replaced to deal with the new trigger architecture. Second, tracking performance of the Resistive Plate Chambers (RPCs) in the barrel can be improved by using time-overthreshold information. Finally, precise hit information from the Monitored Drift Tubes (MDTs) will be 
added to the L0 or L1 trigger logic to improve muon trigger momentum resolution, aiding in the rejection of low-momentum backgrounds.

For the MDTs, bunch-crossing identification for hits in individual tubes will be transmitted to the L0 or L1 trigger systems using high speed optical links. This information will allow $p_{\mathrm{T}}$ reconstruction at L0 or L1 with a quality similar to that of the current L2 muons, resulting in a reduction of the L1Muon rate by a factor of approximately three over much of the detector.

The L1Muon $p_{\mathrm{T}}$ resolution can also be sharpened by using the charge distribution in clusters of RPC $\eta$ strips. This charge distribution can be accessed because the signal duration in the current RPC frontend electronics is correlated to the input charge. Measurements with a TDC could thus deliver the charge distribution in adjacent strips, from which a centroid could be formed. R\&D is currently under way to evaluate the potential of this idea in detail.

\subsection{Level-1 Track Trigger}

Despite improvements to L1Calo and L1Muon in Phase-II, these systems alone are unlikely to entirely meet the physics goals of ATLAS. For this reason a crucial element of the ATLAS Phase-II upgrade program is the addition of a new trigger system at L1 using information from the Inner Detector. Charged particle tracks reconstructed by this L1Track trigger would be combined with EM objects from L1Calo and muon candidates from L1Muon to yield significant rate reductions. Current estimates, using simulation studies and extrapolations of current L1 trigger rates from data, indicate that reductions in the rate of more than a factor of five compared to $\mathrm{L} 0$ for $20 \mathrm{GeV}$ single muon triggers and $18 \mathrm{GeV}$ isolated single electron triggers using L1Track information are possible. Examples of these studies are give in Figure 11.

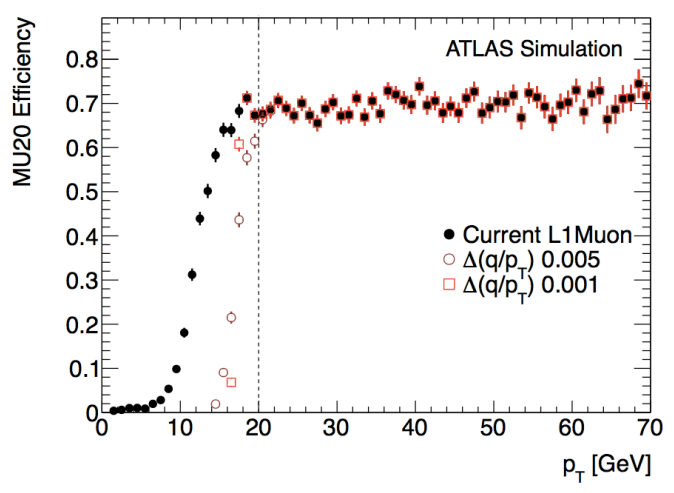

(a)

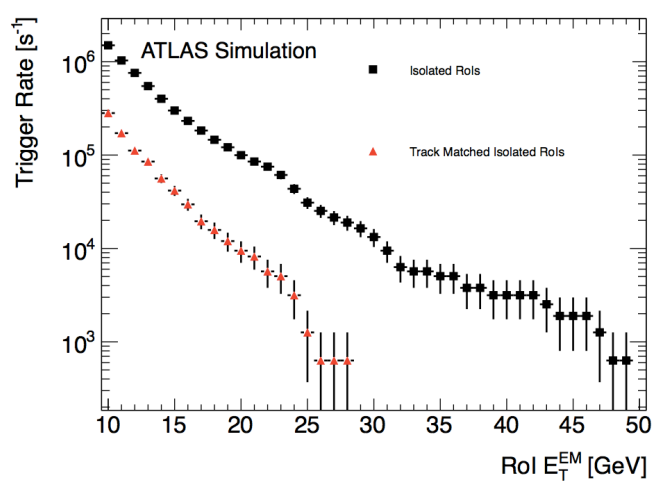

(b)

Figure 11: (a) Muon trigger efficiencies with a $20 \mathrm{GeV}$ threshold as a function of true muon $p_{\mathrm{T}}$ after matching to a true muon, assuming a track trigger with different $p_{\mathrm{T}}$ resolutions. (b) Trigger rate vs L1 EM cluster $E_{\mathrm{T}}$ threshold for simulated minimum bias events with $\langle\mu\rangle=70$.

Two possible L1Track architectures are currently under study. The baseline architecture, RoI-Driven L1Track, would provide all tracks in areas near L0 EM and Muon RoIs, while an alternate, Self-Seeded L1Track, would reconstruct all high momentum $\left(p_{\mathrm{T}}>10 \mathrm{GeV}\right)$ tracks independent of L0 RoIs.

In the RoI-Driven approach, Inner Detector data, buffered in on-detector electronics, is read out through associated Readout Drivers (RODs) only for those regions near a L0 EM or Muon RoI. This regional readout feature is already included in the design of Phase-II front-end readout ASICs for the strip tracker upgrade. The main challenge will be to perform the data readout and tracking within the 
Phase-II L1 latency budget of $20 \mu$ s. Preliminary studies indicate that this will be feasible.

The alternate, Self-Seeded, approach to L1Track requires a huge reduction in inner detector data volume. This could be accomplished by a combination of the use of fewer tracking layers in the trigger, and the early rejection of low $p_{\mathrm{T}}$ tracks using cluster sizes and the inclination angle between the hits in stacked double strip layers. This approach requires major changes to the layout of the inner detector layers over what is currently assumed (see Section 2).

In both approaches L1Track pattern recognition might be done using associative memory (AM) technology similar to that being developed for the Phase-I ATLAS L2 Fast Tracker (FTK) [3]. R\&D is ongoing to exploit novel ASIC design technologies, such as 3-D, to meet the experiment's goals here.

\subsection{Central Trigger System}

Changes to the Central Trigger (CT) for Phase-II include development of separate L0 and L1 CTs, with topological capabilities included in each; and an update of the trigger, timing, and control (TTC) system. As can be seen from Figure 10, the L0CT and L1CT are structurally rather similar. The intent is to use the same technologies for these systems to ease design and maintenance requirements. For the upgraded TTC, the required topology matches that of a Passive Optical Network (PON). Studies are under way to assess the feasibility of using commercially available components for the final system.

\subsection{High Level Trigger}

In order to remain within limitations of $5-10 \mathrm{kHz}$ on the total data recording rate, the Phase-II High Level Trigger (HLT) will need to provide a rejection factor of 20-40 beyond the L1 accept rate of 200 kHz. To achieve this goal, the HLT will employ offline-type selections and will rely increasingly on multi-object signatures. Advances in computer hardware performance over the next 10 years will help here, but upgrades to the HLT farm as well as improvements to selection software will also be required. One particularly promising area of development in HLT software involves the increased use of many-core architectures (e.g. GPUs) and parallelization of code. This will require significant changes to both the HLT framework and to the algorithms themselves. Another challenge will be to maintain commonality with offline software, a feature that substantially eases the burden of code development and maintenance.

\subsection{Data Acquisition}

Increased L1 accept rates $(200 \mathrm{kHz})$ and larger event sizes $(>4 \mathrm{MB})$ due to high levels of pileup at Phase-II luminosities indicate that at least a factor of four increase in bandwidth will be required of the ATLAS data acquisition system in Phase-II beyond that needed in Phase-I running. Additionally, higher values of pileup $\left(\langle\mu\rangle\right.$ approaching 200 at luminosities of $\left.7 \times 10^{34} \mathrm{~cm}^{-2} \mathrm{~s}^{-1}\right)$ imply a significant increase in event processing time. Meeting these challenges will require changes in both hardware and software. Principal areas under study are: network technologies, online databases, information sharing mechanisms, and expert systems. Although it is premature to specify details of an upgraded data acquisition system, given the speed at which the relevant technologies are developing, a possible architecture for Phase-II readout is given in Figure 12. Figure 9 (b) shows the current readout architecture for comparison. The Phase-II system aims for increased levels of commonality across detector sub-systems, taking advantage of emerging technologies and commercially available components. For example, aggregators feeding commercial, high-speed network switches allow data from different sub-systems to be read out into a common Readout Driver (ROD). Aside from simplifying issues of production and maintenance, this scheme could allow the possibility of re-organizing readout connectivity without physical re-cabling as well as leading to increased flexibility in terms of scalability and staging. 


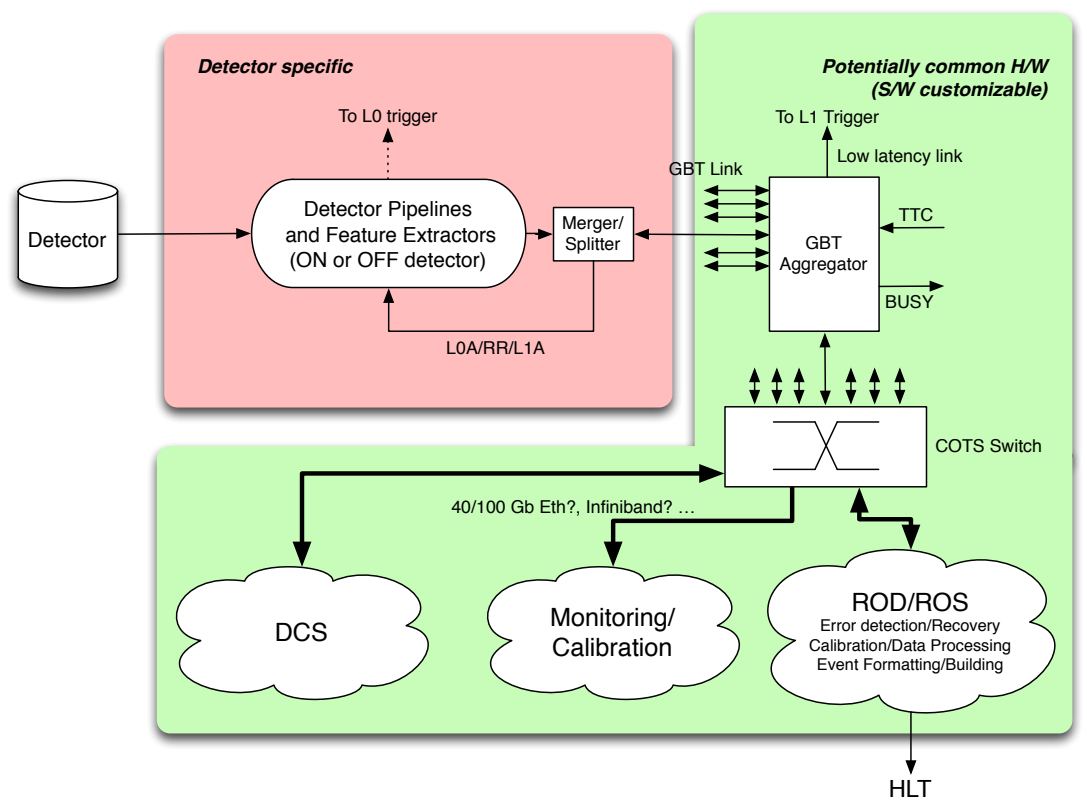

Figure 12: Readout architecture overview for the Phase-II upgrade.

\subsection{Summary of Main TDAQ R\&D Areas}

Aside from system-specific studies, the main areas of TDAQ-related R\&D that are of general interest are summarized below. Work in most of these areas is just starting. However, most of the approximately 15 US institutes currently participating in TDAQ activities are playing strong roles in upgrade R\&D planning.

1. High-speed optical links: Particular areas of concentration here are in the Calorimeter trigger system where approximately 200 Tbits of data must be transmitted per second in L1Calo; and in detector front-end to back-end links, which must operate at speeds of up to $10 \mathrm{Gbps}$ in high radiation environments.

2. Novel ASIC technologies: For example, 3D technologies for use in Track Trigger Associative Memory chips.

3. ATCA applications: Standardized RODs implemented as ATCA cards could save costs by providing high performance, flexible platforms for a wide variety of readout applications.

4. Parallelism and multi-core processing applications: $R \& D$ is ongoing in using multi-threaded code and GPU architectures to improve performance and open new possibilities in the HLT and DAQ.

5. High-speed switching networks: Clever use of commercial high-speed network switches within a common readout framework could provide a flexible, cost-effective solution to sub-system readout needs. 


\section{Conclusion}

The ATLAS Phase-II upgrade will allow exploration of the Energy Frontier to the highest masses as well as precision studies of the Higgs boson and any other new phenomena, fulfilling the promise of the LHC. To achieve this requires significant upgrades to the detector focused primarily on a new tracking detector, new calorimeter electronics, and a new TDAQ system. All of these upgrades to ATLAS require nearterm R\&D on sensors, electronics, and high bandwidth data-transmission components, areas in which US groups are already playing leading roles. Upgrades are also planned to the ATLAS muon system, particularly to the readout electronics and trigger systems. US groups have broad expertise in these areas and could contribute effectively to the Phase-II muon system upgrade effort were more R\&D money to become available. Although the focus of this whitepaper has been on ATLAS and the LHC, many of the advances planned will be of general value to the community as they require mastering the latest technologies and exploring their limits. 


\section{References}

[1] Tony Liss and Jason Nielsen. Snowmass Whitepaper: ATLAS Physics Sensitivity. in preparation, 2013.

[2] Philip Allport and Marzio Nessi. Letter of Intent for the Phase-II Upgrade of the ATLAS Experiment. CERN-LHCC-2012-022 https://cds. cern.ch/record/1502664/, 2012.

[3] Philip Allport and Marzio Nessi. Letter of Intent for the Phase-I Upgrade of the ATLAS Experiment. CERN-LHCC-2011-012 https://cdsweb. cern.ch/record/1402470/, 2011.

[4] N Dressnandt, F M Newcomer, S Rescia, and E Vernon. LAPAS: A SiGe Front End Prototype for the Upgraded ATLAS LAr Calorimeter. ATL-LARG-PROC-2009-017, 2009. 


\section{Instrumentation for the Energy Frontier}

August 4, 2013

\section{Contents}

1 Introduction $\quad 1$

2 Physics at the energy frontier $\quad 2$

3 Benchmark processes $\quad 3$

4 Challenges for energy frontier machines $\quad 4$

4.1 Hadron colliders . . . . . . . . . . . . . . . . . . . . . . . 4

$4.2 \mathrm{e}^{+} \mathrm{e}-$ colliders . . . . . . . . . . . . . . . . . . . 5

$4.3 \mu^{+} \mu^{-}$colliders . . . . . . . . . . . . . . . . . . . . . 6

4.4 Performance goals . . . . . . . . . . . . . . . . . . . . . . . 6

5 Technology R\&D themes $\quad 6$

5.1 Advanced triggers and data acquisition . . . . . . . . . . . . . . 7

5.2 Next generation trackers . . . . . . . . . . . . . . . . . . . . 8

5.3 High resolution hadron calorimeters . . . . . . . . . . . . . . . . . . . 10

5.4 High-rate muon systems . . . . . . . . . . . . . . . . . . . 11

6 Conclusion $\quad 11$

\section{Introduction}

During the last two or three decades, we have explored physics at the energy frontier in ever more challenging environments. First at SLC and LEP, then at the Tevatron, and now at the LHC, we have carried out precision measurements of the parameters of the standard model, we have observed the top quark, and most recently, the Higgs boson. These successes have been made possible by advances in technology and instrumentation that allowed us to build ever more complex detectors. In order to continue our success at the energy frontier further advances in technology will be required. To succeed we need to make technical and scientific innovation a priority as a field. 


\section{Physics at the energy frontier}

The standard model provides us with a seemingly complete description of elementary particle physics as we observe it in the laboratory. Yet we know that it is not a complete theory of the physics of our universe. We know that most of the matter in the universe cannot be explained by the standard model. We know that there are forces in the universe that the standard model does not describe. The energy scale of gravity appears to be so fundamentally different from the energy scales at which we have verified the validity of the standard model that it seems unlikely the standard model could be correct over the entire spectrum of energies that determine the physics of our universe. The recent discovery of the Higgs boson has confirmed the last untested prediction of the standard model. Yet the relatively small mass of the Higgs boson is the reason that we believe the standard model cannot be valid to much higher energy scales than we can probe in the laboratory at this time. We believe that there must be physical phenomena beyond the standard model that should manifest themselves at energies not to much above the energies we currently probe. The big question that we want to illuminate is: what is the nature of this new physics?

Our exploration of particle physics has been characterized by a two pronged approach. On the one hand we have pushed to ever higher energies in order to experimentally probe the structure of the standard model by directly producing particles that cannot be produced at lower energies, such as the top quark and the Higgs boson. On the other hand we have carried out extremely precise measurements of phenomena at lower energies to extract information about the underlying physics. In the cases of both the top quark and the Higgs boson, we had a very good idea at what mass we should expect to find them, based on precision measurements at lower energies. It is the synergy between these two approaches that gives us the confidence that we understand the underlying physics. It is therefore essential that we explore the boundaries of the standard model in both ways. We need to directly look for new massive particles at the energy frontier and dark matter particles at the cosmic frontier as well as carry out ever more precise measurements of lower energy phenomena at the intensity frontier in order to deepen our understanding of the physics that drives our universe. The questions that can be explored at the energy frontier in the coming decades also reflect this two-fold approach.

One of the most important questions is whether the new $125 \mathrm{GeV}$ boson is the minimal Higgs boson of the standard model or part of an extended Higgs sector. In order to find out, we need to measure the properties of the Higgs boson to the best possible precision. This includes measuring the cross sections of its production processes and its branching fractions to determine the complete set of couplings and self-couplings of the Higgs boson. But in addition we also want to search for other manifestations of a potential extended Higgs sector and we want to study WW scattering at high energies to understand what regularizes its amplitude.

In addition, we want to search for manifestations of the new physics that we believe to be there at the $\mathrm{TeV}$ scale. We want to continue our search for superpartners and explore corners of SUSY parameter space that we have not been able to reach. Light stop squarks or compressed spectra could make it very difficult to observe the superpartners that are in our range because their decay products are relatively soft. Of course we also want to search for new massive particles at as high a mass as we can reach. 
In order to maximize our sensitivity to these we want to push beam energies as high as possible. The study of the Higgs boson and the search for low scale SUSY ensure that, even as the beam energy increases, we are still looking at electroweak scale phenomena involving $\mathrm{W}$ and $\mathrm{Z}$ bosons and their decay products. The challenge for our detectors will be to maintain acceptance to relatively soft particles, to maintain large angular acceptance to minimize theoretical uncertainties and retain sensitivity to distinguish between different models should we find something new. Superior spatial and time resolution will be essential for pattern recognition in high occupancy environments.

\section{Benchmark processes}

Here we identify specific benchmark processes that define the detector performance requirements.

Precise measurements of Higgs boson decays define important benchmarks for detector performance. At hadron colliders, $\mathrm{H} \rightarrow \gamma \gamma$ decays require the ability to trigger on photon pairs with low momentum thresholds. In order to achieve optimal diphoton mass resolution the identification of the hard scattering vertex is important $\mathrm{H} \rightarrow \mathrm{ZZ} \rightarrow 4 \ell$ and $\mathrm{H} \rightarrow \mathrm{WW} \rightarrow \ell \nu \ell \nu$ decays require the ability to trigger on multiple leptons with low momentum thresholds. Measuring the branching fractions for $\mathrm{H} \rightarrow \mathrm{b} \overline{\mathrm{b}}$ and especially $\mathrm{H} \rightarrow \mathrm{c} \overline{\mathrm{c}}$ drives the flavor tagging performance of vertex detectors. The latter can probably only be measured at lepton colliders and $\mathrm{R} \& \mathrm{D}$ is still needed to develop the technology for a vertex detector that achieves the required impact parameter precision of $5 \mu \mathrm{m}$ at high $p_{T}$. The momentum resolution of the tracking system at $\mathrm{e}^{+} \mathrm{e}^{-}$colliders should be $\sigma(1 / p) \approx 2.5 \times 10^{-5}$. This is driven by the Higgs mass measurement using the $\mathrm{Z}$ boson recoil.Separating $\mathrm{H} \rightarrow \mathrm{WW}$ and $\mathrm{H} \rightarrow$ ZZ decays followed by hadronic decays of the vector bosons drives requirements for the jet energy resolution. At hadron colliders the measurement of Higgs boson production by the vector-boson fusion mechanism requires forward coverage for jets up to $|\eta| \approx 4$, including the capability to trigger. Pileup mitigation will be required to be able to veto jets between the two forward/backward jets. The detection of the decay $\mathrm{H} \rightarrow \mu \mu$ drives the detector acceptance and efficiency to detect muons.

Searches for physics beyond the standard model also define some benchmarks. Limits on SUSY models are tightening based on the analysis of the LHC data. However there are corners in parameter space for low-mass SUSY that remain. If the mass spectrum of superpartners is compressed the decay products of superpartners could be rather soft, resulting in relatively low missing transverse momentum and low momentum charged particles. This affects the designs of the trigger and the tracking detector. Triggering on moderate missing $p_{T}$ requires effective pileup mitigation, possibly using tracking at the first trigger level to compute missing $p_{T}$. Large acceptance for leptons and the ability to include low $p_{T}$ trigger terms for leptons in more complex trigger conditions will be important. Calorimeter timing could also help mitigate pileup by separating soft particles from the hard scattering vertex and from pileup interactions.

The range of phenomena for more exotic physics models is wide. The capability to flavor tag very high mometum jets (for example from high mass exotic resonances) could be important. For jet $p_{T}$ values in excess of $500 \mathrm{GeV}$, the b-decay length becomes comparable 
to the radius of the inner layer of vertex detectors. Tracks from such secondary vertices will not leave hits in the innermost vertex detector planes and they could be boosted such that they are very close together in space requiring enahnced two-track separation capabilities. Calorimeter mass resolution could be important for separating closely spaced resonances that decay to jets.

One might ask whether there are any advances in technology that could revolutionize the physics capabilities of particle physics detectors. The omnipresent constraint on the technical capability of a detector is often cost rather than technology limitations. Therefore develoments of techniques that reduce the cost of the large scale detectors required for high energy experiments by an order of magnitude may be the advances that could have the most profound impact on the high energy physics program in the US. An example is forward tracking for the phase 2 LHC detectors. The technology to build trackers that can measure particles with $|\eta|<4$ exists. But constructing the detectors is costly because of the high density of connections that need to be made to sufficienctly pixelated detectors. A technique that would reduce the cost of such detectors by an order of magnitude would make a big impact.

A transformational capability for hadron collider detectors would be the ablility to read out the entire detector for every beam crossing and process the data for the first level trigger decision. Much R\&D goes into the direction of making more and better data available for the lowest level trigger, such as the efforts to develop a level 1 track trigger. Crucial elements that support this trend are the development of low-power, high-bandwidth data links and highly interconnected architectures for the trigger processors.

Besides cost one should also keep an open mind for new ideas from other fields. Examples are the use of novel materials such as graphene or metamaterials, advanced photonics techniques, developments in the telecom industry.

\section{Challenges for energy frontier machines}

Experiments at the energy frontier are dominated by large accelerators and multipurpose collider detectors. We want these detectors to do everything: measure position to microns, measure timing to picoseconds, measure energy deposits to eV using highly pixelated trackers and calorimeters and read out all the data for as many events as possible. All this at low cost, using minimal power, and with low mass. Real detector designs will of course be a compromise between the constraints of the accelerator environment, the physics needs, our technical capabilities, and the available funds.

\subsection{Hadron colliders}

Table 1 lists some parameters for current and possible future hadron colliders [1].

The challenges that face experiments at the LHC and other hadron colliders are high interaction rate, large number of interactions in the same beam crossing, and high radiation doses. The high interaction rate will require an increased rejection power of the trigger system and low power-high bandwidth data links to transport the data out of the detector. Dealing with high pileup requires increased pixelization of the detector and 10-100 ps timing 
Table 1: Hadron collider facilities.

\begin{tabular}{lcccc}
\hline \hline & LHC & HL-LHC & HE-LHC & VLHC \\
\hline time scale & $2015-2021$ & $2023-2030$ & $>2035$ & $>2035$ \\
center of mass energy $(\mathrm{TeV})$ & 14 & 14 & $26-33$ & 100 \\
luminosity $\left(/ \mathrm{cm}^{2} / \mathrm{s}\right)$ & $10^{34}$ & $5 \times 10^{34}$ & $2 \times 10^{34}$ & \\
integrated luminosity $(/ \mathrm{fb})$ & 300 & 3000 & 3000 & 1000 \\
number interactions/crossing & $\approx 50$ & $\approx 140$ & $\approx 225$ & $\approx 40$ \\
bunch spacing $(\mathrm{ns})$ & 25 & 25 & 50 & 19 \\
radiation dose $(\mathrm{Gy} @ \mathrm{R}=5 \mathrm{~cm})$ & $3 \times 10^{4}$ & $5 \times 10^{6}$ & & \\
\hline \hline
\end{tabular}

to separate the particles that emanate from different interactions in the same beam crossing. The increased radiation dose requires the development of radiation hard detector technologies and detector operation at increasingly lower temperatures.

\section{$4.2 \mathrm{e}^{+} \mathrm{e}-$ colliders}

Table 2 lists some parameters for $\mathrm{e}^{+} \mathrm{e}-$ colliders.

Table 2: $\mathrm{e}^{+} \mathrm{e}^{-}$collider facilities.

\begin{tabular}{lccc}
\hline \hline & ILC & CLIC & TLEP \\
\hline timescale & 2028 & $>2035$ & $>2035$ \\
center of mass energy $(\mathrm{GeV})$ & $91-1000$ & $350-3000$ & $91-350$ \\
luminosity $\left(/ \mathrm{cm}^{2} / \mathrm{s}\right)$ & $5 \times 10^{34}$ & $6 \times 10^{34}$ & $56 \times 10^{34}$ \\
integrated luminosity $(/ \mathrm{fb})$ & 1000 & 3000 & 2500 \\
bunch spacing $(\mathrm{ns})$ & 366 & 0.5 & \\
bunches/train & 2625 & 312 & 4400 \\
length of bunch train $(\mathrm{ms})$ & 1 & 0.156 & $\mathrm{n} / \mathrm{a}$ \\
interval between trains $(\mathrm{ms})$ & 199 & 20 & $\mathrm{n} / \mathrm{a}$ \\
collision rate $(\mathrm{Hz})$ & 5 & $<50$ & \\
\hline \hline
\end{tabular}

Challenges at $\mathrm{e}^{+} \mathrm{e}^{-}$colliders are different from hadron colliders. Exploiting the physics potential mandates detectors that have higher granularity and lower mass by one to two orders of magnitude than the current LHC detectors. On the other hand requirements imposed by beam structure, data rate, and radiation dose are much more modest than at LHC[2].

An exception to this is the time structure of the beam at CLIC which foresees bunch trains at $20 \mathrm{~ms}$ intervals. Each train comprises 312 bunches separated by $0.5 \mathrm{~ns}$. There will be at most one $\mathrm{e}^{+} \mathrm{e}^{-}$interaction per train. However, the high CLIC energies and small intense beams lead to significant beam-induced background. Triggerless readout of zero-suppressed data at the end of the bunch train is planned. Hit time resolution of $1 \mathrm{~ns}$ combined with fine detector granularity are essential for efficient suppression of beam-induced background in the data[3]. 


\section{$4.3 \quad \mu^{+} \mu^{-}$colliders}

The vision for a muon collider includes a number of incremental steps. The first step would be a Higgs factory muon collider running at a center of mass energy equal to the Higgs mass and a luminosity of about $10^{31}-10^{32} / \mathrm{cm}^{2} / \mathrm{s}$. The beam energy spread at a muon colider is small enough that it can produce Higgs bosons in the s-channel. This could be followed by an energy frontier accelerator with a center of mass energy of 3-6 TeV and a luminosity of about $10^{34}-10^{35} / \mathrm{cm}^{2} / \mathrm{s}$.

At a muon collider the bunch spacing would be relatively large, $500 \mathrm{~ns}$ for a Higgs factory and $10 \mu \mathrm{s}$ for a high energy collider, leaving lots of time to read out the detector. However, backgrounds are large. For two beams at $0.75 \mathrm{TeV}$ there will be $1.3 \times 10^{10} / \mathrm{m} / \mathrm{s}$ muon decays. The radiation dose will be $10^{3}-10^{4}$ Gy/year. Thus, detectors must be radiation hard. Precision time resolution will be crucial to separate signals from the background and the primary interaction as most of the background is soft and out of time[4,5].

\subsection{Performance goals}

Table 3 summarizes the performance figures used for the "snowmass detector" that served as a reference for hadron collider simulations and corresponds to the typical performance of ATLAS and CMS. Table 4 summarizes the performance goals for lepton colliders.

\begin{tabular}{|c|c|}
\hline performance figure of merit & goal \\
\hline $\begin{array}{l}\text { tracking/muon system acceptance } \\
\text { calorimeter acceptance }\end{array}$ & $\begin{array}{c}|\eta|<2.5 \\
|\eta|<5\end{array}$ \\
\hline $\begin{array}{l}\text { EM energy resolution (central) } \sigma_{E} / E \\
\text { jet energy resolution (central) } \sigma_{E} / E \\
\text { track momentum resolution }\end{array}$ & $\begin{array}{l}8 \% / \sqrt{E} \oplus 0.5 \% \\
60 \% / \sqrt{E} \oplus 3 \% \\
1.5 \% @ 100 \mathrm{GeV}\end{array}$ \\
\hline
\end{tabular}

Table 4: Performance goals for $\mathrm{e}^{+} \mathrm{e}^{-}$collider detectors $[2,3]$.

\begin{tabular}{lc}
\hline \hline performance figure of merit & goal \\
\hline track impact parameter resolution $\sigma_{b}$ & $5 \oplus 10 / p \beta \sin ^{3 / 2} \theta$ \\
track momentum resolution $\sigma_{p T} / p_{T}^{2}$ & $2 \times 10^{-5} / \mathrm{GeV}$ \\
jet energy resolution $\sigma_{E} / E$ & $3.5 \% @ 100 \mathrm{GeV}$ \\
lepton acceptance & $|\theta|>10 \mathrm{mrad}$ \\
time resolution & $1-10 \mathrm{~ns}$ for CLIC \\
\hline \hline
\end{tabular}

\section{Technology R\&D themes}

Instrumentation R\&D will have to address the challenges posed by physics and accelerator environment outlined in the two previous sections. Several themes emerge from these. 
- Pixelization: Continued microelectronics feature size reduction, 3D electronics technologies, and new interconnect technologies will allow finer spatial segmentation of detectors. Low power-high bandwidth links need to be developed for transmission of the increased data volume.

- Timing: Faster sensor technologies and low power, fast electronics are needed to enable precise timing measurements for detector signals. Pixelization and 3D technologies will help create the lower capacitance detectors needed and waveform digitization can provide precise timing information.

- Resolution: Increased pixelization of trackers and calorimeters will improve the resolution of tracking and calorimetry. Imaging calorimeters combined with better understanding of hadron showers could enable a transformational improvement in the resolution of jet energy measurements.

- Mechanics and power: New materials and techniques need to be developed to provide mechanical support for thinner, more highly segmented detectors, reduce the power dissipated in increasing numbers of electronics channels, and improve cooling to enable detectors to run at lower temperature. These could include carbon fiber supports, foamed thermally conductive materials, $\mathrm{CO}_{2}$ cooling, and power delivery using DCDC conversion or serial powering.

- Data transmission: Low power optical interconnects, wireless data transmission, and low power signaling could revolutionize the transmission of data out of the detector.

- Cost: The new technologies must also be more cost effective. Large area arrays utilizing new technologies (as used by LAPPD), wafer scale integration, high yield assembly techniques with active edge sensors could help achieve this goal.

Following we identify three detector areas, trigger and data acquisition, tracking, and hadron calorimetry. These represent the components of the detectors at energy frontier facilities for which new technologies would have the biggest impact. We associate the above R\&D themes with these detector areas and summarize the status of R\&D, based on the white papers submitted to the intensity frontier working group. The full list of white papers can be found at https://www-public.slac.stanford.edu/snowmass2013/Index. aspx.

\subsection{Advanced triggers and data acquisition}

As the instantaneous luminosity at the LHC increases by an order of magnitude, increased pileup leads to non-linear increases in trigger rates. Improved rejection power is needed at each trigger level. Access to the full granularity of the detector at level 1, and a level 1 track trigger provide the data for advanced pattern recognition. Compact high-density optical connectors and low power-high bandwidth optical links[6] help transfer this data out of the detector and ATCA and $\mu$ TCA crate technology with high-speed star and mesh backplanes used by the telecommunications industry provide increased interconnectivity between processors[7]. Processing will benefit from 3D technology for associative memory ASICs and exploit state of the art FPGAs and processing units such as GPUs[8]. 
There are two approaches to designing a L1 track trigger for LHC. A region-of-interest trigger[9] would read out only hits near level 1 electron or muon candidates and help in sharpening $p_{T}$ turnon curves. This will allow raising the $p_{T}$ threshold at level 1 and thereby reduce the rate without loss of efficiency for high $p_{T}$ leptons. The other approach is a selfseeded trigger[10]. This requires on-detector data reduction which can be achieved by using coplanar strip or pixel sensors, closely spaced in radial direction to reject hits from low $p_{T}$ tracks. A self-seeded track trigger could potentially reconstruct all tracks above a moderate $p_{T}$ threshold such as $2 \mathrm{GeV}$ and associate them with primary interaction vertices. This would enable track-based isolation using only tracks from the same vertex thereby mitigating the effect of pileup.

Application specific integrated circuits (ASICs) are fundamental component of instrumentation for all frontiers[11]. They allow high channel density, improve analog performance (e.g. noise, speed), enable data reduction, lower power dissipation, and reduce cabling and mass. R\&D is needed to develop high-speed waveform sampling[12], pico-second timing, lownoise high-dynamic-range amplification and shaping, digitization and digital data processing, high-rate data transmission, low temperature operation, and radiation tolerance.

\subsection{Next generation trackers}

Trackers need to provide excellent momentum and impact parameter resolution in high rate environments. This requires improved two track separation. The ability to assign a time stamp to hits will be important to reduce backgrounds especially at CLIC and at muon colliders. Thinner sensors are required for the short charge collection times that enable precise timing measurements. Spatial resolution also is ultimately limited by sensor thickness. Charges spread less in thinner sensors providing better spatial resolution. Thinner sensors also reduce mass, leakage current, and power dissipation. At hadron colliders and muon colliders, radiation hardness is a must for tracker sensors. To mitigate the effects of radiation damage, sensors must be operated at low temperatures. On the other hand, power distribution for the increased channel count and fast data links is an issue. These trackers will require the development of multipurpose structures for mechanical support and more efficient cooling.

Hybrid pixel detectors are the current state-of-the-art for pixel trackers[13]. They consist of two separate silicon devices, the sensor and the read out chip, that are bump-bonded together. They can tolerate radiation up to fluences of $5 \times 10^{1} 5 \mathrm{neq} / \mathrm{cm}^{2}$ and operate at rates up to $300 \mathrm{MHz} / \mathrm{cm}^{2}$ using readout chips created with $130 \mathrm{~nm}$ CMOS technology. For HL-LHC the rate and dose requirements will approximately triple. A readout chip that can handle these requirements would need to use smaller feature sizes (65nm CMOS) and contain some 500 million transistors. The design of such a chip would be a large project that requires international collaboration.

In monolithic active pixel sensors (MAPS) sensor and readout circuitry are implanted in the same Si wafer, providing a single chip solution without bump bonding[14]. They provide the potential for including more functions, e.g., zero suppression, at the pixel level. MAPS have less mass than hybrid pixel detectors, lower capacitance and can be made highly granular and thinned to about $50 \mu \mathrm{m}$ thickness. Finer pixelization will improve spatial resolution and two-track separation. At this thickness the sensors no longer dominate the 
mass in the tracker and cables, support, and cooling services become important. Sensor stitching can further reduce the mass of tracking detectors by routing signals and clocks through metal lines on the chip that are wirebonded between chips. 3D integration would allow combination of sensor and readout chip with different feature sizes/technologies[15].

An alternative approach to reducing the effective thickness are 3D pixel sensors[16]. Planar sensors collect charge in implants on the sensor surface. 3D sensors collect charge in implant columns in the bulk material. The depletion depth is now equal to the distance between implant columns and can be made much smaller than the sensor thickness. With smaller depletion depth these sensors can collect charge faster, have lower leakage current, lower lower depletion voltage, lower power dissipation, and are more radiation tolerant.

A further step are 4D ultra-fast silicon detectors which combine precise spatial resolution with ps time resolution based on silicon thinned to $\approx 5 \mu \mathrm{m}$ to reduce charge collection time[17]. Sensors this thin do not generate enough charge to guarantee near $100 \%$ hit efficiency. Therefore the charge must be increased using charge multiplication in the bulk of the sensor. This can be achieved by tuning the depth doping profile of the bulk material to create a low resistivity region just below the pixel implant. This creates a high field region in which a moderate charge multiplication gain can be achieved. Readout systems to match sensor rate, segmentation, and time measurement capabilities need to be developed. Significant R\&D is still required in the areas of wafer processing options (n-bulk vs p-bulk, planar vs 3D sensors, epitaxial vs float zone) and depth and lateral doping profile.

Diamond sensors also hold promise for the development of radiation hard detectors. Chemical vapor deposition (CVD) diamond has a band gap of $5.5 \mathrm{eV}$ (silicon: $1.1 \mathrm{eV}$ ) and a displacement energy of $42 \mathrm{eV} /$ atom (silicon: $15 \mathrm{eV}$ ). Thus, diamond is expected to be intrinsically more resistant to damage by ionizing radiation and does not require extensive cooling. Another attractive features is that diamond is a low Z material. The flip side is that diamond only generates $60 \%$ as many charge carriers as silicon[18].

Some of the above technologies can be combined to develop new techniques to produce large area, low cost pixelated tracking detectors[19]. These utilize wafer-scale 3D electronics and sensor technologies currently being developed in industry. Readout chips and sensors can be connected at the wafer level using through silicon vias and oxide bonding. Active edge technologies reduce dead areas at the sensor edges, allowing the assembly of large detector modules by tiling the bonded chip-sensor assemblies. This can result in fully active sensor/readout chip tiles which can be assembled into large area arrays with good yield and minimal dead area.

Finally, support, cooling, and power are also in need of R\&D. Next generation trackers will have increased power density, high channel count, high speed data links, and be exposed to high radiation doses. They require efficient cooling and low mass support and services. Current R\&D directions are the development of new materials which are low Z, stiff, thermally conductive, and radiation hard. Possible candidates are carbon foams/fibers and ceramics. The trend is towards multifunction structures that combine mechanical support, cooling and power services in one structure[20]. Radiation tolerant DC/DC converters that can operate in magnetic fields are also a current R\&D direction to reduce power dissipation by bringing in power at higher voltage and reducing it on detector to the required chip supply voltages[21]. 


\subsection{High resolution hadron calorimeters}

In order to separate hadronic $\mathrm{W}$ and $\mathrm{Z}$ boson decays a jet energy resolution of $\sigma \leq 30 \% \sqrt{E}$ is required. The resolution of jet energy measurements is limited by fluctuations in the fractions of the total jet energy carried by electrons and photons, neutral hadrons, and charged hadrons.

One approach to reduce the effect of these fluctuations is to build compensating calorimeters that have the same response for the electromagnetic and hadronic components of the shower. The disadvantage is that the compensation relies on neutrons liberated in the material which is a slow process. Therefore the signal has to be integrated over a rather long time to achieve the compensating effect which is not compatible with the bunch crossing times at current and future energy frontier facilities.

Another approach are dual readout calorimeters[22]. These measure both Cerenkov light and scintillation light. The Cerenkov light originates largely from the electromagnetic component of the shower whereas the scintillation light comes from both the hadronic and electromagnetic components. Measuring both components allows the determination of the electromagnetic to hadronic ratio on a jet-by-jet basis. The jet energy can then be corrected depending on this ratio. The theoretical limit for the resolution that can be achieved in this way is $\sigma \leq 15 \% \sqrt{E}$. This principle can be implemented with sampling calorimeters, e.g. using $\mathrm{Pb} / \mathrm{Cu}+$ scintillating fibers, or with homogeneous crystal calorimeters. The latter require a dense and economical material to make a hadron calorimeter feasible[23, 24].

Light can be piped out of the calorimeter to photosensors using optical fibers. A drawback is that these fibers are often radiation soft. The development of radiation hard fibers is therefore necessary. An example for such $R \& D$ is the development of intrinsically radiation hard claddings with low refractive index from nanoporous alumina[25].

For dual readout efficient UV photodetectors are needed. R\&D is being carried out on Silicon Photomultipliers (SiPM)[26]. These are Geiger-mode avalanche photodiodes. They operate with low power, low voltage, and have low noise. They are compact and have excellent timing resolution. They are insensitive to magnetic fields. As they are made of silicon they are sensitive to radiation and need to be cooled after radiation exposure to keep the leakage current down. GaAs or InGaAs maybe an alternative material. Silicon also has small attenuation length for UV light. Silicon Carbide with a bandgap of $3.2 \mathrm{eV}$ is promising for detecting Cerenkov light.

A different approach is to use a particle flow algorithm to reconstruct individual particles in the shower and apply particle specific corrections. Charged particles can best be measured in the tracker and photons in the electromagnetic calorimeter so that only neutral hadrons have to be measured in the hadron calorimeter. This approach is planned for $\mathrm{e}^{+} \mathrm{e}^{-}$collider detectors. Its efficient implementation requires an imaging calorimeter with high granularity that provides a detailed image of shower[27].

Micro-pattern gas detectors are a possible technology to achieve a sufficiently finely pixelated calorimeter readout[28]. These detectors potentially can provide low cost, large area detectors with high granularity that are fast and radiation hard. Examples are plasma panel sensors (PPS) which resemble plasma-TV display panels, modified to detect gas ionization in the individual cells[29], resistive plate chambers (RPC)[30], flat panel microchannels[31], gas electron multipliers (GEMs), and micromegas. 


\subsection{High-rate muon systems}

Muon detection systems typically form the outermost subdetector layer in high-energy physics experiments, often covering areas of thousands of square meters in large-scale experiments. Consequently, economic construction of such subdetector systems is mandatory. At the same time, muon rates and radiation loads that need to be handled by muon systems in hadron collider experiments are increasing, in particular in the forward direction. For example, muon rates around few to tens of $\mathrm{kHz} / \mathrm{cm}^{2}$ are expected at the HL-LHC. Micro-Pattern Gas Detectors (MPGDs) such as Gas Electron Multipliers (GEMs) and Micromegas have been proven in small-scale tracking applications to handle rates above $\mathrm{MHz} / \mathrm{cm}^{2}$ with spatial resolutions of about 50 microns (for normally incident charged particles) and timing resolutions of a few ns. Recent R\&D efforts by the ATLAS, CMS, and RD51 collaborations have succeeded in scaling up this class of radiation-hard, low-mass detectors to the meter-scale, making MPGDs excellent candidates for robust muon detection at the EF.

Development of cost-effective MPGD mass production techniques and establishment of an industry base with MPGD mass production capabilities within the US are of interest to facilitate the application of MPGDs for large muon detector systems at the EF. Additional research on alternative production techniques such as additive manufacturing (3D printing) for detection elements and possibly entire detectors, as well as the development of advanced anode patterns to reduce the number of required electronics channels have the potential for reducing the cost of MPGD-based detector systems further [33].

\section{Conclusion}

In order to realize our physics goals, we need to invest in technology R\&D. The challenges at energy frontier facilities will be substantial. Detectors at all facilities will require increased spatial pixelization and time resolution. They will have to deal with increasing data volumes. There are many ideas for instrumentation that can address these challenges.

We need a stable mechanism that provides funding to develop them into viable technologies. An investment into technology R\&D will carry many dividends in the future. Detector technologies that enable the construction of large-scale detectors at affordable cost will increase the depth and breadth of particle physics research that can be carried out within a certain budget. Support for technology R\&D will enable us to explore particle physics more effectively but it will also increase the benefits of the field of particle physics to society, in the form of technology spinoffs and the injection of highly trained, technically skilled individuals into the work force.

\section{References}

[1] European Strategy for Particle Physics Preparatory Group: Physics Briefing Book, CERN-ESG-005.

[2] M. Stanitzki, "Instrumentation challenges for $\mathrm{e}^{+} \mathrm{e}^{-}$colliders", presented at Snowmass meeting, Minneapolis, Aug 1, 2013. 
[3] L. Linssen for the CLIC detector and physics study: "Replies to questions from Instrumentation Frontier to Energy Frontier".

[4] Anna Mazzacane, "Detector challenges for $\mu^{+} \mu^{-}$, presented at Snowmass meeting, Minneapolis, Aug 1, 2013.

[5] V. Di Benedetto, C. Gatto, R. Lipton, A. Mazzacane, N.V. Mokhov, A. Para, S.I.Striganov, N.K. Terentiev, H. Wenzel: "Tracker and Vertex Detector for a Muon Collider", white paper submitted to the instrumentation frontier working group.

[6] "Emerging Optical Link Technologies for HEP", white paper submitted to the instrumentation frontier working group.

[7] "High speed, massively parallel, ATCA based Data Acquisition Systems using modular components", white paper submitted to the instrumentation frontier working group.

[8] W. Smith: "Triggers for hadron colliders at the energy frontier", white paper submitted to the instrumentation frontier working group.

[9] G. Brooijmans, H. Evans, A. Seiden: "Atlas Upgrade Instrumentation", white paper submitted to the instrumentation frontier working group.

[10] E. Grunendahl, M. Johnson, R. Lipton, T. Liu, A. Ryd, L. Spiegel: "Level 1 Track Triggers at HL-LHC", white paper submitted to the instrumentation frontier working group.

[11] G. De Geronimo, D. Christian, C. Bebek, M. Garcia-Sciveres, H. Von der Lippe, G. Haller, A.A. Grillo, M. Newcomer, "Application Specific Integrated Circuits (ASICs) for HEP applications", white paper submitted to the instrumentation frontier working group.

[12] E. Oberla, K. Nishimura: "Future Developments in Gigasample-per-second Waveform Sampling Application Specific Integrated Circuits", white paper submitted to the instrumentation frontier working group.

[13] G. Bolla, M. Garcia-Sciveres: "Hybrid Pixel Detector Challenges for next Generation Frontier Experiments at the LHC", white paper submitted to the instrumentation frontier working group.

[14] M. Battaglia: "Monolithic Pixel Sensors", white paper submitted to the instrumentation frontier working group.

[15] R. Lipton, C. Kenney, S. Parker, L. Spiegel, J. Thom: "Combination of Active Edge and 3D Electronics Technologies", white paper submitted to the instrumentation frontier working group.

[16] C. Da Via, C. Kenney: "3D Architecture Pixel Sensors", white paper submitted to the instrumentation frontier working group. 
[17] H. Sadrozinski: "Ultra-fast Silicon Detectors (4D-UFSD)", white paper submitted to the instrumentation frontier working group.

[18] H. Kagan, W. Trischuk: "Diamond Sensors", white paper submitted to the instrumentation frontier working group.

[19] R. Lipton, G. Deptuch, U. Heintz, M. Johnson, C. Kenney, M. Narian, S. Parker, A. Shenai, L. Spiegel, J. Thom, and Z. Ye, "3D Technologies for Large Area Trackers", white paper submitted to the instrumentation frontier working group.

[20] W. Cooper, C. Haber, D. Lynn: "Low Mass Support and cooling", white paper submitted to the instrumentation frontier working group.

[21] S. Dhawan, R. Sumner, R. Khanna: "Powering Future Particle Physics Detectors", white paper submitted to the instrumentation frontier working group.

[22] RD52 Collaboration: "Hadronic dual-readout calorimetry for high energy colliders", white paper submitted to the instrumentation frontier working group.

[23] ???, "Development of Cost-effective Crystals For Homogenous Hadron Calorimetry", white paper submitted to the instrumentation frontier working group.

[24] Ren-Yuan Zhu, "The Next Generation of Crystal Detectors", white paper submitted to the instrumentation frontier working group.

[25] C. Sanzeni, G. Jennings, D.R. Winn, "Ultra-Low Index and Rad-Hard Total Internal Reflection Claddings for High Numerical Aperture Scintillating, Waveshifting, Cerenkov, or Clear Optical Fibers, Capillaries, and Plates", white paper submitted to the instrumentation frontier working group.

[26] R. Rusack: "Solid State Photodetectors", white paper submitted to the instrumentation frontier working group.

[27] ???, "Imaging Calorimetry", white paper submitted to the instrumentation frontier working group.

[28] J. Yu, M. Hohlmann, V. Polychronakos, and A. White: "Micro-Pattern Gas Detectors for Calorimetry", white paper submitted to the instrumentation frontier working group.

[29] R. Ball, J. R. Beene, Y. Benhammou, E. H. Bentefour, J. W. Chapman, E. Etzion, C. Ferretti, P. S. Friedman, D. S. Levin, M. Ben Moshe, Y. Silver, R. L. Varner, C. Weaverdyck, and B. Zhou: "Plasma Panel Detectors for Ionizing Particles", white paper submitted to the instrumentation frontier working group.

[30] B. Bilki, K. Francis, J. Repond, L. Xia: "Development of Resistive Plate Chambers", white paper submitted to the instrumentation frontier working group.

[31] A. Ronzhin, H. Frisch: "Use of Flat Panel Microchannel Plates in Sampling Calorimeters with Timing", white paper submitted to the instrumentation frontier working group. 
[32] A.Albayrak-Yetkin, B.Bilki, J.Corso, P.Debbins, G.Jennings, V.Khristenko, A.Mestvirisvilli, Y.Onel, I.Schmidt, C.Sanzeni, D.Southwick, D.R.Winn, T.Yetkin, "Secondary Emission Calorimetry: Fast and Radiation-Hard", white paper submitted to the instrumentation frontier working group.

[33] M. Hohlmann, V. Polychronakos, A. White, J. Yu, "Micro-Pattern Gas Detectors for Charged-Particle Tracking and Muon Detection", SNOW13-00025, arXiv:1306.1924 [physics.ins-det]. 


\section{Title: Particle Flow Calorimetry for CMS}

Names: Burak Bilki (ANL and Iowa), Marcello Mannelli (CERN), Yasar Onel (Iowa), José Repond (ANL), Roger Rusack (Minnesota), Lei Xia (ANL)

\section{Time Frame: Long}

Physics Justification: At the High-Luminosity LHC (HL-LHC) identification and measurement of jets in the forward direction will be required to measure the VBF production of the Higgs. This is a crucial measurement for the HL-LHC, as the cross-section for this process is controlled by the Higgs mechanism, and can therefore be used as a confirmation of the mechanism itself and, through deviations to the Standard Model, as a sensitive area to look for manifestations of new physical processes. Another process that will be accessible only at the HL-LHC, due to its low cross section is the Higgs triple coupling. For this the widest possible acceptance is essential.

CMS (the Compact Muon Solenoid) is engaged in the design of a new calorimeter system for the forward direction and one of the concepts under investigation is imaging or particle flow calorimetry. In the studies that have been done for the ILC/CLIC detectors the application of Particle Flow Algorithms (PFAs) has been shown to improve significantly the jet energy resolution. A simpler form of particle flow is presently used as a standard tool for measuring jets in all CMS physics analyses. However, in the design the CMS detector was not optimized for this application. For HL-LHC operation upgrades to the CMS detector are being designed that are optimized for the application of PFAs.

PFAs use a synergy of information from precise tracking upstream and a highly segmented, in both the longitudinal and lateral directions, calorimeter downstream. This approach requires advanced technologies for both the active media and the electronic readout of the calorimeter.

Outstanding Challenges: The forward calorimetry in CMS faces the following major challenges, among others:

1. The Radiation conditions: The active media and its embedded readout electronics will need to withstand the high radiation levels of the forward direction, while preserving their performance. Integrated neutron fluences approaching $10^{16}$ neutrons $/ \mathrm{cm}^{2}$ can be expected in the hottest areas of the detector.

2. Timing: The bunch-crossing rate at the HL-LHC will be $40 \mathrm{MHz}$ and there will be on average 140 events per bunch crossing. This leads to the requirement that the signal should be contained, as much as possible, within the $25 \mathrm{~ns}$ interval. This has consequences for the active media, the electronic readout, and the passive absorber material. A fine time resolution might be required in order to handle the high event pile-up conditions.

3. Pile-up: The number of $\sim 140$ overlapping events every bunch crossing will inject on the order of 1000 low energy particles into the calorimeter per unit of eta-phi space.

4. Other requirements: The detectors should be compact, low cost, and mechanically and electronically compatible with the existing detector.

Imaging Electromagnetic Calorimeters: There has been significant R\&D for high segmentation electromagnetic calorimeters with Silicon or scintillator strips with SiPM readout as active medium. Within the CALICE collaboration, both options were successfully tested with 
small-scale prototypes. However, additional work is needed to address the challenges mentioned above. Other options being evaluated include Crystal calorimetry (challenges being aging and signal pile-up), Shashlik type calorimeters, GEMs, and Secondary Emission Calorimetry (SEC), which is currently being validated in test beams.

Imaging Hadron Calorimeters: Existing imaging hadron calorimetry falls into two categories: i) Scintillator pads with SiPM readout and ii) gaseous detectors, such as Resistive Plate Chambers (RPCs), Micromegas, and Gas Electron Multipliers (GEMs). The scintillator option utilizes an analog readout and the gaseous detectors opt for single bit (=digital) or two-bit (=semi-digital) readout. All concepts have been tested and validated in test beams. Significant $\mathrm{R} \& \mathrm{D}$ work is being pursued by the RPC-based digital and semi-digital calorimetry towards an increased rate capability to comply with the LHC conditions.

\section{Key Motivations for this Detector R\&D:}

$\checkmark$ Development of imaging calorimetry for hadron colliders.

$\checkmark$ Significant improvement in the quality of CMS data with the complete utilization of PFAs in the forward region.

$\checkmark$ Advances in understanding of calorimetry.

$\checkmark$ Application and testing of novel technologies, such as SiPM, large area Micropattern detectors and more. 
Title: Noble Liquid Calorimeters

Time Frame: Present - Intermediate

Physics Justification: Noble liquid calorimeters, such as the ATLAS Liquid Argon (LAr) calorimeter, are advantageous because of the intrinsic radiation hardness of noble liquids and the fact that they provide a uniform response. The design of the ATLAS LAr calorimeter as a sampling calorimeter with an accordion geometry provides high readout speed and minimal dead space. The excellent performance of the LAr calorimeter was an essential component for the discovery of the Higgs-like boson in ATLAS.

Technical Capabilities: Of the ATLAS LAr calorimeter's $\sim 180,000$ channels, $>99.9 \%$ are currently operational and it has excellent timing resolution ( $0.14 \mathrm{~ns}$ measured in the electromagnetic barrel calorimeter in $\sqrt{\mathrm{s}}_{\mathrm{s}}=8 \mathrm{TeV}$ proton-proton collisions). The electromagnetic scale at the $\mathrm{Z}$ boson mass is known to $\sim 0.3 \%$, the linearity is known better than $1 \%$, and the constant term of the resolution is $\sim 1 \%(2.5 \%)$ for $|\eta|<1.37(1.37<|\eta|<1.8)$. The mass resolution and stability of the calorimeter response has been shown to be insensitive to the number of interactions per bunch crossing throughout data-taking in 2012.

Technical Requirements: For the High-Luminosity LHC, the Forward Calorimeter (FCal) in ATLAS is of particular concern. At the HL-LHC, we expect space charge effects to degrade the signal, the potential across the gaps to sag due to excessive current drawn from the high voltage supply, and heating on the verge of boiling the liquid argon. Three potential options are being considered: (0) do nothing, (1) replace the present FCal with narrower LAr gaps, or (2) insert a new, small module in front of the present FCal. Option (1) will only be considered if the cryostat will be opened to replace the hadronic endcap electronics. A potential design for option (2) is a 'miniFCal' with 9 rings of $1-\mathrm{cm}$ square diamond wafers interspersed with copper absorbers.

Industrial Involvement: N/A

Key Motivations for this Detector R\&D:

$\checkmark$ Maintains the excellent LAr calorimeter performance at the HL-LHC 


\section{Topic: Hadronic dual-readout calorimetry for high energy colliders}

\section{Group: RD52 at CERN}

Justification: The capability of a hadronic calorimeter to measure the invariant mass of two hadronic jets to a precision of $2-3 \mathrm{GeV}$, and thereby explicitly separate $W \rightarrow q_{i} \overline{q_{j}}$ from $Z \rightarrow q \bar{q}$ in collider experiments, requires that individual jets, and the spray of hadrons of which they are composed, be directly measured to a stochastic precision of

$$
\frac{\sigma}{E} \approx \frac{30 \%}{\sqrt{E}} \oplus[\text { small constant term }] \quad \text { (Hadronic energy). }
$$

This raw hadronic energy precision was achieved in the SPACAL module tested at CERN, which consisted of scintillating fibers embedded in a $\mathrm{Pb}$ matrix at the compensating ratio of scintillator-to- $\mathrm{Pb}$ of $2.4 \%$. SPACAL has been emulated in several experiments and is still a favored calorimeter technology in many applications.

Current R\&D: However, the Pb-fiber compensating ratio resulted in less-than-ideal electromagnetic energy resolution to accompany the excellent hadronic energy resolution and, in addition, the calorimeter mass required 20 tonnes of $\mathrm{Pb}$ and 50-100 ns of signal integration time to fully measure the neutrons released from the $\mathrm{Pb}$ nuclei that are necessary for compensation. The invention of dual-readout calorimeters solves these problems at once: a wide range of absorber-to-sensor ratios are usable, the electromagnetic energy resolution can easily be near

$$
\frac{\sigma}{E} \approx \frac{10 \%}{\sqrt{E}} \quad \text { (Electromagnetic energy) }
$$

the response is Gaussian, and the mean response is linear in hadronic energy after a simple calibration with electrons at just one energy. The fundamental optimum hadronic energy resolution is somewhere near

$$
\frac{\sigma}{E} \approx \frac{15 \%}{\sqrt{E}} \quad \text { (Theoretical limit for hadronic energy) }
$$

limited by unmeasurable fluctuations. The scientific goal of the RD52 group is to measure the fundamental limitations to energy resolution of hadronic calorimeters, and thus open the door to an optimum calorimeter for future experiments.

Near-term R\&D: The DREAM collaboration (CERN project RD52) is building and testing larger hadronic calorimeter modules of both $\mathrm{Pb}$ and $\mathrm{Cu}$ absorbers with the goal of designing and testing multi-ton hadronic calorimeters from which the mean leakage fluctuations are less that $1 \%$. Along with these modules (both $\mathrm{Pb}$-fiber and $\mathrm{Cu}$-fiber), we will increase the Cerenkov photo-electron yield to about $100 \mathrm{Cpe} / \mathrm{GeV}$, make projective modules for a $4 \pi$ calorimeter; test SiPMs as photo converters on some modules; use $5 \mathrm{GHz}$ digitization readout on all channels, both scintillation and Cerenkov; and built a module with a high fraction of tungsten, $\mathrm{W}$, to achieve a module with an absorber density near $15-16 \mathrm{~g} / \mathrm{cm}^{3}$. All of the above implementations will improve the excellent dual-readout characteristics of the present tested modules. RD52 is a generic instrumentation project currently not connected to any experiment. 


\title{
Another Detector for the International Linear Collider
}

\author{
Hanna Arnold ${ }^{1}$, Aaron Bazal ${ }^{1}$, Franco Bedeschi $^{2}$, Robert Basili $^{1}$, \\ Franco Grancagnolo ${ }^{3}$, John Hauptman ${ }^{1}$, Fedor Ignatov ${ }^{6}$, Sehwook Lee ${ }^{7}$, \\ Alexander Mikhailichenko ${ }^{4}$, Tim Overton ${ }^{1}$, Sung Keun Park ${ }^{5}$, Andrew Priest ${ }^{1}$, \\ Fabrizio Scuri ${ }^{2}$, Giovanni Tassielli ${ }^{3}$, Richard Wigmans ${ }^{7}$, Bingzhe Zhao ${ }^{1}$, \\ Gabriella Gaudio (Pavia), Nural Akchurin ${ }^{5}$, \\ Marcel Demarteau (ANL), Caroline Milstene, Ryuji Yamada (Fermilab) * \\ 1 - Iowa State University, Department of Physics and Astronomy, Ames, IA 50011 USA \\ 2 - INFN, Sezione di Pisa, via Livornese 582/a, I-56010 S. Piero a Grado, Pisa, Italy \\ 3 - INFN and Dipartimento di Fisica, via Lecce-Arnesano, 73100, Lecce, Italy \\ 4- Laboratory for Elementary Particle Physics, Cornell University, Ithaca, NY 14853-5001 USA \\ 5 - Korea University, Department of Physics, Anam-dong, Seoul 136-701, Korea \\ 6 - Budker Institute of Nuclear Physics, Lavrentyeva-11, RU-630 090 Novosibirsk, Russia \\ 7 - Texas Tech University, Department of Physics, Lubbock, TX 79409-1051 USA
}

\begin{abstract}
We describe another detector ${ }^{\mathrm{a}}$ designed for the International Linear Collider based on several tested instrumentation innovations in order to achieve the necessary experimental goal of a detecter that is 2-to-10 times better than the already excellent SLC and LEP detectors, in particular, (1) dual-readout calorimeter system based on the RD52/DREAM measurements at CERN, (2) a cluster-counting drift chamber based on the successful KLOE chamber at Frascati, and (3) a second solenoid to return the magnetic flux without iron. A high-performance pixel vertex chamber is presently undefined. We discuss particle identification, momentum and energy resolutions, and the machine-detector interface that together offer the possibility of a very high-performance detector for $e^{+} e^{-}$physics up to $\sqrt{s}=1 \mathrm{TeV}$.
\end{abstract}

\footnotetext{
${ }^{*}$ This work has been supported by the US Department of Energy and by INFN, Italy.

${ }^{a}$ We can generate names and acronyms, too: AUDAX, audacious, bold (Latin); CAD, Compact Advanced Detector; or, CLD, Compact Lightweight Detector.
}

Community Summer Study, Snowmass on d' Miss'sip, Minneapolis, MN, USA, 29Jul-9Aug 2013 


\section{Introduction}

The physics reach of a new high energy $e^{+} e^{-}$linear collider requires [1] the measurement and identification of all known partons of the standard model $(e, \mu, \tau, u d s, c, b, W, Z, \gamma, \nu)$ including the hadronic decays of the gauge bosons, $W \rightarrow q \bar{q}$ and $Z \rightarrow q \bar{q}$ and, by subtraction, the missing neutrinos in $W \rightarrow e \nu, W \rightarrow \mu \nu, \tau \rightarrow \ell \nu_{\ell} \nu_{\tau}$ and $\tau \rightarrow h \nu_{\tau}$ decays so that kinematically over-constrained final states can be achieved. A main benchmark process is

$$
e^{+} e^{-} \rightarrow H^{0} Z^{0} \rightarrow(\text { anything })+\mu^{+} \mu^{-}
$$

in which the two $\mu$ s are measured in the tracking system and the Higgs is seen in the missing mass distribution against the $\mu^{+} \mu^{-}$system. This decay mode stresses the tracking system. A momentum resolution of $\sigma_{p} / p^{2} \approx 4 \times 10^{-5}(\mathrm{GeV} / \mathrm{c})^{-1}$ is required for a desired Higgs mass resolution of $150 \mathrm{MeV} / \mathrm{c}^{2}$ in a $500 \mathrm{fb}^{-1}$ data sample. There are three main technologies under study to achieve this performance: a 5-layer silicon strip tracker (SID), a TPC with sophisticated high-precision end planes (ILD), and a cluster-counting drift chamber (this detector) [2].

This same final state can be studied for $Z \rightarrow q \bar{q}$ decays which are 20 times more

$$
e^{+} e^{-} \rightarrow H^{0} Z^{0} \rightarrow(\text { anything })+q+\bar{q} \rightarrow(\text { anything })+\text { jet }+ \text { jet }
$$

plentiful than $Z \rightarrow \mu^{+} \mu^{-}$decays but less distinct experimentally. This mode stresses the hadronic calorimeters. In addition, those processes that produce $W$ and $Z$ bosons either by production $\left(e^{+} e^{-} \rightarrow W^{+} W^{-}, Z^{0} Z^{0}, H H Z\right)$ or by decay $\left(H \rightarrow W^{+} W^{-}\right)$, will rely critically on the direct mass resolution on $Z \rightarrow q \bar{q}$ and $W \rightarrow q \bar{q}$ decays, and these processes demand that the calorimeter energy resolution be $\sigma_{E} / E \approx 30 \% / \sqrt{E}$ with a constant term less than $1 \%$. There are two main technologies under study: a highly segmented calorimeter volume with approximately $(1 \mathrm{~cm})^{3}$ channels for the implementation of Particle Flow Analysis (PFA) algorithms (SID and ILD), and dual-readout optical calorimeters that measure both scintillation and Cerenkov light (this detector) [3].

The identification of $b$ and $c$ quark and $\tau$ lepton decays is critical to good physics since these massive particles are a gateway to the decays of more massive, and possibly new, particles [4]. The measurement of their respective decay lengths places stringent conditions on the spatial precision of a vertex chamber and how close it can be to the beam interaction spot. Typically, a spatial resolution

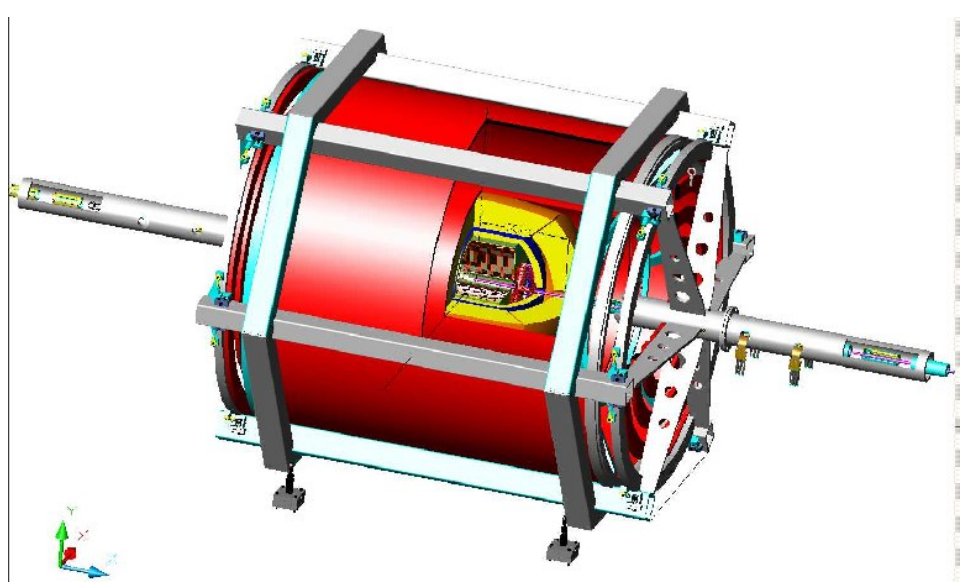

Figure 1: This alternative detector. of $\sigma \approx 5 \mu \mathrm{m}$ is desired, 
with large solid angular coverage. The inner radius is limited by the debris from beamstrahlung that is only suppressed by the axial tracking magnetic field; the uncharged debris cannot be suppressed, but becomes nearly invisible in our He-based tracking system which presents a negligibly thin absorber for the $\gamma$ 's from the violent beam crossing.

The region beyond the calorimeter is reserved for, typically, a superconducting solenoid to establish the tracking field and a hadron absorber or muon filter to assist muon tagging. There are two main types of muon systems under study: an iron absorber interspersed with tracking chambers to measure the trajectories of penetrating tracks (SID and ILD), and an iron-free design in which the magnetic flux is returned by a second outer solenoid (this detector).

We are skeptical of several aspects of the SID and ILD detectors, viz., power pulsing of a complex silicon tracking system, the analysis complexity of a particle flow calorimeter, positive ion loading in a TPC, and a general lack of particle identification measurements.

\section{An Alternative Detector}

We have introduced new ideas and instruments in order to achieve the resolution requirements needed for ILC physics studies [5,6]. With the exception of the vertex chamber, we designed a powerful detector that dramatically differs from the detectors at SLC and LEP and from the two ILC concept detectors, ILD and SID, in all three major detector systems: the tracking, the calorimeter and the muon system. This detector is displayed in Figure 1, showing the beam transport through the final focus, the dual solenoids (red), fiber dualreadout calorimeters (yellow), the tracking chamber, and the vertex chamber. The muon tracking is in the annulus between the solenoids. A forward toroid for high precision forward tracking in under current study in this figure. This detector is about 1/10 the mass of a conventional detector with an iron yoke flux return.

\subsection{Tracking by cluster counting in a low-mass He-based drift chamber}

The gaseous central tracker is a cluster-counting drift chamber modeled on the successful KLOE main tracking chamber $[2,6,7]$. This drift chamber $(\mathrm{CluCou})$ maintains very low multiple scattering due to a He-based gas and aluminum wires in the tracking volume and utilizes carbon fiber end plates. Forward tracks (beyond $\cos \theta \approx 0.7$ ) which penetrate the wire support frame and electronics pass through only about $15-20 \% X_{0}$ of material. The ultra-low mass of the tracker in the central region directly improves momentum resolution in the multiple scattering dominated

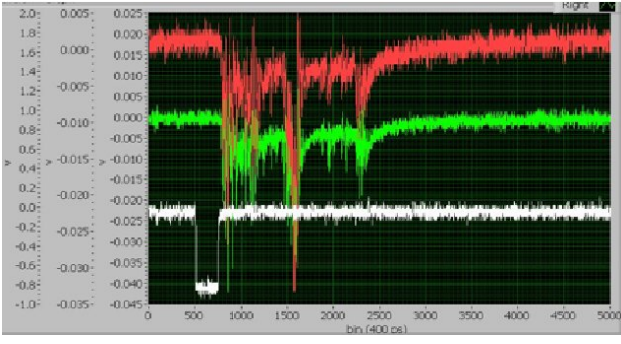

Figure 2: Ionization cluster data. region below $10 \mathrm{GeV} / \mathrm{c}$. The $\mathrm{He}$ gas has a low drift velocity which allows a new cluster counting technique that clocks in individual ionization clusters on every wire, providing an estimated 40 micron spatial resolution per point, a $d E / d x$ resolution near $3 \%, z$-coordinate information on each track segment through an effective dip angle measurement, and a layout made exclusively of super-layers with alternated opposite sign stereo angles. The maximum drift time in each cell is less than the 
300ns beam crossing interval, so this chamber sees only one crossing per readout. Data from a test of cluster counting are shown in Figure 2.

The critical issues of occupancy and two-track resolution have been simulated for ILC events and expected machine and event backgrounds, and direct $\mathrm{GHz}$ cluster counting experiments are being performed. This chamber has timing and pattern recognition capabilities midway between the faster, higher precision silicon tracker of SID and the slower, full 3dimensional imaging TPC of ILD, and is superior to both with respect to its low multiple scattering.

The low-mass of the tracking medium, the multiplicity of point measurements (about 200 ), and the point spatial precision allow this chamber to reach $\sigma_{p} / p^{2} \approx 5 \times 10^{-5}(\mathrm{GeV} / \mathrm{c})^{-1}$ at high momenta, and to maintain good momentum resolution down to low momenta.

\subsection{Calorimetry by dual-readout of scintillation and Ĉerenkov light}

The calorimeter is a spatially finegrained dual-readout fiber sampling calorimeter augmented with the ability to measure the neutron content of a shower[6]. The dual fibers are sensitive to scintillation and Cerenkov radiation, respectively, for separation of the hadronic and electromagnetic components of hadronic showers [8], and since fluctuations in the EM fraction, i.e., fluctuations in $\pi^{0}$ production relative to $\pi^{ \pm}$, are largely responsible for poor hadronic energy resolution, the DREAM module achieved a substantial improvement in hadronic calorimetry. The energy resolution of the tested DREAM

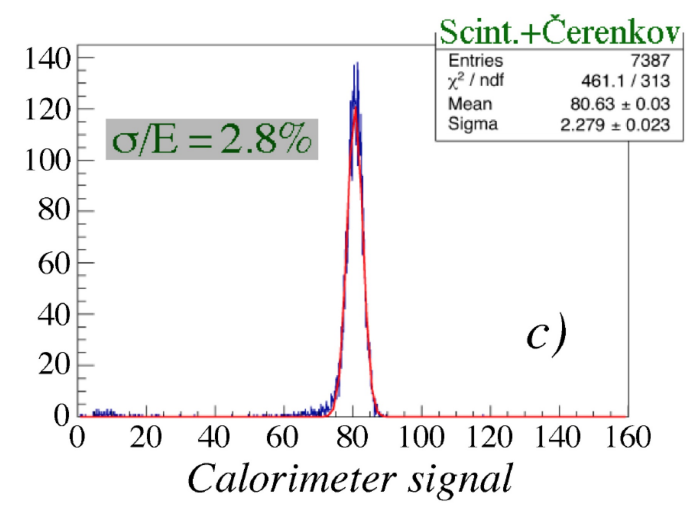

Figure 3: DREAM electromagnetic energy resolution.[10]
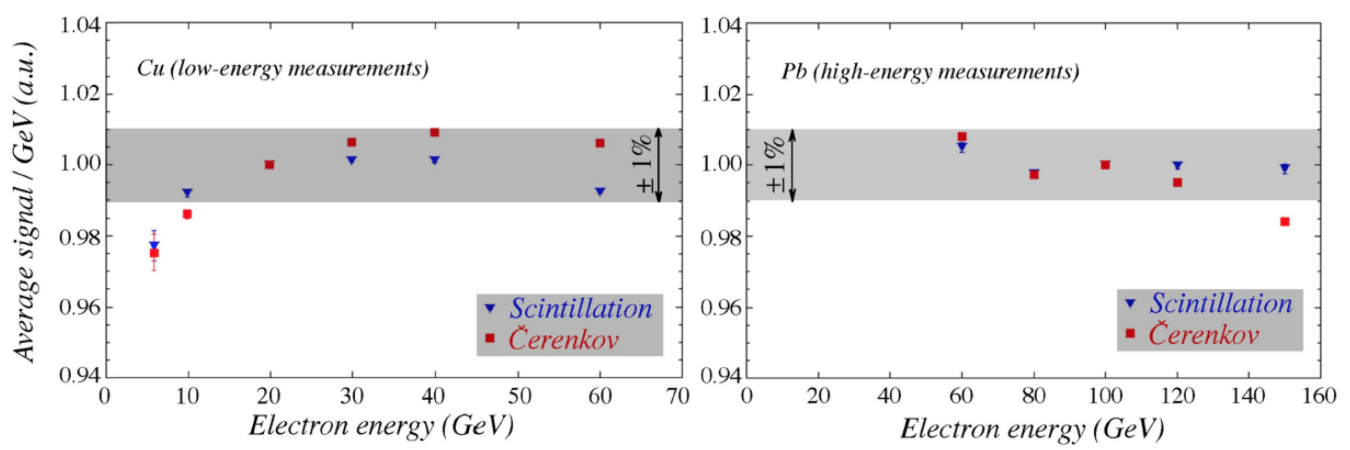

Figure 4: Measured response of the dual readout calorimeters of RD52 for hadrons from 5 to $150 \mathrm{GeV}$. Each module was calibrated only with $60 \mathrm{GeV}$ electrons. The $2 \%$ drop at low beam energy is due to material in the beam chambers upstream.

Community Summer Study, Snowmass on d' Miss'sip, Minneapolis, MN, USA, 29Jul-9Aug 2013 
calorimeter has been surpassed in the new RD52 modules with finer spatial sampling, neutron detection for the measurement of fluctuations in binding energy losses[?], and a larger volume test module to reduce leakage fluctuations. The calorimeter modules will have fibers up to their edges, and will be constructed for sub-millimeter close packing, with signal extraction at the outer radius so that the calorimeter system will approach full coverage without cracks. The electromagnetic energy resolution, spatial resolution, and particle identification are so good in the fiber system than a separate crystal dual-readout in not desired.

The fiber calorimeter shows promise of excellent energy resolution on hadrons and jets, as detailed in recent progress reports. We expect to achieve a hadronic energy resolution near $1-2 \%$ on a full-sized calorimeter,

$$
\frac{\sigma}{E} \approx 1-2 \% \quad \text { (hadronic energy resolution on jets) }
$$

at high energies, including a small constant term (which has many sources).

Most importantly, the hadronic response of this dual-readout calorimeter is demonstrated to be linear in hadronic energy from 20 to $300 \mathrm{GeV}$ having been calibrated with electrons at only one energy Fig. 4. This is a critical advantage at the ILC where calibration with $45 \mathrm{GeV}$ electrons from $Z$ decay will suffice to maintain the energy scale up to 10 times this energy for physics.

Finally, we expect to construct a small tungsten dual-readout module identical in geometry to our copper and lead modules. Such a development would retain the good features of dual-readout but reduce the calorimeter depth to 1.5 meters, resulting in enormous economies in a large $4 \pi$ collider detector.

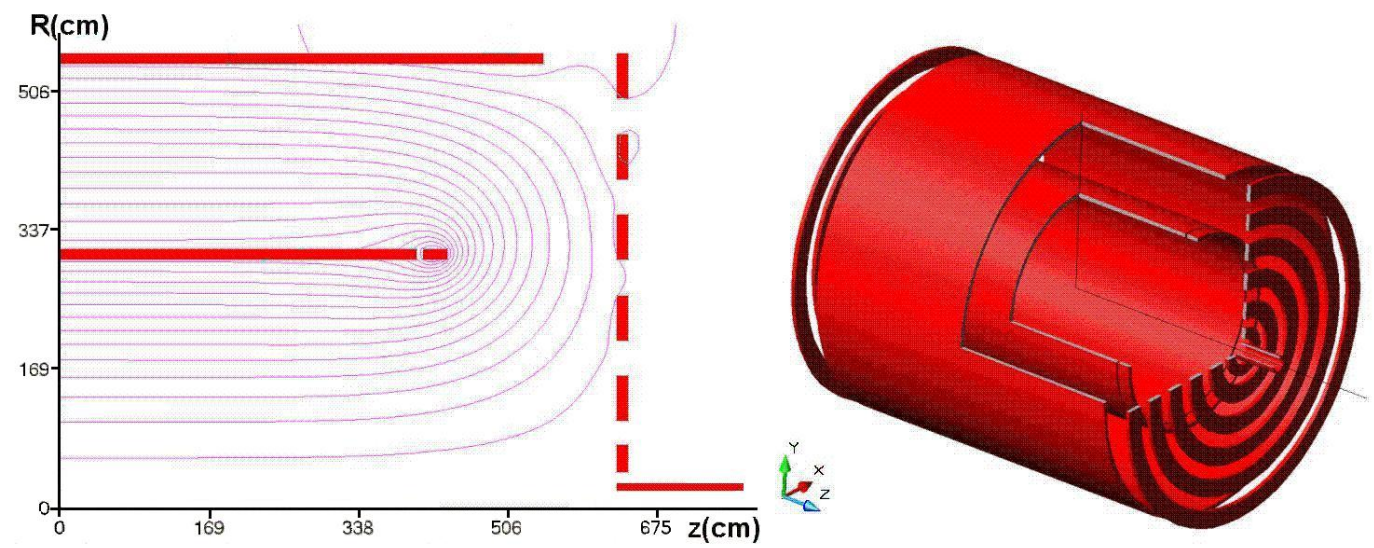

Figure 5: Drawings showing the two solenoids and the "wall of coils" that redirects the field out radially, and the resulting field lines in an $r-z$ view. This field is uniform to $1 \%$ at $3.5 \mathrm{~T}$ in the tracking region, and also uniform and smooth at $-1.5 \mathrm{~T}$ in the muon tracking annulus between the solenoids.

Community Summer Study, Snowmass on d' Miss'sip, Minneapolis, MN, USA, 29Jul-9Aug 2013 


\subsection{The dual solenoid magnetic field configuration}

The muon system utilizes a dual-solenoid magnetic field configuration in which the flux from the inner solenoid is returned through the annulus between this inner solenoid and an outer solenoid oppositely driven with a smaller turn density [12]. The magnetic field in the volume between the two solenoids will back-bend muons (and punch-through pions) which have penetrated the calorimeter and allow, with the addition of tracking chambers, a second momentum measurement. This will achieve high precision without the limitation of multiple scattering in $\mathrm{Fe}$ that fundamentally limits momentum resolution in conventional muon systems to $10 \%$. High spatial precision drift tubes with cluster counting electronics are used to measure tracks in this volume [13]. The dual-solenoid field is terminated by a novel "wall of coils" that provides muon bending down to small angles $(\cos \theta \approx 0.975)$ and also allows good control of the magnetic environment on and near the beam line. The design is illustrated in Fig 5 .

The path integral of the field in the annulus for a muon from the origin is about $3 \mathrm{~T} \cdot \mathrm{m}$ over $0<\cos \theta<0.85$ and remains larger than $0.5 \mathrm{~T} \cdot \mathrm{m}$ out to $\cos \theta=0.975$, allowing both good momentum resolution and low-momentum acceptance over almost all of $4 \pi$ [13].

For isolated tracks, the dual readout calorimeter independently provides a unique identification of muons relative to pions with a background track rejection of $10^{4}$, or better, for high energies through its separate measurements of ionization and radiative energy losses, Fig. ??. This will be important for the $\tau^{ \pm}$decays from $H^{0}$ and $W, Z$ leptonic decays.

\section{Particle Identification}

The capability to identify standard model partons $(e, \mu, \tau, u / d / s$, $c, b, t, W, Z, \gamma)$ is equivalent to increased luminosity with larger, and less ambiguous, data ensembles available for physics analysis.

$\pi^{0} \rightarrow \gamma \gamma$ separation and reconstruction The dual-readout crystals can be made small, about $1 \mathrm{~cm} \times 1 \mathrm{~cm}$ or $2 \mathrm{~cm} \times 2 \mathrm{~cm}$, and with reconstruction using shower shapes in the crystals we estimate that $\pi^{0} \rightarrow \gamma \gamma$ can be reconstructed up to about $E_{\pi^{0}} \sim 20 \mathrm{GeV}$, which is high enough to reconstruct the important decay $\tau \rightarrow$ $\rho \nu \rightarrow \pi^{ \pm} \pi^{0} \nu \rightarrow \pi^{ \pm} \gamma \gamma \nu$ to be used as a spin analyzer in the decays of the $125 \mathrm{GeV} / \mathrm{c}^{2} H^{0}$, etc.

$e, \pi, K, p$ separation by $d E / d x$ at lower momenta The cluster

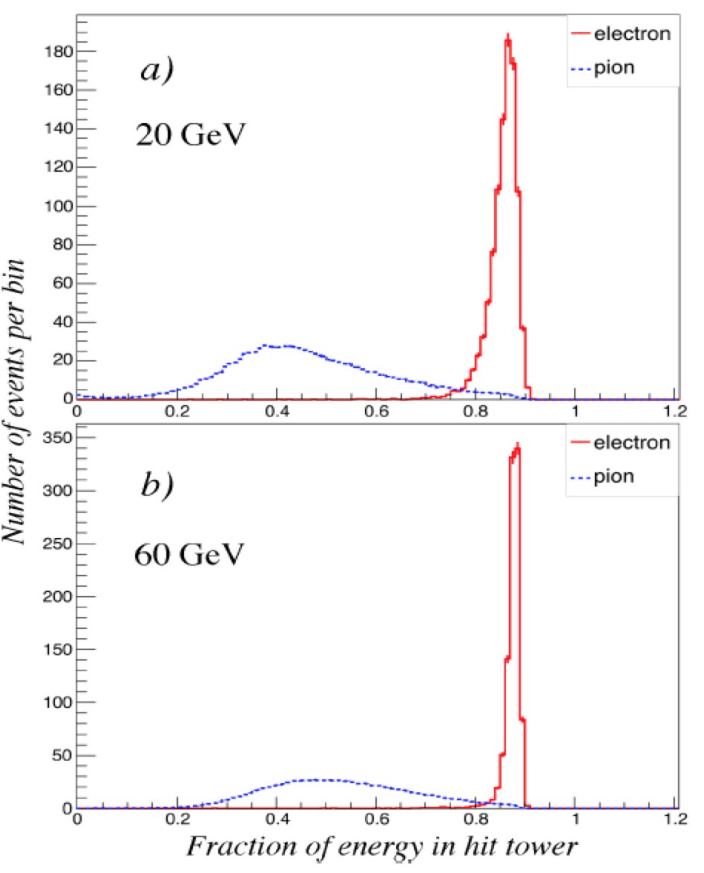

Figure 6: (a) The fraction of energy deposited in the

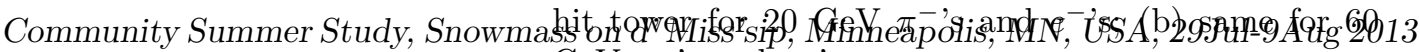
$\mathrm{GeV} \pi^{-}$'s and $e^{- \text {'s. }}$. 
counting central tracking chamber has the added benefit of an excellent energy loss measurement without a Landau tail since clusters are counted as Poisson. We anticipate $3 \%$ or better resolution in $d E / d x$ as an analysis tool in, for example, $b$ physics where a large fraction of charged tracks are below a few $\mathrm{GeV} / \mathrm{c}$.

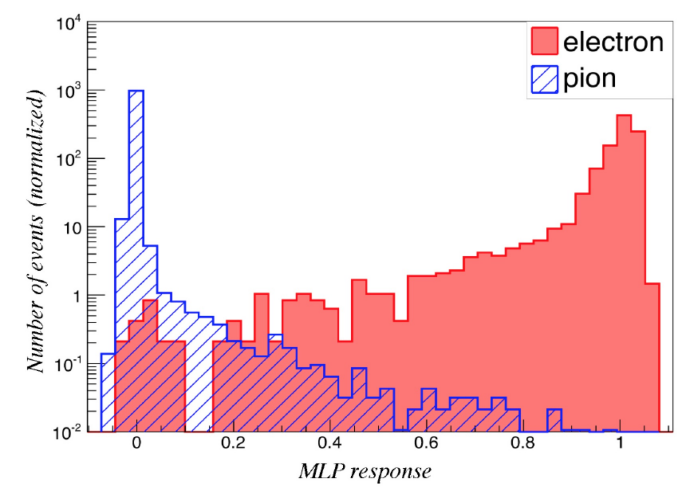

Figure 7: A multi-variate discriminant, MLP, of $e^{ \pm}$ from $\pi^{ \pm}$using the fraction of Fig. 6, an S/C measurement, and a time-depth measurement. $\pi^{ \pm}$rejections of $99.9 \%$ for $e^{ \pm}$efficiencies of $99.9 \%$ are achievable. e separation from $\pi^{ \pm}$and jets $(j)$ Dual-readout allows many measurement to discriminate $e^{ \pm}$from $\pi^{ \pm}$. A lateral shape discriminant [11] in Fig. 6 is very effective for narrow fiber channels; when combined with depth-time information and a scintillation-toĈerenkov measurement, discrimination reaches levels of 1000-to-1, Fig. 7.

Direct use of the scintillation vs. the Cerenkov responses, both for the overall shower and in individual channels, provides further discrimination: just as the overall shower $C$ vs. $S$ response fluctuates in hadronic showers, so do the individual channels of $\pi^{ \pm}$showers.

The statistic

$$
\sigma_{Q-S}=\frac{1}{N} \sum_{i=1}^{N}\left(Q_{i}-S_{i}\right)^{2}
$$

is a measure of these channel-to-channel fluctuations. For $100 \mathrm{GeV} e$ this $\sigma^{e} \approx 0.2 \mathrm{GeV}^{2}$, and for $\pi^{ \pm} \sigma^{\pi} \approx 10 \mathrm{GeV}^{2}$, yielding a rejection of $\pi^{ \pm}$against $e$ of about 50 .

The time history of the scintillating signal contains independent information, in particular, the neutrons generated in the $\pi^{ \pm}$cascade travel slower $(v \sim 0.05 c)$ and fill a larger volume, and therefore the elapsed time of the scintillating signal is longer for a $\pi^{ \pm}$and an $e$, shown by the SPACIAL calorimeter to achieve a discrimination of about 100-to- 1 .

The exploitation of these measurements in a collider experiment will depend on many factors, such as channel size, the character of event ensembles, and the fidelity of the measurements themselves. The goal of this alternative detector is to package these capabilities into a comprehensive detector.

$\mu$ separation from $\pi^{ \pm}$We have achieved excellent $\mu-\pi^{ \pm}$separation in the dual readout calorimeter and additional separation using energy balance from the tracker through the calorimeter into the muon spectrometer. A non-radiating $\mu$ has a zero Cerenkov signal in the fiber calorimeter since the Cerenkov angle is larger than the capture cone angle of the 
fiber. The scintillating fibers measure $d E / d x$ of the through-going $\mu$ [16]. Any radiation by the $\mu$ within the body of the calorimeter is sampled equally by the scintillating $(S)$ and Cerenkov $(C)$ fibers [15], and therefore $S-C \approx d E / d x$ independent of the amount of radiation. The distributions of $(S-C)$ vs. $(S+C) / 2$ for $20 \mathrm{GeV}$ and $200 \mathrm{GeV} \pi^{-}$and $\mu^{-}$ are shown in Fig. 8 in which it is evident that for an isolated track the $\pi^{ \pm}$rejection against $\mu$ is about $10^{4}$ at $20 \mathrm{GeV}$ and $10^{5}$ at $200 \mathrm{GeV}$. A further factor of 50 is obtained from the iron-free dual solenoid in which the precisely measured $\mu$ momentum can be matched with the momentum in the central tracker and the radiated energy in the calorimeter. We expect that other effects, such as tracking inefficiencies, will limit this level of rejection before these beam-measured rejections are achieved in practice.

Time-of-flight in CluCou clustertiming and the Ĉerenkov fibers The time history readout of the scintillating and Cerenkov fibers will serve several purposes, viz., $e-\pi$ separation (Fig. 6(b)), neutron measurement for the suppression of fluctuations in binding energy losses, and as a real-time nanosecond monitor of all activity in the volume between the 337-ns bunch crossings, including 'flyers' and beam burps of any kind.

In addition, the sub-ns resolution on the time of arrival of a shower can be used in the time-of-flight of heavy objects such as supersymmetric or technicolor particles that move slowly $(v \sim$ $0.1 c$ ) into the tracking volume, where the cluster-timing is also sub-ns, and decay to light parti-

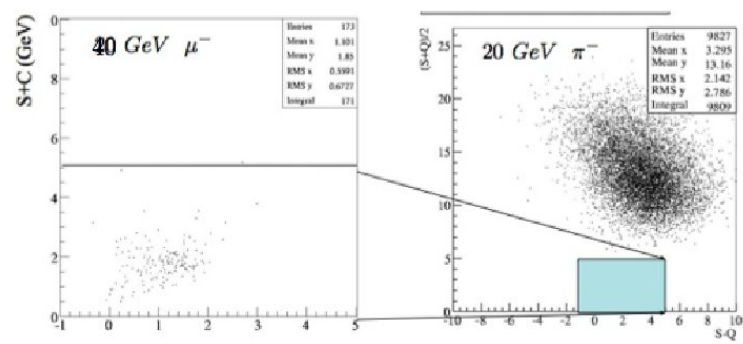

S-C (GeV) $\mathrm{S}-\mathrm{C}(\mathrm{GeV})$

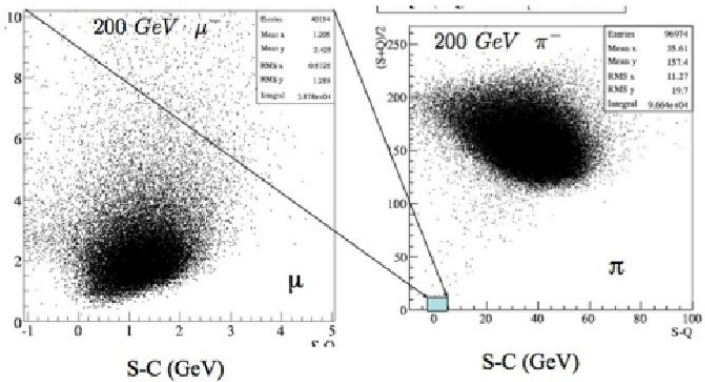

Figure 8: Dual-readout separation of $\mu-\pi^{ \pm}$. cles $(e, \mu, \tau, j$, etc. $)$. Such objects can be easily reconstructed in this detector.

\section{Machine-Detector Interface}

The detector's magnetic field is confined essentially to a cylinder with negligible fringe fields, without the use of iron flux return, Fig. 5 . This scheme offers flexibility in controlling the fields along the beam axis and, in particular, making the fields on the delicate final focus zero, or very close to zero. The twist compensation solenoid just outside the wall of coils is shown in Fig. ??, along with the beam line elements close to the IP. This iron-free configuration [12] allows us to mount all beam line elements on a single support and drastically reduce the effect of vibrations at the final focus (FF), essentially because the beams will coherently move up and down together. In addition, the FF elements can be brought closer to the vertex chamber for better control of the beam crossing. 
The open magnetic geometry of this detector also allows for future physics flexibility for asymmetric energy collisions, the installation of specialized detectors outside the inner solenoid, and magnetic flexibility for non-zero dispersion FF optics at the IP, adiabatic focussing at the IP, and monochromatization of the collisions to achieve a minimum energy spread [12]. Finally, this flexibility and openness allows additions in later years to the detector or to the beam line, and therefore no physics is precluded by this detector concept. A prime example is $\gamma-\gamma$ physics[14], but there are many more exotic possibilities in long-lived dark matter particles, massive slow-moving SUSY particles, etc., that would remain unseen in a conventional detector.

\section{Summary}

This alternative detector contains many new ideas in high energy physics instrumentation that are aimed at a comprehensive detector up to $1 \mathrm{TeV} e^{+} e^{-}$physics at the International Linear Collider. All of the data shown in this paper and all of the performance specifications have already been tested in either beam tests, prototypes, or existing detectors. The difficult problems of incorporating these small successful instruments into a large detector while maintaining the scientific strengths of each present good work in the near future.

\section{References}

[1] International Linear Collider Reference Design Report (2007) Vol. 4, Detectors, August 2007, Eds. Chris Damerell, John Jaros, Akiya Miyamoto, and Ties Behnke.

[2] ILC Tracking R\&D: Report of Review Committee, Beijing, China, 5-8 Feb 2007, (BILCW07), Chris Damerelll (Chair), D. Karlen, W. Lohmann, H. Park, H. Weerts, P. Braun-Munzinger, I. Giomataris, H. Hamagaki, F. Sauli, H. Spieler, M. Tyndel, Y. Unno, C. Yuanbo, O. Qun. J. Brau, J. Haba, B. Zhou 15 April 2007.

[3] ILC Calorimetry R\&D Review, DESY ILC Workshop, 31 May - 4 June 2007, W. Lohmann (Chair).

[4] ILC Vertex Chamber R\&D Review, to be held at the ILC Workshop at Fermilab, October 2007.

[5] 4th Concept Detector Outline Document is available at the WWS-OC website http://physics.uoregon.edu/lc/wwstudy/concepts/.

[6] "Letter of Intent from the Fourth Detector (Ò4thÓ) Collaboration at the International Linear Collider," available at www.4thconcept.org/4LoI.pdf, and appendices on this same website.

[7] "Improving Spatial Resolution and Particle Identification", G.F. Tassielli, F. Grancagnolo, S. Spagnolo, 10th Pisa Meeting on Advanced Detectors, 21-27 May 2006.

[8] "Hadron and Jet Detection with a Dual-Readout Calorimeter", N. Akchurin, et al., Nucl. Instr. Meths. A 537 (2005) 537-561.

[9] item "Neutron Signals for Dual-Readout Calorimetry", Akchurin, N., et al., NIM A598(2009) 422-431; and, ...

[10] "The electromagnetic performance of the RD52 fiber calorimeter," submitted to NIM.

[11] "Particle identification in the longitudinally unsegmented RD52 fiber calorimeter," submitted to NIM.

[12] "A Few Comments on the Status of Detectors for ILC", Alexander Mikhailichenko, CLNS 06/1951, 15 Jan 2006.

[13] "The Muon System of the 4th Concept Detector", F.Grancagnolo, ILC Workshop - ECFA and GLD Joint Meeting, Valencia, Spain, Nov. 5-13, 2006.

[14] V. Telnov, $\gamma-\gamma$ physics.

[15] "Electron Detection with a Dual-Readout Calorimeter", N. Akchurin, et al., Nucl. Instr. Meths. A 536 (2005) 29-51.

Community Summer Study, Snowmass on d'Miss'sip, Minneapolis, MN, USA, 29Jul-9Aug 2013 
[16] "Muon Detection with a Dual-Readout Calorimeter", N. Akchurin, et al., Nucl. Instr. Meths. A 533 (2004) 305-321.

[17] Acosta, D., et al., NIM, A302 (1991) 36. 


\title{
$\mathrm{e}^{+} \mathrm{e}^{-}$Linear Colliders Detector Requirements and Limitations
}

\author{
INPUT TO THE SNOWMASS \\ Instrumentation Frontier WG
}

DRAFT III

$28 /$ August / 2013 
$\mathrm{e}^{+} \mathrm{e}^{-}$Linear Colliders Detector Requirements and Limitations 


\section{Contents}

$\begin{array}{lr}\text { Table of Contents } & 3\end{array}$

$\begin{array}{ll}\text { Introduction } & 7\end{array}$

1 Vertex detector $\quad 11$

1.1 Physics Requirements . . . . . . . . . . . . . . . . . . . . . . . 11

1.2 Detector Requirements . . . . . . . . . . . . . . . . . . 11

1.3 Candidate technologies . . . . . . . . . . . . . . . . . . . 12

1.4 Current and future R\&D efforts . . . . . . . . . . . . . . . . 13

1.5 Summary . . . . . . . . . . . . . . . . . . . 13

2 Silicon Tracking $\quad 15$

2.1 Physics Requirements . . . . . . . . . . . . . . . . . . . . . 15

2.2 Detector Requirements . . . . . . . . . . . . . . . . . . 15

2.3 Candidate Technologies . . . . . . . . . . . . . . . . 16

2.4 Current and Future R\&D Efforts . . . . . . . . . . . . . . . 16

2.5 Summary . . . . . . . . . . . . . . . . . . . . . . . 17

3 TPC tracking $\quad 19$

3.1 Introduction . . . . . . . . . . . . . . . . . . . . . . . 19

3.2 TPC design . . . . . . . . . . . . . . . . . 20

3.3 Status of TPC development . . . . . . . . . . . . . . . 21

3.4 Future R\&D plans . . . . . . . . . . . . . . . . . . 22

4 Silicon-Tungsten Electromagnetic Calorimeter 23

4.1 Physics Requirements . . . . . . . . . . . . . . . . . . . 23

4.2 Detector Requirements . . . . . . . . . . . . . . . . . . . . . . 24

4.3 Candidate technologies . . . . . . . . . . . . . . 25

4.4 Current and future R\&D efforts . . . . . . . . . . . . . . . . . 25

4.5 Summary . . . . . . . . . . . . . . . . . . . 26 
$\mathrm{e}^{+} \mathrm{e}^{-}$Linear Colliders Detector Requirements and Limitations

5 Scintillator-Based Electromagnetic Calorimeter 29

5.1 Physics Requirements . . . . . . . . . . . . . . . . . . . . . . . 29

5.2 Detector Requirements . . . . . . . . . . . . . . . . . . . . . . 29

5.3 Candidate technologies . . . . . . . . . . . . . . . 30

5.4 Current and future R\&D efforts . . . . . . . . . . . . . . . 31

6 Scintillator-Based Hadron Calorimeter 33

6.1 Physics Requirements . . . . . . . . . . . . . . . . . . . . . 33

6.2 Detector Requirements . . . . . . . . . . . . . . . . . . . . . 34

6.3 Candidate technologies . . . . . . . . . . . . . . . . 35

6.4 Current and future R\&D efforts . . . . . . . . . . . . . . . . 35

6.5 Summary . . . . . . . . . . . . . . . . . . 36

$7 \quad$ RPC-based digital hadron calorimeter $\quad 39$

7.1 Physics Requirements . . . . . . . . . . . . . . . . . . . . . . . . . 39

7.2 Detector Requirements . . . . . . . . . . . . . . . . . . . . . 39

7.3 Candidate technologies . . . . . . . . . . . . . . . . . . 40

7.4 Current and future R\&D efforts . . . . . . . . . . . . . . . . . 41

7.5 Summary. . . . . . . . . . . . . . . . . . . . . 41

8 Digital Hadron Calorimetry using GEM 43

8.1 Physics Requirements . . . . . . . . . . . . . . . . . . . . . . 43

8.2 Detector Requirements . . . . . . . . . . . . . . . . . . . . . . 44

8.3 Candidate technologies . . . . . . . . . . . . . . . . . 44

8.4 Current and future R\&D efforts . . . . . . . . . . . . . . . 45

$\begin{array}{llr}9 & \text { Forward calorimetry } & 47\end{array}$

10 Instrumentation for MDI 53

10.1 Physics Requirements . . . . . . . . . . . . . . . . . . 53

10.2 Detector Requirements . . . . . . . . . . . . . . . . . . . . . . . . . . . . . . . . . . . . . .

10.3 Candidate technologies . . . . . . . . . . . . . . . 54

10.4 Current and future R\&D efforts . . . . . . . . . . . . . . . 55

11 Electronics $\quad 57$

11.1 Introduction . . . . . . . . . . . . . . . . . 57

11.2 Current and future R\&D efforts . . . . . . . . . . . . . . 58

11.3 Summary . . . . . . . . . . . . . . . . . 60 
12 Detector Alignment $\quad 61$

12.1 Physics Requirements . . . . . . . . . . . . . . . . . . . . 61

12.2 Detector Requirements . . . . . . . . . . . . . . . . . . . 61

12.3 Candidate technologies . . . . . . . . . . . . . . . . . . . 62

12.4 Current and future R\&D efforts . . . . . . . . . . . . . 63

12.5 Summary . . . . . . . . . . . . . . . . . . . . . 64 
$\mathrm{e}^{+} \mathrm{e}^{-}$Linear Colliders Detector Requirements and Limitations 


\section{Introduction}

\section{Edited by:}

A.P. White University of Texas, Arlington, USA

M. Stanitzki DESY, Germany

The physics program for the International Linear Collider (ILC) [1] places requirements on the technologies for detectors that extend significantly beyond the capabilities of existing collider detectors.

The ILC provides a unique environment due to his beam structure. The ILC bunchtrain of the baseline design contains 1312 bunches, with a spacing of 554 ns and a repetition rate of $5 \mathrm{~Hz}$ [2]. This implies that the detectors need to be "live" for about $1 \mathrm{~ms}$, while the remaining $199 \mathrm{~ms}$ can be used for the read-out or for powering them off (power-pulsing).

The designs of the $\operatorname{SiD}[3,4]$ and ILD $[5,4]$ concepts are the result of many years of creative work by physicists and engineers, backed up by a substantial body of past and ongoing detector research and development. While each component has benefitted from continual development, the concept designs integrate these components into a complete system for excellent measurements of jet energies, based on the Particle Flow Algorithm (PFA) approach, as well as of charged leptons, photons and missing energy.

The vertex systems have been the subject of generic development, exploring new technologies and those proposed for other experiments. It is generally agreed that it is too early to specify a technology choice for each concept as the whole area of research continues to evolve. Significant advances can be expected by the time of the final technology choices. High precision tracking can be achieved either using an all-silicon system as in SiD, or a TPC approach, with external silicon layers, as for ILD.

Calorimetry has been the subject of extensive development both through the CALICE collaboration [6] and independent efforts. The electromagnetic calorimetry solutions focus on fine-grained silicon active layers with tungsten absorber, while the hadron calorimetry has seen the development of a wide 
$\mathrm{e}^{+} \mathrm{e}^{-}$Linear Colliders Detector Requirements and Limitations
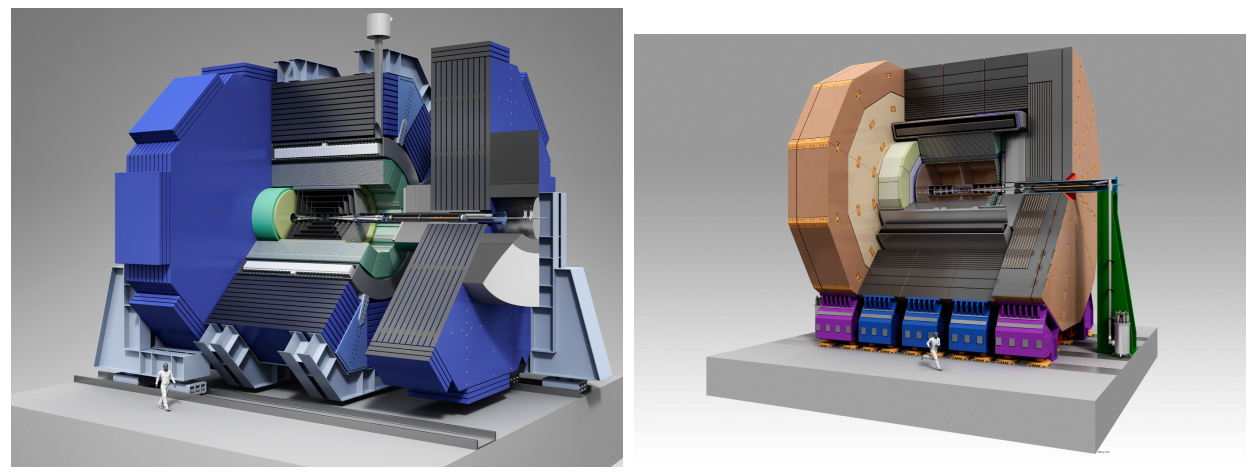

Figure 1: The proposed $\mathrm{SiD}$ (left) and ILD (right) detector concepts for the ILC

variety of active layer solutions coupled with either steel or tungsten absorber layers. Not all technologies are at the same stage of development and work remains to be done to compare the realization of technologies at the level of specific module implementations for each concept. Forward calorimetry has been developed in the context of the FCAL collaboration [7] with solutions proposed for the BeamCAL and LumiCAL systems.

For the muon systems, two active layer technologies are considered - large RPC planes or scintillator strips with embedded wavelength-shifting fibers connected to SiPM's.

For the readout of the various subsystems, a number of solutions have been proposed. For $\mathrm{SiD}$ there has been the general approach of using the KPiX chip for all subsystems except for the vertex and forward calorimeter systems where specific, demanding requirements exist. For ILD there are solutions proposed for the TPC readout and from CALICE for each of the proposed active layer technologies. As for the vertex detector technologies, electronics is an area that can be expected to see significant developments in terms of high channel count chips, reduced format, and reduced power and cooling requirements.

There have also been significant developments in the areas of the MachineDetector Interface (MDI) and alignment. For SiD there has been a preliminary design and prototyping of a Frequency-Scanned Interferometry (FSI) system.

Overall, there has been a considerable amount of development of new detector technologies for the proposed ILC detectors. Some of the techniques have already seen beneficial application to other experiments, such the use of PFA in the CMS experiment [8], and there is significant scope for further applicability in other experiments in the future.

Many of the detector requirements are quite similar for detectors at CLIC [9], 
however the increased levels of machine background and the quite different bunch structure places more stringent requirements on the time-stamping capabilities.

\section{Bibliography}

[1] H. Baer, T. Barklow, K. Fujii, Y. Gao, A. Hoang, S. Kanemura, J. List and H. E. Logan et al., "The International Linear Collider Technical Design Report - Volume 2: Physics,"

[2] T. Behnke, J. E. Brau, B. Foster, J. Fuster, M. Harrison, J. M. Paterson, M. Peskin and M. Stanitzki et al., "The International Linear Collider Technical Design Report - Volume 1: Executive Summary,"

[3] H. Aihara et al. SiD Letter of Intent, 2009, SLAC-R-944.

[4] T. Behnke, J. E. Brau, P. N. Burrows, J. Fuster, M. Peskin, M. Stanitzki, Y. Sugimoto and S. Yamada et al., "The International Linear Collider Technical Design Report - Volume 4: Detectors,"

[5] T. Abe et al. ILD Letter of Intent, 2009,, arXiv:1006.3396 [hep-ex].

[6] CALICE Collaboration https://twiki.cern.ch/twiki/bin/view/ CALICE/CaliceCollaboration

[7] FCAL Collaboration http://fcal.desy.de/

[8] [CMS Collaboration], "Particle-Flow Event Reconstruction in CMS and Performance for Jets, Taus, and MET," CMS-PAS-PFT-09-001.

[9] L. Linssen, A. Miyamoto, M. Stanitzki and H. Weerts, "Physics and Detectors at CLIC: CLIC Conceptual Design Report," 
$\mathrm{e}^{+} \mathrm{e}^{-}$Linear Colliders Detector Requirements and Limitations 


\section{Chapter 1}

\section{Vertex detector}

Edited by:

Y. Sugimoto KEK, Japan

\subsection{Physics Requirements}

The identification of heavy (charm and bottom) quarks and tau leptons is essential for the ILC physics programme. Such "flavor tagging" is performed by reconstructing decay vertices of short lived particles, such as $D$ or $B$ mesons, and a precise vertex detector plays an essential role. The performance of a vertex detection system is often expressed by the impact parameter resolution of charged particles. The performance goal of the ILC vertexing system we set is a resolution on the track impact parameter of $\sigma_{b}<5 \oplus 10 / p \beta \sin ^{3 / 2} \theta \mu \mathrm{m}$.

\subsection{Detector Requirements}

In order to reach such a high performance level, the ILC vertex detectors should comply with the specifications shown in Table 1.1 .

The requirement for the spatial resolution and the material budget for $\operatorname{SiD}[1,2]$ are less severe than for ILD $[2,3]$ because the distance between the interaction point and the first layer is less than ILD. On the other hand, the requirement for the readout speed is more severe for $\mathrm{SiD}$ than ILD. The tracking system of $\mathrm{SiD}$ consists of five layers of the vertex detector and five layers of silicon strip detectors. In order to obtain high tracking efficiency under the high background hit rate due to "pair backgrounds", time stamping capability which can separate each beam bunch and keep the pixel occupancy very low is required. In case of ILD, the track finding is achievable by outer trackers (TPC and silicon 
$\mathrm{e}^{+} \mathrm{e}^{-}$Linear Colliders Detector Requirements and Limitations

\begin{tabular}{lcc}
\hline & SiD & ILD \\
\hline Spatial resolution of the 1st layer $(\mu \mathrm{m})$ & 5 & 3 \\
Radius of the $1^{\text {st }}$ layer $(\mathrm{mm})$ & 14 & 16 \\
Material budget $\left(\% \mathrm{X}_{0} /\right.$ layer $)$ & 0.3 & 0.15 \\
Time stamping $(\mu \mathrm{s})$ & $0.3-0.7$ & $10-1000$ \\
\hline
\end{tabular}

Table 1.1: Specifications of vertex detectors for SiD $[1,2]$ and ILD $[2,3]$.

inner tracker). Therefore, the pixel occupancy of 2 or $3 \%$ is acceptable, and much less time stamping capability is required for the ILD vertex detector.

The power consumption should be low enough to minimize the material budget of the cooling system inside the detector sensitive volume. Power savings may be obtained by exploiting the beam time structure and power pulse the sensors equipping the detector. Alternatively, the signals may be integrated over the complete bunch train and read out in between consecutive trains at very low frequency.

The required radiation tolerance follows entirely from the beam related background (i.e. beamstrahlung) which is expected to affect predominantly the innermost layer. The tolerance required amounts to about $1 \mathrm{kGy}$ and a neutron flux of $10^{11} \mathrm{n}_{e q} / \mathrm{cm}^{2}$ per annum, including some safety margin.

\subsection{Candidate technologies}

Since the early stage of the ILC detector R\&D, a lot of sensor technologies have been proposed and studied. Those technologies are

- Column parallel CCDs (CPCCDs) [4]

- Fine pixel CCDs (FPCCDs) [5]

- CMOS monolithic active pixel sensors [6]

- Depleted FET sensors (DEPFETs) [7]

- Chronopixel sensors [8]

- 3D vertically integrated pixel sensors (VIP) [9]

- Hybrid pixels 
- In-situ storage image sensors (ISIS) [10]

For some of them, the R\&D activity has been inactive due to technical and/or financial problems.

\subsection{Current and future R\&D efforts}

For ILD, R\&D efforts for CMOS pixel sensors, FPCCDs and DEPFETs, are actively being carried out as candidates for the baseline sensor technology. For SiD, chronopixel sensors, VIP 3D chips, and thinned hybrid detectors with highdensity interconnect are being developed. Most of the sensor technologies have been demonstrated to work at least with small prototypes. However, there is still room for a lot of $R \& D$ in order to get ready for construction.

In addition to the sensor technologies, R\&D efforts on the ultra-light support structure are also going on. The power delivery scheme to minimize the power consumption and the cooling system are also important R\&D issues currently pursued.

Because vertex detectors of ILC detectors are relatively easily replaceable, the evolution of sensor technologies can be exploited quite efficiently, in particular, to comply with the manyfold increase of the beam related background expected at $1 \mathrm{TeV}$ collision. Therefore, future $R \& D$ for the detector upgrade should be continued. Much shorter readout time for ILD is one of the R\&D issues to be pursued in future.

\subsection{Summary}

Unprecedented high performance is required for the vertex detectors at ILC. Many groups are involved in the R\&D effort for the ILC vertex detectors. Some sensor technologies have reached proof-of-principle stage. However, the maturity of the R\&D is still far from the level of construction of a real vertex detector. More R\&D efforts including mechanical aspects are absolutely necessary.

\section{Bibliography}

[1] H. Aihara et al. SiD Letter of Intent, 2009, SLAC-R-944.

[2] T. Behnke, J. E. Brau, P. N. Burrows, J. Fuster, M. Peskin, M. Stanitzki, Y. Sugimoto and S. Yamada et al., "The International Linear Collider Technical Design Report - Volume 4: Detectors," 
$\mathrm{e}^{+} \mathrm{e}^{-}$Linear Colliders Detector Requirements and Limitations

[3] T. Abe et al. ILD Letter of Intent, 2009,, arXiv:1006.3396 [hep-ex].

[4] K. D. Stefanov, "CCD-based vertex detector for the future linear collider," Nucl. Instrum. Meth. A 549 (2005) 93.

[5] Y. Sugimoto, H. Ikeda, D. Kamai, E. Kato, A. Miyamoto, H. Sato and H. Yamamoto, "\& Status of FPCCD Vertex Detector for ILD," arXiv:1202.5832 [physics.ins-det].

[6] R. Turchetta, J. D. Berst, B. Casadei, G. Claus, C. Colledani, W. Dulinski, Y. Hu and D. Husson et al., "A monolithic active pixel sensor for charged particle tracking and imaging using standard VLSI CMOS technology," Nucl. Instrum. Meth. A 458 (2001) 677.

[7] O. Alonso et al. [DEPFET Collaboration], "DEPFET active pixel detectors for a future linear $e^{+} e^{-}$collider," arXiv:1212.2160 [physics.ins-det].

[8] C. Baltay, W. Emmet, D. Rabinowitz, J. Brau, N. Sinev and D. Strom, "Chronopixel Vertex Detectors for Future Linear Colliders," arXiv:1109.2811 [physics.ins-det].

[9] G. Deptuch et al. [ASIC Development Group Collaboration], "A vertically integrated pixel readout device for the Vertex Detector at the International Linear Collider," IEEE Trans. Nucl. Sci. 57 (2010) 880.

[10] C. Damerell, Z. Zhang, R. Gao, J. J. John, Y. Li, A. Nomerotski, A. Holland and G. Seabroke et al., "First results with prototype ISIS devices for ILC vertex detector," Nucl. Instrum. Meth. A 624 (2010) 465. 


\section{Chapter 2}

\section{Silicon Tracking}

Edited by:

T.K. Nelson

SLAC, USA

\subsection{Physics Requirements}

Since particle flow calorimetry depends upon efficient and precise reconstruction of charged particles, tracking is a cornerstone of most physics analyses at an $\mathrm{e}^{+} \mathrm{e}^{-}$collider. However, certain critical measurements place special emphasis on the acceptance and precision of the tracking system. For the study of Higgs decays in Higgs-strahlung events, the precision with which the recoiling $Z$ is reconstructed from high-momentum tracks determines the background rate and therefore the precision of Higgs coupling measurements. Furthermore, for Higgs decay to muons, the low expected rate and narrow intrinsic width require extraordinary resolution of the high-momentum muons for signal observation. Meanwhile, decays of supersymmetric particles with small mass splittings require excellent low-momentum tracking including good forward coverage to precisely determine endpoint energies and untangle the spectroscopy of states.

\subsection{Detector Requirements}

In current detector concepts, the outer silicon tracker is part of an integrated system where pattern recognition and track reconstruction include the vertex detector and a highly granular electromagnetic calorimeter: the outer silicon tracker has a primary role only in measuring transverse momentum. High- 
$\mathrm{e}^{+} \mathrm{e}^{-}$Linear Colliders Detector Requirements and Limitations

momentum resolution scales linearly with single-hit $r-\phi$ resolution and with the square root of the number of $r-\phi$ measurements. However, since material in a silicon tracker also scales with the number of measurement planes, extraneous measurements penalize low-momentum tracking, where even aggressive material budgets limit performance for tracks with transverse momentum below $\sim 30$ $50 \mathrm{GeV}$. These facts motivate an outer silicon tracker with the best possible $r-\phi$ resolution, the smallest possible material per measurement plane, and the smallest number of hits that allow for robust track reconstruction. Together with excellent forward coverage, timing resolution that minimizes backgrounds, and reasonable cost and complexity, these are the key attributes of silicon tracking for an $\mathrm{e}^{+} \mathrm{e}^{-}$collider.

\subsection{Candidate Technologies}

Silicon sensors with granular readout in $r-\phi(\sim 50 \mu \mathrm{m}$ or finer $)$ easily provide the required hit resolution. Using the largest readout granularity in $z$ that provides robust pattern recognition minimizes the material from readout, power and cooling. The simplest realization of these principles are designs that utilize silicon microstrip sensors. In order to minimize material, front end readout uses pulsed power to reduce power consumption and cooling requirements by a factor of 100 and such designs are air cooled. To deal with large peak currents with a limited mass of conductor, DC-DC conversion and local charge storage are planned. These features also reduce the likelihood of significant vibrations from periodic Lorentz forces on power conductors. Fully integrated readout chips can be bonded directly to the surface of sensors, eliminating the mass of hybrid circuit boards. Tagging the time of buffered hits during the bunch train, with digitization and readout of hits between trains, reduces digital noise on the front ends and eliminates out-of-time background hits during track reconstruction. Such microstrip trackers have demonstrated acceptable tolerance to backgrounds for proposed $\mathrm{e}^{+} \mathrm{e}^{-}$colliders. However, sensors with pixellated readout similar to those proposed for the vertex detectors would make tracking even more robust in dense jets at the cost of additional system complexity, technological risk, and challenges in low-mass cooling and power.

\subsection{Current and Future R\&D Efforts}

The current R\&D efforts are focused on readout electronics, powering and support for a large microstrip-based silicon tracker. The first of these efforts, undertaken by the SiD detector concept $[1,2]$, focuses on the development 
of the $\mathrm{KPiX}$ [3] readout $\mathrm{ASIC}$, designed to be bump-bonded to the face of a double-metal readout sensor. Capable of storing four time-stamped hits per bunch train, this power-pulsed chip provides single ILC-bunch timing in a power envelope that allows gas cooling and should scale to time resolution of $10 \mathrm{~ns}$ for colliders with smaller bunch spacings. The SiD tracker envisions single-sensor tiles covering a set of nested, cylindrical carbon fiber supports for the stiffest structure with the least possible material: less than $1 \% \mathrm{X}_{0}$ /layer. The modules in the barrel region are single-sided while the forward modules, mounted on conical disks that close the cylinders, are double sided with a stereo angle to assist in forward tracking. The SiLC collaboration [4] is developing a similar concept for tracking layers that surround the central TPC and which comprise the forward tracking system of the proposed ILD $[2,5]$ detector.

\subsection{Summary}

Pure and efficient tracking over the full solid angle of the detector, and with excellent resolution for both high and low-momentum tracks, is critical to physics at future $\mathrm{e}^{+} \mathrm{e}^{-}$colliders. Concepts utilizing silicon microstrip sensors with low-power readout, gas cooling, and low mass support are able to meet these requirements. If the future, thinner and more granular trackers based upon monolithic active pixel sensors may become feasible that would outperform microstrip trackers in dense jets encountered at the highest energies envisioned for such experiments.

\section{Bibliography}

[1] H. Aihara et al. SiD Letter of Intent, 2009, SLAC-R-944.

[2] T. Behnke, J. E. Brau, P. N. Burrows, J. Fuster, M. Peskin, M. Stanitzki, Y. Sugimoto and S. Yamada et al., "The International Linear Collider Technical Design Report - Volume 4: Detectors,"

[3] D. Freytag, R. Herbst, J. Brau, M. Breidenbach, R. Frey, G. Haller, B. Holbrook and R. Lander et al., "KPiX, An Array of Self Triggered Charge Sensitive Cells Generating Digital Time and Amplitude Information," SLACPUB-13462.

[4] A. Savoy-Navarro, "Overview of the SiLC R and D Activities," arXiv:0903.4361 [physics.ins-det].

[5] T. Abe et al. ILD Letter of Intent, 2009,, arXiv:1006.3396 [hep-ex]. 
$\mathrm{e}^{+} \mathrm{e}^{-}$Linear Colliders Detector Requirements and Limitations 


\section{Chapter 3}

\section{TPC tracking}

Edited by:

T. Behnke

DESY, Germany

\subsection{Introduction}

A time projection chamber is one of several proposals for a central tracker at a future linear collider. It is part of the ILD $[1,2]$ proposal at the ILC, but it is also investigated for use at CLIC [3]. A time projection chamber (TPC) has a number of distinct properties. A TPC provides a large number of measured spatial coordinates along a track. The measurements are truly three dimensional. The TPC can instrument a large volume while introducing small amounts of dead material. It promises to deliver a very homogeneous coverage, with small insensitive areas. By immersing the complete TPC in a magnetic field which points in the same direction as the electric field the diffusion during the rather long drift distance for the electrons towards the readout endplates can be controlled. For the ILD detector a central magnetic field of $3.5 \mathrm{~T}$ is proposed. A spatial resolution below $100 \mu \mathrm{m}$ in r-phi, better than one $\mathrm{mm}$ in $\mathrm{z}$, is possible in such a configuration, In addition to good spatial resolution a TPC offers the possibility to use the specific energy loss of a charged particle in the gas $(d E d x)$ to help in the identification of the particle type. The ILD TPC is going to be operated at room temperature and nominal pressure, which somewhat limits the achievable $\mathrm{dE} / \mathrm{dx}$ precision. Still a separation between electrons and pions should be possible by $\mathrm{dE} / \mathrm{dx}$ at the level of two sigma for nearly all relevant momenta. The detector at the linear collider should be operated without a dedicated hardware trigger. This puts stringent requirements on the capabilities 
$\mathrm{e}^{+} \mathrm{e}^{-}$Linear Colliders Detector Requirements and Limitations

\begin{tabular}{ll}
\hline Parameter & Performance goal \\
\hline$\sigma(r-\phi)$ & $100 \mu \mathrm{m}$ fur full drift \\
$\sigma(z)$ & $0.4-1.4 \mathrm{~mm}$ \\
Two-Hit resolution & $2 \mathrm{~mm}(r-\phi), 6 \mathrm{~mm}(\mathrm{z})$ \\
Momentum resolution & $\delta\left(1 / p_{t}\right)=10^{-4} / \mathrm{GeV} / \mathrm{c}$ (TPC only) \\
$d E / d x$ resolution & $5 \%$ \\
\hline
\end{tabular}

Table 3.1: Main performance parameters of a TPC at a linear collider

of the detectors to be operated continuously throughout one bunch train of the collider. The TPC can do this well, and can, as long as the overall occupancy is kept at a reasonable level, provide precision measurements for the long bunch trains anticipated in the ILC. These strong points for a TPC are to some extend offset by limited spatial resolution possible in a gaseous detector, compared to semi-conductor detector. However the large number of individual measurement point, larger by at least one order of magnitude, more than compensates this. In addition the large number of points is well suited for a detector where the topological reconstruction of events as it is done in the context of particle flow is of prime importance. Over the past ten years an internationally coordinated $R \& D$ effort has been undertaken to demonstrate the feasibility of using a TPC at a linear collider detector. Coordinated by the LC-TPC collaboration this effort has led to the design and construction of several prototypes, testing several different candidate technologies.

\subsection{TPC design}

The design of a TPC for a linear collider is challenging in several ways. The TPC will be large, around $4 \mathrm{~m}$ in diameter, and about $5 \mathrm{~m}$ in total length, should represent a small amount of insensitive material, and be readout out with high speed and high reliability from typically two endplates situated at the two opposite ends of the detector. The main performance criteria are summarized in Table 3.1

The field cage, which is the main structural element of the detector, is realized as a light weight cylinder built from composite materials. On the inside surface of the cylinder field shaping strips provide the proper grading of the potential inside the drift volume. In the center a membrane acts as cathode, at the two ends a light weight endplate closes the gas volume and provides support for readout modules. 


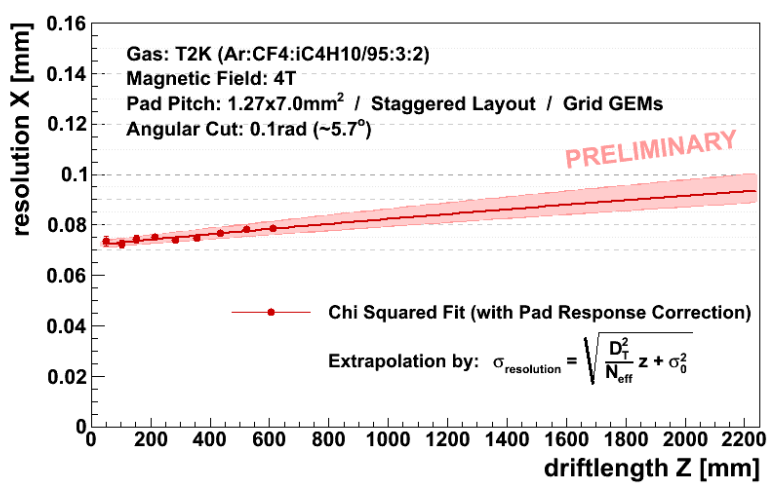

Figure 3.1: Measured point resolution in a GEM TPC at a field of 4T. The measured points are extrapolated to cover the complete drift distance as expected for the full size TPC.

The gas amplification will be done with micro pattern gas detectors, MPGD. Two technologies, gas electron multipliers, or Micromegas, are under consideration.

\subsection{Status of TPC development}

Following a series small scale proof-of-principle experiments a number of experiments have been done to develop and demonstrate the TPC system as proposed for the LC. To this end a large field cage has been constructed, which can be operated with different readout technologies inside a magnetic field. This facility has been used with GEM readout modules, micromegas readout modules, and with a novel combination of gas amplification and silicon pixel detectors (PixelTPC readout). Both GEM and micromegas readout modules could demonstrate the required spatial resolution. In Figure 3.1 the measured point resolution is shown obtained with a GEM module, at a field of $4 \mathrm{~T}$. Very similar results have been obtained with a micromegas based module.

With the current experimental setup the measurement of the momentum resolution is difficult. On the one hand side the magnetic field shows large inhomogeneity, which will need to be corrected. Work on this has just started. On the other hand the ultimate momentum resolution measurement can only be done once the setup is extended to include a high precision external device to provide an independent determination of the momentum of the beam particle. 
$\mathrm{e}^{+} \mathrm{e}^{-}$Linear Colliders Detector Requirements and Limitations

A significant challenge remains the mechanical design of the system. In particular the complex endplate will introduce material into the detector, and efforts are under way to minimize this. This requires significant progress in the miniaturization of the readout electronics, and advanced construction techniques for the endplate itself.

\subsection{Future R\&D plans}

The study of a TPC for the linear collider has made considerable progress since it started about ten years ago. The basic requirements for a TPC have been demonstrated, and the feasibility of the technologies has been shown. A large prototype has been constructed and operated in beams with different technologies.

Over the next years the setup will be improved to allow an unbiased study of the moment resolution. Engineering studies will be done to improve the endplates, to develop a light weight version, and to integrate more closely the readout with the endplate. The final test, namely the measurement of all resolutions including the $\mathrm{dEdx}$ one, will require that the complete setup be re-located from the current electron only test beam facility at DESY to a hadron beam, either at CERN or at FNAL. However already now the fundamental feasibility of a MPGD TPC has been demonstrated and is now an accepted and central option for a tracker at the linear collider.

\section{Bibliography}

[1] T. Behnke, J. E. Brau, P. N. Burrows, J. Fuster, M. Peskin, M. Stanitzki, Y. Sugimoto and S. Yamada et al., "The International Linear Collider Technical Design Report - Volume 4: Detectors,"

[2] T. Abe et al. ILD Letter of Intent, 2009,, arXiv:1006.3396 [hep-ex].

[3] L. Linssen, A. Miyamoto, M. Stanitzki and H. Weerts, "Physics and Detectors at CLIC: CLIC Conceptual Design Report,"

[4] LCTPC Collaboration http://www. Ictpc.org/ 


\title{
Chapter 4
}

\section{Silicon-Tungsten Electromagnetic Calorimeter}

\author{
Edited by: \\ R. Frey \\ Department of Physics and Center for High-Energy Physics, \\ University of Oregon
}

\subsection{Physics Requirements}

In order to provide unique and complementary information on the nature of highenergy events, it should be required of Linear Collider (LC) detectors that they provide excellent reconstruction of hadronic final states in multi-jet topologies. One statement for a requirement on jet reconstruction is that intermediate particles which decay into jets, such as W, Z, or top, can be identified and isolated. This places unprecedented requirements on 2-jet or 3-jet mass resolution, typically at the level of a few $\%$ using the particle-flow (PFA) technique. This makes challenging demands on electromagnetic calorimeters (ECAL). The most critical PFA-driven requirements are fine segmentation and high density to limit the lateral size of electromagnetic showers. Together these provide the crucial PFA ingredient of resolution of individual particles in jets. Such a dense, highly segmented ECAL can be thought of as an imaging calorimeter. In addition to aiding in excellent reconstruction of jets, an imaging ECAL will similarly allow identification of different hadronic tau decay modes, which could be crucial for measurements of tau polarization. The imaging requirements will allow some additional capabilities: The ECAL can become an element of the tracking system, providing, for example, the reconstruction of neutrals which decay in the outer 
$\mathrm{e}^{+} \mathrm{e}^{-}$Linear Colliders Detector Requirements and Limitations

layers of the central tracker. Finally, dense photon-initiated showers can be reconstructed and "vertexed" to determine if they originate from the primary interaction point or result from the decay of an (exotic) long-lived particle at a displaced vertex.

The above requirements are the primary physics drivers of ECAL designs for future linear colliders. However, the following more typical requirements most also be met:

- Measurement of beam-energy electrons and positrons (and photons) from (radiative) Bhabha scattering. This provides sensitivity to contact terms from new physics, the angular distribution provides important information on electroweak couplings, and the acollinearity distributions provides a key piece of the measurement of the luminosity spectrum of collision energies.

- Identification of electrons from $Q \rightarrow Q^{\prime} e \nu$

In summary, segmentation and density are the primary design requirements. No physics requirement has been identified which requires electromagnetic energy resolution, parameterized as $b / \sqrt{E}$, better than $b$ in the range 0.15 to 0.20 .

\subsection{Detector Requirements}

A sampling calorimeter with highly segmented readout layers will meet the basic requirements with moderate energy resolution. A dense radiator such as tungsten provides a small Molière radius. If the readout layers are kept thin, a small effective Molière radius can be maintained. Simulations studies have shown that the ECAL pixel size should be roughly in the range 10 to $20 \mathrm{~mm}^{2}$ or less, depending on the proximity of the ECAL to the interaction point. Meanwhile, dense showers can deposit the equivalent of $\sim 10^{3}$ MIPs in a single pixel near shower maximum. Since single MIPs must also be well measured, the readout must therefore be linear over a dynamic range of $10^{3}$ or more. The above requirements imply that the readout electronics necessarily be integrated with the active detector elements.

The timing structure of an linear collider plays an important role in the ECAL design in several ways. Firstly, the beam crossings are organized into beam trains. The trains are widely separated in time. The time is sufficient to allow the power to the readout chips to be reduced between trains. The fraction of time during trains compared to the total time depends on the LC technology, but is typically $1 \%$. The greatly reduced heat load due to this power pulsing capability thus makes it possible to cool the ECAL entirely passively. This can greatly simplify the designs, eliminate the inert material associated with active cooling, 
and preserve the density of the detector. In addition, the timing and number of beam crossings within a bunch train determine the amount of background which is integrated within a readout frame of the central trackers. This is especially an issue for the "warm" LC technologies, such as CLIC [1]. Having a timing capability at the level of $\sim 1$ ns for charged tracks entering the ECAL can aid in associating crossings with physics collisions to their proper location in the readout sequence.

\subsection{Candidate technologies}

The requirements point to a sampling calorimeter with dense radiator layers and thin, highly segmented readout layers. Tungsten is a good candidate for the radiator. It is dense, has a small Molière radius, and, depending on the alloy, can be manageably formed into adequately flat plates of large area.

The leading candidate for the active layers is silicon sensors which are pixellated. This choice has several advantages. Silicon sensors can be arbitrarily segmented within the broad range of possible requirements. The readout layers employing silicon can be made very thin, perhaps as thin as $1 \mathrm{~mm}$ or less. Integrating the readout chips to the sensors is tractable in terms of current technologies. In this approach, each readout chip would read out hundreds to thousands of pixels.

Other technologies have been considered which take on a modified approach. For example, if one can make the pixels sufficiently small, then the limited dynamic range may make it possible to employ a simplified readout, perhaps with one readout per pixel. Such an approach has been taken using the MAPS technology for combining the active elements with readout in single integrated pixels.

\subsection{Current and future R\&D efforts}

The current $R \& D$ is centered on the detector concepts being considered for the ILC [2] and CLIC [1], all of which employ silicon-tungsten as a leading ECAL technology. The R\&D has taken different approaches by the groups involved. The silicon-tungsten effort within the CALICE [3] collaboration has been carried out by building and testing a series of prototype modules, the first of which did not include integrated electronics, but was thoroughly tested in beams at DESY, CERN, and Fermilab. They have more recently been designing and fabricating a prototype module which does include integrated electronics. The silicon-tungsten effort by the $\operatorname{SiD}[5,6]$ group has focused entirely on a highly 
$\mathrm{e}^{+} \mathrm{e}^{-}$Linear Colliders Detector Requirements and Limitations

integrated readout approach using the $\mathrm{KPiX}$ [7] chip. This group is currently fabricating a prototype module to be tested in beam at SLAC.

Future R\&D could reasonably focus on several important areas. First, the prototypes resulting from the current $R \& D$ needs to be completed by completing the prototype modules and fully characterizing their performance. Second, the technologies to provide the necessarily high level of integration need to be improved. Part of this will be to improve and make more reliable the interconnect technologies, especially for chip to sensor. Finally, new approaches for the segmented active layers, such as Monolithic Active Pixel Sensors (MAPS), can be pursued.

\subsection{Summary}

The demands from LC physics, driven by the requirement to accurately reconstruct multi-jet final states [4], can be met by dense, imaging calorimeters. The leading implementations are sampling calorimeters with alternating layers of tungsten and segmented silicon with integrated readout. Completing the current $R \& D$ will leave this technology in good shape for a next round of $R \& D$ prior to realization at a future $\mathrm{LC}$.

\section{Bibliography}

[1] L. Linssen, A. Miyamoto, M. Stanitzki and H. Weerts, "Physics and Detectors at CLIC: CLIC Conceptual Design Report,"

[2] T. Behnke, J. E. Brau, B. Foster, J. Fuster, M. Harrison, J. M. Paterson, M. Peskin and M. Stanitzki et al., "The International Linear Collider Technical Design Report - Volume 1: Executive Summary,"

[3] CALICE Collaboration https://twiki.cern.ch/twiki/bin/view/ CALICE/CaliceCollaboration

[4] H. Baer, T. Barklow, K. Fujii, Y. Gao, A. Hoang, S. Kanemura, J. List and H. E. Logan et al., "The International Linear Collider Technical Design Report - Volume 2: Physics,"

[5] H. Aihara et al. SiD Letter of Intent, 2009, SLAC-R-944.

[6] T. Behnke, J. E. Brau, P. N. Burrows, J. Fuster, M. Peskin, M. Stanitzki, Y. Sugimoto and S. Yamada et al., "The International Linear Collider Technical Design Report - Volume 4: Detectors," 
[7] D. Freytag, R. Herbst, J. Brau, M. Breidenbach, R. Frey, G. Haller, B. Holbrook and R. Lander et al., "KPiX, An Array of Self Triggered Charge Sensitive Cells Generating Digital Time and Amplitude Information," SLACPUB-13462. 
$\mathrm{e}^{+} \mathrm{e}^{-}$Linear Colliders Detector Requirements and Limitations 


\section{Chapter 5}

\section{Scintillator-Based Electromagnetic Calorimeter}

Edited by:

T. Takeshita

Shinsu University, Japan

\subsection{Physics Requirements}

In order to measure Higgs self-couplings for example, two Higgs final states with four b-jets have to be separated clearly from $\mathrm{WW} / \mathrm{ZZ}$ final states in addition to $\mathrm{HH}$, and there exists another $\mathrm{Z}$ from the Higgstrahlung process. In total, there are six jets which contains at least four b-jets at the ILC at $350 \mathrm{GeV}$. Such a multi-jet environment is typical of ILC physics and the jets have to be identified by measuring the jet energy precisely.

\subsection{Detector Requirements}

There are two physics issues that impose requirements on the calorimeter. One is jet energy resolution to separate $\mathrm{W} / \mathrm{Z} /$ Higgs. This is not a specific physics process, however, it is a component of any process which includes jet final states. The other is the $\tau$ jet separation and reconstruction.

The jet energy resolution is the first requirement. Since the PFA is the driving force for event reconstruction, which includes the calorimeters, the performance of the PFA drives calorimeter parameters, such as segmentation, both longitudinal and transverse. The electromagnetic (EM) calorimeter is required to have a 
$\mathrm{e}^{+} \mathrm{e}^{-}$Linear Colliders Detector Requirements and Limitations

small granularity of $5 \mathrm{~mm}$, according to Pandora PFA results, in order to have good separation of jets originating from either $\mathrm{W}$ or $\mathrm{Z}$ decays.

The second issue is $\tau$ jet reconstruction which also requires small segmentation for the reconstruction of photons and neutral pions in tau decay particles, to separate the decay branches. The current requirement is $5 \mathrm{~mm}$, again from ILD-LOI [1].

\subsection{Candidate technologies}

Here a scintillator based Electromagnetic CALorimeer (ECAL) is discussed.

In order to fulfill the PFA requirements, the ECAL has to be fine segmented not only transversely on the surface of the detector with $5 \mathrm{~mm} \times 5 \mathrm{~mm}$ cells, but also in the longitudinal direction. This means fine segmentation in three dimensions is demanded. In order to minimize the EM shower size in the lateral direction, the absorber is chosen to be tungsten. The total thickness of tungsten is determined to have enough stopping power for $250 \mathrm{GeV}$ electrons from Bhabha events in the ECAL alone. This results about $25 \mathrm{X}_{0}$ (radiation length) or $88 \mathrm{~mm}$.

A scintillator solution for the PFA ECAL should be as thin as possible. This is one of the challenges of the detector R\&D. The current solution is a scintillator thickness between $1 \mathrm{~mm}$ and $2 \mathrm{~mm}$, with a thin photo-sensor attached. The combination of the scintillator and photo-sensor is the key issue for the ECAL. Since minimal ionizing particles (MIPS) have to be identified in each layer and the $250 \mathrm{GeV}$ electrons should be also measured, the detection unit has to have a huge dynamic range, even with fine segmentation. From a simulation of MIPS and Bhabha events, we have to keep the dynamic range from 1 MIP to 3500 MIPS maximum energy deposit in a unit. Recently we have successfully developed a semiconductor photo-sensor of very thin and small sizes for the scintillator calorimeter, which has $1 \mathrm{~mm} \times 1 \mathrm{~mm}$ acceptance of photons and the ability to count the number of photons. Development has progressed to reach a level of 2500 MIPS.

Maximum segmentation would be $5 \mathrm{~mm} \times 5 \mathrm{~mm}$ for a scintillator ECAL, however, that would cost too much. Thus we introduce a scintillator strip of $5 \mathrm{~mm} \times 45 \mathrm{~mm}$ for example, in order to reduce the cost, maintaining the PFA performance as far possible. This will be achieved by introducing a novel idea of a Strip Splitting Algorithms (SSA). Degradation of PFA performance is small enough as shown in the Figure.

By introducing long scintillator strips, we are able to reduce the cost of the detector to be one tenth compared to other designs. 


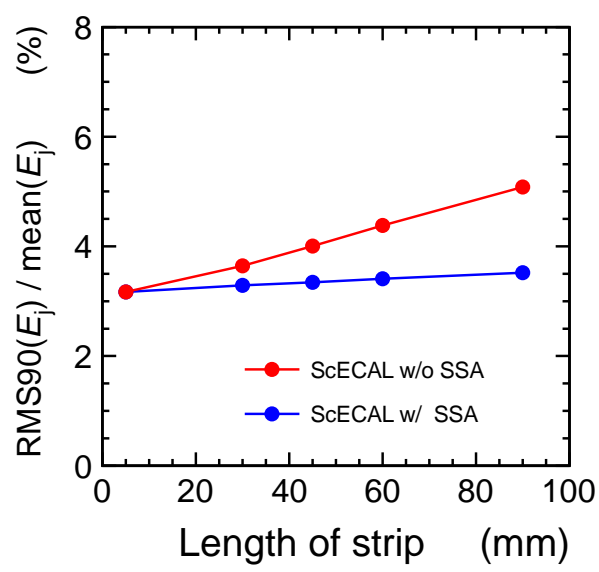

Figure 5.1: Jet energy resolution for $e^{+} e^{-} \rightarrow Z \rightarrow u \bar{u}, d \bar{d}, s \bar{s}$ event at $200 \mathrm{GeV}$ centre of mass energy as a function of strip length, for the ScECAL option, shown without (red) and with (blue) the strip splitting algorithm [2].

\subsection{Current and future R\&D efforts}

1. A scintillator strip ECAL module has been constructed and tested in electron beams and gave good results as shown in the figure. The ECAL consists of 2160 channels of scintillator strips in total, composed of 72 channels/layer and 30 layers in the detector. The scintillator strip in this case is $10 \mathrm{~mm} \times 45 \mathrm{~mm} \times 3 \mathrm{~mm}$ for the test prototype.

2. Scintillator ECAL prototype has been tested at the Fermilab MT6 beam line.

3. Smaller strips of $5 \mathrm{~mm} \times 45 \mathrm{~mm} \times 2 \mathrm{~mm}$ have been tested on the bench and verified to have good uniformity and enough photo-electrons/MIP.

4. Scintillator strip homogeneity along the center line.

5. The photo-sensor has non-linearity, because of the number of pixels in the sensitive area, and thus increasing the number of pixels will expand the linearity. Current test results are given by 1600 pixels $/ \mathrm{mm}^{2}$, and further improvement to 10000 pixels $/ \mathrm{mm}^{2}$ is already achieved.

6. There is still some room for more improvement. The photo-sensor is occupying $2 \%$ of the surface of the ECAL. To reduce the dead surface by setting the photo-sensor behind the scintillator is now under study. 
$\mathrm{e}^{+} \mathrm{e}^{-}$Linear Colliders Detector Requirements and Limitations

\section{Bibliography}

[1] T. Abe et al. ILD Letter of Intent, 2009,, arXiv:1006.3396 [hep-ex].

[2] T. Behnke, J. E. Brau, P. N. Burrows, J. Fuster, M. Peskin, M. Stanitzki, Y. Sugimoto and S. Yamada et al., "The International Linear Collider Technical Design Report - Volume 4: Detectors," 


\title{
Chapter 6
}

\section{Scintillator-Based Hadron Calorimeter}

\author{
Edited by: \\ F. Sefkow \\ DESY, Germany
}

\subsection{Physics Requirements}

The calorimeter system of a future linear collider detector is optimized for best jet energy resolution. The goal is to identify heavy bosons $-\mathrm{W}, \mathrm{Z}$ and $\mathrm{H}-$ on the basis of the invariant mass in hadronic final states. The mass resolution should thus be of the order of $3 \mathrm{GeV}$ at the $\mathrm{W}$ or $\mathrm{Z}$ mass, which translates into a requirement for the jet energy resolution of about 3-4\%.

A physics channel particularly sensitive to the jet energy performance is obviously the WW final state, to be separated from its irreducible ZZ background. Other examples are the measurement of the $\mathrm{H} \rightarrow \mathrm{WW}^{*}$ branching ratio, which together with the cross section for Higgs production in the $W$ fusion channel enters into the determination of the Higgs total width, or the measurement of the triple-Higgs coupling in the $\mathrm{HHH}$ final state. Channels with large jet multiplicity, like $t \bar{t} \mathrm{H}$, are less affected, since there jet finding ambiguities dominate the mass resolution. On the other hand, the $\mathrm{ZH} \rightarrow 4$ jets final state is the one which gives the best Higgs mass resolution and is sensitive to the calorimeter performance, even though kinematic constraints can be applied. 
$\mathrm{e}^{+} \mathrm{e}^{-}$Linear Colliders Detector Requirements and Limitations

\subsection{Detector Requirements}

The calorimeter design follows the particle flow approach. Here, the role of the hadron calorimeter (HCAL) is to separate neutral from charged hadrons and to measure their energy precisely. For jet energies below about $100 \mathrm{GeV}$, the performance is dominated by the single hadron energy resolution, whereas at higher jet energies, the so-called "confusion" (mis-assignment of energy depositions to charged and neutral particles) represents the main contribution to the uncertainty. For the assignment, the algorithms make use of both energy and spatial information. Therefore the HCAL should have good energy and good topological precision in three dimensions.

Studies using detailed and realistic detector simulations, and state-of-the-art particle flow reconstruction algorithms have shown that a transverse segmentation of about $3 \mathrm{~cm}$ is optimal for the scintillator technology in the energy range of the ILC [1] (Figure 6.1). As an absorber, steel is chosen for structural reasons and energy resolution, with a longitudinal segmentation of about $2 \mathrm{~cm}$ steel plus about $3 \mathrm{~mm}$ scintillator. For CLIC [2] energies, where leakage becomes more important, tungsten is preferred, with $1 \mathrm{~cm}$ absorber plate thickness.
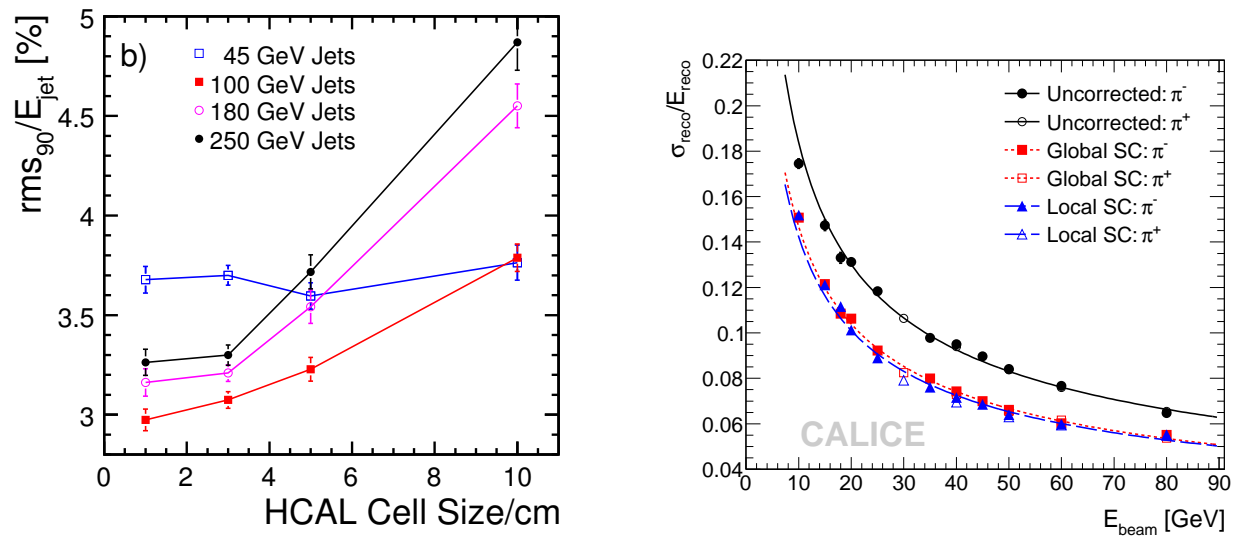

Figure 6.1: Left: Optimization of the hadron calorimeter cell sizes: particle flow jet energy resolution as a function of the AHCAL cell size. Right: Reconstructed energy resolution of the scintillator HCAL prototype for pion showers starting in the first five calorimeter layers. Shown are results obtained with a simple energy sum and with a local and a global software compensating (SC) technique, respectively. 


\subsection{Candidate technologies}

Thanks to the advent of multi-pixel Geiger mode solid state photo-sensors, so-called SiPMs, scintillator has become an attractive and cost-effective option for fine-grained particle flow calorimeters. Linear collider targeted calorimeter R\&D has established SiPMs as a reliable and robust technology, now being used in a variety of experiments, such as CMS, T2K or Belle 2. They conquered a broad range of applications, e.g. in medical imaging, that in turn spurs rapid developments in industry. While devices tested so far fulfill the basic requirements, several vendors are now offering sensors with improved properties, such as larger dynamic range and reduced temperature sensitivity, which further enhance the potential.

For the scintillator, single tiles, mega-tile and strip geometries are being proposed. For mass production, the injection moulding technique for single tiles is most advanced and most explored, but extrusion techniques are being followed, too. More recent sensors are sensitive to blue light and can be coupled directly, without wavelength-shifting fiber, to the scintillator.

\subsection{Current and future R\&D efforts}

Analysis and publication of results from a first generation prototype of a scintillator HCAL with 8000 SiPMs is nearly complete. The simulations have been verified using electromagnetic showers, and a very good hadronic energy resolution was measured, with a stochastic term of $45 \% / \sqrt{E}$ (using simple software compensation methods) and a small constant term of $1.8 \%$ (Figure 6.1 ). Hadronic shower simulation models reproduce the data within a few per-cent. Shower substructure has been resolved, as expected, and the particle flow performance, expressed in terms of the two particle separation power, has been validated with test beam data.

The present phase of $R \& D$ addresses the challenges of a realistic and scalable design, which are posed by the enormous channel density of a calorimeter with 8 million SiPMs. Highly integrated system-on-chip mixed circuit ASICs must be integrated into the detector volume (Figure 6.2) and operate within tiny power budgets. First layers have been tested successfully, but many integration challenges remain, for example the data concentration, power distribution and cooling. For a credible proposal - and cost estimate - scalability has to be demonstrated on all levels, from component production via assembly, quality control, characterization to commissioning and calibration, and the performance has to be re-established with a scalable prototype. 
Thanks to the versatility of the electronics, these developments on the integration and industrialization frontier can proceed in parallel, while one remains open for the more generic $R \& D$ progress on the sensor technology and corresponding optical coupling schemes.

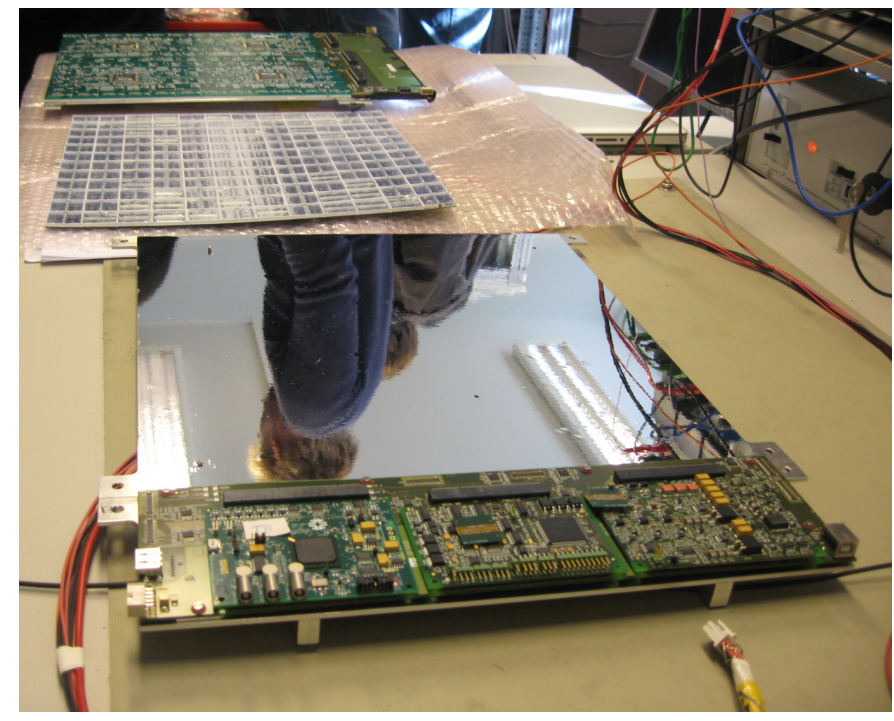

Figure 6.2: Disasembled scintillator HCAL layer with, from top, the PCB with readout ASICs and LED system, tiles with SiPMs, cassette bottom with reflector, and interfaces for data concentration, calibration control and power distribution.

\subsection{Summary}

The scintillator HCAL option, combined with the dynamically evolving SiPM technology, offers a combination of very good classical calorimetric performance with the topological resolution required by the particle flow approach. Test beam experience has met all expectations so far, and improvements are anticipated thanks to better sensors already available now from industry. While these need to be adopted for an optimal design, many challenges remain on the way to a realistic detector proposal and require a sustained $R \& D$ and engineering effort.

\section{Bibliography}

[1] T. Behnke, J. E. Brau, P. N. Burrows, J. Fuster, M. Peskin, M. Stanitzki, Y. Sugimoto and S. Yamada et al., "The International Linear Collider 
Technical Design Report - Volume 4: Detectors,"

[2] L. Linssen, A. Miyamoto, M. Stanitzki and H. Weerts, "Physics and Detectors at CLIC: CLIC Conceptual Design Report," 
$\mathrm{e}^{+} \mathrm{e}^{-}$Linear Colliders Detector Requirements and Limitations 


\section{Chapter 7}

\section{RPC-based digital hadron calorimeter}

Edited by:

Burak Bilki, Kurt Francis, José Repond, Lei Xia

Argonne National Laboratory, USA

\subsection{Physics Requirements}

To fully exploit the physics potential of a future Lepton Collider, new detectors with enhanced capabilities are required. In particular, the detectors are needed which provide the best possible hadronic jet energy resolution. It is generally believed, that a detector optimized for the application of Particle Flow Algorithms can meet this challenge.

In this context, we propose to develop an imaging hadron calorimeter based on Resistive Plate Chambers (RPCs) as active medium. RPCs offer a cost-effective way to providing the necessary fine segmentation of the readout.

\subsection{Detector Requirements}

Imaging calorimetry is a new endeavor, where the foremost challenge is posed by the large number of readout channels. A hadron calorimeter at a future Lepton Collider is expected to count of the order of $5 \cdot 10^{6}$ readout channels. 
$\mathrm{e}^{+} \mathrm{e}^{-}$Linear Colliders Detector Requirements and Limitations

\subsection{Candidate technologies}

RPCs offer numerous advantages as active media of an imaging calorimeter: they are reliable, simple in design and operation, thin, and affordable. Over the past several years the Argonne group has gained invaluable experience in designing, constructing and testing RPCs. Over 200 chambers were built to equip a large prototype imaging hadron calorimeter, the Digital Hadron Calorimeter or DHCAL. The DHCAL layers were inserted into structures with either Iron or Tungsten absorber plates (see Figure 7.1).

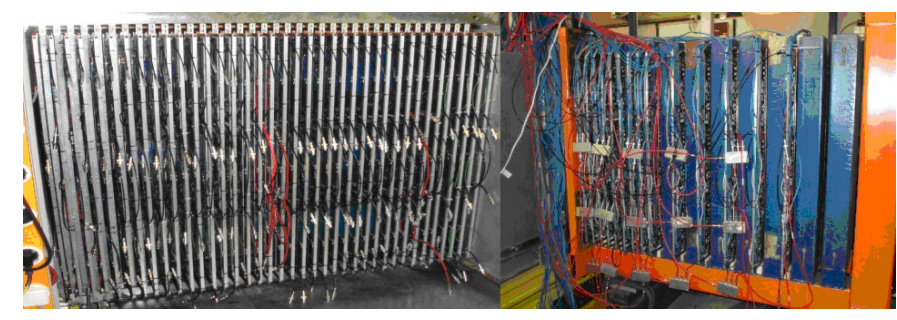

Figure 7.1: Left: the DHCAL main stack (before cabling), right: the Tail Catcher

Testbeams at Fermilab and CERN were used to measure the response to muons, electrons and pions (see Figure 7.2). Based on their result, the DHCAL technology has been validated as active media of an imaging hadron calorimeter.
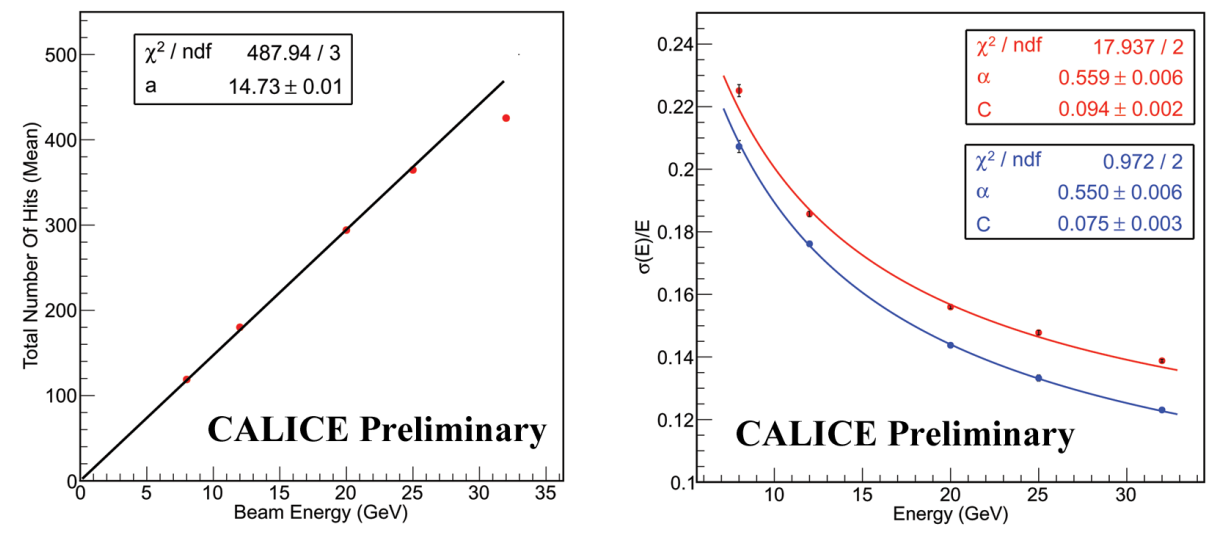

Figure 7.2: DHCAL to pions; Right: hadronic energy resolution of DHCAL for all identified pions (red) and the longitudinally contained pions (blue) 


\subsection{Current and future R\&D efforts}

To prepare for the design of an RPC-based calorimeter for a future Lepton Collider, several technological and engineering issues need to be addressed. The DHCAL readout system, as it was used in the testbeams, was not optimized for operation in a collider environment. A next generation front-end readout needs to be developed which includes token ring passing, power pulsing and a higher channel count, while significantly reducing power consumption. Low and High Voltage distribution systems need to be developed, which can supply power to each layer in a module individually, while monitoring the voltages and currents (such work has initiated at University of lowa). Last, but not least, the gas system also requires further work. A distribution system ensuring uniform gas flow to all layers of a module needs to be developed, as well as a gas recycling system. The latter is mandatory to reduce the cost of operation, but also to protect the environment from the harmful greenhouse gases contained in the standard RPC gas mixture.

The use of 1-glass RPCs for hadron calorimetry, in contrast to the traditional 2-glass version, offers several distinct advantages: thinner active medium, average pad multiplicity close to unity, higher rate capability, easier calibration procedure and more. To date, several large prototype 1-glass RPCs have been built and have been successfully tested. Obtaining an air tight gas volume was one of the initial challenges, but has been successfully overcome. However, before adopting this design for a large-scale hadron calorimeter, their long-term stability still needs to be established.

Finally, a detailed engineering design of a hadron calorimeter based on the RPC technology needs to be worked out. Issues such as cable routing, assembly procedures, mechanical stability need to be addressed, while keeping the area of inactive zones within a calorimeter module to a minimum.

\subsection{Summary}

With the Japanese ILC being a realistic possibility, the development of the DHCAL technology has a chance of leading to the construction of a major part of the detector for such a new facility. Further R\&D on both the chambers, as well as the readout system, is needed to be in a position to develop an engineering design of an RPC-based imaging hadron calorimeter. 
$\mathrm{e}^{+} \mathrm{e}^{-}$Linear Colliders Detector Requirements and Limitations 


\title{
Chapter 8
}

\section{Digital Hadron Calorimetry using GEM}

Edited by:

\author{
A. P. White, M. Sosebee, J. Yu, S. Park, D. Pray, S.A. Khaled, \\ B. Givens, Y. Ng \\ University of Texas, Arlington, USA
}

\subsection{Physics Requirements}

The High Energy Physics group at the University of Texas, Arlington (UTA) has been developing a new type of calorimetry for the future International Linear Collider. This is a critical and essential development for future experiments that will rely on the Particle Flow Algorithm approach to achieve the required jet energy and jet-jet mass resolution. Using small calorimeter cells, typically a few $\mathrm{cm}^{2}$, it is possible to track charged particles in the calorimeter and associate energy deposits with the corresponding tracks whose momenta are well measured in the tracking system. After removal of this "charged energy", the remaining energy is measured using the digital information from the clusters without associated incoming charged track(s). Using digital hadron calorimetry, along with this energy flow algorithm, an overall hadronic (jet) energy resolution of $\sigma_{E} /$ E $3-4 \%$ should be achievable. An example of the need for this precision is the reconstruction of $\mathrm{W}$ and $\mathrm{Z}$ bosons from their hadronic decays. 
$\mathrm{e}^{+} \mathrm{e}^{-}$Linear Colliders Detector Requirements and Limitations

\subsection{Detector Requirements}

In order to be able to track charge particles into and through the calorimeter, the cells must be small $\mathrm{O}\left(1 \mathrm{~cm}^{2}\right)$ - this size of cell has been shown to be effective in Particle Flow Algorithms, with no large improvement in further reduction in size. Since the whole calorimeter system will be contained inside the central solenoid magnet, there is only limited radial space for the 40 layers foreseen for the hadron calorimeter. This imposes a maximum thickness requirement on the active layers of $\mathrm{O}(8 \mathrm{~mm})$. In order to associate hits in the calorimeter with individual bunch crossings, the response of the active layers should be fast $\mathrm{O}$ (few $10 \mathrm{~ns}$ ). Finally, if the same technology is to be used in the forward regions of the hadron calorimeter, the cells of the active layers should have a short recovery time following a hit.

\subsection{Candidate technologies}

Figure 8.1 shows a digital calorimeter concept using GEM technology. Double GEM structures are used to amplify the electron signal deposited by charged tracks. The electrons are collected on anode pads and converted to digital hits on the detector.

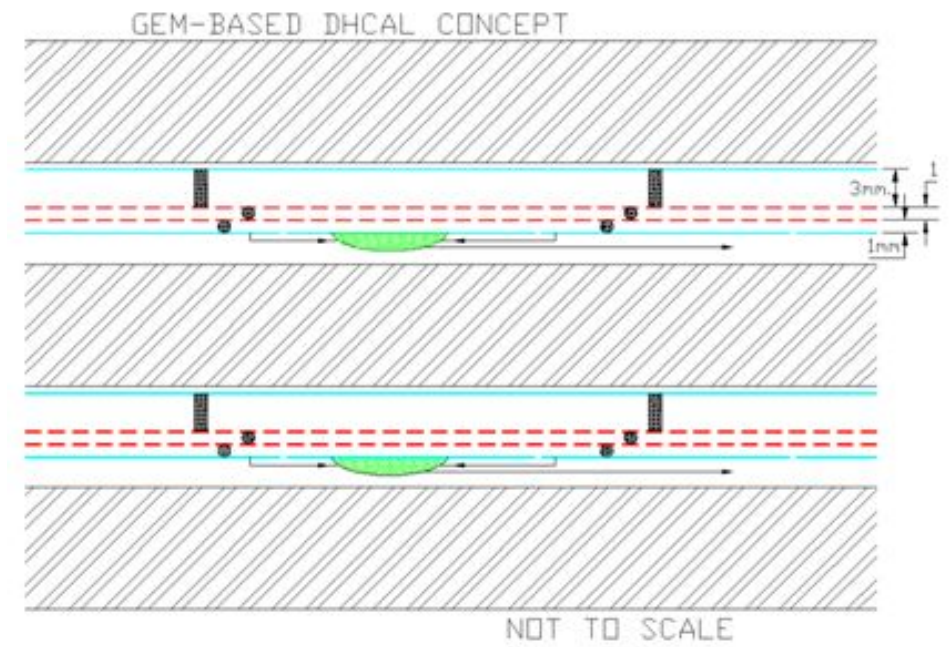

Figure 8.1: Schematic view of GEM-based digital hadron calorimetry. 


\subsection{Current and future R\&D efforts}

A number of prototype GEM detectors, with $1 \mathrm{~cm}^{2}$ readout pads, have been developed and tested. An example is given in Figure 8.2, which shows a number of chambers under test in the Fermilab test beam.

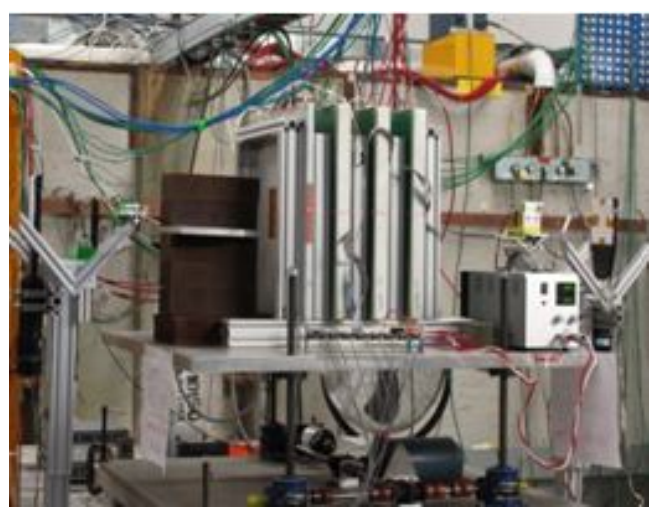

Figure 8.2: GEM chambers under test at the Fermilab test beam.

Figure 8.3 shows typical pion shower profiles in three GEM chambers from pions striking the iron blocks upstream of the chambers. Detailed results from test beam, cosmic rays and radioactive sources have also been produced.

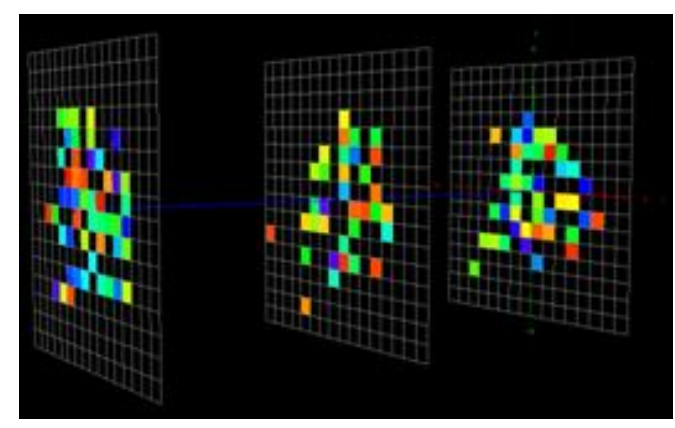

Figure 8.3: Pion shower profile from GEM chambers in Fermilab test beam.

The next step in developing the GEM approach to digital hadron calorimetry is the construction of a number of $1 \mathrm{~m}^{2}$ layers for exposure as part of a $1 \mathrm{~m}^{3}$ test beam stack (together with planes from a resistive plate chamber technology) to study hadronic showers with this new technology. $1 \mathrm{~m} \times 33 \mathrm{~cm}$ foils developed with CERN will be used to assemble double-GEM prototypes of the same size. 
$\mathrm{e}^{+} \mathrm{e}^{-}$Linear Colliders Detector Requirements and Limitations

Following this, a number of $1 \mathrm{~m} \times 1 \mathrm{~m}$ layers will be assembled. Figure 8.4 shows a schematic view of one of these layers.

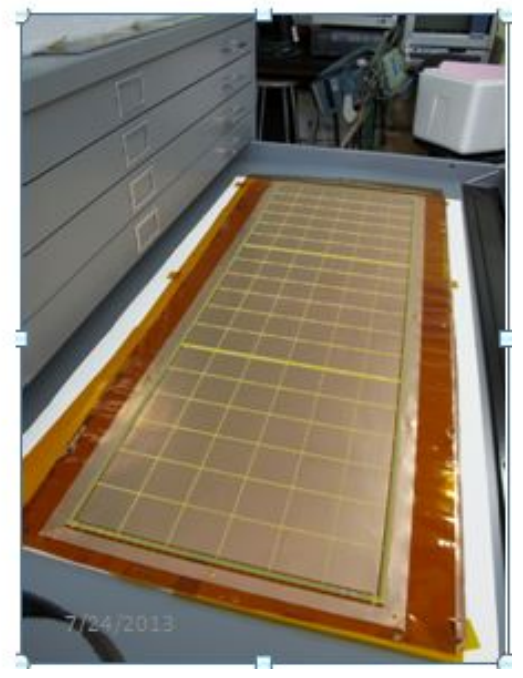

Figure 8.4: A $1 \mathrm{~m} \times 33 \mathrm{~cm}$ GEM chamber under construction at UTA.

The results from the $1 \mathrm{~m}^{3}$ stack will provide essential input for a technology choice for the calorimetry of a future linear collider. 


\section{Chapter 9}

\section{Forward calorimetry}

Edited by:

W. Lohmann

DESY, Germany

Special calorimeters will be used to instrument the very forward regions of the detector [1], denoted hereafter as LumiCal and BeamCal. LumiCal will measure the luminosity using Bhabha scattering with a precision of better than $10^{-3}$ at $500 \mathrm{GeV}$ center-of-mass energy ${ }^{1}$, and BeamCal, just adjacent to the beam-pipe, will perform a bunch-by-bunch estimate of the luminosity and assist beam tuning when included in a fast feedback system [2]. Both calorimeters extend the detector coverage to low polar angles, important e.g. for new particle searches with missing energy signature [3].

Due to the high occupancy originating from beamstrahlung and two-photon processes, both calorimeters need a fast readout. In addition, the lower polar angle range of BeamCal is exposed to a large flux of low energy electrons, resulting in depositions up to one MGy per year. Hence, radiation hard sensors are needed.

Monte Carlo simulations have been performed to optimize the design. In both calorimeters a robust electron and photon shower measurement is essential, making a small Molière radius preferable. Compact cylindrical sandwich calorimeters using tungsten absorber disks interspersed with finely segmented silicon (LumiCal) or GaAs (BeamCal) sensor planes, as sketched in Figure 9.1, are found to match the requirements [1]. Due to the high radiation load CVD ${ }^{2}$ diamond is also considered for the innermost part of BeamCal.

\footnotetext{
${ }^{1} \mathrm{At} 1 \mathrm{TeV}$ center-of-mass energy this requirement is relaxed to $3 \times 10^{-3}$ due to the expected lower statistics of the relevant physics processes.

${ }^{2}$ Chemical Vapour Deposition
} 
$\mathrm{e}^{+} \mathrm{e}^{-}$Linear Colliders Detector Requirements and Limitations

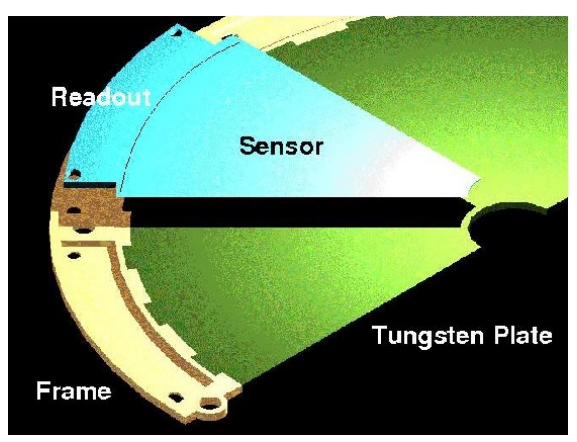

Figure 9.1: A half layer of an absorber disk with a sensor sector and front-end electronics.

The tungsten absorber disks are embedded in a mechanical frame stabilized by steel rods. Finite element calculations were done for the support structure to ensure the necessary precision and stability. The sensors are fixed on the tungsten disks and connected via a flexible PCB to the front-end readout. The gap between the absorber disks is minimized to achieve the smallest possible Molière radius.

Prototypes of LumiCal sensors have been designed and manufactured by Hamamatsu Photonics. Their shape is a ring segment of $30^{\circ}$. The thickness of the $\mathrm{n}$-type silicon bulk is $0.320 \mathrm{~mm}$. The pitch of the concentric $\mathrm{p}^{+}$pads is 1.8 $\mathrm{mm}$ and the gap between two pads is $0.1 \mathrm{~mm}$. The bias voltage for full depletion ranges between 39 and $45 \mathrm{~V}$, and the leakage currents per pad are below $5 \mathrm{nA}$.

Large area GaAs sensors were produced by means of the Liquid Encapsulated Czochralski method, doped by a shallow donor ( $\mathrm{Sn}$ or $\mathrm{Te}$ ), and then compensated with Chromium. This results in a semi-insulating $\mathrm{GaAs}$ material with a resistivity of about $10^{7} \Omega \mathrm{m}$. Sensors were exposed to a $10 \mathrm{MeV}$ electron beam at the S-DALINAC accelerator [4]. The leakage current of a pad of a GaAs sensor before irradiation is about $200 \mathrm{nA}$. After exposure to a dose of $1.2 \mathrm{MGy}$, leakage currents of up to a factor 2 larger were measured [5]. The charge collection efficiency, CCE, is measured before and after irradiation. It drops by a factor of 5 , but a signal from a minimum ionising particle is still visible.

The leakage current in diamond sensors, less than $1 \mathrm{pA}$ at room temperature, depends only slightly on the absorbed dose up to $7 \mathrm{MGy}$. The charge collection efficiency is still sufficiently large after a dose of 7 MGy [6].

ASICs for the readout comprise a large amplication mode for calibration and alignment using muons and a low amplification mode for shower readout. Signals from the subsequent bunch crossings, separated in time by about 300 ns, 
must be resolved and readout. To reduce power dissipation switching off the power between bunch trains is implemented. Two concepts were followd. The first comprising a charge sensitive amplifier and a shaper in a front end ASIC and a separate 10 bit flash ADC. The peaking time of the shaper output signal is $60 \mathrm{~ns}$. ASIC prototypes, containing 8 front-end channels, were designed and fabricated in $0.35 \mu \mathrm{m}$ CMOS technology. A micrograph of the prototype, glued and bonded on the PCB, is shown Figure 9.2 (left). Results of the measurements of the performance were published elsewhere [7]. The multichannel ADC [8], comprises eight 10-bit power and frequency scalable pipeline ADCs and the
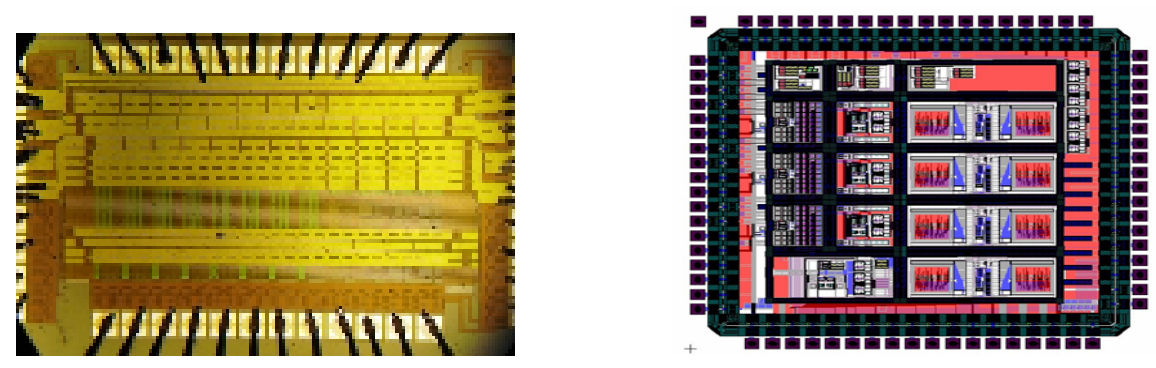

Figure 9.2: Left: Micrograph of front-end ASIC. Right: The micrograph of the ASIC fabricated in $180 \mathrm{~nm}$ CMOS technology.

necessary auxiliary components.

The second concept uses a $180 \mathrm{~nm}$ CMOS process [9]. Prototypes, shown in Figure 9.2 (right), with three channels and an adder that combines the outputs of groups of channels to provide a fast feedback signal ${ }^{3}$ were produced. After amplification, both the signal and the adder output are digitised using a custom 10-bit successive approximation register ADC. The full conversion takes less than 250 ns. The adder digital output is available in less than $1 \mu \mathrm{s}$, compatible with the fast feedback specifications.

Prototypes of sensor planes assembled with FE and ADC ASICs, as shown in Figure 9.3, were built using LumiCal and BeamCal sensors. The detector plane prototypes were installed in an electron beam. The front-end electronics outputs were sampled synchronously with the beam clock, a mode later used at the ILC. Data were taken for different pads and also for regions covering pad boundaries. Signal-to-noise ratios of better than 20 are measured for beam particles both for LumiCal and BeamCal sensors, as illustrated in Figure 9.4. The impact point on the sensor is reconstructed using a beam telescope. Using a colour code for the

\footnotetext{
${ }^{3}$ This signal will be used for beam tuning and diagnostics.
} 
$\mathrm{e}^{+} \mathrm{e}^{-}$Linear Colliders Detector Requirements and Limitations

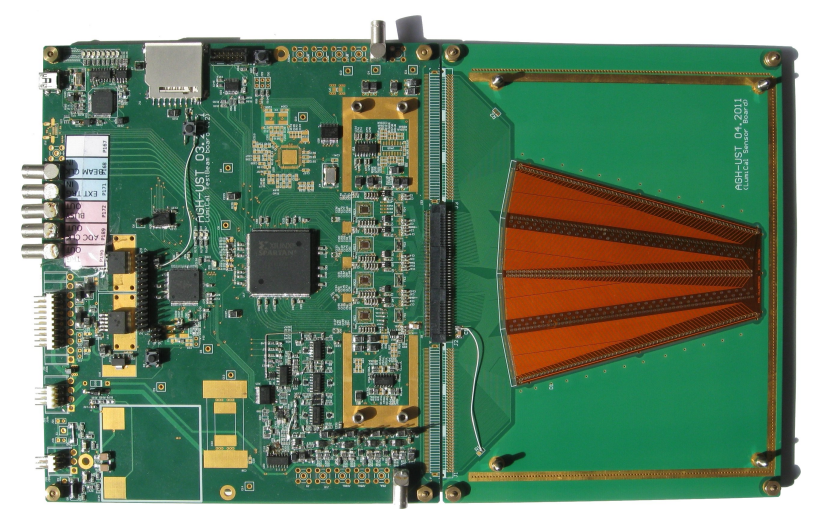

Figure 9.3: Photograph of a readout module with a sensor connected.

signals on the pads the structure of the sensor becomes nicely visible, as also seen in Figure 9.4. The sensor response was found to be uniform over the pad area and to drop by about $10 \%$ in the area between pads.

The next step will be to built a full calorimeter prototype to verify the predictions from simulations with respect to energy and polar angle resolutions. Effort will be invested in connectivity technologies to reduce the thickness of sensor planes. A new generation of ASICs using $130 \mathrm{~nm}$ CMOS technology is under development to further reduce power dissipation and space for the on board electronics. Also a digital memory array on chip will be forseen to readout signals within two bunch trains.

Using a new irradiation facility at SLAC radiation hard silicon sensors, being an option also for BeamCal, will be investigated.

\section{Bibliography}

[1] H. Abramowicz et al., Forward Instrumentation for ILC Detectors, JINST 5 (2010) P12002.

[2] Ch. Grah and A. Sapronov, Beam parameter determination using beamstrahlung photons and incoherent pairs, JINST 3 (2008) P10004.

[3] P. Bambade, V. Drugakov and W. Lohmann, The impact of Beamcal performance at different ILC beam parameters and crossing angles on stau searches, Pramana J. Phys. 69 (2007) 1123.

[4] S-DALINAC: Superconducting DArmstadt LInear ACcelerator, http://www.ikp.tu-darmstadt.de/beschleuniger_1/S-DALINAC.de.jsp. 

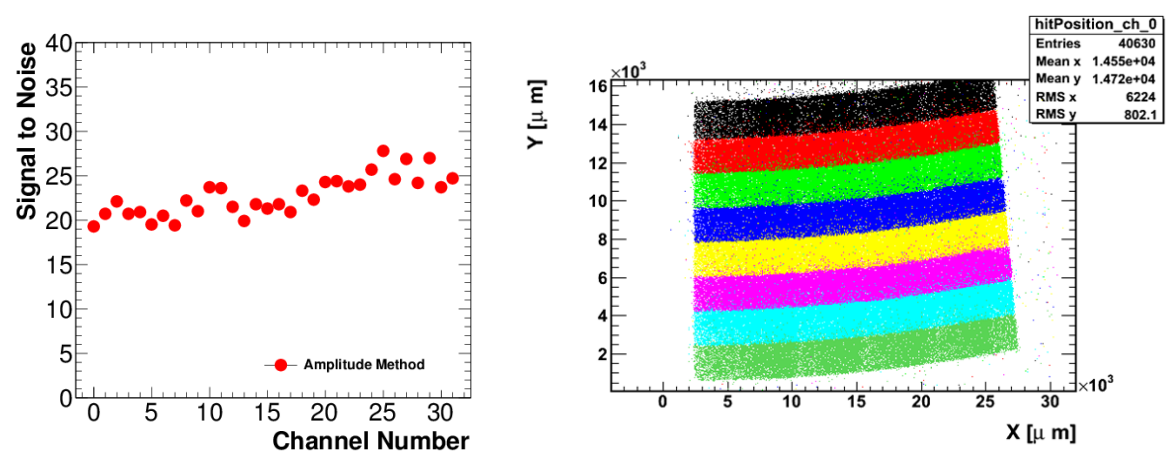

Figure 9.4: Left: The signal-to-noise ratio of all readout channels before calibration. Right: Distribution of the predicted impact points on pads with a color coded signal.

[5] K. Afanaciev et al., Investigation of the Radiation Hardness of GaAs Sensors in an Electron Beam JINST 7 (2012) P11022.

[6] Ch. Grah et al., Polycrystalline CVD Diamonds for the Beam Calorimeter of the ILC, IEEE Trans.Nucl.Sci. 56 (2009) 462.

[7] M. Idzik, Sz. Kulis, D. Przyborowski, Development of front-end electronics for the luminoisty detector at ILC, Nucl. Instr. and Meth. A, vol. 608, pp.169-174, 2009.

[8] M. Idzik et al., A 10-bit multichannel digitizer ASIC for detectors in particle physics experiments, IEEE Trans. Nucl. Sci., vol. 59 pp.294-302, 2012.

[9] A. Abusleme et al., BeamCal Instrumentation IC: Design, Implementation and Test Results, IEEE Trans.Nucl.Sci. 59 (2012) 589. 
$\mathrm{e}^{+} \mathrm{e}^{-}$Linear Colliders Detector Requirements and Limitations 


\section{Chapter 10}

\section{Instrumentation for MDI}

Edited by:

P.N. Burrows, Oxford University

T.W. Markiewicz, SLAC National Accelerator Laboratory

M. Oriunno, SLAC National Accelerator Laboratory

\subsection{Physics Requirements}

The machine detector interface (MDI) challenges are:

- Aligning and stabilizing the final quadrupoles

- Providing for bunch-to-bunch feedback

- Rapid exchange of detectors during push-pull

- Minimal backgrounds in the detector

\subsection{Detector Requirements}

The requirements below are described in more detail in [1]:

A rigid sliding platform and motion system is required that can compensate for long term height adjustment of \pm several $\mathrm{cm}$. The detector axis, as defined by the beam pipe, must be brought to within $\pm 1 \mathrm{~mm}$ and $100 \mu \mathrm{rad}$ of a line determined by the non-traveling QF1 quadrupoles of the final doublet.

Each detector-mounted final quadrupole (QD0) must be adjustable as follows:

- 5 degrees of freedom (horizontal $x$, vertical $y$, roll $\alpha$, pitch $\phi$, yaw $\psi$ ) 
$\mathrm{e}^{+} \mathrm{e}^{-}$Linear Colliders Detector Requirements and Limitations

- Range per $x, y$ degree of freedom: $\pm 2 \mathrm{~mm}$

- Range per $\alpha, \phi, \psi$ degrees of freedom: $\pm 30 \mathrm{mrad}$ (roll), $\pm 1 \mathrm{mrad}$ (pitch and yaw)

- Step size per degree of freedom of motion: $0.05 \mu \mathrm{m}$

Before low intensity beams are allowed to pass through QD0 for high precision beam-based alignment, the mechanical mover system will be required to bring QD0 into alignment with an

- Accuracy per $x, y$ degree of freedom: $\pm 50 \mu \mathrm{m}$

- Accuracy per $\alpha, \phi, \psi$ degree of freedom: $\pm 20 \mathrm{mrad}$ (roll), $\pm 20 \mu \mathrm{rad}$ (pitch and yaw)

The QD0 mechanical alignment accuracy and stability after beam-based alignment and the QD0 vibration stability requirement are set by the capture range and response characteristics of the inter-bunch feedback system.

- QD0 alignment accuracy: $\pm 200 \mathrm{~nm}$ and $0.1 \mu \mathrm{rad}$ from a line determined by the two QF1s, stable over the 200 ms time interval between bunch trains

- QD0 vibration stability: $\Delta\left(\mathrm{QD0}\left(\mathrm{e}^{+}\right)-\mathrm{QDO}\left(\mathrm{e}^{-}\right)\right)<50 \mathrm{~nm}$ within $1 \mathrm{~ms}$ long bunch train

Backgrounds originating at the IP are controlled by detector shielding, beam apertures, and overall layout. The effect of beam gas interactions on detector backgrounds sets the required vacuum level at $1 \mathrm{nTorr}$ in the $\sim 200 \mathrm{~m}$ immediately upstream of the IP and $10 \mathrm{nTorr}$ for the remainder of the Beam Delivery System further away.

\subsection{Candidate technologies}

A $\sim 3 m$-thick steel reinforced concrete platform will compensate for the height difference of the two detectors and provide a stable platform for the QD0s. It will sit on hardened steel rollers which ride on hardened steel rails, pulled by hydraulic jacks. Shim plates or vertical jacks will provide vertical adjustment. Transverse positioning will be verified by a FSI alignment system.

Aligning the two QD0s will require that each be placed in a cantilevered support tube whose orientation is controlled by low-profile (e.g. wedge shaped) movers driven via cam shafts by motors that can function in the solenoid fringe field environment. 
To ensure that beam can safely pass through the final doublet and vertex detector a FSI-based alignment system will tie the tunnel mounted QF1 magnets to the detector mounted QD0 magnets. This may require a line of sight across the interaction point. Extensions of the FSI alignment system will ensure that the tracking detector position after each push pull operation is known well enough that it not require beam-based alignment.

A compact, $\sim 40 \mathrm{~cm}$ diameter, cryostat will house each incoming-beamline quadrupole/sextuplole/octupole coil assembly, the extraction-beamline quadrupole and an anti-solenoid winding to eliminate the solenoid-quadrupole fringe field repulsive forces that would otherwise require a robust and rigid mounting of the cryostat.

Beam-train to beam-train, as well as coherent bunch-to-bunch, misalignments due to ground motion or residual environmental vibration of the QD0 magnet or its support will be removed by an inter-bunch beam-based feedback system. Because of the high sensitivity of the beam-beam deflection to beam offsets and the $>300$ ns bunch-to-bunch spacing, only a small number of bunches at the head of each train are needed to align the subsequent bunches to the nm level.

Achieving $1 \mathrm{nTorr}$ vacuum upbeam of QF1 will be a challenge. With a $20 \mathrm{~mm}$ diameter stainless beampipe and $50 \mathrm{l} / \mathrm{s}$ ion pumps every $2 \mathrm{~m}$, the average pressure is $23 \mathrm{nTorr}$. Either distributed pumping (antechamber, pumpscreens or NEG coatings) or/and larger diameter beampipes with bakeout facilities, will probably be required to meet the $1 \mathrm{nTorr}$ requirement for minimal beam-gas backgrounds in the detector.

\subsection{Current and future R\&D efforts}

An Oxford University developed FSI system has been in use at ATLAS for some time. Small, low mass reflector blocks consistent with the precision SiD detector need to be developed. A mounting scheme that provides the appropriate geometric constraints needs to be developed.

The Oxford FONT ILC prototype system has been designed and tested at the ATF2 and other accelerators [2]. A kicker magnet and BPM engineered for the ILC tight geometry need to be designed, built and tested.

BNL has wound the quadrupole part of the QD0 prototype. This coil will be cryostated and tested for field quality. Measurements of internal coil motion with $1.9 \mathrm{~K}$ superfluid $\mathrm{He}$ will be carried out.

Measurements of quadrupole motion at comparable detectors (e.g. CMS) will be carried out during the LHC shutdowns and the data and support systems modelled appropriately to give confidence to the mounting schemes proposed by 
$\mathrm{e}^{+} \mathrm{e}^{-}$Linear Colliders Detector Requirements and Limitations

SiD for the ILC.

\section{Bibliography}

[1] B. Parker et al, Functional requirements on the design of the detectors and the interaction region of an $\mathrm{e}^{+} \mathrm{e}^{-}$linear collider with a push-pull arrangement of detectors, Proceedings of Particle Accelerator Conference (PAC09), Vancouver, May 2009.

[2] R. Apsimon et al, Latest beam test results from ATF2 with the FONT ILC prototype intra-train beam feedback systems, Proceedings of the International Particle Accelerator Conference, Kyoto, May 2010. 


\title{
Chapter 11
}

\section{Electronics}

\author{
Edited by: \\ M. Breidenbach \\ SLAC, USA
}

\subsection{Introduction}

$\mathrm{e}^{+} \mathrm{e}^{-}$detectors optimized for the physics accessible from the ILC have focused on superb jet energy resolution achieved through particle flow calorimetry and on high precision tracking. This note will focus on the strategy and R\&D efforts required for $\mathrm{SiD}$. The calorimetry requires highly pixellized sensors to dissect the jet into individual particles, and thin sensors to optimize the Moliere radius and keep electromagnetic showers localized. The tracker requires the fine resolution available from $\mathrm{Si}$ strips and minimal multiple scattering to achieve the momentum resolution.

$\mathrm{e}^{+} \mathrm{e}^{-}$detectors optimized for the physics accessible from the ILC have focused on superb jet energy resolution achieved through particle flow calorimetry and on high precision tracking. The calorimetry requires highly pixellized sensors to dissect the jet into individual particles, and thin sensors to optimize the Moliere radius and keep electromagnetic showers localized. The tracker requires the fine spatial resolution available from Si strips and minimal multiple scattering to achieve the momentum resolution. Sensors for the vertex detectors that can cover large areas with pixels of $20 \times 20 \mu \mathrm{m}$ or less, that are thin and structurally stable, and that have appropriate timing and amplitude measurement are needed.

The hadron calorimeter also requires good segmentation, probably with $1 \mathrm{~cm}^{2}$ pixels, and should be thin for cost optimization. "Digital" and "semidigital" gas counter that have a broad amplitude response to a crossing MIP 
$\mathrm{e}^{+} \mathrm{e}^{-}$Linear Colliders Detector Requirements and Limitations

are under consideration, as are scintillating plastic tiles with analog readout. The muon system requires large areas of modest performance tracking. This can be accomplished by scintillation strips read out by SiPM's, or large area RPC's. The hadron calorimeter also requires good segmentation, probably with $1 \mathrm{~cm}^{2}$ pixels, and should be thin for cost optimization. "Digital" and "semidigital" gas counter that have a broad amplitude response to a crossing MIP are under consideration, as are scintillating plastic tiles with analog readout. The muon system requires large areas of modest performance tracking. This can be accomplished by scintillation strips read out by SiPM's, or large area RPC's.

\subsection{Current and future R\&D efforts}

The virtues of the $\mathrm{e}^{+} \mathrm{e}^{-}$colliders include total cross sections that are small compared to hadron colliders, so that event rates and radiation loads are small and not a difficult challenge for ASIC designers. In addition, the time structure at ILC and CLIC is such that duty factors are quite small, $1 \%$, so that power modulation to the ASIC's can keep heat loads manageable by gas flow in the tracker and conduction in the calorimeter. The low average power and the desire to keep service channels small to maintain hermeticity makes DC-DC power conversion with a high conversion ratio attractive. These devices, which must operate in high magnetic fields and be small, are under development. Finally, the trigger strategy appropriate for ILC appears to be "no trigger". The Data Acquisition System will poll the read-out chips at $5 \mathrm{~Hz}$ and build events, with event filtering being an offline process. It is believed that a reasonable solution for DAQ could be Reconfigurable Cluster Elements (RCE's) packaged in the Advanced Telecommunications Computing Architecture (ATCA).

The R\&D strategy adopted by SiD focuses on silicon sensors, either $10 \times$ $10 \mathrm{~cm}$ arrays of $A C$ coupled strips at a $50 \mu \mathrm{m}$ pitch for the tracker; or hexagonal sensors approximately $12 \mathrm{~cm}$ across their flats with 1024 pixels for calorimetry. A readout chip would bump bond to the sensor without any intermediate hybrid, and traces on the sensor would connect power and signals to a thin cable going to data concentrators and power conditioners. SiD has been developing $\mathrm{KPiX}, \mathrm{a}$ 1024 channel "System on a Chip" for this purpose. KPiX has:

- low noise, dual range charge amplifiers with a 17 bit dynamic range,

- power modulation so that the power is $<20 \mu \mathrm{W} /$ channel in ILC mode,

- up to four measurements during each ILC train with each measurement recording the bunch number and the amplitude, 

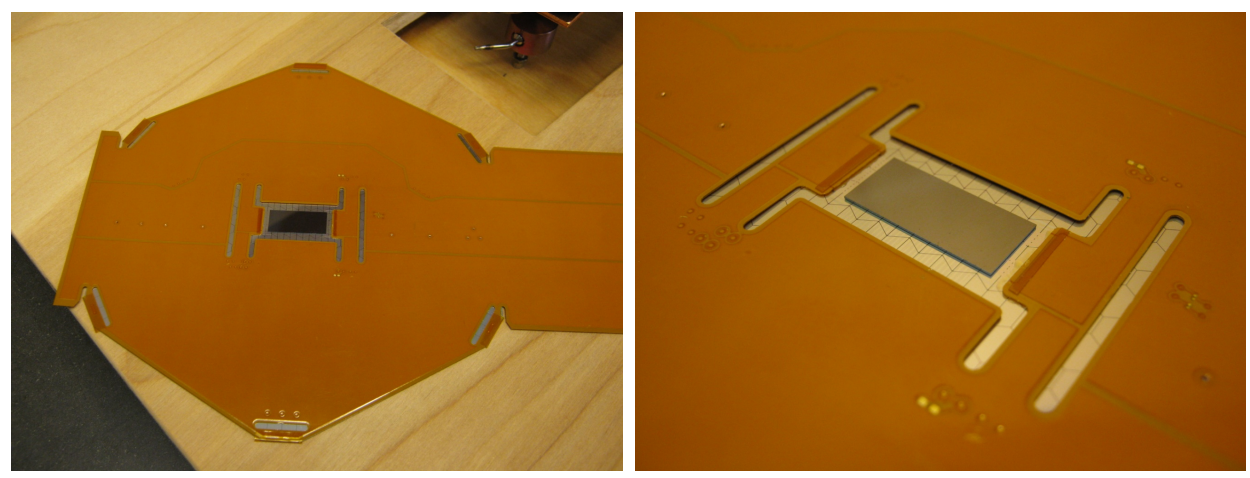

Figure 11.1: The ECAL silicon sensor bonded to a KPiX and cable (left) and a close-up of the $\mathrm{KPiX}$ region (right). The hexagonal pixels of the sensor are visible.

- digitization and readout during the inter-train period,

- an internal calibration system,

- a noise floor of approximately $0.15 \mathrm{fC}\left(1000 \mathrm{e}^{-}\right)$,

- a peak signal (auto ranging) of $10 \mathrm{pC}$,

- and a trigger threshold selectable from 0.1 to $10 \mathrm{fC}$.

SiD has had significant trouble with bump bonding KPiX to prototype detectors. The current versions of KPiX come from the foundry with eutectic $\mathrm{Sn}-\mathrm{Pb}$ solder bumps ready for reflow, but the sensors have Al pads. Preparing suitable under bump metalization (UBM) has been unexpectedly difficult, but about 10 calorimeter sensors have bonded $\mathrm{KPiX}$ chips and are being evaluated and another 20 are in process. These sensors are being attached to cables (see Figure 11.1), and are being assembled on tungsten plates into a test-beam calorimeter. However, it is clear that the current UBM process is unacceptable, and work with vendors to produce more bondable sensors is a high priority. Bonding of the tracker sensors has not yet been attempted.

SiD has two R\&D efforts for vertex detector sensors:

- Chronopix is a monolithic sensor that records the time (14 bits) when a signal exceeds threshold. The current prototype has $25 \times 25 \mu \mathrm{m}$ pixels, using $90 \mathrm{~nm}$ technology. Will go to $65 \mathrm{~nm}$, with $18 \mu \mathrm{m}$ pixels. 
$\mathrm{e}^{+} \mathrm{e}^{-}$Linear Colliders Detector Requirements and Limitations

- Vertical Integrated Pixel (VIP) is a sensor using "3D" technology by FNAL and MIT-Lincoln Laboratory. The current prototype is $48 \times 48$ array, 28 $\times 28$. It will provide amplitude and time information.

These efforts are making progress, but substantial effort will be needed before relatively large sensors are available. Other approaches are being pursued by ILD.

The hadronic calorimeter may be readout using $\mathrm{KPiX}$. A challenge here is the adaption of the $200 \times 500 \mu \mathrm{mKPiX}$ pad pitch to a pitch suitable for a PC board, without introducing excessive height.

\subsection{Summary}

Many of the important electronics developments are nearly ready for beam testing. Critical measurements of crosstalk and noise on real sensors (with their second metal layer crossing lower structure) in a realistic environment remain to be done. Nevertheless, the more significant problems have been in bonding, and there is some hope that UBM application by the sensor foundry will solve this problem. 


\section{Chapter 12}

\section{Detector Alignment}

Edited by:

K. Riles, University of Michigan

\subsection{Physics Requirements}

Although precise alignment has been important to $\mathrm{e}^{+} \mathrm{e}^{-}$detectors for decades, the demands on detector alignment at future $\mathrm{e}^{+} \mathrm{e}^{-}$colliders will become still more stringent. The most aggressive alignment needs are for the central tracker and vertex detector, driven by two distinct type of physics requirements on charged particles - momentum resolution and impact parameter resolution. The most important physics measurement driving momentum resolution is determination of the Higgs boson mass [1], based on recoil against dimuons in the channel $\mathrm{e}^{+} \mathrm{e}^{-} \rightarrow H\left(Z \rightarrow \mu^{+} \mu^{-}\right)$. The most important physics consideration driving impact parameter resolution is heavy flavor tagging via displaced vertices and reliable particle assignment to the vertices. This tagging affects measurement of Higgs boson branching ratios (and, in turn, discrimination of a Standard Model Higgs from a Supersymmetry Higgs) and also affects measurement of heavy flavor forward-backward asymmetries that are highly sensitive to new physics [1].

\subsection{Detector Requirements}

The unprecedented momentum resolution goal for $\mathrm{e}^{+} \mathrm{e}^{-}$linear collider $4 \pi$ detectors $\left[\delta\left(1 / p_{\perp}\right) \sim 2 \times 10^{-5} \mathrm{GeV}^{-1}\right]$ requires precise sagittas and hence small systematic uncertainties in the relative alignments of central and vertex tracking ele- 
$\mathrm{e}^{+} \mathrm{e}^{-}$Linear Colliders Detector Requirements and Limitations

ments. Similarly, aggressive goals in impact parameter resolution $[(5 \oplus 10 / p) \mu \mathrm{m}$, $p$ in $\mathrm{GeV}$ ] place strong demands on not only the intrinsic pixel resolution of the vertex detector layers, but also on the systematic uncertainties of their relative alignments.

An important consideration in designing an alignment system is minimization of material burden due to the alignment elements themselves. Such material in the fiducial volume of the tracking system can lead to multiple scattering that degrades the average momentum and impact parameter resolution.

Traditionally, precise alignment of $\mathrm{e}^{+} \mathrm{e}^{-}$trackers has relied heavily on the accumulation of charged particles in the tracker over the course of a year's run, to probe not only the many degrees of freedom describing individual tracker elements, but also to probe for global detector alignment distortions, e.g., displacements, rotations, twists, sag, etc.. One complication for the nominal ILC baseline with push-pull operation is that realignment will be necessary more frequently and will suffer from fewer charged particles during each epoch, placing a higher premium on a priori alignment, independent of accumulated collision data.

Another alignment need particular to a future linear collider detector is that required for the final-focus quadrupole magnets that bring the electron and positron beams into collision. Beams with height of $\mathrm{O}(10 \mathrm{~nm})$ must be collided without the benefit of the somewhat automatic path-sharing seen for counter-rotating beams in traditional storage rings. Once the beams are near enough to affect each other's motion, beam-based diagnostics can be used to servo them toward maximum overlap, but a priori alignment is needed at the level of $50 \mu \mathrm{m}$ in transverse positions and $20 \mu \mathrm{rad}$ in pitch and yaw angles.

Finally, pulsed-power operation of tracker electronics may lead to mechanical vibrations and temperature fluctuations and resulting alignment challenges.

\subsection{Candidate technologies}

A wide variety of detector alignment techniques have been developed over the years, many of which could be applied to aligning various elements of a linear collider detector. Here we discuss only those that have been proposed specifically for the especially demanding task of aligning central and vertex tracking trackers for a linear collider detector:

1. Frequency-scanned interferometry

2. Infrared laser "tracks"(for aligning silicon micro-strips)

3. Embedded fiber optic sensors 
Frequency Scanned Interferometry (FSI) is an alignment method pioneered and implemented for the ATLAS Experiment $[2,3]$. In FSI a geodetic grid of points attached to the object to be aligned and to nearby bedrock is used to measure positions, rotations and distortions of detector and accelerator elements. The points in the grid are optically linked via a network of laser beams, using optical fibers for beam launching and collection. Small beam splitters and retroreflectors define interferometers for which a scanning of laser frequency over a known range defines absolute distance measurements to sub-micron precision. The resulting precisions on other lengths from fits to the grid measurements depend on the number and layout of the FSI lines of sight.

Infrared laser beams mimic the straight trajectories of tracks with infinitely high momenta. Hence these "tracks" provide a direct calibration of sagitta measurements as they traverse successive silicon layers, where targeted etching of metallic layers permits the infrared beam to pass through the silicon with only partial absorption providing "hits". This alignment method has been used for the AMS [4] and CMS [5] detectors. The IR beams can give rapid feedback on relative tracker element displacements, although they do not distinguish between global movements of a monitored structure from global movements of the laser beams.

Fiber optics can be embedded in tracking structures, such as carbon-fiber support tubes, to measure minute strains signaling misalignment [6]. These fibers have etched Bragg gratings that reflect particular wavelengths and transmit other wavelengths. Induced strains in the embedding structure (from temperature, pressure, vibration, etc.) change the reflected wavelength and intensity. Individual fibers can be etched with different gratings along their lengths, allowed targeted monitoring of spatially varying strains. Such fiber monitoring can be done continuously, permitting monitoring of both short-term and long-term global tracker distortions, including during push-pull operations.

\subsection{Current and future R\&D efforts}

All three technologies described above appear promising, but need further R\&D. For the FSI method, further work is needed $[7,8]$ to reduce the material burden of beam launch/return and retroreflector components and to improve the rapidity of measurement. For the partially transparent infrared laser system, work is needed [9] to improve beam transmittance and to mitigate resolution degradation due to interference and diffraction of laser beams. Using beams to align inclined surfaces is another challenge, given refraction displacements. For the fiber-Bragg sensors, work is needed to verify with large prototype modules the mapping of 
$\mathrm{e}^{+} \mathrm{e}^{-}$Linear Colliders Detector Requirements and Limitations

fiber strain measurements to global deformations.

\subsection{Summary}

Although the goals for track momentum and impact parameter resolution for a future linear collider detector placed stringent demands on systematic uncertainties on detector alignment, there exist several technologies with the promise to meet those demands.

\section{Bibliography}

[1] International Linear Collider Reference Design Report, August 2007.

[2] "Frequency Scanned Interferometry (FSI): The Basis of a Survey System for the ATLAS ID using fast automated remote interferometry", A.F. FoxMurphy et al., N uc. Inst. and Meth. A383, 229 (1996).

[3] "A Novel Method for ATLAS FSI Alignment based on Rapid, Direct Phase Monitoring", S.M. Gibson et al., ATLAS Report INDET-PROC-2010-037, http://cdsweb.cern.ch/record/ 1305878/files/ATL-INDET-PROC-2010-037.pdf (November 2010); ATLAS ID using fast automated remote interferometry", A.F. Fox-Murphy et al., N uc. Inst. and Meth. A383, 229 (1996).

[4] W. Wallraff, TAS status, AMS Tracker Meeting, Montpellier, 22-23 June 2004.

[5] B. Wittmer et al., The Laser Alignment System for the CMS Silicon Microstrip Tracker, Nucl. Instr. Meth. A581 (2007) 351.

[6] K.O. Hill et al.,, Photosensitivity in optical fiber waveguides: application to reflection fiber fabrication. Appl. Phys. Lett. 32 (1978) 6.

[7] "High-precision Absolute Distance Measurement using Frequency Scanned Interferometry' ', H. Yang, J. Deibel, S. Nyberg and K. Riles, Appl. Opt., 44, 3937 (2005).

[8] "High-precision Absolute Distance Measurement using Dual-Laser Frequency Scanned Interferometry Under Realistic Conditions", H. Yang and K. Riles, Nuc. Inst. and Meth. A575, 395 (2007). 
[9] J. Duarte et al., Alignment of Silicon tracking systems R\&D on Semitransparent Microstrip Sensors, Proc. International Linear Collider Workshop, Nov 16-20, 2008, Chicago, arXiv:0901.1231, January 2009. 
$\mathrm{e}^{+} \mathrm{e}^{-}$Linear Colliders Detector Requirements and Limitations 


\section{Summary}

A large body of R\&D exists for the proposed linear collider detectors, SiD and ILD [1]. This effort has developed over more than a decade and solutions exist that can deliver required physics performance at a linear collider.

This $R \& D$ has been driven by many different groups ranging from large collaborations like CALICE to small groups like the FSI R\&D and was predominantly generic and not being aimed at a particular concept or a particular machine, which is illustrated by the fact that many ideas have been incoporated both for ILC and CLIC detectors.

Besides for the Vertex Detector, where no technology meets all of the requirements yet, a proof-of-principle has been made for all other subsystems. The current $R \& D$ now slowly moves towards understanding system issues.

The current state of these efforts has been documented in the Detailed Baseline Designs of the ILC TDR [1] and the CLIC CDR [2] and the references therein.

\section{Bibliography}

[1] T. Behnke, J. E. Brau, P. N. Burrows, J. Fuster, M. Peskin, M. Stanitzki, Y. Sugimoto and S. Yamada et al., "The International Linear Collider Technical Design Report - Volume 4: Detectors,"

[2] L. Linssen, A. Miyamoto, M. Stanitzki and H. Weerts, "Physics and Detectors at CLIC: CLIC Conceptual Design Report," 
1 Intensity Frontier

$\operatorname{cxxx}$ 


\title{
Electromagnetic Calorimetry in Project X Experiments - The Project X Physics Study
}

\author{
Summary and R\&D prospects \\ Fritz DeJongh, Milind Diwan, Corrado Gatto, David Hitlin, David E. Jaffe, Yuri Kamyshkov, \\ Laurie Littenberg, William Molzon, Anna Mazzacane, Andrei Poblaguev, Peter Winter, \\ Elizabeth Worcester, Minfang Yeh, Ren-yuan Zhu
}

\section{Introduction}

The Electromagnetic Calorimetry Working Group investigated a series of muon, kaon and neutron-antineutron oscillation experiments in existing or proposed pre-Project $\mathrm{X}$ versions, and in several instances examined whether or not the calorimetric techniques employed in these experiments could be extrapolated to produce viable experiments at Project $\mathrm{X}$ in its various stages. This was done, as per our charge, purely from the perspective of the calorimeters involved. In so doing, areas of potentially fruitful R\&D in calorimetry were identified. The resulting initiatives have both short term experiment-specific goals, and longer term more generic objectives.

\section{Muon physics}

Experiments examined included an improved measurement of $g-2$ of the muon, a search for $\mu$ to $e^{-}$conversion and related searches for $\mu^{-}$to $e^{+}$conversion and $\mu^{+} \rightarrow 3 e$ decay, and a search for $\mu^{+} \rightarrow e^{+} \gamma$ decay.

\section{$g-2$}

The BNL $g$-2 experiment uses $\mathrm{Pb}$-scintillating fiber electromagnetic calorimeters, read out with PMTs, to detect decay electrons at twenty four stations around the ring. The Fermilab version of the experiment will upgrade these calorimeters to cope with rates as much as three times higher. $\mathrm{PbWO}_{4}$ scintillating crystals, $\mathrm{PbF}_{2}$ Cerenkov counters and $\mathrm{W} / \mathrm{scintillating}$ fiber devices have been considered. Arrays of the latter two have been tested in the Fermilab test beam from 2 to $8 \mathrm{GeV}$. The $\mathrm{PbF}_{2}$ crystals are the leading candidate. Readout would be with either Hamamatsu R9800 PMTs or S10362-33-050C sixteen channel SiPMs.

\section{Mu2e}

The Mu2e configuration contains an electromagnetic calorimeter designed to furnish confirmation of a $105 \mathrm{MeV}$ conversion electron signal from the tracker, as well as to provide a possible trigger. The calorimeter consists of 2000 LYSO crystals, read out by large area APDs. The default geometry is four vanes arrayed around the beamline; an 
alternative disk geometry is also under consideration (see Figure 1). The LYSO has a decay time of $40 \mathrm{~ns}$, requiring a gate interval of about $200 \mathrm{~ns}$. This is sufficient to reject pileup in the current incarnation of the experiment, and perhaps in the early stages of a Project X-capable experiment. It would not suffice for the increases in intensity contemplated at later stages. For this, since energy resolution is of prime importance, a new faster scintillator would have to be employed (see below).
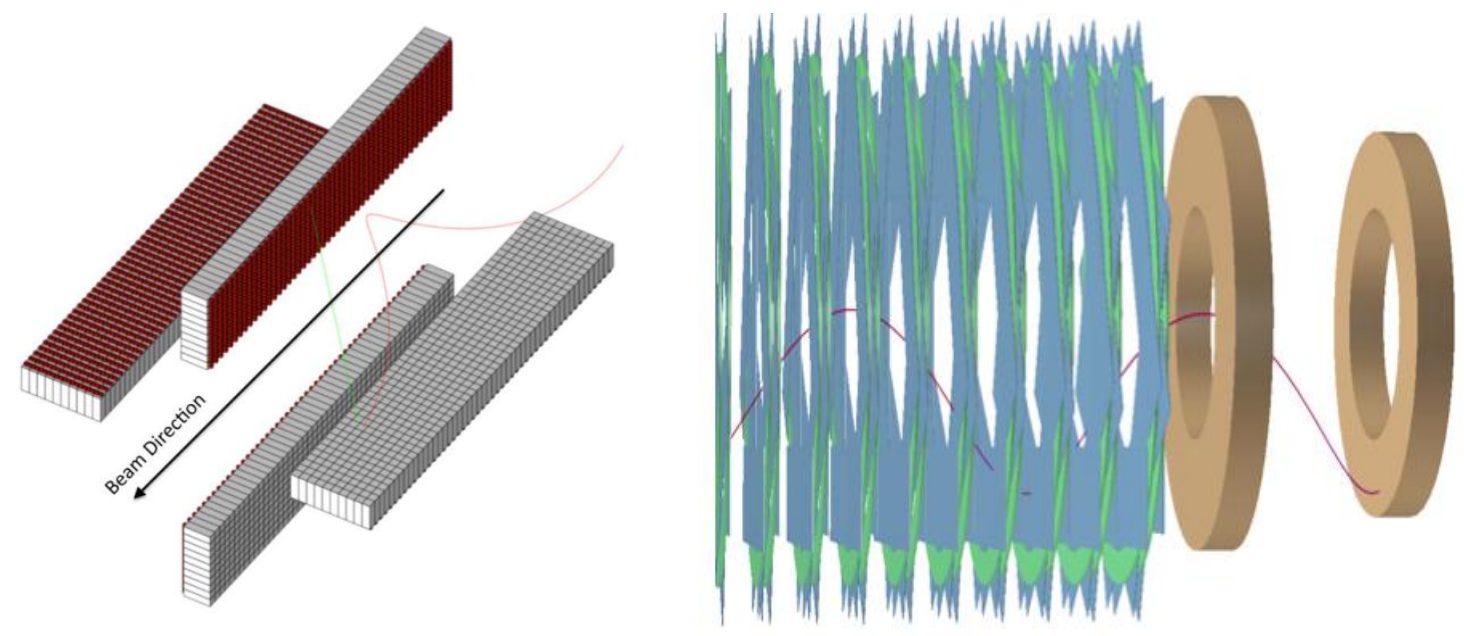

Figure 1. Vane (left) and disk (right) calorimeter configurations in the Mu2e experiment.

$\mu^{+} \rightarrow e^{+} \gamma$

The current MEG experiment at PSI was presented, with an eye to examining the planned upgrade and the possibility of employing a calorimetric technique for a $\mu^{+} \rightarrow e^{+} \gamma$ search at Project X. The current data sets a limit of $2.4 \times 10^{-12}$ at $90 \% \mathrm{CL}$. The liquid xenon calorimeter did not quite reach design specification; upgrades are planned to improve the energy resolution. These involve improving the spatial resolution of the detectors mounted on the entrance face by replacing the existing PMTs with large area $(12 \times 12$ $\mathrm{mm}^{2}$ ) MPPCs, as shown in Figure 2, reducing the reflectivity of the inner walls and improving the time resolution. The expected sensitivity of the experiment would then improve to a few $\times 10^{-13}$.

It is unlikely, however, that the MEG technique can be successfully employed at Project $\mathrm{X}$ rates, since the accidental rate increases as the square of the stopping muon rate. This has motivated a study of an alternative approach, in which the photon is detected by reconstructing the tracks of an $e^{+} e^{-}$pair produced in a thin converter. This amounts to a tradeoff of detection efficiency for energy resolution and concomitant background rejection. Early simulations indicate that this non-calorimetric technique could reach a single event sensitivity of $2 \times 10^{-16}$. The concept uses double pixel layers to measure the 
position and direction at several points on the helical trajectory of a track in a solenoidal magnetic field, as shown in Figure 3.
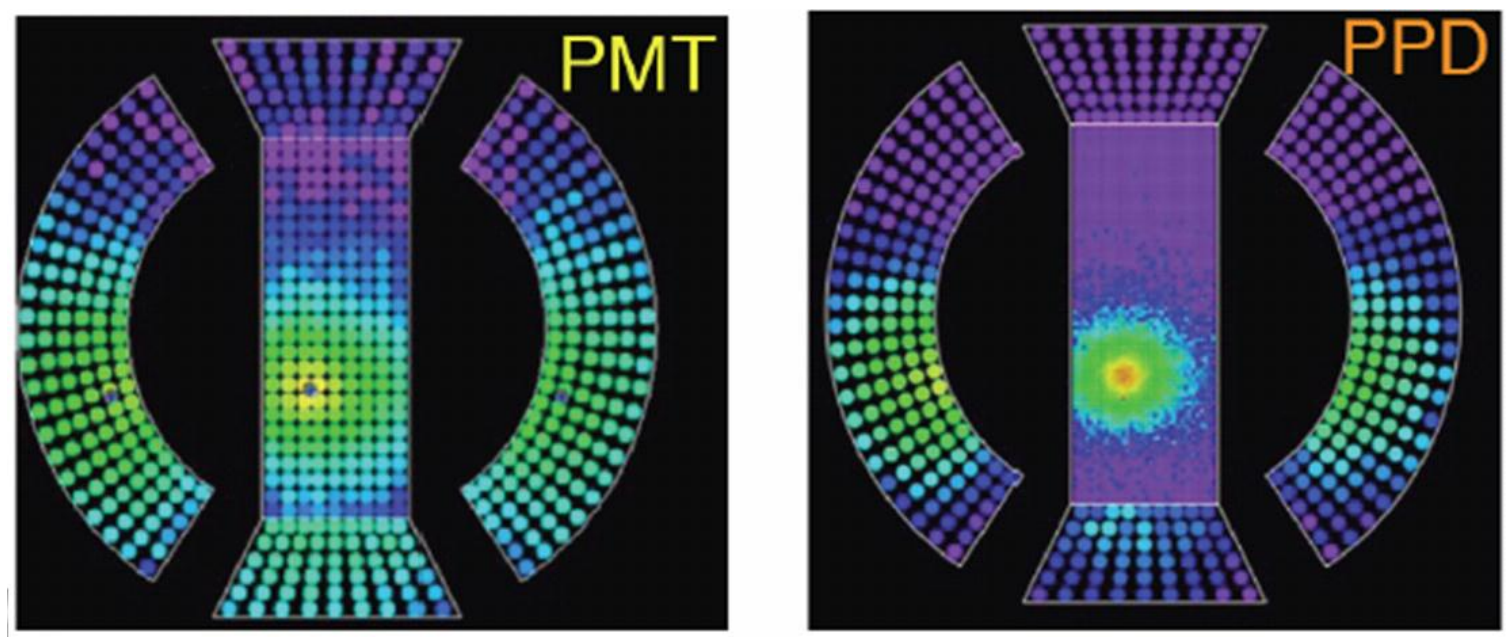

Figure 2. Improvement in shower localization to be achieved by MEG by replacing PMTs on the LXe calorimeter entrance face with MPPC devices and reducing wall reflectivity.

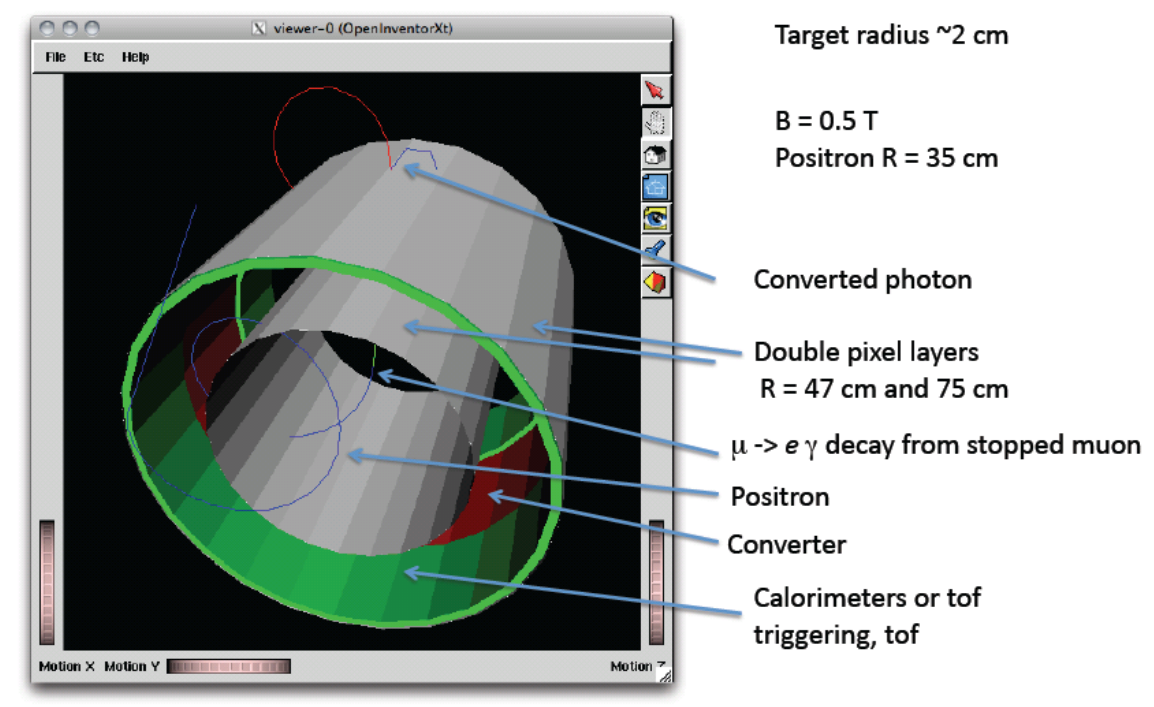

Figure 3. Concept of a $\mu^{+} \rightarrow e^{+} \gamma$ experiment using a photon conversion technique.

The high muon and kaon rates expected at Project $\mathrm{X}$ make high quality calorimetry quite difficult. The candidate technologies are based on homogeneous scintillating devices, either noble liquids or crystals. While LXe has a fast decay time of $45 \mathrm{~ns}$, the large radiation length and practical requirement that the light be detected at the periphery of a large volume, limit the achievable time resolution. The current generation of crystalbased high quality, high rate capable, calorimeters employs $\mathrm{PbWO}_{4}$ and $\mathrm{LYSO}$, which have decay times of 30 and $40 \mathrm{~ns}$, respectively. Project $\mathrm{X}$ will increase the rates of 
stopping muon experiments by nearly a factory of fifty, which makes these decay times far too slow to prevent such calorimeters from being overwhelmed by accidentals.

This leads to the question of whether there are other radiation-hard crystals having decay times that are meaningfully faster. $\mathrm{BaF}_{2}$, has the fastest known scintillation component, with a decay time of $600 \mathrm{ps}$ at $220 \mathrm{~nm}$, representing $\sim 15 \%$ of the total light output. Unfortunately, $85 \%$ of the light, peaking at $300 \mathrm{~nm}$, has a decay time of $650 \mathrm{~ns}$. This crystal was studied in some detail as a candidate technology for the GEM detector at the $\mathrm{SSC}$, but has received little attention in the past two decades. Realizing the promise of a high resolution sub-nanosecond electromagnetic calorimeter requires further development in two areas. The first is the properties of the crystal itself. There is evidence that doping $\mathrm{BaF}_{2}$ with lanthanum rather than cerium can reduce the slow component while having little effect on the fast component. This parameter space should be further explored. If a more optimum doping is found, other important properties, such as radiation harness, then need to be measured. The second area is the photodetector. There are solar-blind photocathodes for PMTs that can further enhance the fast signal response over the slow, but suitable devices that have the appropriate spectral response, rise time and magnetic field insensitivity do not yet exist. There are, however, several promising avenues that can be explored. One is to extend the current work at Chicago, Argonne, LBNL and Fermilab on fast, large area planar microchannel plate PMTs to use solar-blind photocathodes such as K-CS-Te. The other is to develop SiPM or APD devices with UV spectral sensitivity. There are small devices with enhanced UV sensitivity; these would have to be extrapolated to larger sizes. This two-prong approach should form the basis of an interesting collaborative R\&D initiative.

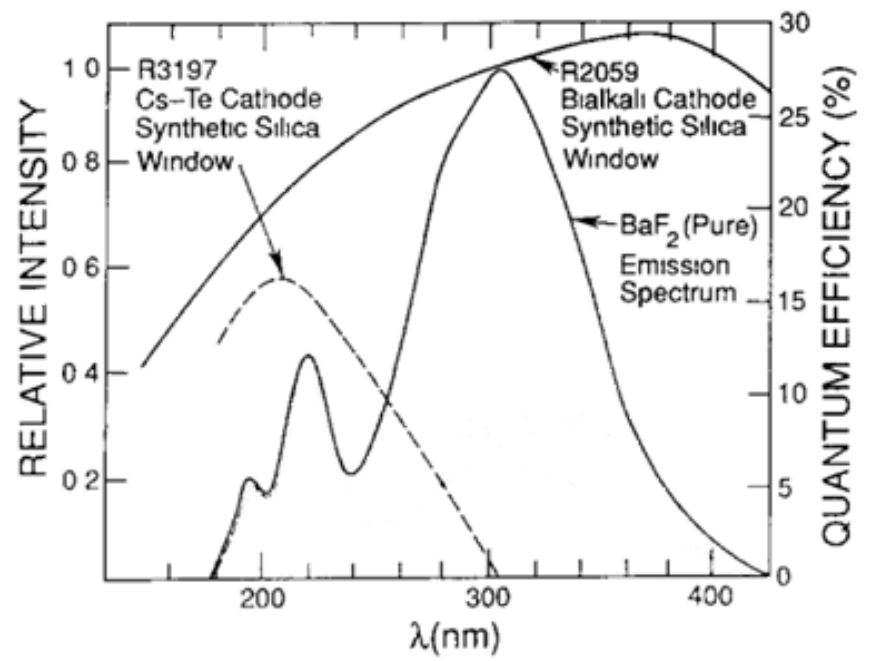

Figure 4. Scintillation light spectrum of $\mathrm{BaF}_{2}$, showing the fast $220 \mathrm{~nm}$ and slow $300 \mathrm{~nm}$ components, along with the spectral response of two types of photocathodes. 


\section{Work needed on muon experiments}

The more than one order-of-magnitude increase in muon stopping rates at Project $\mathrm{X}$ will require new approaches to photon detection. There are two main thrusts:

- Explore the reach of $\mu^{+} \rightarrow e^{+} \gamma$ experiments with the technique of detection of the $e^{+} e^{-}$ pair from photon conversion.

- Extend high quality scintillating crystal calorimetry to the sub nanosecond realm by developing new dopings for crystals such as $\mathrm{BaF}_{2}$, and new fast solar-blind readout devices.

\section{Kaon physics}

Experiments examined included a $K^{+} \rightarrow \pi^{+} v \bar{v}$ decay measurement and a search for the decay $K_{L}^{0} \rightarrow \pi^{0} v \bar{v}$, along with a review of the $\mathrm{KTeV}$ experience.

$K^{+} \rightarrow \pi^{+} v v$

ORKA (see Fig. 5) is a proposed FNAL experiment to measure the branching ratio of $K^{+} \rightarrow \pi^{+} v v$, predicted by the standard model to be $\sim 7 \times 10^{-11}$. The observed final state in $K^{+} \rightarrow \pi^{+} v v$ decay is a single charged pion; the main sources of background are the twobody kaon decays $B\left(K^{+} \rightarrow \pi^{+} \pi^{0}\right)=21 \%$ and $B\left(K^{+} \rightarrow \mu^{+} v\right)=63 \%$. The rejection of $K^{+} \rightarrow \pi^{+} \pi^{0}$ is relevant for the requirements on calorimetry. Assuming a signal-to-background of at least 10 , the $K_{\pi 2}$ decay background must be suppressed by a factor of $\sim 3 \times 10^{10}$ or more. $K_{\pi 2}$ rejection is achieved by accurate reconstruction of the kinematics of the charged pion and detection of the photons from $\pi^{0}$ decay. To reduce background from $K_{\mu 2}$ and other muon background, the final state decay chain $\pi^{+} \rightarrow \mu^{+} \rightarrow e^{+}$is reconstructed in the drift chamber and range stack. The range stack introduces almost one full radiation length of material between the decay point and the calorimeter.

The measurement of $B\left(K^{+} \rightarrow \pi^{+} v v\right)$ in a stopped-kaon experiment requires detection of photons in the energy range (20-225) MeV from $\pi^{0}$ decay. E949 successfully observed $K^{+} \rightarrow \pi^{+} v v$ with a rejection of $\sim 10^{6}$.

High photon veto efficiency requires conversion and detection of photons. Photon detection can be maximized by a fully active, hermetic calorimeter with sufficient thickness in radiation lengths $\left(X_{0}\right)$. Studies for the KOPIO experiment indicate that $23 X_{0}$ would be sufficient for a stopped-kaon experiment. Inoperative calorimeter elements imply a loss of hermiticity, so redundancy or a high degree of single-channel reliability is required. The stopped-kaon experiment requires a solenoidal magnetic field and the calorimeter must be accommodated by the solenoid. This constraint may imply a compromise between the fully active and thickness requirements. Inactive components of 
the calorimeter, such as lead layers, effectively lower the minimum photon energy threshold from $20 \mathrm{MeV}$.

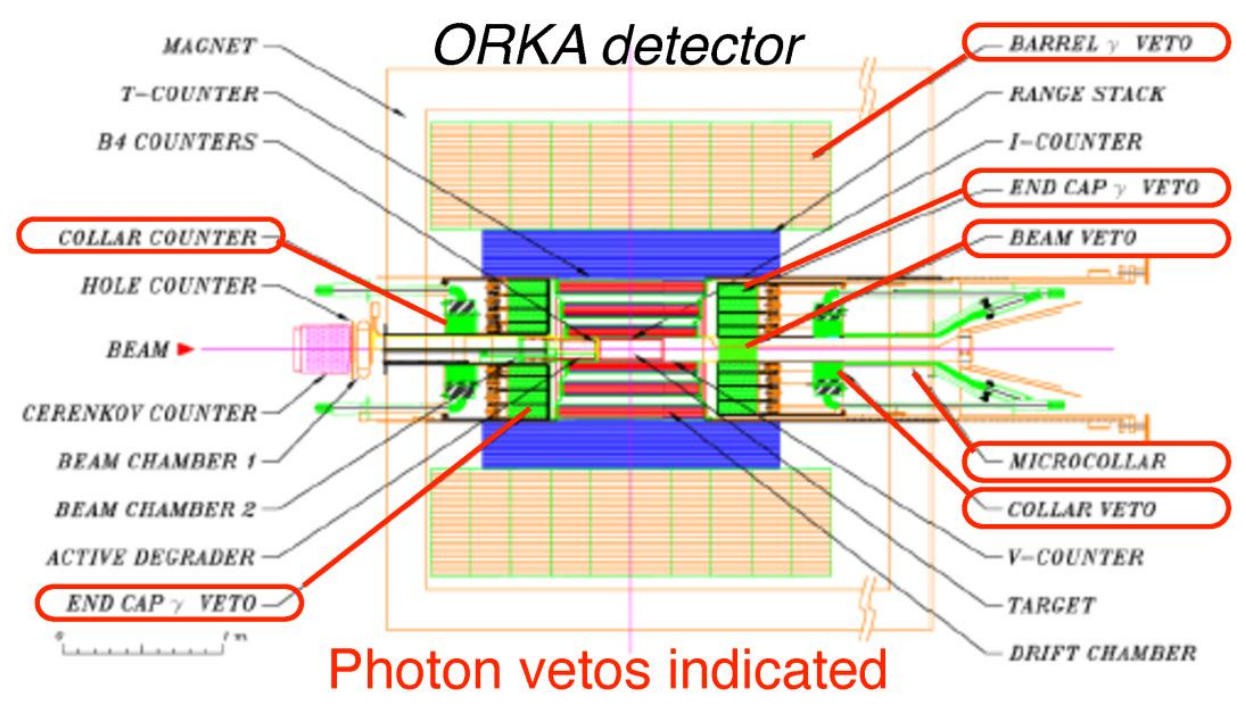

Figure 5. Elevation view of the proposed ORKA detector indicating the locations of photon veto detectors. The beam enters from the left.

High photon veto efficiency must be achieved while minimizing acceptance losses due to vetoing on random or 'accidental' detector activity. These acceptance losses can be minimized by minimizing the time interval required for the coincidence of $\mathrm{K}^{+}$decay and the putative photon.

A scintillator-based calorimeter would need to have a short decay time, high light yield, and high collection efficiency to satisfy the veto requirement. The ability to reconstruct photons and electrons for other (non- $K^{+} \rightarrow \pi^{+} v v$ ) measurements in the experiment would place additional requirements on the calorimeter energy, position, and shower timing resolutions. Improvements to the reconstruction ability of the calorimeter may also reduce systematic uncertainties for $K^{+} \rightarrow \pi^{+} v v$; for example, $\pi^{0}$ reconstruction would allow for studies of $\pi^{+}$detection efficiency using $K^{+} \rightarrow \pi^{+} \pi^{0}$ decays. Studies to quantify the size of these potential improvements would be helpful in choosing among design options.

As proposed, ORKA has two primary calorimetric elements: the endcap and the barrel veto. For the endcap, ORKA could re-use the $\sim 20025 \mathrm{~cm}$ long CsI crystals from BNL E949 that provide $13.5 X_{0}$ total depth. The expected energy and timing resolutions are $\Delta E / E \sim 11 \%$ and $\Delta t \sim 0.7 \mathrm{~ns}$, respectively. Use of shashlyk detectors, with greater depth, is also a possibility that should be explored.

Several designs are under consideration for the barrel veto. A shashlyk style calorimeter similar to the one designed for KOPIO (see Sect. 0.1.2 below) would satisfy the photon 
veto requirement. A possible configuration is 155 interleaved layers of $0.8-\mathrm{mm}$ lead and 1.6-mm scintillator read out by $\sim 400$ wavelength shifting fibers. This provides $23 X_{0}$ total depth. The expected energy and timing resolutions are (3-4) $\% / \sqrt{ } E$ and $(90-100)$ $\mathrm{ps} / \sqrt{ } E$, respectively. The noise term in the energy resolution is negligible above $50 \mathrm{MeV}$ so was not included in the parameterization for KOPIO, but will likely be relevant for ORKA because the photon energies will be lower. A shashlyk calorimeter has already been prototyped for KOPIO. For ORKA, because it must fit inside the CDF solenoid, the detector must be somewhat more compact without losing veto power. More work on design and prototyping to fulfill this size requirement is needed.

Another option for the calorimeter design is a totally-active ADRIANO calorimeter consisting of 150 interleaved layers of $2 \mathrm{~mm}$ lead glass and $2 \mathrm{~mm}$ fast scintillator. Work to simulate the performance of this style detector in ORKA is ongoing, but the energy resolution is expected to be better than a shashlyk style detector, particularly at low energies. It may also allow reconstruction of angles using timing or light division methods. From the perspective of ORKA, the most important benefit of the totally-active ADRIANO calorimeter would be the potential reduction in photon inefficiency relative to a shashlyk style calorimeter.

NA62 at CERN is a decay-in-flight experiment, currently under construction, to measure $B\left(K^{+} \rightarrow \pi^{+} v v\right)$. The technique will be complementary to that of ORKA because decay-inflight and stopped-kaon experiments are sensitive to different parts of phase space.

$K_{L}^{0} \rightarrow \pi^{0} \boldsymbol{v} \bar{v}$

KOPIO (see Fig. 2) was a proposed BNL experiment to measure the branching ratio of $K_{L}^{0} \rightarrow \pi^{0} v \bar{v}$. It is expected that a similar experiment will be proposed at FNAL in the Project $\mathrm{X}$ era. The standard model value of the $K_{L}^{0} \rightarrow \pi^{0} v \bar{\nu}$ branching ratio is $\sim 3 \times 10^{-11}$.

$K_{L}^{0} \rightarrow \pi^{0} \pi^{0}$ is the main background. The two-photon final state of the signal requires accurate reconstruction of single photon energy, position, direction and time. The photon veto requirements are more stringent than $K^{+} \rightarrow \pi^{+} v v$ (Section 0.1.1) because background photon energies can extend below $20 \mathrm{MeV}$ due to decay-in-flight of the $K_{L}^{0}$. Also in contrast to a stopped-kaon experiment, the beam represents an extended signal and background source that alters the hermeticity requirements. The minimum performance requirements are:

- Direction resolution: $25 \mathrm{mrad} @ 250 \mathrm{MeV}$

- Energy resolution: $3 \% / \sqrt{ } E$

- Timing resolution: $\sim 100 \mathrm{ps} / \sqrt{ } E$

- Position resolution: $250 \mu \mathrm{m}$

- Veto efficiency: $10^{-4} / \gamma$ 


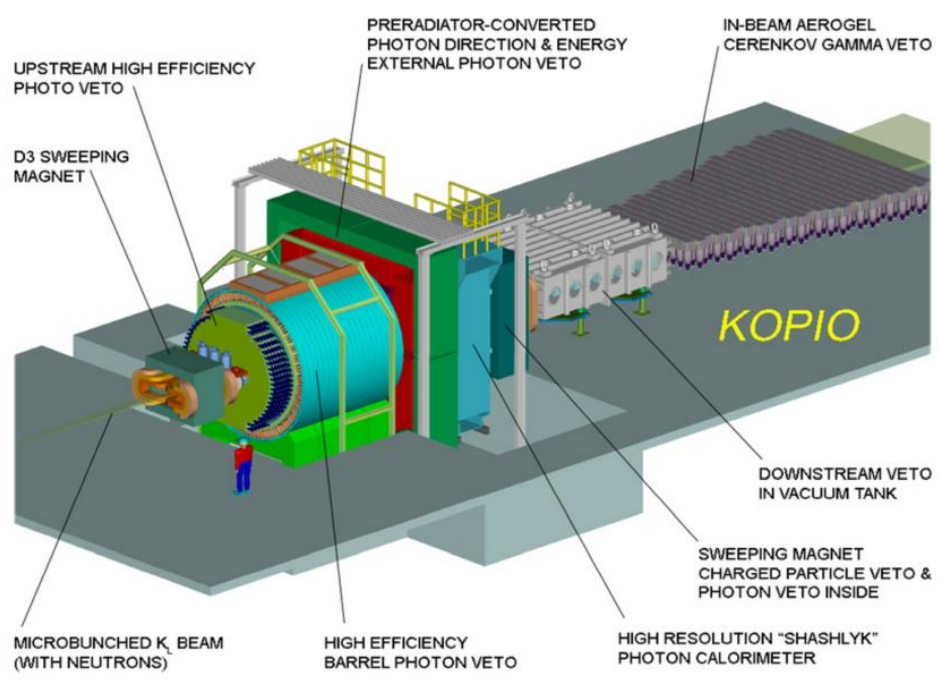

Figure 6. Schematic of the proposed KOPIO detector. The pre-radiator is shown in green and the shashlyk calorimeter is shown in blue.

The measurement of photon energies and directions in KOPIO is made by the combination of a pre-radiator and a shashlyk style calorimeter. The pre-radiator consists of scintillator sheets alternating with cathode strip drift chambers. It contains $2.7 X_{0}$ of material in total; it is important to keep the thickness in the beam direction of the preradiator as small as possible in order to limit transverse shower size so that photons from background decay modes may be resolved. The result of this configuration is that most photon interactions are $e+e^{-}$pair production in which the angular separation of the $e+e^{-}$ pair is small enough that the individual particles need not be resolved by the calorimeter. The initial direction of $e+e^{-}$pair is the same as that of the photon, so the track reconstructed in the pre-radiator drift chambers is a measurement of the photon angle. Following the pre-radiator is a $19 X_{0}$ shashlyk style calorimeter (lead-scintillator sandwiches read out by wavelength shifting fibers passing through holes in the scintillator and lead), with transverse granularity of $\sim 10 \mathrm{~cm}$. By design, the KOPIO preradiator/calorimeter combination satisfies the performance specifications above.

A study comparing the improvement in signal yield relative to background for various improvements in calorimeter performance finds that improvements to angular and energy resolution are most beneficial. Reduction of photon veto inefficiency also provides significant improvement in S/B. Timing resolution is less critical, and position resolution least significant.

Studies from KOPIO show that energy resolution improves as the thickness of the lead plates is reduced, the thickness of the scintillator plates is increased, and as the number of layers increases. These design parameters correspond to increased sampling efficiency 
and increased photostatistics, which are two of the three primary drivers of the energy resolution. The third is light collection uniformity, which can be improved by increasing the light collection efficiency near the edges of a scintillator tile.

Angular resolution might be improved if the pre-radiator could resolve the opening angle between the $\mathrm{e}+\mathrm{e}^{-}$pair; this would require use of a high-speed, high-resolution tracker or TPC that does not add large amounts of material to the detector. The ability to reconstruct angles using the calorimeter would also be helpful. These ideas are speculative and have not yet been studied.

KOPIO achieved a modest increase in signal acceptance by using the barrel photon veto (see Fig. 6). Improvements in angle, position, and energy resolution of calorimetry would boost this acceptance.

KOTO is an experiment at J-PARC to search for $K_{L}^{0} \rightarrow \pi^{0} \pi^{0}$ decay, building on its predecessor, E391 at KEK. It is currently under construction and expects to begin taking data in 2013. Calorimeter and photon veto performance are critical to this experiment; one of the major upgrades between E391 and KOTO is the use of the KTeV CsI crystals for the KOTO calorimeter. Experience from this experiment will be very useful in the design of a KOPIO-style experiment at Project X.

\section{Work needed on kaon experiments}

The calorimeters needed for kaon experiments in the Project X era are reasonably well understood; the shashlyk style calorimeter designed for KOPIO has been prototyped and likely can be modified to meet the design requirements for both ORKA and a KOPIOstyle experiment. However, it may be possible to significantly improve the sensitivity of these experiments and/or broaden their physics reach by making improvements to the calorimetry. Much work remains to optimize the calorimeter design and study which improvements to the calorimeter are most likely to pay off in improvements to the measurements. Finally, R\&D in 3D calorimetry and low energy photon veto detection would be of general use to the HEP community. Specific studies that are needed include:

- Compact shashlyk design for ORKA: the ORKA calorimeter must fit inside the CDF solenoid. The shashlyk calorimeter designed for KOPIO must be made more compact without sacrificing veto efficiency.

- Benefits to ORKA from improved calorimetry: the size of potential improvements to the measurement of $K^{+} \rightarrow \pi^{+} v v$ resulting from improvements to calorimeter resolution should be quantified.

- Benefits of ADRIANO calorimeter: the size of potential improvements to the measurement of $K^{+} \rightarrow \pi^{+} v v$ resulting from improved photon veto efficiency in a totallyactive calorimeter should be quantified. 
- KOPIO calorimeter optimization: improvements to the energy and angular resolution of the KOPIO calorimeter result in significant improvements to the measurement of $\mathrm{K}_{\mathrm{L}}^{0} \rightarrow \pi^{0} v v$. The detector should be optimized to improve the energy resolution as much as possible. Some thought should be given to potential ways to improve the angular resolution.

-3D calorimetry: The ability to "track" photons while maintaining high photon veto efficiency is highly desirable. Development of 3D calorimetry techniques such as time difference methods and light division methods as well as R\&D for calorimeters with $z$ segmentation, with a focus on preservation of veto efficiency, would be useful for $K \rightarrow \pi v v$ experiments.

- Low energy photon veto detectors: This document has primarily focused on scintillation detectors. Generic R\&D for low energy photon veto detection is important. New design ideas that improve photon veto efficiency could significantly improve the sensitivity of $K \rightarrow \pi v v$ experiments. 


\section{Intensity Frontier Instrumentation}

\section{V2.0 July $28^{\text {th }} 2013$}

The fundamental origin of flavor in the Standard Model remains a mystery. Despite the roughly eighty years since Rabi asked "Who ordered that?" upon learning of the discovery of the muon, we have not understood the reason that there are three generations or, more recently, why the quark and neutrino mixing matrices are so different. The solution to the flavor problem would give profound insights into physics beyond the Standard Model (BSM) and tell us about the couplings and the mass scale at which the next level of insight can be found.

The Standard Model fails to explain all observed phenomena: new interactions and yet unseen particles must exist. They may manifest themselves by causing Standard Model reactions to differ from often very precise predictions. The Intensity Frontier explores these fundamental questions by searching for new physics in extremely rare processes or those forbidden in the Standard Model. This often requires massive and/or extremely finely tuned detectors.

\section{Executive Summary}

There is overlap of instrumentation needs between kaon and muon experiments. Most of these require detection of particles in the $10 \mathrm{MeV}$ to $1 \mathrm{GeV}$ range associated with stopped kaon and muon interactions. Neutrino experiments tend to have somewhat different instrumentation needs.

1) Development of large mass, cost effective detectors for neutrino detection and proton decay.

2) R\&D towards cost effective calorimeters with good photon pointing and Time of Flight (TOF) (goal is $<20 \mathrm{mrad}, 10 \mathrm{ps}$ ).

3) R\&D towards cost effective, high efficiency photon detection for kaon experiments $\left(10^{-4}\right)$

4) Very fast, very high resolution photon/electron calorimetry for muon experiments (goal is $100 \mathrm{ps}$, sub-percent energy resolution)

5) Very low mass, high resolution, high-speed tracking for muon and kaon experiments $\left(0.001 \mathrm{X}_{0}\right.$ per space point, 100ps per track)

6) High fidelity simulation of low energy particle interactions; strategies to effectively simulate $>10^{12}$ particle decays \& interactions.

7) High throughput, fault tolerant streaming data acquisition systems (goal of TB/second throughput to $\mathrm{PB} /$ year data storage)

\section{Neutrinos and Proton Decay}

Neutrino energies span an enormous range and in some important neutrino experiments, such a $0 v \beta \beta$, the neutrinos are not even available for detection. However, some neutrino detector themes are common: they rely on large mass, in which scintillation or Cherenkov light detection is a primary or secondary detection mechanism. It is frequently important to have good event 
reconstruction and good particle identification, including good separation of electrons from gammas.

Due to the small neutrino interaction cross sections, the detectors frequently need to be very massive. This feature provides a strong synergy with proton decay searches which also need massive detectors.

While in some cases, the need for event reconstruction has led to segmented detectors, the primary focus has been on large monolithic detectors. The three primary detection media are water, liquid scintillator (LS) and liquid argon (LAr). Prominent examples of water detectors include IMB, Kamiokande and Super-K through proposed experiments such as MEMPHYS and LBNE WCD. Prominent examples of LS detectors include reactor experiments dating back to the 1950's, through KamLAND, the $\theta_{13}$ experiments and the proposed next round of long baseline reactor experiments: JUNO and RENO-50. We can also include LSND, MiniBooNE and LENA in this class. Prominent examples of LAr detectors include ICARUS, MicroBooNE and the proposed LBNE and GLACIER. All three technologies rely on the detection of scintillation or Cherenkov light, whereas LAr detectors also collect ionization electrons to get excellent event reconstruction. A key element of the $R \& D$ program would be increased light yield through the development of improved optical attenuation length and increased numbers of detected photoelectrons per dollar.

Current R\&D focuses on improved attenuation length of LS detectors, which has been historically accomplished by mixing LS with mineral oil, but which is currently focusing on water-based liquid scintillators. These detectors can get the benefit of large amounts of scintillation light in combination with Cherenkov light reconstruction. They are also importantly sensitive to events below Cherenkov threshold, such as the kaon in $p \rightarrow K^{+} v$. Current $R \& D$ to increase the number of detected photoelectrons per dollar has focused on large area photodetectors based on microchannel plates.

Another key area of R\&D is on increasing performance of large LAr detectors. The current program focuses on purification of LAr and on measuring and understanding fundamental parameters, such as optical attenuation length and ionization electron diffusion. Additional R\&D focuses on efficient scintillation photoelectron collection at $128 \mathrm{~nm}$. Additional R\&D focuses on TPC design, with efforts looking into both 1-phase and 2-phase readout. The US effort for massive detectors focuses on the 1-phase readout.

The neutrino working group of the Project-X Physics Study undertook to survey neutrino detector requirements of potential Project-X experiments and the capabilities of available technologies, with the goal of identifying high priority areas for R\&D. The priorities identified by the PXPS neutrino report include:

- New types of liquid scintillator such as LAB and water-based scintillators. 
- Improvements in segmentation and readout to build large, economical, room-temperature scintillator detectors that can provide more fine-grained and complete information about neutrino interactions.

- Further work with liquid argon TPC detectors to more fully exploit both ionization and scintillation and efficiently use the wealth of information provided by these detectors.

- Development of ton-scale single phase low-energy threshold liquid argon neutrino detector for coherent scattering measurement

- Various improvements in materials, readout and analysis

These recommendations will be further elucidated at the Minnesota Snowmass meeting.

\section{Kaons}

Kaon decays have played a pivotal role in shaping the Standard Model (SM). Prominent examples include parity violation, quark mixing, meson-antimeson oscillations, CP violation, suppression of flavor-changing neutral currents (FCNC), and discovery of the GIM mechanism and prediction of charm. Kaons continue to have high impact in constraining the flavor sector of possible extensions of the SM.

A key role is played by the FCNC modes mediated by the quark-level processes $s \rightarrow d v v$, and in particular the golden modes $\mathrm{K}^{+} \rightarrow \pi^{+} v v$ and $\mathrm{K}_{\mathrm{L}} \rightarrow \pi^{0} v v$. Because of the peculiar suppression of the $\mathrm{SM}$ amplitude (loop level proportional to $\mathrm{V}_{\mathrm{us}}{ }^{5}$ ) which in general is not present in SM extensions, kaon FCNC modes offer a unique window on the flavor structure of such extensions. This argument by itself provides a strong and model independent motivation to study these modes in the LHC era. Rare kaon decays can elucidate the flavor structure of SM extensions, information that is in general not accessible from high-energy colliders. The actual discovery potential depends on the precision of the prediction for these decays in the SM, the level of constraints from other observables and how well we can measure their branching ratios.

The US experimental program pioneered the study of $\mathrm{K} \rightarrow \pi \nu \nu$ and the future program is evolving to include a charged $\mathrm{K}^{+} \rightarrow \pi^{+} v v$ experiment, ORKA, making use of the intense Main Injector beam, as a key step towards a neutral $\mathrm{K}_{\mathrm{L}} \rightarrow \pi^{0} v v$ KOPIO-like experiment using the Phase-2 Project-X beam. An extensive kaon program built around the flagship KOPIO experiment is likely to include an upgraded ORKA, along with a potential time reversal violation experiment in the charged mode along with a more general purpose neutral experiment that would measure the $\mathrm{K}_{\mathrm{L}} \rightarrow \pi^{0} 1^{+} 1^{-}$modes.

The $\mathrm{K} \rightarrow \pi v v$ experiments require very high $\pi^{0}$ detection efficiency, with inefficiency well below $10^{-6}$, for $\pi^{0}$ photons of $\sim 10 \mathrm{MeV}$ to $\sim 1 \mathrm{GeV}$. In addition, the $\mathrm{K}^{+} \rightarrow \pi^{+} v v$ experiment needs high light collection from plastic scintillator in a $\sim 1.5 \mathrm{~T}$ magnetic field. The $\mathrm{K}_{\mathrm{L}} \rightarrow \pi^{0} v v$ experiment needs excellent energy, time and directional measurements of the $\pi^{0}$ photons.

\section{Muons}


Rare muon decays provide exceptional probes of flavor violation beyond the Standard Model physics. The observation of charged lepton flavor violation (CLFV) is an unambiguous signal of new physics and muons, because they can be made into intense beams, are the most powerful probe. Experiments at Project X using charged lepton flavor violation can probe mass scales up to $\mathrm{O}\left(10^{4}\right) \mathrm{TeV} / \mathrm{c}^{2}$.

The US experimental program naturally starts with the $\mathrm{g}-2$ and $\mathrm{Mu} 2 \mathrm{e}$ experiments currently under construction, using intense muon campus beams fed by the Fermilab Booster. Confirmation of the current discrepancy of g-2 with the Standard Model or the observation of $\mathrm{Mu} 2 \mathrm{e}$ would require follow-up experiments. A continuation of this program into the Project-X era would likely include muon-to-electron conversion experiments with a range of nuclear stopping targets and next generation experiments to pursue $\mu \rightarrow$ e $\gamma$ and $\mu \rightarrow 3 \mathrm{e}$.

\section{Super Flavor Factories}

Current generation heavy flavor factories $(b, \tau, c)$ have severely constrained physics beyond the Standard Model, notably with discovery and measurement of $\mathrm{B}_{\mathrm{s}} \rightarrow \mu \mu(\mathrm{CMS}, \mathrm{LHCb})$ close to the Standard Model prediction and ever tighter limits on $\tau \rightarrow 3 \mu$ and $\tau \rightarrow \mu \gamma$. (Belle, LHCb). Evolution to Belle-II and the $\mathrm{LHCb}$ upgrade require higher performance lower mass tracking, particle ID, and breakthrough data acquisition performance. The LHCb upgrade represents the first major particle physics experiment with a fully streaming "triggerless" data acquisition system where zero-suppressed data is fully streamed from frontends to high level event processing and filtering. Other next generation experiments including Mu2e and ORKA have fully streaming readout design aspirations as well and will benefit greatly from the $\mathrm{LHCb}$ fully streaming experience and are watching this development closely. Next generation flavor factories are also require and drive the development of radiation hard silicon PMT (SiPM) readout technology which is also broadly applicable to next generation high-rate intensity frontier experiments.

\section{Very High Precision Measurements}

Experimental progress on the intensity frontier is driven by ever increasing sensitivity to rare processes and every increasing precision on measurement of fundamental parameters such as the muon anomalous magnetic moment (g-2), Electric Dipole Moments (EDMs), and Moller electron-electron scattering.

\section{[Integrate with contributions from Lu and KK]}

\section{Calorimetry}

Kaon and muon experiments have challenging electromagnetic calorimetry requirements. The Project-X Physics Study (PXPS) Electromagnetic Calorimetry Working Group investigated a series of muon, kaon and neutron-antineutron oscillation experiments in existing or proposed pre-Project $\mathrm{X}$ versions and in several instances examined whether or not the calorimetric 
techniques employed in these experiments could be extrapolated to produce viable experiments at Project $\mathrm{X}$ in its various stages. In so doing, areas of potentially fruitful R\&D in calorimetry were identified. The resulting initiatives have both short term experiment-specific goals, and longer term more generic objectives. The PXPS calorimetry report is included in Appendix-1 and calorimetry specific recommendations will be further elucidated at the Minnesota Snowmass meeting.

\section{Tracking}

Kaon and muon experiments have challenging tracking requirements. In addition to excellent position resolution, requirements include: extremely high rate capability (potentially up to 1 $\left.\mathrm{MHz} / \mathrm{mm}^{2}\right)$, extremely low mass $\left(<<1 \% \mathrm{X}_{0}\right)$ and in some cases good timing resolution $(<1 \mathrm{~ns})$. The tracking working group of the Project-X Physics Study undertook to survey tracking requirements of potential Project $X$ experiments and the capabilities of available technologies, with the goal of identifying high priority areas for R\&D. The PXPS tracking report is included in Appendix-2 and tracking specific recommendations will be further elucidated at the Minnesota Snowmass meeting.

\section{Data Acquisition}

Next generation experiments will benefit greatly from advances in networking and processing technologies driven by the IT and telecommunication revolution. Network switching technologies are now woven into "fabrics" with fully programmable point-to-point connectivity between front-end data sources and processing resources. Standard protocols such as Ethernet on standard fabrics can sustain 10-100 GByte/second of data volume from front-ends to processing. Forced by power density considerations, modern processing is evolving away from high performance single CPU cores toward a large number of integrated low-power CPU cores. Processing is now also embracing the paradigm of computing "fabrics" which define a new Moore's law of performance. Next generation filter processing must be performed in the context of these high density multi-core fabrics with smaller local cache memory for each computing core, which is a significant evolution challenge for event processing software today. Meeting this challenge is highly motivated by potential gains in sensitivity afforded by the highlevel processing of all events, for example up to a factor of $\mathrm{x} 10$ for complex events in $\mathrm{LHCb}$ and at least a factor of $\mathrm{x} 3$ for ORKA over the previous generation of kaon experiments.

Evolution to the computing fabric model is necessary in order to sustain Moore's Law advances, and necessary to exploit the advances in current network fabric performance. This is a common challenge shared by both "online" and "offline" computing which further motivates development of streaming data acquisition architectures.

\section{Computing and Simulation}


As noted in the data acquisition discussion, progress toward streaming acquisition architectures reliant on high level filter processing requires very close integration with "offline" computing which effectively becomes another species in a high level processing ecosystem. Next generation intensity frontier experiments can expect to reasonably and economically steward 1-10 Peta-Bytes of data thanks to the pioneering efforts of the LHC experiments which steward 10-100 Peta-Bytes of data per experiment. Despite these enormous gains in data storage capability that characterize the "Big Data" era, streaming data acquisition systems for intensity frontier experiments will require filtering rejection of greater than $\mathrm{x} 1000$. Realizing this ecosystem where $>99.9 \%$ of fully reconstructed are rejected forever will require a robust fault tolerant "self-aware" computing framework and associated applications in order to capture the benefit of fully streaming architectures.

Simulation strategies to model rare processes often rely on factorizing the problem in order to, for example, estimates background processes at the 1 per trillion level $\left(10^{12}\right)$. While computing systems now exist to provide the raw horsepower for simulating in excess of one billion $\left(10^{9}\right)$ events, this computing power cannot be exploited without an associated level of integration maturity in the simulation software. The evolution of the GEANT simulation environment is a major success in this regard, yet there are many simulation challenges for future intensity frontier experiments that require further development. Notably a comprehensive simulation of neutrino-nucleus interactions with state-of-the-art generators does not exist within GEANT4 today, requiring researchers to factorize and patch in leading neutrino-interaction generators with GEANT in order to benefit from the high quality particle cascade simulations available in GEANT. Further progress in the treatment of low energy interactions, neutron transport in particular will also be important to high fidelity modeling of rare processes important to intensity frontier research.

\section{Conclusions}

A robust intensity frontier program to fully exploit Project-X beam opportunities requires a robust program of detector $R \& D$. The foremost focus for this $R \& D$ program should be upgrades to the neutrino, kaon and muon programs. A core outline of the experimental program has been discussed above, in which upgrades to LBNE, Mu2e and ORKA will compliment a KOPIO-like experiment and possible Mueg and Mu3e experiment. Given the tremendous power and versatility of the Project-X beams, we should expect other scientific opportunities will arise and in fact, such opportunities will be facilitated by a robust detector R\&D program.

The rough outline of an R\&D program that will enable a world leading onshore US intensity frontier program have been presented above. It is important that these thrusts, synergistic with the intensity frontier program obtain sufficient funding and as the experimental program continues to develop, sufficient R\&D funding will be required from the intensity frontier itself. 



\title{
Appendix-1: Project X Physics Study Calorimetry Report
}

\section{Electromagnetic Calorimetry in Project X Experiments - The Project X Physics Study}

\author{
Summary and R\&D prospects
}

\author{
Fritz DeJongh, Milind Diwan, Corrado Gatto, David Hitlin, David E. Jaffe, Yuri Kamyshkov, \\ Laurie Littenberg, William Molzon, Anna Mazzacane, Andrei Poblaguev, Peter Winter, \\ Elizabeth Worcester, Minfang Yeh, Ren-yuan Zhu
}

\section{Introduction}

The Electromagnetic Calorimetry Working Group investigated a series of muon, kaon and neutron-antineutron oscillation experiments in existing or proposed pre-Project $\mathrm{X}$ versions, and in several instances examined whether or not the calorimetric techniques employed in these experiments could be extrapolated to produce viable experiments at Project $\mathrm{X}$ in its various stages. This was done, as per our charge, purely from the perspective of the calorimeters involved. In so doing, areas of potentially fruitful R\&D in calorimetry were identified. The resulting initiatives have both short term experiment-specific goals, and longer term more generic objectives.

\section{Muon physics}

Experiments examined included an improved measurement of $g-2$ of the muon, a search for $\mu^{-}$to $e^{-}$conversion and related searches for $\mu^{-}$to $e^{+}$conversion and $\mu^{+} \rightarrow 3 e$ decay, and a search for $\mu^{+} \rightarrow e^{+} \gamma$ decay.

\section{$g-2$}

The BNL $g$-2 experiment uses Pb-scintillating fiber electromagnetic calorimeters, read out with PMTs, to detect decay electrons at twenty four stations around the ring. The Fermilab version of the experiment will upgrade these calorimeters to cope with rates as much as three times higher. $\mathrm{PbWO}_{4}$ scintillating crystals, $\mathrm{PbF}_{2}$ Cerenkov counters and $\mathrm{W} /$ scintillating fiber devices have been considered. Arrays of the latter two have been tested in the Fermilab test beam from 2 to $8 \mathrm{GeV}$. The $\mathrm{PbF}_{2}$ crystals are the leading candidate. Readout would be with either Hamamatsu R9800 PMTs or S10362-33-050C sixteen channel SiPMs.

\section{Mu2e}

The $\mathrm{Mu} 2 \mathrm{e}$ configuration contains an electromagnetic calorimeter designed to furnish confirmation of a $105 \mathrm{MeV}$ conversion electron signal from the tracker, as well as to provide a possible trigger. The calorimeter consists of 2000 LYSO crystals, read out by large area APDs. The default geometry is four vanes arrayed around the beamline; an 
alternative disk geometry is also under consideration (see Figure 1). The LYSO has a decay time of $40 \mathrm{~ns}$, requiring a gate interval of about $200 \mathrm{~ns}$. This is sufficient to reject pileup in the current incarnation of the experiment, and perhaps in the early stages of a Project X-capable experiment. It would not suffice for the increases in intensity contemplated at later stages. For this, since energy resolution is of prime importance, a new faster scintillator would have to be employed (see below).
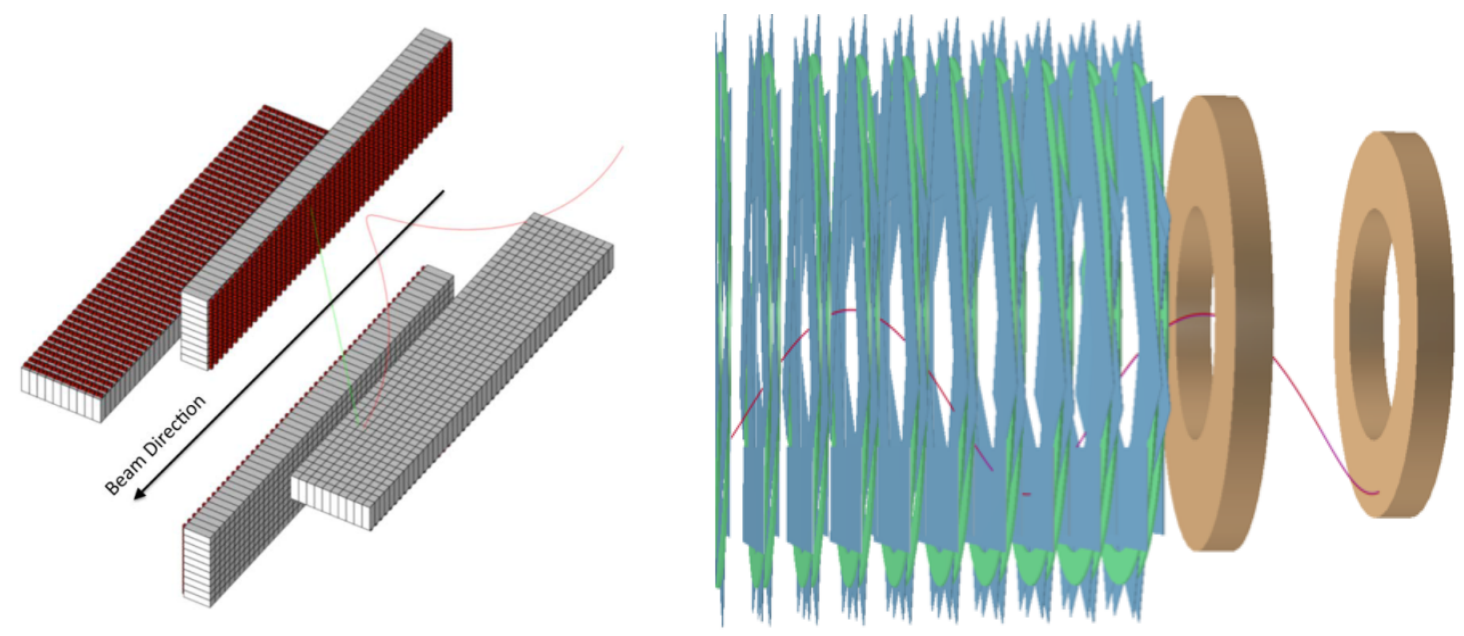

Figure 1. Vane (left) and disk (right) calorimeter configurations in the Mu2e experiment.

$\mu^{+} \rightarrow e^{+} \gamma$

The current MEG experiment at PSI was presented, with an eye to examining the planned upgrade and the possibility of employing a calorimetric technique for a $\mu^{+} \rightarrow e^{+} \gamma$ search at Project X. The current data sets a limit of $2.4 \times 10^{-12}$ at $90 \% \mathrm{CL}$. The liquid xenon calorimeter did not quite reach design specification; upgrades are planned to improve the energy resolution. These involve improving the spatial resolution of the detectors mounted on the entrance face by replacing the existing PMTs with large area $(12 \times 12$ $\mathrm{mm}^{2}$ ) MPPCs, as shown in Figure 2, reducing the reflectivity of the inner walls and improving the time resolution. The expected sensitivity of the experiment would then improve to a few $\times 10^{-13}$.

It is unlikely, however, that the MEG technique can be successfully employed at Project $\mathrm{X}$ rates, since the accidental rate increases as the square of the stopping muon rate. This has motivated a study of an alternative approach, in which the photon is detected by reconstructing the tracks of an $e^{+} e^{-}$pair produced in a thin converter. This amounts to a tradeoff of detection efficiency for energy resolution and concomitant background rejection. Early simulations indicate that this non-calorimetric technique could reach a single event sensitivity of $2 \times 10^{-16}$. The concept uses double pixel layers to measure the 
position and direction at several points on the helical trajectory of a track in a solenoidal magnetic field, as shown in Figure 3.
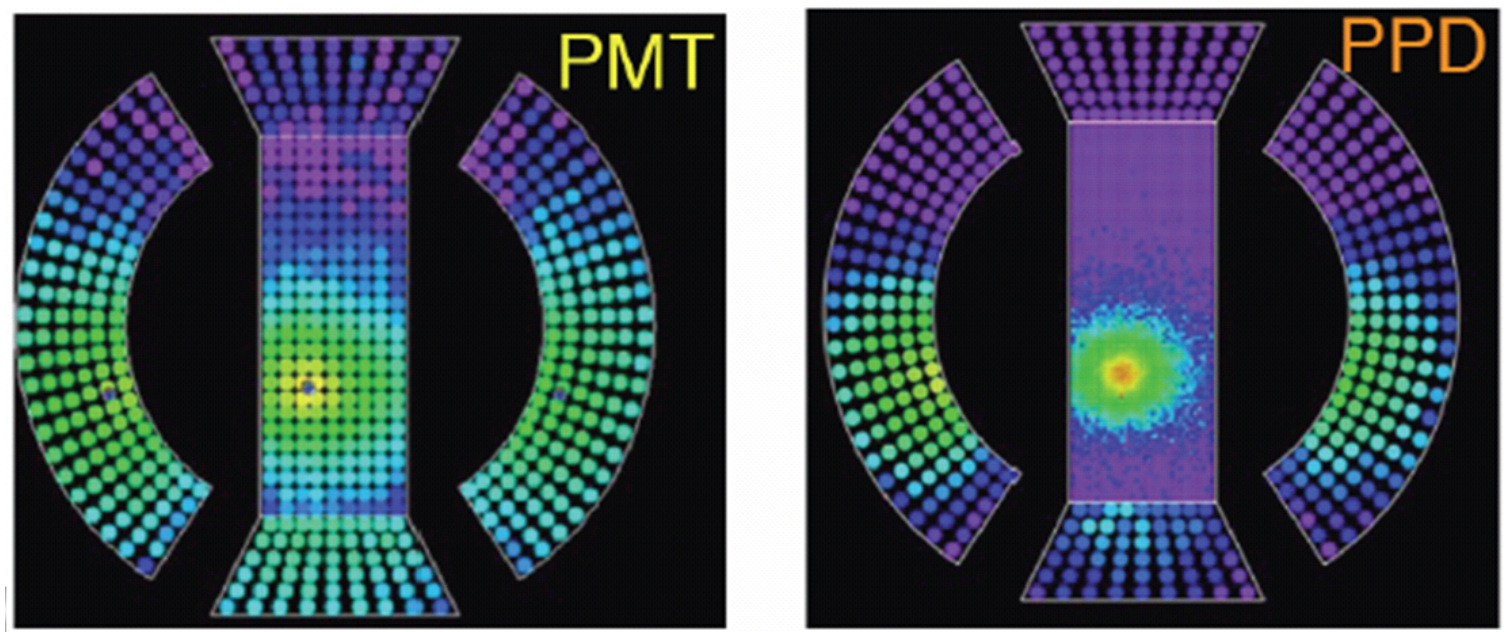

Figure 2. Improvement in shower localization to be achieved by MEG by replacing PMTs on the LXe calorimeter entrance face with MPPC devices and reducing wall reflectivity.

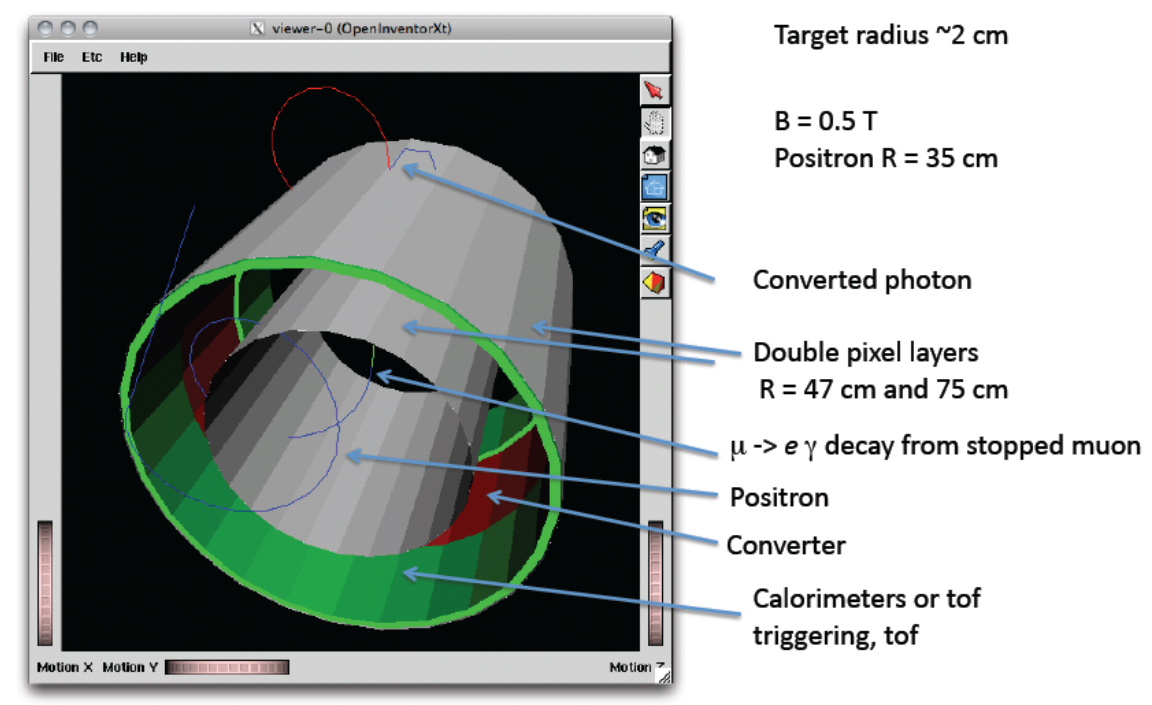

Figure 3. Concept of a $\mu^{+} \rightarrow e^{+} \gamma$ experiment using a photon conversion technique.

The high muon and kaon rates expected at Project $\mathrm{X}$ make high quality calorimetry quite difficult. The candidate technologies are based on homogeneous scintillating devices, either noble liquids or crystals. While LXe has a fast decay time of $45 \mathrm{~ns}$, the large radiation length and practical requirement that the light be detected at the periphery of a large volume, limit the achievable time resolution. The current generation of crystalbased high quality, high rate capable, calorimeters employs $\mathrm{PbWO}_{4}$ and $\mathrm{LYSO}$, which have decay times of 30 and 40 ns, respectively. Project $X$ will increase the rates of 
stopping muon experiments by nearly a factory of fifty, which makes these decay times far too slow to prevent such calorimeters from being overwhelmed by accidentals.

This leads to the question of whether there are other radiation-hard crystals having decay times that are meaningfully faster. $\mathrm{BaF}_{2}$, has the fastest known scintillation component, with a decay time of $600 \mathrm{ps}$ at $220 \mathrm{~nm}$, representing $\sim 15 \%$ of the total light output. Unfortunately, $85 \%$ of the light, peaking at $300 \mathrm{~nm}$, has a decay time of $650 \mathrm{~ns}$. This crystal was studied in some detail as a candidate technology for the GEM detector at the $\mathrm{SSC}$, but has received little attention in the past two decades. Realizing the promise of a high resolution sub-nanosecond electromagnetic calorimeter requires further development in two areas. The first is the properties of the crystal itself. There is evidence that doping $\mathrm{BaF}_{2}$ with lanthanum rather than cerium can reduce the slow component while having little effect on the fast component. This parameter space should be further explored. If a more optimum doping is found, other important properties, such as radiation harness, then need to be measured. The second area is the photodetector. There are solar-blind photocathodes for PMTs that can further enhance the fast signal response over the slow, but suitable devices that have the appropriate spectral response, rise time and magnetic field insensitivity do not yet exist. There are, however, several promising avenues that can be explored. One is to extend the current work at Chicago, Argonne, LBNL and Fermilab on fast, large area planar microchannel plate PMTs to use solar-blind photocathodes such as K-CS-Te. The other is to develop SiPM or APD devices with UV spectral sensitivity. There are small devices with enhanced UV sensitivity; these would have to be extrapolated to larger sizes. This two-prong approach should form the basis of an interesting collaborative R\&D initiative.

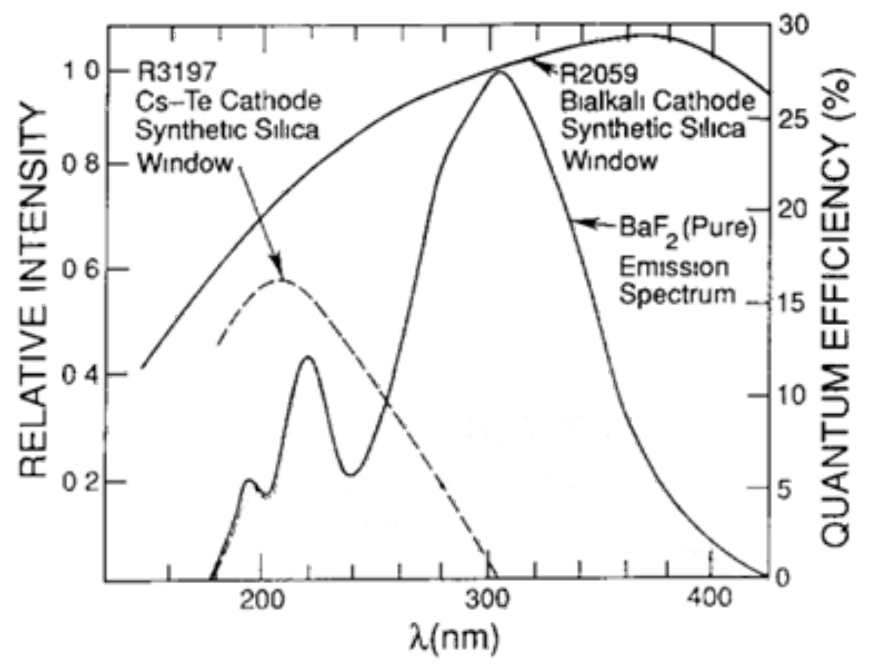

Figure 4. Scintillation light spectrum of $\mathrm{BaF}_{2}$, showing the fast $220 \mathrm{~nm}$ and slow $300 \mathrm{~nm}$ components, along with the spectral response of two types of photocathodes. 


\section{Work needed on muon experiments}

The more than one order-of-magnitude increase in muon stopping rates at Project $\mathrm{X}$ will require new approaches to photon detection. There are two main thrusts:

- Explore the reach of $\mu^{+} \rightarrow e^{+} \gamma$ experiments with the technique of detection of the $e^{+} e^{-}$ pair from photon conversion.

- Extend high quality scintillating crystal calorimetry to the sub nanosecond realm by developing new dopings for crystals such as $\mathrm{BaF}_{2}$, and new fast solar-blind readout devices.

\section{Kaon physics}

Experiments examined included a $K^{+} \rightarrow \pi^{+} v \bar{v}$ decay measurement and a search for the decay $K_{L}^{0} \rightarrow \pi^{0} v \bar{v}$, along with a review of the $\mathrm{KTeV}$ experience.

$K^{+} \rightarrow \pi^{+} v \boldsymbol{v}$

ORKA (see Fig. 5) is a proposed FNAL experiment to measure the branching ratio of $K^{+} \rightarrow \pi^{+} v v$, predicted by the standard model to be $\sim 7 \times 10^{-11}$. The observed final state in $K^{+} \rightarrow \pi^{+} v v$ decay is a single charged pion; the main sources of background are the twobody kaon decays $B\left(K^{+} \rightarrow \pi^{+} \pi^{0}\right)=21 \%$ and $B\left(K^{+} \rightarrow \mu^{+} v\right)=63 \%$. The rejection of $K^{+} \rightarrow \pi^{+} \pi^{0}$ is relevant for the requirements on calorimetry. Assuming a signal-to-background of at least 10 , the $K_{\pi 2}$ decay background must be suppressed by a factor of $\sim 3 \times 10^{10}$ or more. $K_{\pi 2}$ rejection is achieved by accurate reconstruction of the kinematics of the charged pion and detection of the photons from $\pi^{0}$ decay. To reduce background from $K_{\mu 2}$ and other muon background, the final state decay chain $\pi^{+} \rightarrow \mu^{+} \rightarrow e^{+}$is reconstructed in the drift chamber and range stack. The range stack introduces almost one full radiation length of material between the decay point and the calorimeter.

The measurement of $B\left(K^{+} \rightarrow \pi^{+} v v\right)$ in a stopped-kaon experiment requires detection of photons in the energy range (20-225) MeV from $\pi^{0}$ decay. E949 successfully observed $K^{+} \rightarrow \pi^{+} v v$ with a rejection of $\sim 10^{6}$.

High photon veto efficiency requires conversion and detection of photons. Photon detection can be maximized by a fully active, hermetic calorimeter with sufficient thickness in radiation lengths $\left(X_{0}\right)$. Studies for the KOPIO experiment indicate that $23 X_{0}$ would be sufficient for a stopped-kaon experiment. Inoperative calorimeter elements imply a loss of hermiticity, so redundancy or a high degree of single-channel reliability is required. The stopped-kaon experiment requires a solenoidal magnetic field and the calorimeter must be accommodated by the solenoid. This constraint may imply a compromise between the fully active and thickness requirements. Inactive components of 
the calorimeter, such as lead layers, effectively lower the minimum photon energy threshold from $20 \mathrm{MeV}$.

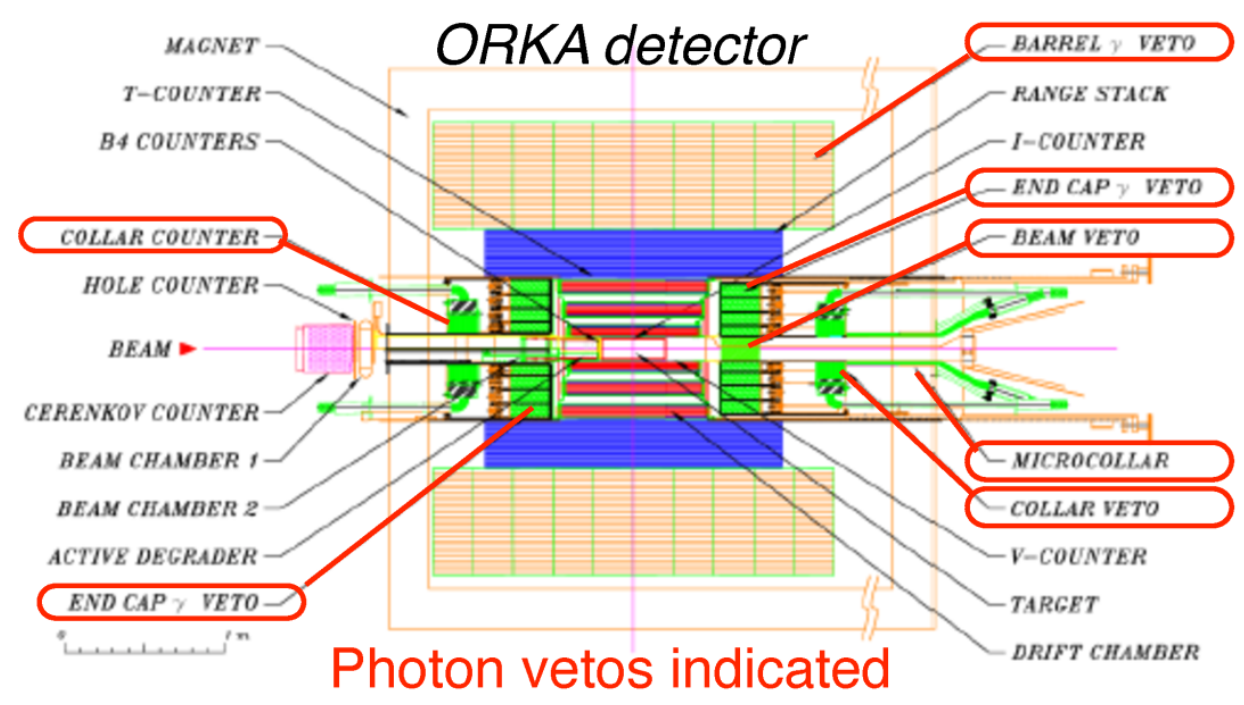

Figure 5. Elevation view of the proposed ORKA detector indicating the locations of photon veto detectors. The beam enters from the left.

High photon veto efficiency must be achieved while minimizing acceptance losses due to vetoing on random or 'accidental' detector activity. These acceptance losses can be minimized by minimizing the time interval required for the coincidence of $K^{+}$decay and the putative photon.

A scintillator-based calorimeter would need to have a short decay time, high light yield, and high collection efficiency to satisfy the veto requirement. The ability to reconstruct photons and electrons for other (non- $K^{+} \rightarrow \pi^{+} v v$ ) measurements in the experiment would place additional requirements on the calorimeter energy, position, and shower timing resolutions. Improvements to the reconstruction ability of the calorimeter may also reduce systematic uncertainties for $K^{+} \rightarrow \pi^{+} v v$; for example, $\pi^{0}$ reconstruction would allow for studies of $\pi^{+}$detection efficiency using $K^{+} \rightarrow \pi^{+} \pi^{0}$ decays. Studies to quantify the size of these potential improvements would be helpful in choosing among design options.

As proposed, ORKA has two primary calorimetric elements: the endcap and the barrel veto. For the endcap, ORKA could re-use the $\sim 20025 \mathrm{~cm}$ long CsI crystals from BNL E949 that provide $13.5 X_{0}$ total depth. The expected energy and timing resolutions are $\Delta E / E \sim 11 \%$ and $\Delta t \sim 0.7 \mathrm{~ns}$, respectively. Use of shashlyk detectors, with greater depth, is also a possibility that should be explored.

Several designs are under consideration for the barrel veto. A shashlyk style calorimeter similar to the one designed for KOPIO (see Sect. 0.1.2 below) would satisfy the photon 
veto requirement. A possible configuration is 155 interleaved layers of $0.8-\mathrm{mm}$ lead and 1.6-mm scintillator read out by $\sim 400$ wavelength shifting fibers. This provides $23 X_{0}$ total depth. The expected energy and timing resolutions are (3-4)\%/ $\sqrt{E}$ and (90-100) $\mathrm{ps} / \sqrt{ } E$, respectively. The noise term in the energy resolution is negligible above $50 \mathrm{MeV}$ so was not included in the parameterization for KOPIO, but will likely be relevant for ORKA because the photon energies will be lower. A shashlyk calorimeter has already been prototyped for KOPIO. For ORKA, because it must fit inside the CDF solenoid, the detector must be somewhat more compact without losing veto power. More work on design and prototyping to fulfill this size requirement is needed.

Another option for the calorimeter design is a totally-active ADRIANO calorimeter consisting of 150 interleaved layers of $2 \mathrm{~mm}$ lead glass and $2 \mathrm{~mm}$ fast scintillator. Work to simulate the performance of this style detector in ORKA is ongoing, but the energy resolution is expected to be better than a shashlyk style detector, particularly at low energies. It may also allow reconstruction of angles using timing or light division methods. From the perspective of ORKA, the most important benefit of the totally-active ADRIANO calorimeter would be the potential reduction in photon inefficiency relative to a shashlyk style calorimeter.

NA62 at CERN is a decay-in-flight experiment, currently under construction, to measure $B\left(K^{+} \rightarrow \pi^{+} v v\right)$. The technique will be complementary to that of ORKA because decay-inflight and stopped-kaon experiments are sensitive to different parts of phase space.

$K_{L}^{0} \rightarrow \pi^{0} v \bar{v}$

KOPIO (see Fig. 2) was a proposed BNL experiment to measure the branching ratio of $K_{L}^{0} \rightarrow \pi^{0} v \bar{v}$. It is expected that a similar experiment will be proposed at FNAL in the Project X era. The standard model value of the $K_{L}^{0} \rightarrow \pi^{0} v \bar{v}$ branching ratio is $\sim 3 \times 10^{-11}$.

$K_{L}^{0} \rightarrow \pi^{0} \pi^{0}$ is the main background. The two-photon final state of the signal requires accurate reconstruction of single photon energy, position, direction and time. The photon veto requirements are more stringent than $K^{+} \rightarrow \pi^{+} v v$ (Section 0.1.1) because background photon energies can extend below $20 \mathrm{MeV}$ due to decay-in-flight of the $K_{L}^{0}$. Also in contrast to a stopped-kaon experiment, the beam represents an extended signal and background source that alters the hermeticity requirements. The minimum performance requirements are:

- Direction resolution: $25 \mathrm{mrad} @ 250 \mathrm{MeV}$

- Energy resolution: $3 \% / \sqrt{ } E$

- Timing resolution: $\sim 100 \mathrm{ps} / \sqrt{ } E$

- Position resolution: $250 \mu \mathrm{m}$

- Veto efficiency: $10^{-4} / \gamma$ 


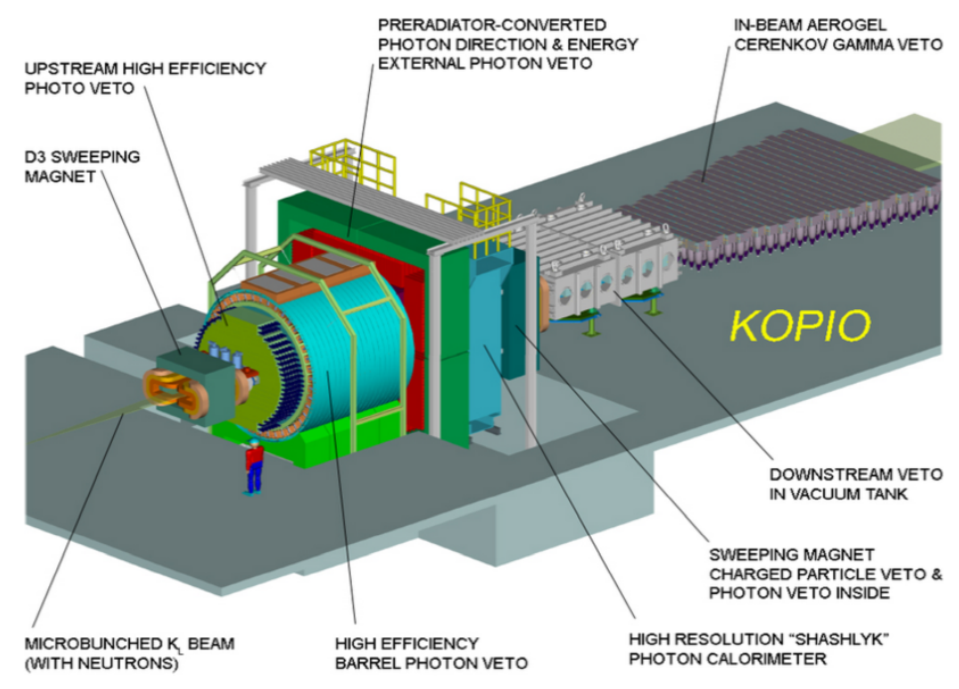

Figure 6. Schematic of the proposed KOPIO detector. The pre-radiator is shown in green and the shashlyk calorimeter is shown in blue.

The measurement of photon energies and directions in KOPIO is made by the combination of a pre-radiator and a shashlyk style calorimeter. The pre-radiator consists of scintillator sheets alternating with cathode strip drift chambers. It contains $2.7 X_{0}$ of material in total; it is important to keep the thickness in the beam direction of the preradiator as small as possible in order to limit transverse shower size so that photons from background decay modes may be resolved. The result of this configuration is that most photon interactions are $e+e^{-}$pair production in which the angular separation of the $e+e^{-}$ pair is small enough that the individual particles need not be resolved by the calorimeter. The initial direction of $e+e^{-}$pair is the same as that of the photon, so the track reconstructed in the pre-radiator drift chambers is a measurement of the photon angle. Following the pre-radiator is a $19 X_{0}$ shashlyk style calorimeter (lead-scintillator sandwiches read out by wavelength shifting fibers passing through holes in the scintillator and lead), with transverse granularity of $\sim 10 \mathrm{~cm}$. By design, the KOPIO preradiator/calorimeter combination satisfies the performance specifications above.

A study comparing the improvement in signal yield relative to background for various improvements in calorimeter performance finds that improvements to angular and energy resolution are most beneficial. Reduction of photon veto inefficiency also provides significant improvement in S/B. Timing resolution is less critical, and position resolution least significant.

Studies from KOPIO show that energy resolution improves as the thickness of the lead plates is reduced, the thickness of the scintillator plates is increased, and as the number of layers increases. These design parameters correspond to increased sampling efficiency 
and increased photostatistics, which are two of the three primary drivers of the energy resolution. The third is light collection uniformity, which can be improved by increasing the light collection efficiency near the edges of a scintillator tile.

Angular resolution might be improved if the pre-radiator could resolve the opening angle between the $\mathrm{e}^{+} \mathrm{e}^{-}$pair; this would require use of a high-speed, high-resolution tracker or TPC that does not add large amounts of material to the detector. The ability to reconstruct angles using the calorimeter would also be helpful. These ideas are speculative and have not yet been studied.

KOPIO achieved a modest increase in signal acceptance by using the barrel photon veto (see Fig. 6). Improvements in angle, position, and energy resolution of calorimetry would boost this acceptance.

KOTO is an experiment at J-PARC to search for $K_{L}^{0} \rightarrow \pi^{0} \pi^{0}$ decay, building on its predecessor, E391 at KEK. It is currently under construction and expects to begin taking data in 2013. Calorimeter and photon veto performance are critical to this experiment; one of the major upgrades between E391 and KOTO is the use of the KTeV CsI crystals for the KOTO calorimeter. Experience from this experiment will be very useful in the design of a KOPIO-style experiment at Project X.

\section{Work needed on kaon experiments}

The calorimeters needed for kaon experiments in the Project X era are reasonably well understood; the shashlyk style calorimeter designed for KOPIO has been prototyped and likely can be modified to meet the design requirements for both ORKA and a KOPIOstyle experiment. However, it may be possible to significantly improve the sensitivity of these experiments and/or broaden their physics reach by making improvements to the calorimetry. Much work remains to optimize the calorimeter design and study which improvements to the calorimeter are most likely to pay off in improvements to the measurements. Finally, R\&D in 3D calorimetry and low energy photon veto detection would be of general use to the HEP community. Specific studies that are needed include:

- Compact shashlyk design for ORKA: the ORKA calorimeter must fit inside the CDF solenoid. The shashlyk calorimeter designed for KOPIO must be made more compact without sacrificing veto efficiency.

- Benefits to ORKA from improved calorimetry: the size of potential improvements to the measurement of $K^{+} \rightarrow \pi^{+} v v$ resulting from improvements to calorimeter resolution should be quantified.

- Benefits of ADRIANO calorimeter: the size of potential improvements to the measurement of $K^{+} \rightarrow \pi^{+} v v$ resulting from improved photon veto efficiency in a totallyactive calorimeter should be quantified. 
- KOPIO calorimeter optimization: improvements to the energy and angular resolution of the KOPIO calorimeter result in significant improvements to the measurement of $\mathrm{K}_{\mathrm{L}}^{0} \rightarrow \pi^{0} v v$. The detector should be optimized to improve the energy resolution as much as possible. Some thought should be given to potential ways to improve the angular resolution.

- 3D calorimetry: The ability to "track" photons while maintaining high photon veto efficiency is highly desirable. Development of 3D calorimetry techniques such as time difference methods and light division methods as well as R\&D for calorimeters with $z$ segmentation, with a focus on preservation of veto efficiency, would be useful for $K \rightarrow \pi v v$ experiments.

- Low energy photon veto detectors: This document has primarily focused on scintillation detectors. Generic R\&D for low energy photon veto detection is important. New design ideas that improve photon veto efficiency could significantly improve the sensitivity of $K \rightarrow \pi v v$ experiments. 


\section{Appendix-2: Project X Physics Study Tracking Report}

\section{Tracking Working Group Summary}

Co-conveners: Ron Lipton and Jack Ritchie

\section{Introduction}

Kaon and muon experiments at Project $\mathrm{X}$ have challenging tracking requirements. In addition to excellent position resolution, requirements will include: extremely high rate capability (potentially up to $\left.1 \mathrm{MHz} / \mathrm{mm}^{2}\right)$, extremely low mass $\left(<<1 \% \mathrm{X}_{0}\right)$ and in some cases good timing resolution $(<1 \mathrm{~ns})$. This working group undertook to survey tracking requirements of potential Project X experiments and the capabilities of available technologies, with the goal of identifying high priority areas for $R \& D$.

The activities of this working group consisted of presentations in parallel sessions that were then followed by scheduled discussion sessions on subsequent days. The parallel sessions were divided approximately equally between silicon tracking and gaseous detectors. Attendance of the parallel sessions was typically about 15 persons. The discussion sessions had lower attendance, at least in part because some of the presenters were no longer present at Fermilab.

A list of the parallel session presentations follows:

Session 1 (Saturday June 16 morning)

- Tracking Requirements in Rare K Decay Experiments - Jack Ritchie

- Mu2e Tracker - Aseet Mukherjee

- Straw Tracking for g-2 - Hogan Nguyen

Session 2 (Saturday June 16 afternoon)

- Low Mass, High Speed Silicon Tracking - Ron Lipton

- The NA62 Gigatracker - Bob Velghe

Session 3 (Monday June 18 morning)

- The ORKA Drift Chamber - Toshio Numao

- Multi-anode Straws - Seog Oh

- Silicon Tracker for $\mu$ e - Fritz DeJongh

Session 4 (Monday June 18 afternoon)

- Tracking for nnbar - Mike Snow

- Low-mass Tracker Mechanics - Bill Cooper

- Low-mass Monolithic Active Pixel Detector for STAR at RHIC - Leo Griener

\section{Benchmark Experiments}

Processes that impose challenging tracking requirements on future (and in some cases current) experiments include decay modes such as $\mathrm{K}^{+} \rightarrow \pi^{+} v v, \mathrm{~K}_{\mathrm{L}} \rightarrow \mu \mathrm{e}, \mathrm{K}_{\mathrm{L}} \rightarrow \pi^{0} \mathrm{ee}, \mu \quad \mathrm{e}$, and eee. These decays provide benchmarks that are useful in identifying the requirements that can be expected for Project X rare process experiments. 
Time available during this workshop did not permit new studies to be carried out. Reviewing previous experiments and also on-going experiments can provide some guidance. For instance, the proposed ORKA experiment has a conceptual design for a very low-mass drift chamber, based on a chamber built for previous experiments at the Brookhaven AGS. The NA62 experiment, under construction at CERN, provides an interesting point of reference. The NA62 Gigatracker has very aggressive goals for providing good resolutions in both position and time using a low-mass silicon pixel detector. The experience gained from NA62 will be important for future experiments. NA62 and the Fermilab mu2e experiment illustrate the need for some detectors to operate in vacuum. In both cases, straw tracking is the chosen technology.

While some conclusions can be drawn by looking at past and current experiments, determining the tracking requirements of experiments at Project $\mathrm{X}$ will require detailed simulations that include realistic beam assumptions and specific detector geometries. None of this work has been done. Therefore, one clear priority is to begin work on such simulations.

\section{Low-mass Silicon Tracking}

Silicon tracking detectors can provide micron-level precision and excellent time resolution. This combination will be needed in experiments like $\mu \rightarrow$ e $\gamma$ or NA62, where background rejection depends on precise track reconstruction and accurate timing. NA62 reported time resolution below $200 \mathrm{ps}$ in this workshop and the SLAC/JLAB heavy photon experiment has achieved 2ns using multiple sampling with standard 300 micron thick silicon detectors read out with the CMS APV chip. However combining excellent time and position resolution with low mass and low power is a challenge. The Heavy Flavor Tracker (HFT) group is producing a CMOS MAPSbased vertex detector for STAR at RHIC which is designed to achieve better than 5 micron resolution in a detector thinned to 50 microns with a readout time of $\sim 100$ microseconds. The ladders are air cooled and have a total radiation length of less than $0.3 \%$ per ladder. The time resolution is limited by charge collection by diffusion rather than drift and is zero suppressed but does not utilize sparse readout.

Standard fully depleted silicon detectors analog power requirements are set by the analog power needed to achieve a given time resolution and signal/noise. This reflects directly in the mass budget for power delivery, cooling, and services. The time resolution can be approximated by $\sigma_{t}=t_{\text {rise }} /$ (Signal/Noise). Risetime and noise are in turn directly dependent on the current in the front-end amplifier. This sets fundamental limitations on power needed to achieve the needed time resolution for a given detector capacitance. In the workshop we explored these limits, as well as possible new technologies, such as silicon avalanche diodes integrated in two 3D layers to provide internal amplification (as in a gaseous detector) in combination with a 2 layer coincidence to defeat noise.

Low mass tracking also requires low mass supporting structures and cabling. This is an area of

active work in the collider community. Examples of such structures from collider detector R\&D, 
such as the low mass ladders for STAR, and carbon foam structures being studied by the PLUME collaboration were presented. Work from the SID ILC detector concept on techniques to build low mass systems utilizing high modulus carbon fiber was also presented. Another important area is improvement in the efficiency and mass burden of power delivery utilizing DCDC converters, serial powering, and aluminum cables.

\section{Gaseous Trackers}

Gaseous tracking is a well-developed technology that has been used for many years. In many situations it continues to provide the best tracking solution, particularly when large volumes or large areas need to be covered. The requirements of different experiments can be quite different, so generalizations are difficult. For instance, a somewhat convention cylindrical drift chamber apparently meets the needs in the case of stopped $\mathrm{K}$ experiments, while straws are a more natural solution in decay-in-flight experiments. Also, straws provide a means to instrument an evacuated volume with large-area tracking detectors as in NA62 and mu2e.

The ORKA experiment, proposed to measure $\mathrm{K}^{+} \rightarrow \pi^{+} v v$, needs to achieve the best possible momentum resolution for the $205 \mathrm{MeV} / \mathrm{c} \pi^{+}$from the $\mathrm{K}^{+} \rightarrow \pi^{+} \pi^{0}$ decay, the main background source. The conceptual design for the drift chamber follows the "ultra-thin" design of the BNL E949 chamber. There are a number of open issues concerning possible improvements, such as the optimal cell size and shape, choice of gas, etc. It is also important to achieve good $z$ coordinate resolution, which was achieved in E949 with a separate outer straw tracker (using timing). For ORKA the trade-offs between different alternatives for an outer tracker need to be explored. Also, the need to minimize dead material inside the hermetic photon veto system leads to the novel ideal that possibly the drift chamber endcap could be an active material. In view of the timescale proposed for ORKA, the emphasis needs to be on optimizing the design rather than generic R\&D.

Straws have proven to be a robust and capable technology in a number of experiments. The mu2e system illustrates the strengths of straws: the ability to economically cover a large area with reasonably good resolution and - critically - to operate inside a vacuum region. A similar application is part of the NA62 experiment. The New g-2 Experiment is also exploiting the ability of straws to operate in vacuum by instrumenting two of its calorimeter stations with straw tracking. Tracking information will enable studies of the beam profile and moments, pileup, and calorimeter stability, which will improve the understanding of the systematic errors in the measurement.

The material in a straw tracker is dominated the walls of the straws themselves. While planar geometries are natural for straws, in a cylindrical geometry it is mechanically complicated to include a stereo layer. An interesting idea that addresses both issues is multi-anode (i.e., multiwire) straws. A prototype has been constructed at Duke University that has 12 sense wires per straw, providing an average of about three hits per straw for through-going tracks. Thus, the 
mass per hit is reduced by a significant factor. It also has been shown possible to rotate the endpieces of the straws by 15 degrees to achieve a stereo arrangement of wires within the straws. Tracking with multi-anode straws represents a new idea for an otherwise stable technology and appears to be a favorable direction for R\&D.

It would have been good to include consideration of other gaseous tracking technologies (e.g., GEM, MicroMega, ...), but we were not able to do so during the June workshop.

\section{Conclusions}

Rare decay experiments at Project X are likely to require unprecedented performance from tracking detectors. Each experiment will have its own unique set of requirements. Simulation efforts are needed to define these requirements. Nonetheless, the general themes of low mass and excellent timing are shared among many concepts. In some cases the required performance may be achieved by extending and improving current generations of detectors, such as straw tubes and drift chambers. In others, new ideas are needed to circumvent fundamental limits in existing technologies. This workshop was a first step in exploring and extending the toolkit of detector technology to Project X. 


\section{The LHCb Upgrade}

Physics Driver: The LHCb experiment is

Time Frame: short-intermediate (2018 -2025)

Physics Justification: A strong component of the LHCb Upgrade physics program is the search for new physics signatures in beauty and charm decays. The physics program of the LHCb upgrade extends beyond flavor physics, however, with excellent potential in many other important areas, for example: electroweak physics, selected studies of top physics, generic searches for exotic particles with displaced vertices, and exotic hadron spectroscopy.

Technical limitations: The driving principle of the LHCb upgrade is the implementation of data readout at $40 \mathrm{MHz}$ and a very flexible software based trigger. This will allow the experiment to increase its sensitivity by an order of magnitude for a broad spectrum of final states. In addition several detector subsystems will be upgraded to cope with the higher data rate and to facilitate a better integration with the trigger strategy. High precision vertexing and tracking detectors are being developed as well as novel hadron identification technologies.

Technical capabilities: 1) $40 \mathrm{MHz}$ data acquisition systems, 2) software trigger implementing real time event reconstruction 3) silicon detectors suitable for nonuniform irradiation levels. 4) low mass (cooling and support) tracking system devices, 5) power distribution with noise immunity 6) novel photon detectors with fast timing readout.

\section{Key R\&D Directions:}

1) New ASIC development for $40 \mathrm{MHz}$ silicon readout, 2) new DAQ technologies for $40 \mathrm{MHz}$ readout, 3) 55x55 um pixel cell hybrid pixel devices 4) micro-channel cooling, 5) low mass support system for Si strip detector trackers, 6) low mass cooling for Si strip detector trackers, 7) radiation hard scintillating-fiber and SiPM 8) development of distributed computing and workload management system for high rate throughput 


\title{
PXPS12: 2012 Project X Physics Study: Neutrino Detectors Working Group Summary
}

\author{
Conveners: K. McFarland, R. Tayloe, J. Yoo \\ Contributors: K. Scholberg, J. Spitz, H. Ray, R. Imlay, J. Park, M, Yeh, F. Cavanna, F. DeJongh
}

July 16, 2012

\begin{abstract}
We summarize here the activities of the Neutrino detector working group during the 1423 June, 2012 Project X Physics Study (PXPS12). In addition, we describe a roadmap for related conceptual design activities and detector $\mathrm{R} \& \mathrm{D}$.
\end{abstract}

\section{Overview}

During the 14-23 June, 2012 Project X Physics Study (PXPS12) held at Fermilab, the Neutrino detectors working group coordinated closely with the Neutrino experiments working group and focused on non-oscillation neutrino physics, searches for beyond-Standard-Model effects, and the detector technologies required for neutrino physics in the Project-X era.

There was significant overlap with other working groups and many workshop sessions were held jointly There were approximately 12 speakers invited by this working group on the topics mentioned above. In the next two sections we summarize the physics and associated equipment detector ideas presented by the speakers and discussed among the participants. In the final section, we offer a roadmap for groundwork required to be ready to do experiments for the various Project X stages.

\section{Physics}

The physics addressed by this working group consists of supernovae detection and "neutrinointeraction" physics, categorized by the energy of the incident neutrino. As a separate topic, we discussed $\nu-e$ elastic scattering which is relevant for flux normalization, searches for a neutrino magnetic moment, and a measurement of $\sin ^{2} \theta_{\mathrm{w}}$. The final physics topic addressed was searching for dark sector particles via an intense proton source.

\subsection{Low-energy neutrino physics}

"Low-energy" neutrino physics as categorized here is that done with $E_{\nu} \approx 50 \mathrm{MeV}$ as may be produced with a pion decay-at-rest (DAR) beam. Project X, with the construction of MW-class 1 and $3 \mathrm{GeV}$ proton linacs and an appropriate production target, can contribute significantly.

\subsubsection{Supernova neutrino physics}

There are two possible intersections of supernova neutrinos and Project X: first, assuming that they can be sited underground, most of the large detectors envisioned as a targets for long-baseline 
neutrino beams offer also excellent prospects for the detection of supernova neutrinos. Second, pion DAR neutrino source built as part of a Project X program would offer excellent opportunities for measurement of neutrino-nucleus interaction cross sections relevant for supernova neutrino physics.

A core collapse supernova will produce a burst of neutrinos of all flavors with energies in the few tens of $\mathrm{MeV}$ range. Because of their weak interactions, the neutrinos are able to escape on a timescale of a few tens of seconds after core collapse (the promptness enabling a supernova early warning for astronomers). An initial sharp "neutronization burst" of $\nu_{e}$ (representing about $1 \%$ of the total signal) is expected at the outset, from $p+e^{-} \rightarrow n+\nu_{e}$. Subsequent neutrino flux comes from NC $\nu \bar{\nu}$ pair production. Electron neutrinos have the most interactions with the proto-neutron star core; $\bar{\nu}_{e}$ have fewer, because neutrons dominate in the core; $\nu_{\mu}$ and $\nu_{\tau}$ have yet fewer, since NC interactions dominate for these. The fewer the interactions, the deeper inside the proto-neutron star the neutrinos decouple; and the deeper, the hotter. So one expects generally a flavor-energy hierarchy, $\left\langle E_{\nu_{\mu, \tau}}\right\rangle>\left\langle E_{\bar{\nu}_{e}}\right\rangle>\left\langle E_{\nu_{e}}\right\rangle$ (see Fig. 1).

Despite enormous recent progress, much about the physics of core collapse is not well understood. The neutrino messengers from deep inside the supernova will help us understand many aspects of the supernova mechanism and associated phenomena. The neutrinos are probably intimately involved with the explosion mechanism; imprinted on the flux will be signatures of shock waves, accretion, cooling, possible formation of exotic matter, and further collapse to a black hole, and an improved understanding of supernova nucleosynthesis will result from a detection. The supernova neutrino fluxes will also bring information about the physics of neutrinos themselves. Neutrino oscillations can strongly affect the neutrino fluxes. Matter oscillation effects that come into play as the neutrinos traverse dense stellar matter leave imprints on the spectra.
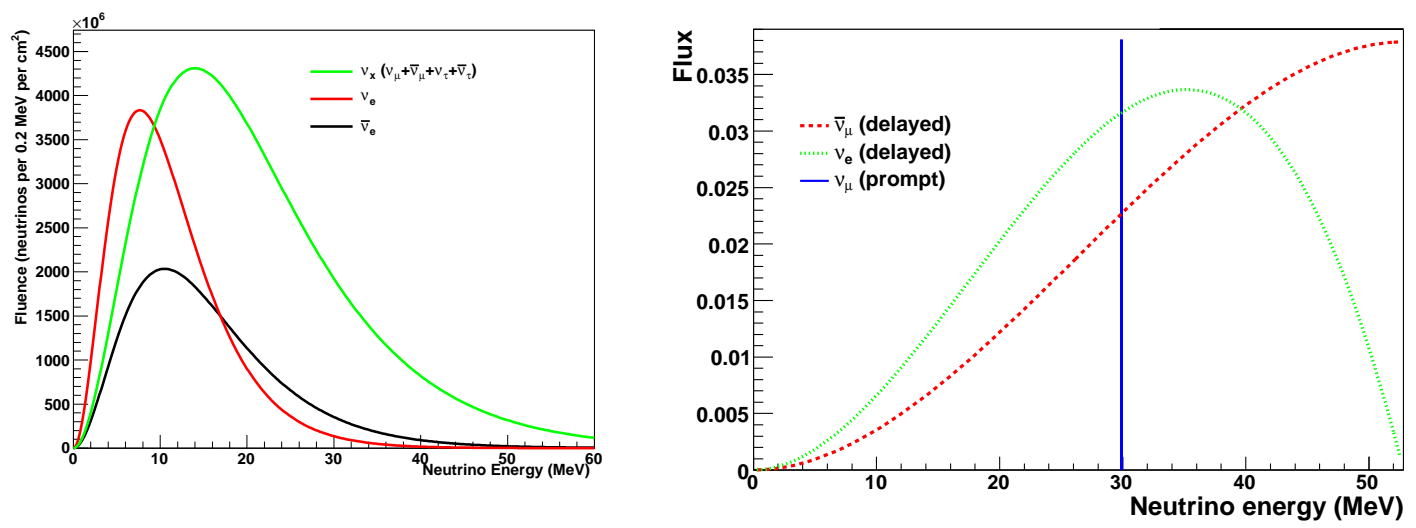

Figure 1: Left: Typical supernova spectrum. Right: Stopped-pion neutrino spectrum.

A stopped-pion source provides monochromatic $30 \mathrm{MeV} \nu_{\mu}$ from pion decay at rest, followed on a $2.2 \mu \mathrm{s}$ timescale by $\bar{\nu}_{\mu}$ and $\nu_{e}$ with a few tens of $\mathrm{MeV}$ from $\mu$ decay. The $\nu$ spectrum matches the expected supernova spectrum reasonably well (see Fig. 1). A $\sim \mathrm{GeV}$, high-intensity, short-pulsewidth, high-duty-cycle proton beam is desirable for creating such a $\nu$ source. Prior examples used for neutrino physics include LANSCE and ISIS.

A rich program of physics is possible with such a stopped-pion $\nu$ source, including measurement of $\nu$-nucleus cross sections in the few tens of $\mathrm{MeV}$ range in a variety of targets relevant for supernova neutrino physics. This territory is almost completely unexplored: so far only ${ }^{12} \mathrm{C}$ has been measured at the $10 \%$ level. Understanding of $\nu$-nucleus interactions in this regime is vital for understanding of supernovae: core-collapse dynamics and supernova nucleosynthesis are highly sensitive to $\nu$ 
processes.

\subsubsection{Coherent neutrino nucleus elastic scattering}

An intense beam of $\mathrm{GeV}$-scale protons provides an immense opportunity for kaon, pion, and muon DAR experiments. Such a source would allow the first detection of and subsequent high statistics sampling of coherent neutrino nucleus scattering events. Although the process is well predicted by the standard model and has a comparatively large cross section $\left(10^{-39} \mathrm{~cm}^{2}\right)$ in the relevant energy region $(0 \sim 50 \mathrm{MeV})$, neutral current coherent scattering has never been observed before as the low energy nuclear recoil signature is difficult to observe.

A modest sample of a few hundred events collected with a keV-scale-sensitive dark matter style detector could improve upon existing non standard neutrino interaction parameter sensitivities by an order of magnitude or more. A deviation from the $\sim 5 \%$ predicted cross section could be an indication of new physics. Either way, the cross section is relevant for understanding the evolution of core collapse supernovae as well as characterizing future burst supernova neutrino events collected with terrestrial detectors.

Well-defined neutrino sources are an essential component to measure coherent-NCAs. There is a unique R\&D opportunity to utilize the $8 \mathrm{GeV}$ proton source. According to a recent MC study, the Fermilab Booster Neutrino Beam is potentially a very good source of DAR neutrinos at the far off-axis $\left(>45^{\circ}\right)$ of the beamline. At the far-off-axis area $\left(>45^{\circ}\right)$, the detector can be placed close enough to the target to gain an inverse-distance-squared increase of the neutrino flux. The pulsed structure of the neutrino beam leads to a substantial advantage in background reduction $\left(1 \times 10^{-5}\right)$ against steady-state cosmogenic and radiogenic backgrounds.

\subsection{Intermediate-energy neutrino physics}

Our classification of "intermediate-energy" neutrino physics consists of measurements using neutrinos of energy $0.1 \lesssim E_{\nu} \lesssim 2.0 \mathrm{GeV}$ such as those available from a pion DIF beam as exists now at Fermilab from the $8 \mathrm{GeV}$ Booster source or the $120 \mathrm{GeV}$ NuMI source. Project X can enable these measurements by providing more POT to these existing neutrino production sources or a new DIF source may be constructed using $8,60,120$, and (perhaps) $3 \mathrm{GeV}$ intense proton beams.

This area of neutrino physics has been pushed along in the last 10 years by the MiniBooNE, NoMAD, and near-K2K experiments. Ongoing investigations are being performed by MiniBooNE, MINERvA, and T2K. Future work will be required to improve the precision of the results and resolve issues that may be inaccessible with the current experiments. It is important to note that understanding this physics is important both for the fundamental physics it illuminates as well as the required quantities it provides to the neutrino oscillation program.

One of the most notable results coming from this work was the observation that the measured neutrino cross sections on light nuclei (carbon, oxygen) for both charged- and neutral-current processes at $E_{\nu} \approx 1 \mathrm{GeV}$ are significantly larger that expected from the state-of-the-art models circa 2000. This was first hinted at in K2K experiment and measured more thoroughly with MiniBooNE. The situation can be seen in Fig. 2 where the data published by MiniBooNE as significantly higher than a model guided by data collected on nucleon targets. That model is also consistent with the NOMAD experiment at higher energies.

The current view of the community is that multinucleon effects within carbon is responsible for this excess in cross section above single nucleon predictions. This will be checked by MINERvA in near future, however if nuclear effects are indeed responsible, a systematic program of measurements into the Project X era will be required to understand the physics across energy and various nuclei. 


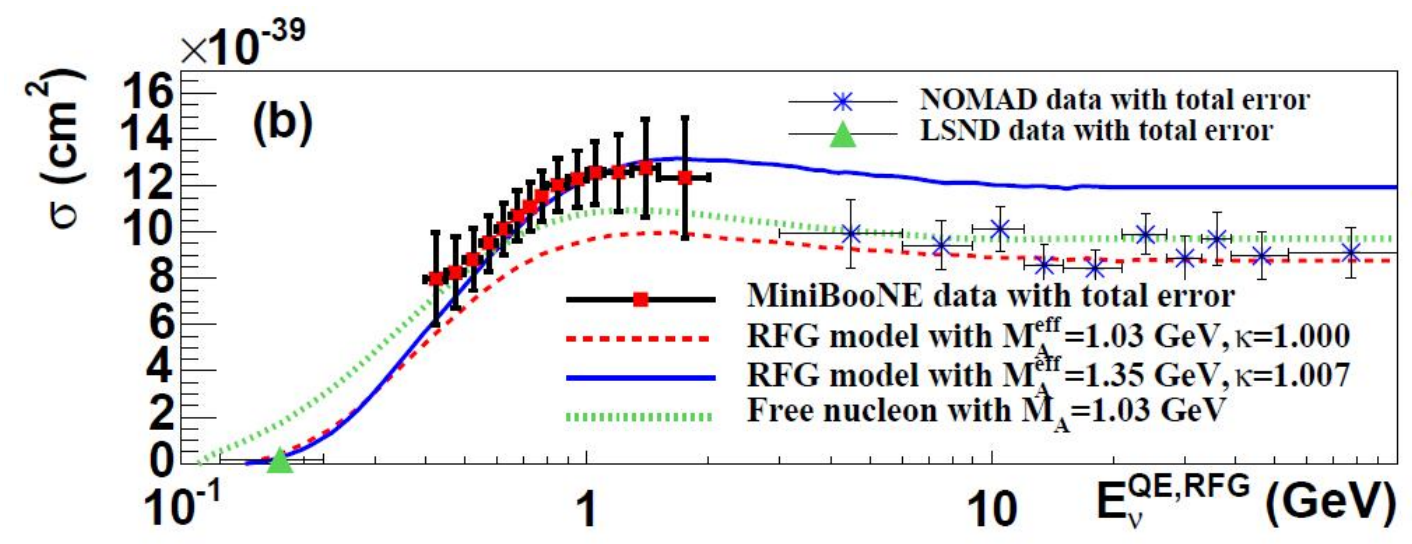

Figure 2: Neutrino-Carbon charged-current quasielastic scattering cross section measured as a function of energy by the MiniBooNE experiment.

In addition to this important issue there are other topics in this energy region likely to require work in the project X era. Some of those are: strange-spin of nucleon $(\Delta s)$ via NC elastic scattering and coherent production of pions and photons from nuclei.

\subsection{Higher-energy neutrino physics}

The "higher-energy" category of neutrino physics contains measurement made with $E_{\nu} \gtrsim 2.0 \mathrm{GeV}$ neutrino beams such as the current NuMI beam. Project $\mathrm{X}$ will allow further work in this regime by provided more POT in the 60, 120 proton energy regime.

The MINERvA experiment is currently working on this neutrino deep-inelastic scattering (DIS) physics with focus on relative and absolute cross section measurements, structure function extraction and the behavior of these quantities as the nucleus is changed.

In the Project $\mathrm{X}$ era, the challenge will be to improve the precision of these measurements. In order to do that, higher precision detectors will be required along with more intense better-known neutrino beams. One of the more important limitations of current measurements with the NuMI beam is the uncertainty on the absolute flux. To make the jump in precision desired for this DIS physics, a new type of beam, not utilizing pion DIF, is required. One solution for that is a neutrino factory where a well-known beam of muons decay yielding a intense well-understood neutrino flux. It may be that a big step in this direction can be possible with Project $\mathrm{X}$ and a muon DIF beam (such as the nuSTORM project).

\section{$2.4 \quad \nu_{\mu}-e$ elastic scattering measurements}

High statistics samples of neutrino-electron scattering events can be used to (1) search for the $\nu_{\mu}$ magnetic moment, (2) measure the weak mixing angle, $\sin ^{2} \theta_{\mathrm{w}}$ and (3) provide a calibration of the neutrino flux. These measurements may be done at a variety of energies accessible with Project $\mathrm{X}$. However, for the $\sin ^{2} \theta_{\mathrm{w}}$ measurement, which stands out as the most accessible and important physics topic, the prospect of an intense pion DAR beam at $E_{\nu} \approx 50 \mathrm{MeV}$ should be pursued and highest priority.

The present limit on the $\nu_{\mu}$ magnetic moment of $6.8 \times 10^{-10}$ Bohr magnetons is not nearly as good as the limit on the $\nu_{e}$ magnetic moment of $0.5 \times 10^{-10}$ Bohr magnetons. However, large samples of electron scattering events obtained with DAR or DIF neutrino beams could substantially 
improve the $\nu_{\mu}$ limit, perhaps to the $10^{-10}$ Bohr magneton level.

Neutrino-electron elastic scattering can provide valuable measurements of the weak mixing angle at various values of $Q^{2}$. A well-designed DAR neutrino beam can be used to measure the weak mixing angle at very low $Q^{2}\left(\approx 10 \mathrm{MeV}^{2}\right)$ with a sensitivity perhaps comparable to that of existing parity violation experiments $(0.5 \%)$. This would require a proton beam energy around $1 \mathrm{GeV}$ to avoid $\nu_{e}$ from kaon decays, high suppression of neutrinos from the $\pi^{-}, \mu^{-}$decay chain and a short proton beam spill, ideally $\approx 200$ ns. The $\nu_{\mu}$ from $\pi^{+}$decays appear promptly while the $\nu_{e}$ and $\bar{\nu}_{\mu}$ scattering events appear with the muon lifetime. The ratio of prompt to delayed electron scattering events determines $\sin ^{2} \theta_{\mathrm{w}}$.

The cross section for electron scattering is very well known and thus elastic scattering can provide an excellent determination of the normalization of the neutrino flux, limited only by the uncertainty of the measurement. The MINERvA experiment is working on using this scattering channel to constrain the NuMI on-axis flux. Current analysis indicates that $\approx 8 \%$ statistial error is possible for the NuMI low-energy configuration with improvement to $\approx 3 \%$ for the medium-energy.

\subsection{Dark sector searches}

Battel talk summary here.

\section{$3 \quad$ Future detector development}

In order to be build state-of-the-art detectors in the Project X timeframe, it will be necessary to do $R \& D$ on detector technologies so that experiments may be ready to deploy them. The following sections summarize the discussion on R\&D for scintillation and liquid Argon detectors.

\subsection{Scintillation detectors}

Liquid scintillator provides neutrino interactions via various detection channels and is an excellent detection medium for neutrinos with $E_{\nu} \approx 10 \mathrm{MeV}$ and also up to $E_{\nu} \approx 1 \mathrm{GeV}$ if the NC background is well-understood. A large, liquid scintillation detector is known for its multiple physics applications; such as solar, geo, reactor, and supernova neutrino detections short/long baseline neutrino oscillation; and could provide the golden-channel for proton decay via its kaon-capture before decay.

Organic liquid scintillators (LS) have a variety of selections from pseudocumene (PC) to the newly identified, linear alkylbenzene (LAB), which is environmentally safe with high flash point. The physics uses for LS are greatly expanded with the loading of metallic ions of interest; such as triple tags of In-loading for low-energy neutrino spectrum, Te- or Nd-loadings for double-beta decay, or Gd- or Li-loadings for neutron tagging (as antineutrino detection or veto solvent for solid-state or LAr detectors). The synthesis and purification techniques for organometallic-loaded LS (M-LS) have been largely developed (and the R\&D for different metallic ions of selections to be loaded in LS has been continuing for different physics of applications). The stability, optical transmission, and photon yield have been demonstrated to meet the critical challenges of experimental requirements by the successful Daya Bay and other reactor experiments.

Here we propose three liquid scintillation systems for future particle-physics experiments that may be executed at Project-X: (1) binary system of pure liquid scintillator mixed with inert solvents, such as mineral oil or dodecane; (2) singular system of pure LS (LAB preferably) with controls of fluor/shifter to potentially preserve the Cerenkov radiation; and (3) the newly developed waterbased liquid scintillator, a large, economic detector capable of scintillation and Cerenkov detection. 


\subsection{Liquid argon TPCs}

In the U.S., a precise technology choice for a state-of-the-art fine-grained, high-resolution neutrino detection method has been recently made: the Liquid Argon Time Projection Chamber (LArTPC). Current developments on LAr Technology are continuously progressing and actively pursued. These include: electron charge drift over long distance, cryostat insulation schemes and developments, cold read-out electronics vs. warm electronics for signal-to-noise optimization, event reconstruction and off-line code developments, ionization charge signal extraction in alternatives to wires, scintillation light signal extraction, and LAr response characterization with charged-particle beam tests.

The path toward the Intensity Frontier is now addressing the short-baseline anomalies at FNAL, with the MicroBooNE experiment - presently under construction - and with the LAr1 experiment, just proposed to the FNAL-PAC both based on LArTPC detectors.

The MicroBooNE experiment, with a LArTPC of $86 \mathrm{t}$ of active mass (170 t total) is expected to start data taking on the Booster Neutrino beam line at FNAL in 2014, many components are under fabrication (e.g. wire production already completed) and detector construction is being started at FNAL. The superior LAr technology will allow to address the MiniBooNE low-energy excess revealed in neutrino mode with a most efficient $e$-to- $\gamma$ separation capability.

In conclusion, the FNAL Program is very rich. A strong effort for the definitive assessment of the LarTPC technology is currently being pursued at FNAL, in particular with the beam test program at the Fermilab Beam Test Facility. The short-baseline program in the short/mid term at FNAL with MicroBooNE, for the definitive clarification of the neutrino-anomaly, and with LAr1 for the anti-neutrino anomaly, has great potential. All this will definitively contribute in opening the way toward the Intensity Frontier neutrino program with Project X.

\subsection{Single phase low energy threshold LAr detector}

An important aspect of $R \& D$ for any of these DAR neutrino measurements is the beam coincident neutron flux which is the most serious background of for these experiment. In order to properly understand the neutron fluxes at the potential experimental sites, an extensive simulation of neutron flux at $20 \mathrm{~m}$ away from the target has been carried out. We propose to carry out background estimations of the beam induced neutrons at BNB using 10-kg size of prototype liquid argon detector to measure neutron fluxes in the energy range of the region of interest. Developing a tonscale ultra-low energy threshold $(<20 \mathrm{keV})$ neutrino detector is another important $\mathrm{R} \& \mathrm{D}$ subject.

\section{Roadmap for Project X}

The sections summarized the potential neutrino physics discussed at the PXPS12 workshop under the purview of this working group. In order to build experiments to do this neutrino physics (along with that from the other working groups), a plan will need to be worked out that takes into consideration things such as:

- Timing with other experiments. Which of these physics measurements may be done by the time Project X begins.

- Optimum neutrino source design. How many different sources need to be built. Can one get DIR and DAR sources from same source? Can some of this physics be done with existing sources and more POT or with slight modifications?

- Proton economics. How are protons shared between the different programs and how does that change over time. 
- Duty factor. Many, if not all of these experiments will be better done with the low beamunrelated backgrounds associated with a low duty-factor $\left(\lesssim 1 \times 10^{-4}\right)$ beam. In that case, either the booster or a storage ring/linac combination would be used.

- Optimal detector design. The sections below lay out the some starting ideas for working through this.

\subsection{Conceptual Design}

As Project X proceeds through the various stages, the Booster and the Main Injector will still be running and therefore neutrinos may still be delivered from the sources on those machines. As the 1 and $3 \mathrm{GeV}$ linac of Project $\mathrm{X}$, new neutrino targets will be possible, perhaps sharing infrastructure with the targets for muon, neutron, etc production.

The final,staged conceptual design will need to work out a timeline for neutrino source from existing/modified/new production facilities for candidate experiments. This may be done in a clever, economical fashion, but it will take careful design.

\subsection{Detector R\&D}

The detector R\&D identified in the PXPS12 workshop required for Project X neutrino projects consisted of:

- New types of liquid scintillator such as LAB and water-based.

- Improvements in segmentation and readout to build large, economical, room-temperature scintillator detectors that can provide more fine-grained and complete information about neutrino interactions.

- Further work with liquid argon TPC detectors to better exploit both the ionization and scintillation and to more efficiently use the wealth of information provided by these detectors.

- Development of ton-scale single phase low-energy threshold liquid argon neutrino detector for coherent scattering measurement

- Various improvements in materials, readout, and analysis in conjunction with topics identified by other working groups with overlapping goals.

\subsection{Theoretical Work}

In order to support the experimental neutrino physics program and in order to make best use of the results, support of a theoretical program is required. This program will need to address some difficult issues that will require more work over next 5-10 years. In particular, the fairly intricate nuclear physics that arises when interpreting results from neutrino physics experiments that are performed on nuclear targets. 


\section{Advanced Water Cherenkov R\&D for WATCHMAN}

A.Bernstein'1, M.Bergevin², N. Bowden¹, S.Dazeley¹, S.Dye3, C.Grant², J.Learned 3 , J.Maricic ${ }^{3}$, P. Marleau, D.Reyna ${ }^{4}$, M. Sweany ${ }^{1}$, R.Svoboda 2 , M. Vagins' ${ }^{5}$ K. Van Bibber6, M. Wetstein ${ }^{7}$, M.Yeh ${ }^{8}$.

${ }^{1}$ Lawrence Livermore National Laboratory, ${ }^{2}$ University of California, Davis, ${ }^{3}$ University of Hawaii, Manoa,

${ }^{4}$ Sandia National Laboratory, ${ }^{5}$ University of California, Irvine, ${ }^{6}$ University of California, Berkeley, ${ }^{7}$ University of Chicago, ${ }^{8}$ Brookhaven National Laboratory

It has been shown by KamLAND that long-distance monitoring of an ensemble of reactors is possible using a one-kiloton Liquid Scintillator (LS) detector [1]. In addition, the development of very large (megaton scale) Water Cherenkov Detectors (WCD) is underway for future long-baseline neutrino experiments (e.g. Hyper-Kamiokande) $[2,3]$. Thus, given the success of small (few tons) neutrino detectors to passively obtain detailed information on the operation of commercial reactors [4], it has become a goal of the Nuclear Non-Proliferation (NNP) community to demonstrate the potential for water-based megaton-scale neutrino detectors for long-range monitoring. A collaboration of U.S. universities and laboratories (a.k.a. WATCHMAN) is now conducting a DOE-sponsored site search for a kiloton scale advanced water detector demonstration. The goal is to show that the backgrounds and efficiencies can be understood and controlled to a level sufficient to proceed to the realization of long-range monitoring. This presents an opportunity for groups developing technology for future water Cherenkov detectors to use this facility to develop and test new technology and methods. These studies could include:

Large Area Photosensors: The development of square meter scale MicroChannel Plates (MCP's) is an R\&D priority due to the physics potential offered from $\sim 60$ psec timing and few $\mathrm{mm}$ scale photon position resolution. The impact on vertex resolution and track fitting would be substantial, allowing for reconstruction of more complex neutrino interactions with multiple sub-vertices and tracks. This would result in reduced backgrounds from non-QECC events in long baseline experiments, and also less background in proton decay searches. At accelerators, these devices would provide superior discrimination for particle ID using ring-imaging detectors. At low energies, it might be possible to separate positron vertices from neutron capture vertices in reactor antineutrino-induced inverse beta decay events. The WATCHMAN detector provides a test tank of sufficient size and sensitivity to test the devices and their effectiveness in enhancing reconstruction.

Water-Based Liquid Scintillator: Brookhaven National Lab has recently developed a Water-based Liquid Scintillator (WbLS) that gives relatively fast scintillation light with useable light yields. If possible at a large scale, this would have a dramatic impact on the ability to look for SUSY-mediated proton decay, reactor neutrinos, geoneutrinos, and neutrinos from relic SN (getting rid of below-Cherenkov threshold neutrino-induced muons that make up much of the background). In addition, neutron tagging would improve background rejection for all possible proton decay modes via detection of final state neutrons. WATCHMAN would benefit greatly from this enhancement, as efficiency would rise by factors of 2-3 above that expected from pure water detectors. Any HEP detector now using organic liquid scintillator could now also consider the much cheaper and less toxic WbLS. WATCHMAN would provide the first large-scale deployment of such technology.

Gadolinium Doping: This would allow detection of neutron captures in water even without WbLS via Gd(n, $\gamma)$, which gives an $8 \mathrm{MeV}$ gamma cascade versus a $2.2 \mathrm{MeV}$ gamma from $\mathrm{H}(\mathrm{n}, \gamma)$. Neutron detection is crucial for reactor antineutrino detection (and thus critical for WATCHMAN) but would also allow better detection of relic supernovae and reduction of proton decay backgrounds (for reasons similar to $\mathrm{WbLS}$ ). While tests are being done in the 200 ton EGADS tank in Japan, the WATCHMAN detector would provide an order-of-magnitude scale-up of this technique from EGADS. WATCHMAN would thereby allow a substantial fiducial volume to be defined in the center of the detector, and consequently will have direct sensitivity to the reactor antineutrino signal of interest for remote monitoring.

Development of Enhanced Photomultipliers: R\&D has been done for LBNE to enhance classical PMT's with wavelength shifting films, peripheral plates, and/or reflectors. In addition, a 11-inch PMT is being developed by ADIT. WATCHMAN would allow deployment of these devices as a test prior to their use in Hyper-Kamiokande.

To summarize, participation of HEP groups in WATCHMAN for detector R\&D provides a substantial opportunity for leveraging of resources for future experiments. In addition, the technologies presented here would have substantial social benefits in terms of commercial applications and nuclear non-proliferation. 
References:

[1] "Precision Measurement of Neutrino Oscillation Parameters with KamLAND". The KamLAND Collaboration. Phys. Rev. Lett. 100, (2008) 221803

[2] "Letter of Intent: The Hyper-Kamiokande Experiment", arXiv:1109.3262, The Hyper-Kamiokande Working Group. [3] "LBNE Conceptual Design Report”, arXiv:

[4] Bowden, N.S., A. Bernstein, S. Dazeley, R. Svoboda, A. Misner and T. Palmer. Observation of the Isotopic Evolution of PWR Fuel Using an Antineutrino Detector. J. Appl. Phys. 105:064902 
Title: Liquid Argon Time Projection Chamber (LArTPC)

Time Frame: Short to Medium

Physics Justification: The Liquid Argon TPC is rapidly becoming the detector of choice for neutrino experiments because of its excellent tracking and calorimetric capabilities, and because of the high density and relatively low cost of Argon as a TPC tracking medium, leading to scalability to very large target masses. LArTPC development offers many technical challenges. Safety considerations are paramount given the cryogenic liquid involved, the use of very high voltages and high voltage feed-throughs, and the potential hazards of rapid conversion to a gas in the advent of loss of thermal or structural containment. The primary benefit of LArTPCs to neutrino experiments is their exceptional position resolution ability, facilitating selection of candidate interactions with very high signal purity and efficiency. LArTPCs can also be used for dark matter and proton decay experiments.

Technical Capabilities: LArTPCs have 3D space-point position resolution less than $1 \mathrm{~mm}$. They are also calorimeters and can detect energy depositions on the scale of $10 \mathrm{MeV}$ or less. Low energy thresholds make them ideal detectors for a wide range of applications for neutrino experiments, from solar and supernova neutrinos to accelerator beam and atmospheric neutrinos. These detectors are also useful for dark matter experiments and can be used to tag dark matter interactions through a combination of ionization charge and scintillation light information. LArTPCs placed in underground locations can also be used to search for proton decay given their high efficiency for observing decay modes such as a proton decaying to a kaon and neutrino.

Technical Requirements: A primary requirement for LArTPCs is the elimination of electronegative contaminants from the LAr to the level of approximately 100 parts per trillion of oxygen equivalent contamination. Other requirements include the ability to provide very high voltage to the TPC in order to create drift fields on the scale of $500 \mathrm{~V} / \mathrm{cm}$ over several meters and the development of low-noise front end electronics that can operate reliably within the liquid argon volume over tens of years. The detection of the scintillation light is also very important for neutrino, dark matter and proton decay experiments requiring the use of wavelength shifters and efficient large-area photodetectors. The detection of scintillation light requires low contamination of nitrogen in the liquid argon as well. Dark matter experiments will also need low radioactivity liquid argon to suppress noise at $\mathrm{keV}$ energy levels. Proton decay experiments will need to be able to observe scintillation light to discriminate between proton decay events and cosmic ray induced activity near the edges of the volume.

Industrial Involvement: Industry has improved its ability to provide liquid argon with low (ppm) levels of water, oxygen and nitrogen contamination over the last decade. Developments in the Liquefied Natural Gas storage and transportation industry have led to cost-effective vessel construction techniques.

\section{Key Motivations for this Detector R\&D:}

$\checkmark$ Increases physics capabilities for neutrino, dark matter, and proton decay experiments. 


\title{
Liquid Scintillator Instrumentation for Physics Frontiers
}

\author{
Minfang Yeh \\ Brookhaven National Laboratory, Upton, NY 11973, U.S.A
}

The technical challenges of liquid scintillator instrumentation for future physics frontier research are summarized in this paper. Liquid scintillator has played a key role in the history of neutrino physics since the first direct detection of antineutrinos by Reines and Cowan in 1956. Key parameters for research and development are stability, attenuation length, photon yield, and intrinsic radioactivity. With the recently revealed, large value of the $\theta_{13}$ mixing parameter, various topics ranging from determination of neutrino mass hierarchy via oscillation or neutrinoless double beta-decay to studies of proton decay, astrophysical and supernova neutrinos have been proposed for potential deployment in the next decades. The various experiments pose different instrumentation challenges that some are common features between different detectors while others require specific research and development for individual detector. In general, new scintillation liquids with high flash point and less reactivity (i.e. linear alkylbenzene, LAB) or cost-effective with nontoxic liquid handling (i.e. water-based liquid scintillator, WbLS) are needed for a variety of future scintillator-based experiments. Conversely the stability of metaldoped scintillator has always been a central area of research and development since the abortive deployments by early experiments (i.e. Chooz, Bugey-3). Although advanced organometallic loading technologies by either liquid-liquid extraction or direct dissolution of organometallic compounds have been developed for the recent reactor experiments (e.g. the stability of gadolinium-loaded scintillator is now extended over several years to the experimental lifetime), the stability of scintillator doped with certain metallic ions, particularly lithium, boron, and some double beta decay isotopes, has not yet been proven. The detector requirements and necessary research and development for a variety of these experiments are described below.

\section{Long Baseline Reactor Neutrino: A super bright and transparent liquid scintillator.}

The hierarchy of neutrino mass states is as of yet unknown and may hold the key to understand the nature of neutrinos and their masses in the new Standard Model. While measurement of mass hierarchy through reactor antineutrino disappearance is independent of the $\mathrm{CP}$ violating phase and matter effects, a precision oscillation experiment over $\sim 60 \mathrm{~km}$ baselines requires superior energy resolution of $<3 \% / \sqrt{E}$ and sub-percent level understanding of the energy response to distinguish the normal and inverted hierarchy oscillation patterns. Such an experiment would also make precision measurements of $\theta_{12}, \Delta \mathrm{m}_{21}^{2}$ and $\Delta \mathrm{m}_{32}^{2}$ and potentially be sensitive to additional physics such as geo-neutrinos, solar 
neutrinos, atmospheric neutrinos and proton decay. The technical challenges of achieving this energy resolution and a nonlinearity measured to a fraction of $1 \%$ over the detector volume require extensive scintillator research and development. The crucial factors are (1) high intrinsic light-yield of $\sim 15,000$ optical photons per $\mathrm{MeV}$ and (2) superior optical attenuation length of $30 \mathrm{~m}$ or better. The latter is particularly critical for a multi-kton-scale detector. Up to now, none of the currently deployed scintillators can achieve these requirements. Searching for a non-hazardous, brighter scintillator, utilizing extensive purification, and optimizing the combination of fluor and wavelength shifters that could shift the scintillation emission to a highly transparent region (i.e. $\sim 450 \mathrm{~nm}$ ) are the keys to this scintillator development. For detector nonlinear energy response, establishing a loading method to dope short halflife particle sources $\left(\mathrm{e}^{+}\right.$or $\left.\mathrm{e}^{-}\right)$with kinetic energies of $2-6 \mathrm{MeV}$ in scintillator for calibration deployment will provide a direct measurement of energy response.

Short baseline reactor neutrino: A chemically stable Li- or Gd-doped scintillator with improved background rejection.

Anomalous results from a variety of neutrino experiments indicate either a sign of new physics or unknown physics in the reactor flux predictions. It has been suggested that such a deficit between measured and predicted reactor $\bar{v}_{e}$ fluxes may be the signature of additional sterile neutrino states with mass splittings of the order of $\sim 1 \mathrm{eV}^{2}$ and oscillation length of $\partial(3 \mathrm{~m})$. A measurement of both the $\bar{v}_{e}$ rate and energy spectrum as a function of distance from the reactor core at short baselines can be used to perform a definitive search for such oscillations. Since reactor-related neutrons and cosmic muons in a surface detector cannot be easily shielded, background suppression will be a major technical challenge in the design of such a detector. Although segmentation, combined with an active muon veto, has been proven to reduce muon background effectively, the primary approach to this problem will be reliable event identification, either through the use of a delayed neutron capture signal combined with pulse shape discrimination (PSD) or event track reconstruction. By doping the scintillator with a high neutron capture cross-section isotope and requiring a coincidence between the initial IBD positron signal and subsequent neutron capture signal, background neutrons can be efficiently rejected. Gadolinium-doped scintillator has been proven in reactor $\bar{v}_{e}$ experiments. However, the high energy gammas in the final state create difficulties for accurate position reconstruction in small to medium sized detectors. On the other hand, lithium-doped scintillator through the reaction of ${ }^{6} \mathrm{Li}+\mathrm{n}_{\mathrm{th}} \rightarrow \mathrm{t}+\alpha(4.8 \mathrm{MeV})$, being highly localized in the detector, can lead to precise position reconstruction and improved background rejection. However the stability of lithium-doped scintillator stands as a challenge due to its hydrophilic nature (i.e. Li-LS from Bugey-3 deteriorated after a few months of deployment). Recently a new organometallic loading model based on the water-based principal (WbLS) offers attractive alternatives with different characteristics and 
will be used as the primary approach to investigate the enhancement of Li-LS stability. The choice and composition of either Gd- or Li-doped scintillators will be driven by light-yield, timing of the delayed coincidence signal, accidental background suppression, energy response and possible veto efficiency against muons.

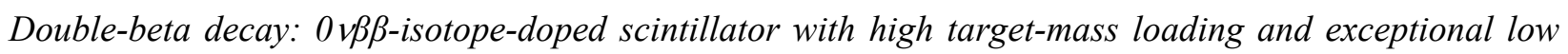
intrinsic radioactivity.

Much of neutrino mixing mechanism has been learned over the past decades with few remaining unknowns of absolute magnitude of neutrino mass and Majorana or Dirac nature of neutrino particle. Experimentally, a measurement of the rare radioactive decay process known as double beta decay (DBD) is the most realistic way to explore these neutrino properties. An observation of neutrinoless double beta decay $(0 \vee \beta \beta)$ would conclude that the massive neutrino particles are Majorana and could provide an opportunity of measuring absolute neutrino mass magnitude or setting its scale at the Majorana effective limit. Attempts to detect $0 v \beta \beta$ signal have crossed over several decades with various detection schemes separated by their abilities of distinguishing electron-sum energy spectrum that are dominated by radioactive background and resolution. Besides statistics precision, the key to a successful $0 v \beta \beta$ detection is then the enhancement of signal ratio by suppressing the inherent or external backgrounds that could exceed the $0 v \beta \beta$ Q-value.

Among a variety of double-beta-decay experiments, $\mathrm{SNO}+$ and KamLAND are the present scintillator-based detectors that use candidate $0 v \beta \beta$ isotope to serve as detector and target. Such $0 v \beta \beta$ isotope doped scintillators have the advantages of scalability and flexibility that the target mass of the chosen isotope can be expanded to larger scales without significant increases of external background or construction cost if the inherent purification is applied to the core materials. SNO+ originally proposed ${ }^{150} \mathrm{Nd}$ (neodymium; $5.6 \%$ in nature) as the $0 v \beta \beta$ target due to its high 4-MeV Q-value; however the mass of $\mathrm{Nd}$ doped in scintillator is statistically limited due to its nature of absorption that an optimization is set to be $0.3 \%$. Yet other $0 v \beta \beta$ isotopes (i.e. calcium, tellurium, selenium, molybdenum, and germanium) are attractive; their hydrophilic natures indicate that loading of these isotopes in scintillator by conventional complexing-ligand, $-\mathrm{COOH}$ or $\mathrm{P}=\mathrm{O}$, method will be difficult. Similar to the approach of short baseline scintillator detector, the successful water-based loading model could enable the loading of these wateraffinity isotopes in any scintillator of interest, which opens a new avenue for future particle physics experiments of metal-doped scintillation detector. SNO+ has now officially selected tellurium (Te) as the new $0 v \beta \beta$ target due to the high abundance of ${ }^{130} \mathrm{Te}(33.8 \%, \mathrm{Q}=2529-\mathrm{keV})$ and a factor of $\sim 30$ times less of $2 v$ background than that of ${ }^{150} \mathrm{Nd}$. Moreover the scalability to a higher loading of Te in the scintillator 
for a ton-scale $0 v \beta \beta$ experiment becomes possible in the near future. The current baseline of a $0.3 \% \mathrm{Te}-$ doped, LAB-based scintillator has been successfully developed (comparable $\mathrm{m}_{\beta \beta}$ sensitivity to that of CUORE). Assessments of performance and stability for such Te-doped LS are ongoing and extensive purification methods to remove any inherent and cosmogenic-induced radioisotopes from the core materials are under development. Research and development aiming to increase the target-mass at the percent level $(1-3 \%)$ to probe $m_{\beta \beta}$ sensitivity reachable at the hierarchy region is the goal.

\section{Proton decay: Scintillation water for physics below Cerenkov}

The approach of a large water-based liquid scintillator (WbLS) is to develop a new detection medium, especially, in search for proton decay. The $p \rightarrow K^{+} \bar{v}$ mode in large water Cerenkov detectors is invisible by the kinematics of the initial $K^{+}(105 \mathrm{MeV}$, below the Cerenkov threshold). The current best limit of $\tau\left(p \rightarrow K^{+} \bar{v}\right)>2.8 \times 10^{33} \mathrm{y}$ at $90 \%$ C.L. comes from Super-Kamiokande, which uses a 6.3-MeV $\gamma$ ray transition to tag proton decays inside oxygen nuclei. The addition of scintillator to a water Cerenkov detector will have two consequences: ionizing particles below the Cerenkov threshold in water $(\beta<0.75)$ become detectable by their scintillation light, and the atmospheric $\nu_{\mu}$-induced $\mu^{+}$background can be further suppressed. A full Geant4 Monte Carlo simulation of a currently developed scintillation water at 90 optical photons per $\mathrm{MeV}$ and $\lambda_{1 / \mathrm{e}} \sim 60 \mathrm{~m}$ in a SK-like cylindrical detector shows that after 10 years of run time, only 0.1 background events are expected and a limit of the proton lifetime at $\tau>2 \times 10^{34} \mathrm{y}$ at 90\% C.L., an order of magnitude improvement over the current limit, can be achieved. In addition, the ability to detect the 2.2-MeV capture gamma will reduce background for essentially all modes, as real proton decay events are unlikely to be accompanied by a free neutron, whereas atmospheric neutrino events at proton decay energies will often have a neutron in the final state. Furthermore the WbLS is capable of loading any metallic ions of interest (i.e. Gd-, ${ }^{6} \mathrm{Li}$, or ${ }^{10} \mathrm{~B}$ for neutron tagging enhancements). The same water-based scintillator could also serve as a near detector for T2K and Hyper-K or be used for detection of diffuse neutrino flux from distant past supernovae.

At large, the water-based liquid scintillator is a cost-effective detector option for future massive detectors with a unique capability of exploring physics below Cerenkov. The principal of a massproducible $\mathrm{WbLS}$ has been demonstrated at lab-scale with stability over $2+$ years since preparation. The ability of capturing particles below Cerenkov threshold by scintillation light was proven by low-intensity proton-beam in the energy region of $200-2000 \mathrm{MeV}$ (above and below Č threshold) at BNL-NSRL facility. A deployment of such a scintillating water detector at deep underground laboratory with careful controls of low-energy radioactive background could reach the sensitivity of the SUSY GUT prediction of 
$10^{34 \pm 1} \mathrm{y}$ with other implications in neutrino and rare-event physics; an economic detector with extensive physics potentials for the next-generation physics-frontier experiments.

Dark Matter: An ultra-clean scintillator with neutron-capture-enhanced isotopes.

The most popular candidate for dark matter is the WIMP (Weakly Interacting Massive Particle). Experimentally this could be detected through its collision with atomic nuclei and subsequent nuclear recoil with a velocity that is thousands of times that of sound. For such measurements, the surrounding neutron background plays a critical role since it also causes recoils of atomic nuclei that are indistinguishable from the expected WIMP signal. In addition, neutrons induce gamma-rays through (n, $\gamma$ ) and $\left(\mathrm{n}, \mathrm{n}^{\prime} \gamma\right)$ processes (in the detector or shielding) and may produce long-lived radioactive isotopes. A comprehensive identification and rejection of such backgrounds are absolutely crucial to reliably discover a WIMP signal. Consequently a neutron detector adjacent to a dark matter detector for direct assessment of such backgrounds is essential. This neutron detector can be filled with a liquid scintillator doped with a neutron-capture-enhanced isotope. In addition to stability and light yield, the main research and development for such a neutron-tagging scintillator is high detection efficiency and ultra-low radioactive backgrounds $(\sim 0.1 \mathrm{~Hz})$ from inherent radioisotopes of $\mathrm{U}$, Th, Ra, and Rn. Extensive purification and the ability to probe the radioactivity of doped scintillator below the level of part per trillion in a low-intensity counting facility have to be established. Purification methods with separation column, exchange resin, pre-concentration and solvent extraction, followed by NAA, ICP-MS, and other detection means, need to be developed.

\section{Other Physics: Neutron-tagging, Nonproliferation, and Medical Imaging Applications}

The development of innocuous scintillation liquids (LAB- or water-based scintillator) not only enhances the applications of such a detector to be used at highly security-tightened areas (reactors) or in confined space (underground), also simplifies the preparation and handling of large chemical solvents. Inexpensive large neutron detectors could be applied for nonproliferation through the monitoring of containers to detect transport of radioactive materials. They could also be beneficial as an economic active veto shield against cosmic-induced muons or in medical imaging with segmented tracking arrays. 
2 Cosmic Frontier

clxxviii 


\title{
A readout architecture for 100,000 pixel Microwave Kinetic Inductance Detector
} array.

\author{
Gustavo Cancelo (Fermilab), Juan Estrada (Fermilab)
}

This paper defines a compact, self-contained readout architecture for $100 \mathrm{~K}$ pixel MKID readout by the end of FY2016. A readout system for $100 \mathrm{~K}$ pixles is challenging in several fronts:

- Low system noise covering a frequency spectrum that goes from DC to microwave RF.

- Highly multiplexed RF system.

- Mixed analog and digital electronics.

- High data throughput.

- On board digital signal processing.

- Temporal and spatial synchronization.

- Complex system calibration.

- Data processing pipeline.

System architecture:

The most advanced MKID readout camera today is ARCONS [1] that has $2 \mathrm{~K}$ pixels and has been successfully tested at Monte Palomar in 2012 (i.e. Palomar 200" and Lick 120" telescopes for 24 nights of observing). The readout system used for ARCONS is the ROACH that can read out up to 256 resonators per feed line in $512 \mathrm{MHz}$ of bandwidth, with $2 \mathrm{MHz}$ spacing between tone frequencies. As MKID cameras get larger a larger readout system is required. In this paper we describe an architecture to readout up to $100 \mathrm{~K}$ pixels. A $100 \mathrm{~K}$ pixel camera requires a focal plane with a mosaic that has yet to be defined. There are several technical issues that constrain the optimum number of pixels per RF feed line, pixel to pixel frequency separation on a single feed line, etc. Henceforth, some common sense assumptions are made for this paper.

The channel separation on the same feed line is $2 \mathrm{MHz}$ for adjacent channels. A $1 \mathrm{MHz}$ may be possible in the future which allows to duplicate the number of channels per RF line or to reduce the complexity of the RF electronics.

A maximum number 2000 channels per RF feed line. That imposes a requirement of $4 \mathrm{GHz}$ per feed line. A total of 50 feed lines to complete $100 \mathrm{~K}$ channels. 


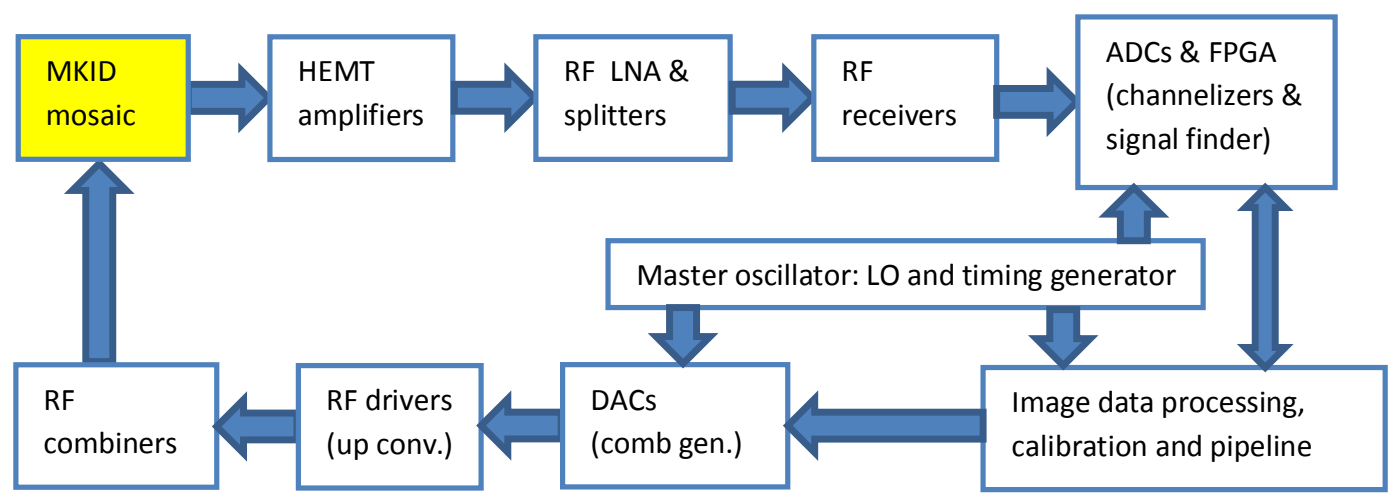

Figure 1: 100K pixel readout architecture

\section{RF input output}

Ideally a single RF line receives input from a DAC and delivers output to an ADC but in order to optimize bandwidths and sampling frequencies a slightly different input/output mapping may be required. The input and out mapping is done with RF power combiners and splitters. Power combiners and splitters add some noise and insertion loss.

MKID signal detection and noise MKIDs work on the principle that incident photons change the surface impedance of a superconductor through the kinetic inductance effect [2]. MKIDs have a large optical dynamic range and the number of quasiparticles generated is a function of the number and energy of photons that deposit energy on that inductance. The inductance is matched to a capacitance to form a microwave resonant circuit coupled to an RF feed line. The signal is detected by observing the amplitude and phase change of the current (or voltage) in the feed line. The responsivity of the pixel is a function of electrical and mechanical parameters and is adjusted to cover the desired dynamic range.

One of the most attractive characteristics of MKIDs is single photon energy discrimination. The energy resolution is a function of the energy gap of the material operating in superconducting mode. Although the current optical MKID technology achieves a spectral resolution of 10 at $400 \mathrm{~nm}$ it is expected that the resolution will approach or even exceed 100 in the near future.

The current proposal assumes a resolution of 100, which is sufficient for mid resolution spectroscopy for dark energy, and calculates the electronic noise requirements. The resolution is limited by the MKID noise or by the first amplifying stage, currently a HEMT amplifier.

An MKID pixel operates at a typical power between -85 to $-100 \mathrm{dBm}$. The HEMT noise temperature is about 5 to 6 kelvin for the 2 to $8 \mathrm{GHz}$ spectrum. Such a noise temperature gives a total noise power of $1.4 \times 10^{-16}$ watts over a $2 \mathrm{MHz}$ bandwidth. The warm electronics should be able to excite and readout 2000 pixels in an RF feed line without introducing extra noise to the MKID-HEMT system. Since the typical gain of a two stage HEMT is 35 to $40 \mathrm{~dB}$, the warm electronic noise should be below $-128 \mathrm{dBm}$. This number requires another stage of low noise amplification before the analog down conversion and digital sampling. 
The Electronic System Engineering Department at Fermilab has started the design of an electronic system based on low noise amplification RF up and down conversion and high speed sampling. The goal is to readout up to 2000 channels per feed line using 3.6 Ms/s 12 bit ADCs. The system noise temperature per channel referred to the input of the HEMT must be below the HEMT noise temperature. A block diagram of the system is depicted in Figure 1.

Highly multiplexed RF system

A large MKID camera requires a highly multiplexed RF system in order to minimize the number of feed lines into the cryostat. Every RF feed line increases the cooling power requirement, requires more HEMTs in the $4 \mathrm{~K}$ phase and more channels of warm electronics. The maximum number of channels on a feed line is limited by RF allowable bandwidths in the up and down converters and filters, phase noise, local oscillator noise (LO noise), ADC sampling frequency and ADC noise. The current goal is 2000 channels per RF feed line using $4 \mathrm{GHz}$ of bandwidth centered on the MKID central carrier frequency.

The warm electronics requires low noise microwave RF analog and fast sampling ADC and DAC conversion. Furthermore a highly multiplexed and compact system requires a compact design. Special care must be taken to minimize noise at all levels at the same time we maximize the number of channels per board and minimize the total number of boards. The RF electronics is sensitive to signal degradation and crosstalk at interfaces and connectors, LO leakage, and high frequency noise from the digital electronics. The ADCs are limited by quantization noise and channelization. Signal to noise ratios are low due to the extremely low power allowed by the MKID pixels. On resonance, a typical $S_{21}$ transmitted power is a few percent of $-85 \mathrm{dBm}$ or less. A typical ADC input is $10 \mathrm{dBm}$. Unless we place a large amplifying chain the ADC input is poorly utilized and dominated by quantization noise.

The analog down converter phase noise is typically dominated by the phase noise in the master oscillator (MO) as shown in Fig. 2 [3]. The $\mathrm{MO}$ noise couples through the local oscillator generating sidebands in the IF spectrum.

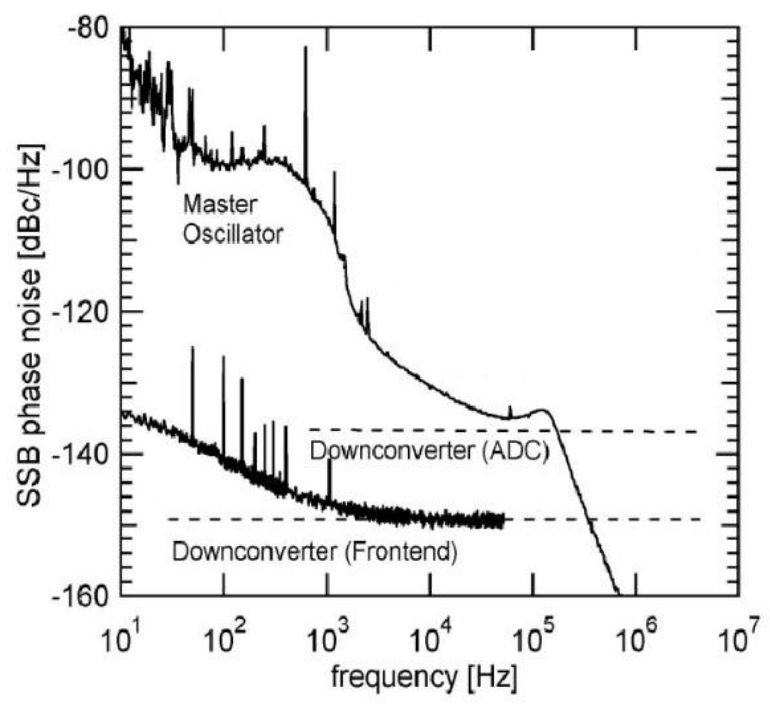

Figure 2 
Data throughput

At 3.6 Gs/s, $1.5 \mathrm{~B} / \mathrm{sample}$ each feed line generates $5.4 \mathrm{~GB} / \mathrm{s}$ and a total of $\mathrm{A} 270 \mathrm{~GB} / \mathrm{s}$ for a system of 50 feed lines. The output data bandwidth is considerably smaller. Each image is 100 Kpixles at 2 to $3 \mathrm{~B} / \mathrm{pixel}$ and a rate of 100 images/sec totals $30 \mathrm{MB} / \mathrm{s}$. That calculation implies that there is considerable signal processing with a data reduction of 1000 in the data acquisition system. Part of that data reduction is done by the channelizer and digital filter taking advantage of the large oversampling rate in the ADC. The channelizing and filtering is typically done by an FPGA because the algorithms are based of FFT and polyfilter bank techniques that require a high degree of parallelism. The output of the channelizer is 2 to $4 \mathrm{Ms} / \mathrm{s}$ to cover a 1 to $2 \mathrm{MHz}$ bandwidth/channel. The next processing step must fit the data to obtain pixel signal information. The fit of the complex signal $\mathrm{S}_{21}$ requires calibration, rotation and scaling and is done by an FPGA, a GPU or a general processor. The estimated pixel data is stored in memory for image assembly and pipeline processing.

Temporal and spatial synchronization

The system synchronization is provided by the master oscillator which is phase locked. The MO can be locked to a GPS which provides a 1pps synchronization signal. The DACs and channelizers must start at same time to provide a consistent phase for all the carrier bins. The absolute time of the day will be provided by the unix time.

DAC, ADC and FPGA synchronization is achieved using a phase locked loop clock distribution device with less than 200 femto second jitter. Data packets will contain a time stamp and a header indicating the spatial pixel location and the complex data.

System calibration

As part of the data taking procedure each pixel's raw phase response must be mapped to photon energy. For instance the ARCONS camera has developed a system to uniformly illuminate the focal plane with three lasers at the same time before and after science observations, thereby providing a reference to calibrate each detector's response.

The response for a single detector is given by a histogram using the number of counts for typically a minute of integration. The data is fit and processed to find the location of the laser wavelength providing the conversion to photon energy.

Data processing pipeline The data pipeline will be based on the ARCONS pipeline. Currently the following blocks have been developed or are under development:

- Barycenter Photon Arrival Times

- Cosmic Ray Cleaning

- Wavelength Calibration

- Flat field Calibration

- Spectral Shape Calibration

- Exposure Time Masking

- RA/Dec Determination

- Tip/Tilt Correction 
Timetable:

FY 2014:

Low noise amplifier (HEMT) characterization.

Implementation of $2000 \mathrm{ch} / \mathrm{RF}$ line using ultra-fast DACs and ADCs.

Design of a superconducting cable for 50 RF feed lines.

MKID system testing and characterization.

Firmware and software development.

FY 2015:

Multi transistor HEMT packaging.

High BW data signal processing.

Design of final RF up/down converters.

FY 2016:

Build the 100Kpixel readout system

Finalize firmware and software development.

Commissioning

[1]. "ARCONS: A 2024 Pixel Optical through Near-IR Cryogenic Imaging Spectrophotometer" B.A. Mazin, S.R. Meeker, M.J. Strader, B. Bumble, K. O'Brien, P. Szypryt, D. Marsden, J.C. van Eyken, G.E. Duggan, G. Ulbricht, C. Stoughton, M. Johnson, arXiv:1306.4674

[2] Mattis, D. C., \& Bardeen, J. 1958, Phys. Rev., 111, 412

[3] "Phase stability of the next generation RF field control for VUV and X-ray free electron laser", F.Ludwig, M.Hoffmann, H.Schlarb, S.Simrock, Proceedings of EPAC 2006, Edinburgh, Scotland, TUPCH188 


\title{
Instrumentation for New Measurements of the Cosmic Microwave Background polarization
}

\author{
Clarence L. Chang, John E. Carlstrom, Stephan S. Meyer
}

\section{Introduction}

The CMB has played a pivotal role in cosmology and particle physics. Its discovery in 1965 validated the hot Big Bang theory. Its homogeneity motivates the theory of Inflation. Its anisotropy shows that the geometry of our universe is flat and gives a complete census of the matter-energy content of the universe. $\mathrm{CMB}$ measurements remain critical to answering some of the most interesting questions confronting fundamental physics today. These questions at the heart of the HEP program mission: What is the Universe made of? Did the Universe start with an epoch of inflation? What is the energy scale of inflation? What is the fate of the Universe? What is dark energy? What are the neutrino masses? The importance of these questions is echoed in reports by the Committee on Elementary Particle Physics [? ] and the PASAG [? ].

CMB measurement is a sensitivity limited field of research. Since CMB detectors are background limited, increasing the sensitivity of CMB instruments is possible only through increasing the number of optical modes measured. Thus, the focus of research and development for CMB instrumentation is on scalable technologies. The challenges of increased production while controlling costs defines a critical role for High Energy Physics resources in sustaining US leadership in CMB science.

Current and upcoming CMB experiments are classified into stages. Stage II experiments measure $O(1000)$ optical modes and have recently or are about to deploy. Stage III experiments will deploy in the latter half of the decade and measure $O(10,000)$ modes. Stage IV experiments will observe $O(100,000)$ modes and are anticipated to deploy $\sim 2020$. This paper focuses on the technical requirements needed for Stage III and Stage IV experiments.

\section{Sensors}

The development of new detector arrays is the highest priority. Transition Edge Sensor (TES) Bolometers which use a superconducting film voltage-biased at the transition are currently the leading detector technology, in use in the large majority of CMB experiments including Stage II and proposed Stage III experiments. TES-based focal planes for Stage III and beyond will require development of new detector architectures and fabrication processes to enable mass production of large format arrays.

Microwave Kinetic Inductance Detectors (MKIDs) are another direct (phase-insensitive) detector technology, based on the change in inductance in a superconductor in the presence of photon-induced quasiparticles. MKIDs are read out using RF multiplexing which offers advantages of simplicity and large multiplexing factors. MKIDs do not yet demonstrate the sensitivity needed for CMB polarimetry.

High electron mobility transistor (HEMT) amplifiers have been used for coherent (phase-sensitive) detection. HEMTs are being combined with monolithic microwave integrated circuits (MMICs) to develop polarization modules that amplify and detect all the available polarization Stokes parameters. There is no clear path, however, for scaling HEMT-based focal planes to Stage III and IV sensitivities.

\section{Readout Electronics}

Stage III and Stage IV CMB experiments will require continued development of readout electronics in order to record timestreams for arrays of 10,000 to 100,000 detectors with low power deposition per element operating at $100 \mathrm{mK}$. 
TES bolometers may be read out with multiplexed readouts, either based on time-domain or frequencydomain multiplexing with SQUID (Superconducting QUantum Interference Device) current amplifiers. These SQUID-based multiplexer systems currently achieve MUX factors of $\sim 10$ detectors per readout channel and are currently in use with arrays of 10,000 detectors. Stage III and IV experiments will require MUX factors between 50-100. The development of RF multiplexed readouts is a potentially ground breaking technology enabling MUX factors of $\sim 1000$.

\section{Detector Coupling}

Next generation CMB experiments need high efficiency coupling from free-space radiation into the detectors with high polarization preserving fidelity. The focal plane designs require polarization detection, beam collimation, and band-pass filtering. Current (Stage II) approaches include antenna-coupled bolometers combined with feedhorns, lenses, or planar antennas. A similar integrated approach is being developed for HEMT-MMIC amplifiers combined with feed horns. These coupling schemes offer compact and modular designs that are scalable to mass production.

For Stage III and beyond, new developments in multi-chroic bolmetric detector arrays provide the promise of factors of a few increase in mapping speed per unit focal plane area. An alternative to large arrays of single-moded bolometers are multi-moded bolometric detector arrays, especially at the higher frequency end of the CMB. For a given optical aperture, these multi-moded architectures simplify the detector readout at the expensive of angular resolution. In addition, bare detector arrays are also being developed for CMB polarization experiments having increased reliance on optical components for beam control and polarization analysis.

\section{Increasing System Throughput}

Experiment mapping speed is proportional to the total optical throughput. High efficiency, largeaperture, large-field telescope design is needed. Some designs require the active polarization modulation systems with high throughput. Current work for Stage III experiments focuses on developing high-efficiency cryogenic components that will enable large focal planes that completely utilize the available optical footprint. Stage IV experiments will require development of techniques for mass production of these optical components.

\section{Summary}


Physics Driver: Future Atmospheric and Water Cherenkov $\gamma$-ray Detectors

Time Frame: Short to Long (2015-2030)

Physics Justification: Next-generation $\gamma$-ray experiments share common physics goals, including indirect searches for dark matter (DM) through annihilation signatures, searches for new physics (e.g. axion-like particles, or ALPs) imprinted on the spectra of distant astrophysical sources and tests of Lorentz invariance with astrophysical sources such as pulsars, active galactic nuclei, and $\gamma$-ray bursts. Searches for DM annihilation photons with $\gamma$-ray telescopes provide an essential complement to accelerator searches and direct detection methods. Indirect searches have the unique ability to confirm a DM candidate as astrophysical DM and permit us to set limits on portions of the DM candidate parameter space inaccessible to direct methods.

These science drivers impose a common set of requirements on future instruments. The reach of DM annihilation searches is driven by a $\gamma$-ray detector's sensitivity to faint emission, while the range of photon energies covered by the instrument translates directly into the range of potential DM particle masses. Searches for ALP or Lorentz invariance signatures benefit from high photon statistics and precision measurement of spectra or light curves. This consistently points towards a need for an order of magnitude improvement in sensitivity, $\sim \mathrm{km}^{2}$ effective area, and extended energy coverage $(\sim 30 \mathrm{GeV}-300$ $\mathrm{TeV}$ ). All of these science drivers also benefit from the increased survey capability enabled by a wide field of view (FOV). A large FOV and fine angular resolution are particularly valuable to searches for DM annihilation photons from the Milky Way halo or the Galactic Center, since they permit a DM signal to be more effectively disentangled from diffuse astrophysical backgrounds.

Future air and water Cherenkov instruments such as the Cherenkov Telescope Array (CTA) and the High Altitude Water Cherenkov observatory (HAWC) have complementary strengths and limitations.

HAWC's $>95 \%$ duty cycle and FOV covering $>15 \%$ of the sky make it a natural survey instrument, but it does not achieve fine energy and angular resolution $(100 \mathrm{GeV}-10 \mathrm{TeV}$ coverage; energy resolution $<50 \%$ above $10 \mathrm{TeV}$ only; angular resolution $\sim 0.1^{\circ}$ above $10 \mathrm{TeV}$ only). Future air Cherenkov instruments will push down the energy threshold to $\sim 30 \mathrm{GeV}$ and achieve excellent angular $\left(0.02^{\circ}-0.065^{\circ}\right)$ and energy resolution $(\sim 10-15 \%)$, but possess a narrower FOV $\left(\sim 8^{\circ}\right)$ and more limited duty cycle.

Technical limitations: Photosensor cost will be a major issue for both future air and water Cherenkov instruments, as will the problem of controlling the relative timing between distributed detectors to better than $1 \mathrm{~ns}$. For the large air Cherenkov telescope array approach, the increased camera size and weight with increased FOV, the cost of optical structures, and significantly increased data rates that require more effective triggering at either the camera and/or array level will also be a concern.

\section{Technical Capabilities:}

1) Study of new high quantum efficiency and large-area photodetectors

2) DAQ: Continued development of ASIC technologies to provide low-cost and low-power digitizers

3) Development of novel optical structures

4) New timing distribution schemes and trigger technologies based on ATCA and uTCA

Key R\&D Directions: Suggested key areas of R\&D with broad impact are:

$\checkmark$ Low-cost low-power digitizers

$\checkmark$ Low-cost high-performance photosensors

$\checkmark$ Methods of timing distribution across large scale arrays

$\checkmark$ Trigger electronics

$\checkmark$ Revolutionary telescope optics that impact camera weight and permit use of novel photosensors 


\subsection{Dark Energy}

\section{(Author: Gaston Gutierrez, Fermilab)}

\subsubsection{Physics motivations}

The current acceleration of the Universe is perhaps one of the biggest mysteries in science today. At a redshift of $\mathrm{z} \sim 0.8$ the Universe transitions from an expansion that is slowing down to one that is accelerating. A possible explanation for this acceleration is the presence of a vacuum energy throughout the Universe, known as Dark Energy (DE), which becomes dominant as the matter density decreases due to the expansion of the Universe. Since the acceleration is a late phenomena in the history of the Universe it mostly manifests itself through its effects on the evolution of the matter density after galaxies were formed. Two kinds of probes are used to measure the properties of DE: a) Distance measurements which allow us to track the expansion of the Universe, and b) The growth of cosmic structure, which will help determine if the acceleration is due to $\mathrm{DE}$ or to the break down of General Relativity (GR). Both these measurements require the precise determination of angles, distances and shapes (for galaxies) of stellar objects like galaxies, supernovae, gas clouds, QSO, etc. Assuming that all systematic error could be brought under control then the error in the parameters characterizing DE and departures from GR will improve as the volume of the measured Universe increases.

\subsubsection{Existing Technical Capabilities and Challenges}

Due to experiments currently running (DES, Panstars, ...) and being built and/or planned (MS-DESI, EUCLID, HETDEX, ...) the next decade will witness a large improvement in the amount of sky covered by telescopes. Due to these instruments the expected situation a decade from now can be summarized as follows. Imaging: about 15,000 square degrees of the sky will have been imaged to magnitude $i_{A B} \sim$ 24-24.5, this will translate into approximate 1.5 billion galaxies. Spectroscopy: high resolution spectroscopy will exists for about 25 million galaxies and about 1 million QSO's up to magnitude of about $i_{A B} \sim 22.5$. Also low resolution Near Infrared Spectroscopy will exists for 50 million galaxies. Due to current technological limitations spectrographs can only measure about $10 \%$ of the galaxies.

The decade after next will see a substantial increase in the amount of imaged objects. LSST will image 20,000 square degrees of the sky up to magnitude 27.5, giving 10 billion galaxies. Doing high-resolution spectroscopy for a large fraction of these objects even if only up to magnitude 24.5 will require to increase the number of photons reaching current spectrographs by a factor of 40 and increasing the number of fibers by a factor of 30 . The prospects of doing this are quite daunting. Are there other possibilities? 


\subsubsection{Proposed New Technology}

While high-resolution spectroscopy is highly desirable in many circumstances it may not be really necessary. Medium resolution spectroscopy (e.g. $R=\lambda / \Delta \lambda \sim 80$ ) is enough to get a redshift error of $\sigma \sim 0.003(1+z)$ which translates into an error in distance measurement of about $10 \mathrm{Mpc}$. Going beyond this distance scale runs into the problems associated with the difficulties of making accurate prediction in the non-linear regime of GR.

MKIDs offer the theoretical possibility of achieving $\mathrm{R}$ values of about 80 over a large range of wavelengths. Their small size (100-400 micrometers square) also makes them easy to package in large quantities. In a configuration in which a mask images one galaxy per pixel (see B. Mazin et al, SPIE Proceedings, July 2012) an MKID array of 100,000 pixels will be enough to obtain medium resolution spectroscopic information for all LSST galaxies up to magnitude 24.5. Arrays of 2000 pixels have been built already and there are no known technical problems to increase the size of these arrays by a factor of 500 . The energy resolution of MKIDs has not yet achieved the theoretical limit, $R \& D$ in this area is on going. If in the next several years the energy resolution of these devices can be brought close to the theoretical limit, MKIDs hold the promise of being the only devices capable of providing distance measurements for most of the LSST objects with an error of about $10 \mathrm{Mpc}$ up to magnitude 24.5. 


\title{
Can Columnar Recombination Provide Directional Sensitivity in WIMP Search?
}

\author{
David Nygren \\ Physics Division \\ Lawrence Berkeley National Laboratory
}

\begin{abstract}
A robust signal for sidereal anisotropy in nuclear recoils would support, perhaps more decisively than any other evidence, a discovery claim for a WIMP component of Dark Matter. I present a concept based on columnar recombination in dense xenon gas, to sense recoil direction relative to a TPC drift field. The central advance is the sensing of nuclear recoil directionality information through a comparison of ionization and recombination signals that are produced prior to drifting the track ionization. Conventional track visualization is unnecessary. Restrictions present in contemporary techniques that limit gas density and drift length, and hence total active mass, do not apply here. The optimum xenon density may be near $\rho \approx 0.05 \mathrm{~g} / \mathrm{cm}^{3}$, about ten bars. A monolithic room temperature xenon gas Time Projection Chamber at the ton-scale may be feasible, with unprecedented sensitivity for both directionality and cross-section. Remarkably, the desired operating conditions for $0-v \beta \beta{ }^{136} \mathrm{Xe}$ experiment may be identical, even for a ton-scale system.
\end{abstract}

\section{Introduction}

An attractive hypothesis for Dark Matter (DM) is the Weakly Interacting Massive Particle (WIMP) [1]. As a remnant of Big Bang era, WIMPs must have interaction strength characteristic of weak interactions, although WIMP mass and interaction strength are not strongly constrained by theory. WIMP masses might lie in the range of normal heavy nuclei, although some interpretations of data have suggested masses below $10 \mathrm{GeV} / \mathrm{c}^{2}$ [2]. WIMP DM gravitationally captured in our galaxy would have a truncated virial mean velocity of $\sim 230 \mathrm{~km} / \mathrm{s}$, and is regarded as unlikely to co-rotate with the galactic plane [3, 4]. Given earth's position in the galactic arm, terrestrial detectors co-add a velocity of $\sim 220 \mathrm{~km} / \mathrm{s}$ to a collision. A substantial anisotropy would thereby be induced in the distribution of nuclear recoil directions. The discovery of sidereal anisotropy in nuclear recoils would provide strong, arguably compelling, evidence for the WIMP nature of DM. At present, however, there is no compelling basis that DM is predominantly WIMPs or that WIMPs must have any of these properties [5]. As yet, there exists no widely accepted evidence of their presence at earth. 
In the standard WIMP scenario, the expected nuclear recoil spectra fall rapidly with energy. Such events, typically not more than a few tens of $\mathrm{keV}$, lie well within the range of abundant and nasty backgrounds due to radioactivity and cosmic radiation. Despite these challenges, experimental limits on interaction cross-section versus WIMP mass have been pushed steadily and impressively lower [6].

Solid evidence of directionality relative to a WIMP "wind" would be, arguably, the most robust signature of the WIMP nature of DM, and in my view is the essential step to a claim of discovery. In the event that evidence for nuclear recoils is obtained that cannot be explained with known processes, then the search for directionality in such nuclear recoils will become of immediate and paramount interest. It seems prudent to prepare for this eventuality.

To date, all approaches pursued for directionality sensitivity attempt to obtain some useful measure of nuclear recoil trajectory after drifting an ionization image of the nuclear recoil to a measurement plane with avalanche gain [7-11]. The degrading effects of diffusion during drift, avalanche gain, and visualization/reconstruction noise can only be overcome by increasing the range of nuclear recoils through the use of a dilute gas target and the imposition of a maximum allowable drift length to constrain diffusion. These requirements limit the volume to $\sim 1 \mathrm{~m}^{3}$ or less, and active mass per detector to $\sim 100$ grams. In contrast, non-directional direct detection detectors now in operation or commissioning stages utilize hundreds of $\mathrm{kg}$ - most popularly liquid xenon (LXe) - and tons are contemplated for the very near future. Between detectors without directional and those with directional sensitivity, a difference of at least three orders of magnitude in active mass exists; how can this gap be confronted?

A scheme that combines a large, monolithic, active mass with sensitivity to WIMP wind directionality would hence be most welcome. None have been previously conceived, discussed, or proposed, to the best of my knowledge. Here, I propose a scheme in which all information is collected in the form of optical signals, based on a high-pressure xenon gas electroluminescent (EL) TPC with a special (possibly unique!) molecular additive.

The fundamental notion is that sensitivity may exist in these processes to the "angle" between the nuclear recoil and the TPC drift electric field. The recoil is never "visualized" in the usual sense, yet the angle between track and field may be measurable. The idea is surely speculative, but plausible at each step. If feasible, a remarkable advance in WIMP astronomy will become within our reach. The uncertainties appear addressable by straightforward experimental tests. I elaborate the basic aspects next, followed by a section confronting details as well as the challenges ahead that could lead to a positive demonstration. The concept depends on exploitation of a set of sequential atomic-molecular processes that convert the original track image of excited xenon and xenon ions into a molecular ion image. 


\section{Recombination: a nuisance - or maybe not!}

Can recombination, an ordinary process, be exploited to sense directionality in a large monolithic detector? The answer may be: yes. Recombination is the capture by an ion of an electron freed by an energetic ionizing particle. Capture is likely when an electron wanders sufficiently near an ion; the relevant distance is the Onsager radius, defined as $r_{0} \equiv e^{2} / \varepsilon \mathcal{E}$ [12]. $r_{0}$ is the distance between a positive ion and a free electron for which the potential energy (negative) is balanced by the electron's kinetic energy $\mathcal{E}$. $\varepsilon$, the dielectric constant of the medium, incorporates polarization effects. To set the scale for a thermalized electron with kinetic energy $\mathcal{E}=\mathrm{kT}\left(300^{\circ} \mathrm{K}\right)$ in xenon gas, $\mathrm{r}_{0} \approx 70 \mathrm{~nm}$; for $\mathrm{LXe}, \mathrm{r}_{0} \approx 54 \mathrm{~nm}$, decreased by the polarization of dense xenon. The Onsager radius is well defined for any subexcitation energy, decreasing inversely for increasing electron energy.

Collisions are purely elastic for sub-excitation electrons in pure xenon gas. Fractional energy losses or gains are about $1 \times 10^{-5}$ in each collision, so tens of $\mu \mathrm{s}$ may be required to approach kT. The thermalization distance is hence much larger than the Onsager radius in pure xenon gas.

Recombination processes may be conveniently classified as geminate, ambipolar, diffuse (volume), or columnar. ${ }^{1}$ Geminate recombination has occurred when a "parent" ion regains its own ejected "daughter" electron. For initially hot electrons in pure xenon, geminate recombination is, however, quite unlikely.

Ambipolar diffusion refers to the case of highly localized, strongly ionized, but more or less neutral overall plasma, within which the collective field of the slowly moving positive ions temporarily suppresses escape by diffusion of most of the electrons. Depending on nuclear recoil energy and gas density, ambipolar diffusion is expected play an important role in this scenario.

Diffuse or volume recombination occurs when, in the absence of a strong drift electric field and after a sufficient time, the plasma has become weakly ionized. Ions and electrons created in the event have lost spatial structure. Encounters between electrons and ions become random, and some electrons and ions fail to recombine.

Finally, Columnar recombination (CR) refers to enhancement of recombination when a more or less "straight" ionizing track lies approximately parallel to an external electric field; the field must be strong enough to induce a well-behaved drift velocity, but not so strong that electrons gain energies well above thermal. In this special case, ions and electrons created along the path of the ionizing particle are much more likely to pass near one another repeatedly, increasing the probability of recombination relative to the case of the same electric field perpendicular to, or at a

\footnotetext{
${ }^{1}$ A useful reference is the book "Noble Gas Detectors" by Aprile, Bolotnikov, Bolozhdynya, and Doke [22]
} 
large angle to the track. As in geminate recombination, thermal electrons falling within the Onsager radius of an ion are captured. By definition, columnar recombination requires the presence of an electric field strong enough to overcome thermal agitation of electrons - but not overly strong. It is only columnar recombination that may provide us a signal of nuclear recoil directionality.

The slowly moving nuclear recoils are generally thought to produce many more xenon excitations than ionizations, but I am aware of no data for gaseous xenon to support this assertion. While the population of excitations carries a large fraction of the total energy deposited, the primary excitations carry no directional information. However, in this scenario, the population of excited xenon atoms is converted into a new population of molecular ions and electrons, which then do contribute to directional sensitivity. The new population substantially enhances statistical precision of the two signals that provide directional sensitivity.

\section{What is "Columnarity", and can it be optimized?}

When the average distance between single ionization events is about equal to the Onsager radius, the atomic density could be described as "matched" to the average ionization density. In an average sense, "Onsager spheres" then connect the track segments continuously, i.e., as in a string of beads. In this idealized case of averaged continuous connectivity of the Onsager spheres, the recoil track attains a maximum length. The optimum for sensing directionality of recoil, however, is likely not this simplest case. The aspect ratio $\mathcal{R}=d / \mathrm{r}_{0}$, where $d$ is some measure of nuclear recoil track range, provides a useful geometrical measure of columnarity. For any given recoil energy we might say that "columnarity" is optimized for the largest track length (determined by atomic density) that maintains both a substantial level of Onsager sphere overlap and a large track length. However, for a given energy loss, this simple geometric perspective obviously cannot be complete, as retention and focusing of the electron cloud by the collective positive charge of the ion column is stronger as atomic density increases, and seems sure to be an important factor.

For a $30 \mathrm{keV}$ nuclear recoil in xenon gas for $\rho=0.05 \mathrm{~g} / \mathrm{cm}^{3}$ (about ten bars), the nuclear recoil range is about $2100 \mathrm{~nm}$ (see below). The aspect ratio $\mathcal{R}=\delta / \mathrm{r}_{0}$ is approximately 30 in this case. For comparison, the range in LXe for a $30 \mathrm{keV}$ nuclear recoil track is about $50 \mathrm{~nm} ; \mathcal{R}=\delta / \mathrm{r}_{0} \approx 1$, and Onsager sphere overlap is complete. No electrostatic sense of directionality remains in LXe, and diffuse recombination dominates. Beyond these simple geometric considerations concerning electrostatics, conduction band effects also exist in LXe, delocalizing charge carriers and introducing (unwanted here) high electron mobility.

But how much overlap might optimize a columnar recombination signal? With $\mathrm{r}_{0}=$ $70 \mathrm{~nm}$ in xenon gas, the minimum $\mathrm{dE} / \mathrm{dx}$ for Onsager spheres to "touch" is $\sim 14$ electron/ion pairs per $\mu \mathrm{m}$. As discussed in the next section, the maximum ionization density of $30 \mathrm{keV}$ nuclear recoils in xenon gas at $0.05 \mathrm{~g} / \mathrm{cm}^{3}$ (about ten bars) falls in the range of 100 electron-ion pairs $/ \mu \mathrm{m}$, about seven times the 
geometric minimum $\mathrm{dE} / \mathrm{dx}$. In this case, of substantial overlap, an "Onsager tube" of larger radius is the more appropriate image; the aspect ratio $\mathcal{R}$ is also reduced, but still seems likely to lie above 10 . Overall, perhaps optimistically, if columnarity $\mathcal{R}$ is as high as 10 , a substantial Onsager tube overlap such as seven might be close to an optimum for a recombination signal. Alternatively, if a higher columnarity value turns out to be optimal, gas detectors offer flexibility to vary columnarity widely. Neon can be added at any fraction up to about $90 \%$, the remainder being $10 \%$ xenon + additives as described below. For the same energy, neon recoils in neon travel much further, by a factor of $\sim 15$, with a corresponding reduction in ionization density.

The recoil direction of a WIMP-induced nuclear recoil is best preserved near its origin, when secondary scattering has not yet become appreciable. The first part of the recoil track is also where ionization density of the recoil is highest. The initial track segment defines the optimum atomic density. Detailed simulations including atomic and molecular microphysics are needed to establish the relationships between nuclear recoil energy, xenon density, drift field, and an optimum columnar recombination signal.

Regarding columnarity, it is worth mentioning that $\alpha$ particles differ substantially from nuclear recoils. Even though $\mathrm{dE} / \mathrm{dx}$ may be similar, their track length is orders of magnitude larger, so highly overlapping Onsager spheres do not erase columnarity in this case. Columnar recombination for $\alpha$ particles in gaseous and liquid xenon is expected. The effect degrades energy resolution, but is partially correctable in noble gases by correlation with the scintillation signal of recombination. Furthermore, $\alpha$ particles generate $\mathrm{keV} \delta$-rays, which are absent in nuclear recoils. Measurements of recombination in pure noble gases exist [13], but $a b$ initio calculations appear to be vanishingly rare.

Thus far, the discussion here of electric field, ionization and atomic densities has not included the possible impact of molecular additives that, in general, strongly alter recombination processes and timescales. To realize an optimum columnar recombination signal, I believe molecular gymnastics of a quite special nature will be necessary; remarkably, these may be available to us.

\section{Basic Concept - A columnar recombination signal sensitive to recoil direction}

The essential characteristic of columnar recombination is that a roughly linear event topology lying parallel (or anti-parallel) to the drift electric field leads to more recombination, whereas tracks with sufficiently large angles to the field have relatively much less columnar recombination. Sensitivity to directionality is found through a comparison, for each event, of the recombination "R" signal to the ionization "I" signal. For any given recoil energy, more recombination implies less surviving ionization, and vice versa. 
So the determination of recoil track orientation relative to the drift field shall be made through a comparison, for each event, of the measured columnar recombination population $\mathbf{R}$, to the surviving population of ionization electrons $\mathbf{I}$. The ratio R/I will be larger for nuclear recoil events with a higher fraction of columnar recombination, i.e., those events in which the drift field approximately lines up with (or anti-aligns with) the recoil trajectory.

The ratio $\mathbf{R} / \mathbf{I}$ is the key to sensing directionality. $\mathbf{R} / \mathbf{I}$ may display a soft dependence on total energy, as well as (by design) a prominent dependence on columnar recombination. The total energy $\mathbf{E}$ of the event is a weighted sum: $\mathbf{E}=a \mathbf{R}+b \mathbf{I}$. The coefficient "a" will depend on the spatial variation of detection efficiency of light within the active volume. This spatial variation may be modest, since optical detection is foreseen to closely approach $4 \pi$, as elaborated below. Both coefficients will depend also on whether the event is classified as electron or nuclear recoil. Calibration of coefficients $(a, b)$ for electron recoils is made using $\gamma$-rays of definite energy. For nuclear recoils, calibration of $(a, b)$ will depend on ancillary scattering experiments using neutron sources and simulation; but the resolution requirement for nuclear recoils is not as stringent as for electron recoils.

The ratio $\mathbf{R} / \mathbf{I}$ has an inverse but not exact correspondence to the ratio $\mathbf{S 2 / S 1}$, commonly used in liquid xenon DM searches. ${ }^{2}$ The form $\mathbf{R} / \mathbf{I}$ is chosen simply to take representational advantage of the much smaller fluctuations in the measured $\mathbf{I}$ signal relative to $\mathbf{R}$.

The unique, and powerful, advantage of this scheme is that the topological information contained in $\mathbf{R} / \mathbf{I}$ is extracted before the degrading impact of diffusion during drift confounds topology. Thus, requirements for spatial reconstruction of the ionization image at the readout plane are undemanding and conventional. Thus the detector based on measuring $\mathbf{R} / \mathbf{I}$ should scale gracefully to quite large monolithic masses, since both UV light and ionization can be transported over long drift distances without significant degradation. It seems quite plausible that monolithic ton-scale systems may be realizable with this technique.

The necessary conditions appear to be five:

1. Nuclear recoils retain, in a statistical sense, significant directionality relative to the incoming WIMP direction.

2. The spatial structure of the primary ions and electrons created after the nuclear recoil event collectively retain a sufficiently linear character;

3. The population of atoms/molecules that experience columnar recombination in a typical event is large enough that useful statistical precision exists;

\footnotetext{
${ }^{2} \mathrm{~S} 2$ is the ionization signal, observed optically through electroluminescence after drifting through the active mass into a gaseous region of high electric field; S1 is the primary scintillation, which in LXe displays a decay time of $27 \mathrm{~ns}$, and is the prompt signal that defines the time origin of the event.
} 
4. The population of atoms/molecules that experience columnar recombination can be measured with adequate precision and accuracy;

5. This population can be detected with adequate discrimination against the other classes of recombination.

It appears that all these conditions can be met. Furthermore, it appears that electron recoil backgrounds may produce very different $\mathbf{R} / \mathbf{I}$ values, permitting extremely powerful discrimination.

Both $\mathbf{R}$ and I populations are to be measured through optical (UV) signals sensed at different times. Separation of the ionization signal $\mathbf{I}$ from $\mathbf{R}$ is possible because $\mathbf{I}$ is measured after drift, which provides a substantial time interval between signals.

As the angle between electric field and nuclear recoil direction increases, the level of columnar recombination will decrease. In any case, it is unlikely that the selectivity of columnar recombination will be extremely sharp, for a variety of reasons such as the fact that the electrons occupy a larger "volume" than the line of ions, as well as secondary scattering as the nuclear recoil passes through the xenon.

It is worth noting that the formation of the two signals $\mathbf{R}$ and $\mathbf{I}$ occurs locally in a uniform, completely homogeneous medium, and is unaffected by any granularity in the detection process, as would likely exist in any track visualization technique.

\section{Molecular gymnastics: Charge Exchange \& Fluorescent Penning Molecules}

A novel part of the idea is to combine plausible but less familiar atomic/molecular processes in a way that maximizes sensitivity to columnar recombination and also maximizes a measurable optical signal. In particular, molecules that display efficient UV fluorescence are essential. The candidate molecules may be few, but possibly excellent: the tertiary amines trimethylamine and triethylamine. The optimum molecular fractions may be reasonably expected to fall in the range of 2 percent, but perhaps less or a bit more (see below).

To prepare the discussion, the primary atomic/molecular processes of interest are:

$$
\begin{array}{ll}
\mathrm{Xe}_{\mathrm{NR}}+\mathrm{Xe} \rightarrow \mathrm{Xe}+\mathrm{Xe}+\text { heat } & \text { (loss of energy to elastic scattering) } \\
\mathrm{Xe}_{\mathrm{Nr}}+\mathrm{Xe} \rightarrow \mathrm{Xe}+\mathrm{Xe}^{*} & \text { (excitation) } \\
\mathrm{Xe}_{\mathrm{Nr}}+\mathrm{Xe} \rightarrow \mathrm{Xe}+\mathrm{Xe}^{+}+\mathrm{e}^{-} & \text {(ionization) }
\end{array}
$$

The important secondary processes are:

$$
\begin{array}{ll}
\mathrm{Xe}^{+}+\mathrm{e}^{-} \rightarrow \mathrm{Xe}^{*} & \text { (Recombination) } \\
\mathrm{Xe}^{+}+\mathrm{TMA} \rightarrow \mathrm{Xe}+\mathrm{TMA}^{+} & \text {(Charge exchange) } \\
\mathrm{Xe}^{*}+\mathrm{TMA} \rightarrow \mathrm{Xe}+\mathrm{TMA}^{+}+\mathrm{e}^{-} & \text {(Penning effect) }
\end{array}
$$

The important tertiary processes are TMA recombination and fluorescence:

$$
\mathrm{TMA}^{+}+\mathrm{e}^{-} \rightarrow \mathrm{TMA}^{*} \quad \text { (geminate and non-geminate processes) }
$$


Optimization is defined here as maximizing sensitivity to the columnar process, while, to the highest extent possible, minimizing geminate $\mathrm{TMA}^{+}$recombination, since that process provides no directional signal. On the other hand, the geminate process $\mathrm{Xe}^{+}+\mathrm{e}^{-} \rightarrow \mathrm{Xe}^{*}$ is benign, since the Penning process acts to return that energy to free ionization. I expect that the set of optimum conditions has a gentle character, in that needed control of operating parameters in a large detector is reasonably straightforward to manage.

All of these processes are certain to occur, but their efficiency for the purpose of directional sensitivity depends on poorly understood rates. Specifically, both Penning transfers and charge exchange between xenon ions and TMA must occur more rapidly than the departure of electrons from the evolving ion column. For highly ionizing tracks, at densities for which ambipolar diffusion is prominent, this may be true. However, even if all the primary ionization electrons were to escape, the primary population of excitations is much larger for nuclear recoils, and the Penning effect will quickly create a much larger number of free electrons from this population. This late-appearing population of electrons will be exposed to the converted $\mathrm{TMA}^{+}$ion image and provide the bulk of any directional sensitivity.

\section{A specific application, or a general concept?}

The other noble gas candidates for direct detection WIMP searches, argon and neon, and conceivably helium, each have a kinematic optimum match to WIMP mass. Xenon serves here to illustrate the concept, but xenon + neon mixtures may have an important and straightforward advantage for optimizing columnarity.

At this point, many questions arise: What is the range of atomic density for nearoptimal columnarity? What is the range for near-optimum drift electric field? How does the $\mathbf{R} / \mathbf{I}$ ratio, in principle, vary with field-track angle? How does that sensitivity vary with nuclear recoil energy? How efficiently does the recombination process with TMA convert energy to the desired UV signal? How can columnar recombination be enhanced relative to geminate and diffuse recombination? These questions require new experimental work, likely aided by simulations, to obtain useful answers.

Next, I give an overview of the nuclear recoil event and then discuss how the resulting populations of ions, electrons and excitations may be exploited. Finally, I describe current and future R\&D activities.

\section{The nuclear recoil event - and what happens next}

The basic structure and character of xenon ion trajectories in xenon gas can be visualized with the code Stopping and Range of Ions in Matter (SRIM) [14]. The 
density chosen here for simulation is $\rho=0.05 \mathrm{~g} / \mathrm{cm}^{3}$, about ten bars at room temperature, or $1.6 \%$ of liquid xenon density: $\rho=3.1 \mathrm{~g} / \mathrm{cm}^{3}$.

SRIM is claimed to be accurate to about $5 \%$ for stopping power and range. SRIM displays trajectories without weighting by energy loss. The typical ion range is seen to be about two to three $\mu \mathrm{m}$ in pure xenon. The trajectory "ends" when all particles fall below an ionization threshold, but SRIM does not seem to provide a clear definition of the threshold. Figures 1 and 2 show 200 xenon recoil trajectories with initial energy of $30 \mathrm{keV}$, entering along the X-axis. Although scattering is substantial, the trajectories clearly retain a substantial level of their initial directionality. An event takes less than $\sim 30$ ps from start to finish.

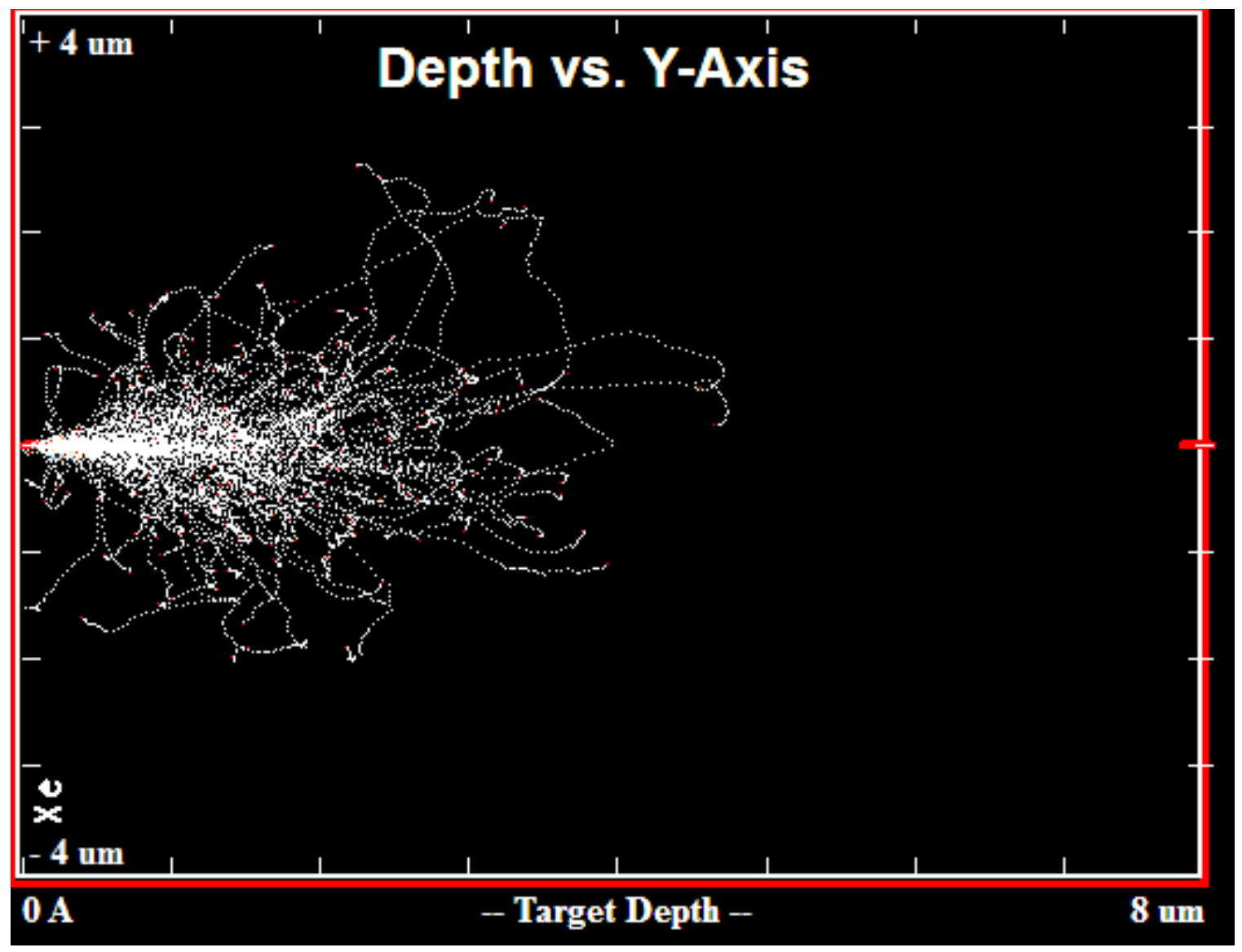

Figure 1. SRIM results for 200 xenon ions of $30 \mathrm{keV}$ entering xenon gas at the left. The xenon gas density is $\rho=0.05 \mathrm{~g} / \mathrm{cm}^{3}$, about ten bars. The trajectories are unweighted by energy loss in this visualization. Although scattering is substantial, the retention of original directionality is seen to be significant, at least qualitatively.

Xenon atom recoils with kinetic energy of $30 \mathrm{keV}$ are well above expected detection threshold and still below form-factor suppression of cross-section in xenon. The initial atom velocity $v_{i} / c$ is about $2 / 3 \times 10^{-3}$. At such a low velocity, atomic shells follow the nucleus and the xenon atom initially recoils coherently [15]. Energy loss 
is greatest at the beginning of the track, where directionality is best preserved. Partial ionization of the outer shell is likely during recoil. Unfortunately SRIM does not provide any measure of fractional energy given to excitation. No measurements of excitation by xenon recoils in xenon gas appear to be available in the literature.

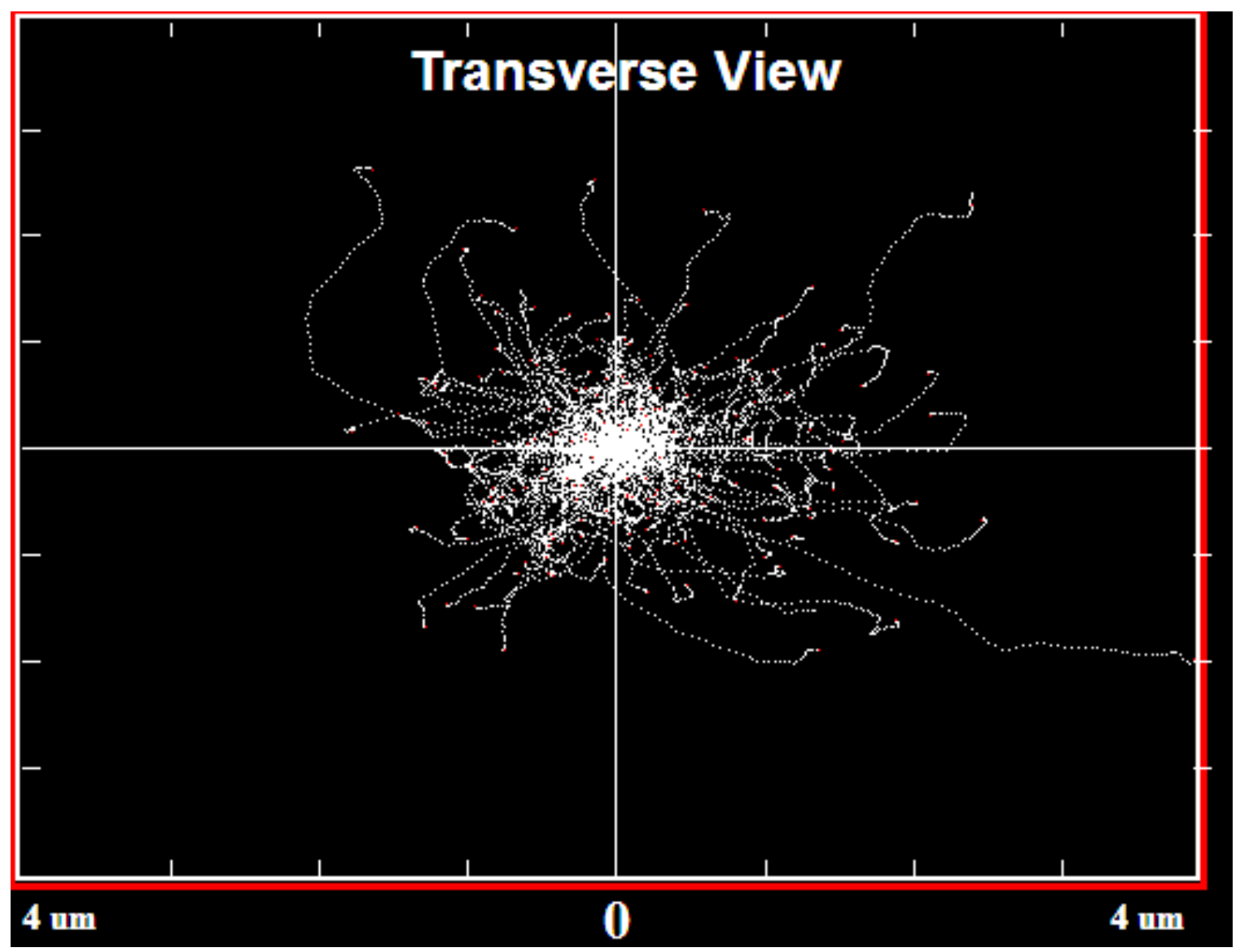

Figure 2. The same 200 xenon ions are shown in transverse view, displaying departures from the initial direction along the X-axis (SRIM coordinates). The xenon gas density is $\rho=0.05 \mathrm{~g} / \mathrm{cm}^{3}$, about ten bars. The trajectories are unweighted by energy loss in this visualization. Relative to the spatial distribution of figure 1, the trajectories are highly concentrated around $\mathrm{Y}=\mathrm{Z}=0$.

An effort is underway to obtain, at least semi-quantitatively, an estimator of "directionality" from the ensemble of trajectories from SRIM. The ultimate goal is an estimator of columnar recombination, to understand and optimize sensitivity to drift field orientation. It is unclear if SRIM is the optimum path toward this goal.

Figure 3 illustrates how ionization event energy is distributed in space. 100,000 xenon ions of $30 \mathrm{keV}$ enter from the left, along the midline of an $8 \times 8 \mu \mathrm{m}^{2}$ plane. The red histogram shows the distribution along the X-axis, revealing the wellknown decline in energy transfer as the ion slows. The green histogram shows the 
transverse distribution in ionization; clearly, the initial segments of the trajectories, where most of the energy is deposited, retain a high level of directionality.

\section{Target lonization}

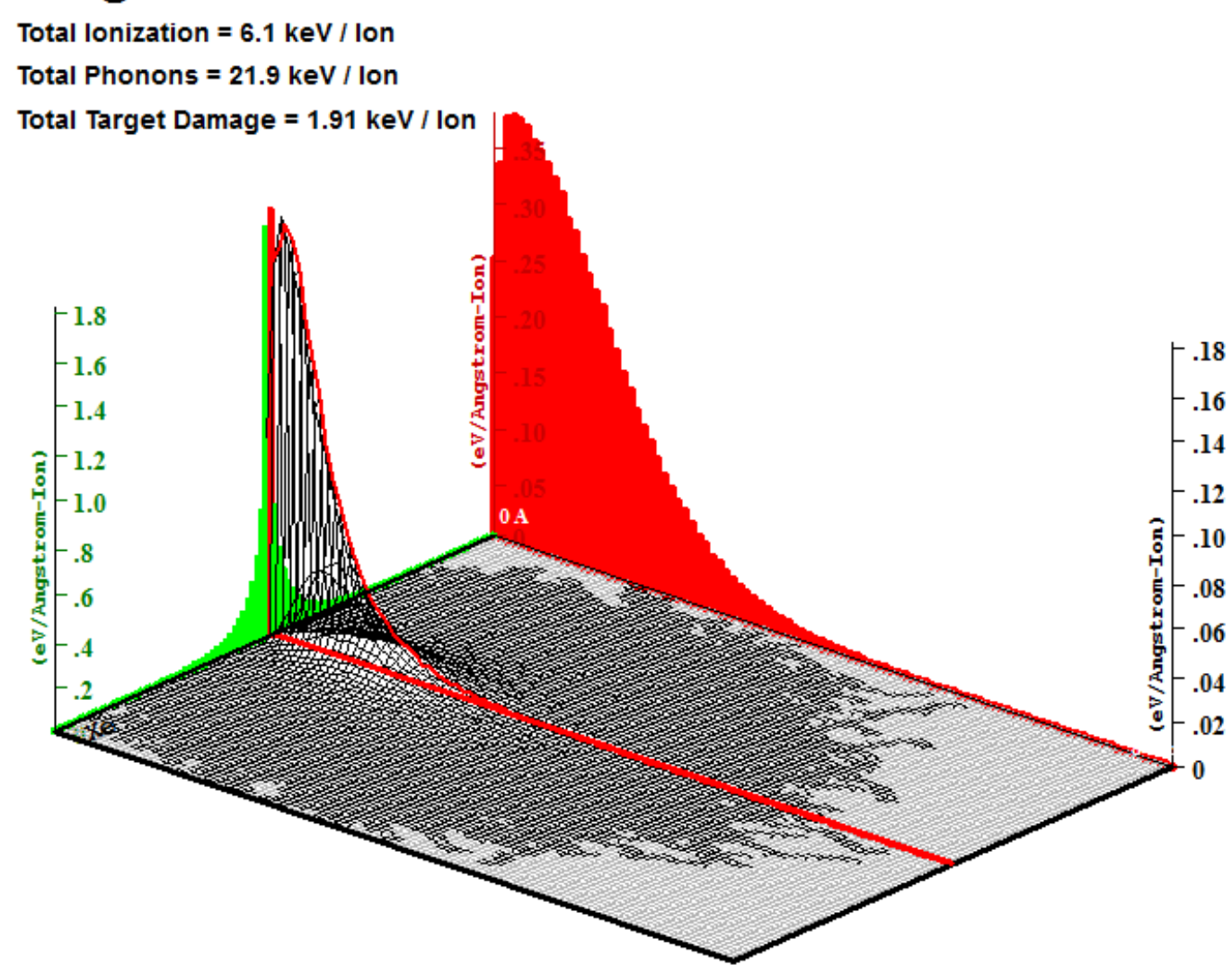

Plot Window goes from $0 \mathrm{~A}$ to $8 \mathrm{um}$; cell width $=800 \mathrm{~A}$ Press PAUSE TRIM to speed plots. Rotate plot with Mouse.

Ion $=\mathrm{Xe}(30 . \mathrm{keV})$

Figure 3. This SRIM plot shows how energy is distributed in an $8 \times 8 \mu \mathrm{m}^{2}$ plane for 100,000 xenon ions of $30 \mathrm{keV}$ entering from the left along the X-axis, the red line. The red histogram displays the deposited ionization along the original ion direction, $\mathrm{X}$, integrated over $\mathrm{Y}$ and $\mathrm{Z}$. The green histogram shows the transverse distribution of ionization - note the spike at $\mathrm{Y}=0$. The grey histogram indicates ionization density in the plane. The xenon gas density is, again, $\rho=0.05 \mathrm{~g} / \mathrm{cm}^{3}$, about ten bars.

At the instant when nuclear recoil is "complete" - i.e., when no further collisions occur - the event consists of a spatially correlated ensemble of free electrons, ions, and excitations. All three of these populations play an essential role in the development of directional sensitivity. The SRIM result for total ionization in figure 3 is $6.1 \mathrm{keV}$. If the average energy to create an ion-electron pair is taken as $25 \mathrm{eV}$, an average of $\sim 240$ free electrons are liberated in a $30 \mathrm{keV}$ nuclear recoil. If the track length of a $30 \mathrm{keV}$ nuclear recoil in xenon at $0.05 \mathrm{~g} / \mathrm{cm}^{3}$ is taken as $\sim 2 \mu \mathrm{m}$, then the ionization density corresponds to $\sim 100$ electron-ion pairs $/ \mu \mathrm{m}$. 
While considerable data for nuclear recoils in liquid xenon (LXe) exist, it cannot be assumed a priori that the ratio of ionization to excitation in HPXe is identical to that of LXe, since in LXe the conduction band delocalizes carriers, strongly affecting recombination and carrier velocities [16]. For the gas densities considered here, conduction band effects are negligible. At room temperature, the impact of density approaches dominance only for densities greater than $\rho \approx 0.55 \mathrm{~g} / \mathrm{cm}^{3}$ [17].

Even at thermal energies, electron speed is remarkably high, about $v_{i} / c \approx 1 / 3 / x 10^{-3}$, or $100 \mathrm{~mm}$ per $\mu \mathrm{s}$. The velocity of $30 \mathrm{keV}$ xenon ions, $\mathrm{v}_{\mathrm{i}} / \mathrm{c} \approx 2 / 3 \times 10^{-3}$, is only twice the speed of thermal electrons. Ions of a few tens of $\mathrm{keV}$ experience soft collisions only and cannot produce $\delta$-rays. However, the primary nuclear recoil can produce relatively energetic secondary atomic ions, leading to complex topologies.

As the response of xenon is strongly affected by the presence of molecular additives, measurements of both electron and nuclear recoils are needed under a range of operating conditions. To summarize, nuclear recoils clearly retain high levels of their original directionality, at least initially. The detailed event histories in SRIM may provide a basis for further assessment, but simulation of ambipolar effects is yet to be undertaken.

\section{After nuclear recoil: electron thermalization, drift and recombination}

Diffusion begins even before the recoiling atom has lost its energy. Free electrons generated during the event thermalize quite rapidly in the presence of complex molecules but very slowly if in pure xenon. The dense positive ion column collectively hangs on to the ensemble of electrons, even as diffusion leads some peripheral electrons to depart permanently while relentlessly assisting the ion column to capture other electrons.

In the presence of an electric field large enough to dominate the collective behavior of the electron ensemble, i.e., a drift field, recombination is enhanced if the drift field is nearly parallel to the ion column, causing electrons to move past it, i.e., promoting columnar recombination. In contrast, if the ion column is not aligned with the drift field, recombination is reduced as the electrons move more transversely. Thus the fraction of electrons experiencing columnar recombination is a measure of the relative orientation of the drift field and recoil nucleus trajectory.

An electric field can of course be too small to overcome diffusion, and is thus not a "genuine" drift field. Geminate recombination can be substantial in that case if molecular additives are present. As electric field is increased from zero, the transition between diffusion-dominated and genuine drift regimes can be surprisingly abrupt. On the other hand, if the drift field is too large, electrons gain significant energy (even in the presence of complex molecules), and recombination is suppressed. The important conclusion here is that an optimum drift electric field value "Eopt" likely exists, minimizing geminate and diffuse recombination while 
maximizing sensitivity to columnar recombination for tracks parallel to the field. $\mathrm{E}_{\text {opt }}$ will likely depend linearly on the fraction of molecular additive.

An impact of density appears through $\sigma^{2}=2 \mathrm{DT}$, where $\sigma^{2}$ is the spatial variance (not necessarily the same in directions parallel to drift and transverse to drift), D is the diffusion coefficient, and $\mathrm{T}$ is elapsed time. In the presence of a moderate drift field, $\mathrm{T}=\mathrm{L} / \mathrm{V}$, where $\mathrm{L}$ is the drift length and $\mathrm{V}$ is electron drift velocity. The diffusion coefficient $\mathrm{D}$ is defined as $\mathrm{v} \ell / 3$, where $\mathrm{v}$ is mean electron speed, and $\ell$ is the mean length between electron-atom/molecule collisions; $\ell$ is proportional to $1 / \rho^{1 / 3}$. Thus, for fixed electron temperature, $\sigma^{2} / \mathrm{L}$ is proportional to $\rho^{-1 / 3}$, a gentle dependence. While the initial ensemble of ions and electrons does not display clues dependent on density, a density dependent effect emerges quickly with time, but is modest.

With low drift fields present in pure xenon, electrons gain energy from the field but lose only a tiny fraction in any elastic collision. In "drift" regime, the average energy of electrons can increase to a few $\mathrm{eV}$, but electrons never reach the first excitation potential of atomic xenon, $\mathrm{E}_{\mathrm{exc}}=8.315 \mathrm{eV}$. The absence of dissipative collisions leads to a high level of diffusion, but backward scattering limits the collective electron drift velocity $\mathrm{V}$ to only $\sim 1 \mathrm{~mm} / \mu \mathrm{s}$. Diffusion in pure noble gases is, consequently, unpleasantly large for any conventional drift field value.

Molecular additives, even relatively simple ones such as $\mathrm{N}_{2}, \mathrm{CH}_{4}$ or $\mathrm{CF}_{4}$, radically alter electron transport properties in noble gases, even at levels as low as $0.01 \%$. Collisions with complex molecules cool electrons dramatically. Diffusion is reduced, in some cases quite strongly, and the drift velocity relative to pure noble gas is increased. To maximize columnar recombination, the drifting electrons should clearly be kept near thermal energies and the drift velocity low. It is unclear at present what the electron drift velocity should be, but likely should remain close to $1 \mathrm{~mm} / \mu \mathrm{s}$ or $1 \mu \mathrm{m} / \mathrm{ns}$. This is well below the initial velocity of $\sim 200 \mathrm{~mm} / \mu \mathrm{s}$ for a 30 $\mathrm{keV}$ xenon recoil. But as thermal electron speed is similar to that of the nuclear recoil, will electrons be well constrained by the collective field of the positive ions?

To summarize, for a small drift field, complex molecular additives cool electrons, increase drift velocity significantly, and reduce diffusion by large factors, relative to pure xenon. However, as elaborated below, two additional molecular properties are desired: a strong Penning effect in xenon and efficient UV fluorescence in a specific band. Which molecules, if any, might then serve best? In the scenario developed below, trimethylamine (TMA), or triethylamine (TEA) or perhaps even dimethylamine (DMA), is added at the few percent level, probably around $2 \%$.

The desired combination of properties is a strong Penning effect in xenon and efficient fluorescence in a band around $300 \mathrm{~nm}$. TMA and DMA appear to display a strong Penning effect, while TMA and TEA are known to fluoresce strongly in a narrow band around 300nm $[18,19]$. However, it could be true that DMA fluoresces too and that TEA also displays a strong Penning effect - the needed measurements 
simply have not been done or published. I use TMA generically in what follows to represent any of these amines unless specifically noted.

\section{Xenon excitations: Penning effect molecules convert excitations to ionization}

In the xenon/TMA gas, an event originated by an energetic electron or nuclear recoil produces a track with ionized and excited atoms/molecules. The ratio of primary excitation/ionization in dense xenon gas is known with useful accuracy for electron recoils, but remains unmeasured for xenon nuclear recoils in xenon gas. It is reasonably expected, however, that nuclear recoils in xenon gas will produce more primary excitations relative to ionizations, whereas the reverse is true for energetic electron recoils. The molecular gymnastics I invoke next profoundly alter this simple picture.

The Penning effect can occur in noble gas mixtures with a secondary component that has an ionization potential (IP) slightly smaller than the primary noble gas first excitation energy. The long lifetime of the noble gas first excited state permits an efficient transfer of energy to the additive, resulting in conversion of noble gas excitation to ionization of the additive (not all molecular additives display this effect, even if their IP is a good match). Ionization is relatively easy to detect and measure, e.g., in a TPC, and in a TPC with an EL gain stage, ionization can be measured very accurately [20]. An EL TPC is a very natural application also for a $0-v$ $\beta \beta$ decay search in ${ }^{136} \mathrm{Xe}$ as well as for a WIMP search [21].

As noted, the primary population of ions and electrons is, of course, reduced by any of the recombination processes. In the pure noble gases, both recombination and excitation normally lead to detectable radiation in the VUV, characteristic of relaxed excimers [22]. In pure xenon this signal appears in a band centered at $173 \mathrm{~nm}(7.3$ $\mathrm{eV})$ with a width of about $12 \mathrm{~nm}(\sim 1 \mathrm{eV})$. The $173 \mathrm{~nm}$ wavelength is unpleasantly deep into the VUV, imposing some difficulties and limitations for detection. Furthermore, since primary excitation is insensitive to topology, any direct "prompt" UV signal contribution from it would dilute the recombination signal we seek. It is essential to transform the population of primary excitations to ionization, which only then may contribute to recombination. Use the Penning effect to convert all primary xenon excitations to ionization.

For simplicity, I shall assume that the Penning process is $100 \%$ efficient although this assumption is neither certain nor required. Not many molecular species are known to display a Penning effect with xenon. Ramsey and Agrawal [23] presented results suggesting that both trimethylamine (TMA) and dimethylamine (DMA) display a strong Penning effect with xenon at $\sim 1$ bar. Their experiment used a conventional cylindrical proportional chamber, measuring gain versus additive concentration and HV. The evidence in [23] for a strong Penning process is plausible, but indirect: strongly increased gain for TMA and DMA at a given voltage, relative to other similar molecular additives with IPs somewhat larger than the excimer energy of $\sim 7.3 \mathrm{eV}$ or the atomic excitation energy of $8.315 \mathrm{eV}$. Further indirect evidence that supports the presence of a strong Penning effect in xenon- 
TMA mixtures, has been recently reported [24] [http://arxiv.org/abs/1210.3287]. In this important work, the Penning effect appears maximized between 1.5 and 3\% TMA, and exhibits a modest variation with atomic density up to at least ten bars.

More direct evidence, such as a higher primary ionization yield, and/or a directly measured reduction of the Fano factor would be valuable, but I have found no evidence in the literature for either of these more basic effects in xenon gas + molecular additives. Reported values for the ionization potential (IP) of molecules like TMA, DMA, and TEA vary considerably according to technique. The NIST Chemistry WebBook [25] offers a precise value for the IP of TMA: $7.85 \pm 0.05 \mathrm{eV}$. A newer, precise value for the IP of DMA is $8.32 \pm 0.03 \mathrm{eV}$ [26] - equal within error to the first excitation energy of xenon. It is conceivable that the Penning effect in DMA is exactly resonant with excited xenon atoms $(8.32 \mathrm{eV})$. In xenon + TMA the Penning effect is not fully resonant (unless the IP value is in error), but is perhaps facilitated by less geminate recombination due to the slight excess electron energy.

For xenon + triethylamine (TEA), Ramsey and Agrawal [23] did not observe increased gain, relative to xenon + TMA, although xenon + TEA was not far away.

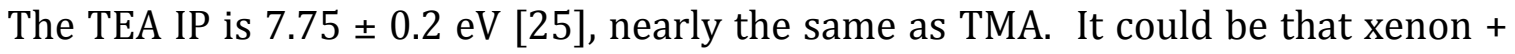
TEA is also a good Penning mixture, but that TEA is much better than TMA at cooling electrons, requiring greater voltages to reach the same gain as xenon + TMA. So the situation remains unclear which molecule, TMA or DMA - or possibly TEA or even a yet unrecognized molecule - might be best as a Penning molecule for xenon.

DMA is not a symmetric molecule, and although it might be expected to display negligible electronegativity, none was evident in [23]. For simplicity, I assume here that the tertiary aliphatic amines, TEA and TMA, are the molecules of interest, but DMA is not ruled out.

I further assume that all xenon excitations are transformed by a Penning effect into a proportional contribution to ionization. This assumption is not completely safe; TMA/TEA appear to have a photo-dissociation threshold around $5 \mathrm{eV}$ [18]. Any free $8.315 \mathrm{eV}$ VUV photons from excited atomic xenon, as well as any $\sim 7.3 \mathrm{eV}$ photons from xenon excimers, will be strongly absorbed in an encounter with TMA. Without direct evidence that suppression of xenon VUV by the Penning effect is $100 \%$ efficient, I shall assume that the TMA molecule experiences ionization rather than dissociation.

For $\rho \approx 0.05 \mathrm{~g} / \mathrm{cm}^{3}$ the xenon-xenon atomic collision rate is only about $10^{11} \mathrm{~s}^{-1}$, so, for a TMA concentration of $1-3 \%$, the collision rate for TMA (a larger molecule) with $\mathrm{Xe}^{*}$ is correspondingly less, only a few $\mathrm{GHz}$. Penning conversion of $\mathrm{Xe}^{*}$ to $\mathrm{Xe}^{+}+$ $\mathrm{e}^{-}$will hence likely be complete in about one ns, perhaps less. However, unless ambipolar diffusion prevails, most of the free electrons generated by primary ionization will be swept past (or away from) the ion column in a fraction of one ns. 
Some columnar recombination with $\mathrm{Xe}^{+}$may occur for those electrons that happen to reach near-thermal energies. The resulting $\mathrm{Xe}^{*}$ excitations are candidates for recycling to free ionization by Penning conversion. It is essential, of course, that some electrons escape, in order to provide an I signal. But the quantity of these first escapees may be smaller than a secondary component provided by Penning conversions in a larger population of excitations.

Conversion of the excitation population to a contribution to ionization reduces the Fano factor. This is beneficial to the resolution of energy measurement by ionization signal. For pure gaseous xenon, $\mathrm{F}=0.15 \pm 0.03$ [27]. Perhaps, if a strong Penning effect for xenon exists with TMA, the Fano factor might reduce to 0.08 or conceivably even lower, benefiting the neutrino-less double beta decay search in ${ }^{136} \mathrm{Xe}$. In any case, I assume here that the optimum fraction of TMA for any xenon density is to be found experimentally. The optimum xenon density may be quite broad since no scale exists until conduction band effects enter prominently above $\rho$ $\approx 0.55 \mathrm{~g} / \mathrm{cm}^{3}$. As noted, the optimal fraction of TMA might be around $2 \pm 1 \%$ [24].

\section{Xenon lons: neutralization by charge exchange with TMA}

At the instant following the event, a nearly immobile image of the event exists in the form of xenon ions, plus the much smaller ensemble of ionized molecular additive. An agitated shell of electrons surrounds the ions. Some geminate xenon recombination occurs, but thanks to the Penning effect, the resultant excited atoms and/or excimers are promptly returned back to the ionization population.

An efficient charge exchange process with TMA neutralizes ionized xenon atoms, since the $12.14 \mathrm{eV}$ IP of xenon is much higher than the IP of TMA or any other of the amines. For $\rho \approx 0.05 \mathrm{~g} / \mathrm{cm}^{3}$ and a TMA concentration of $1-3 \%$, the collision rate for $\mathrm{Xe}^{+}$with TMA is a few $\mathrm{GHz}$, presumably the same rate as $\mathrm{Xe}^{*}$ with TMA. Optimistically, the charge exchange conversion process may be complete in about one ns. The neutralized but excited xenon atoms are now candidates for further Penning transfers. I assume that all xenon excitations, primary or recombinant, will be converted to ionization, and that the initial xenon ion track image is replaced by a nearly exact image of amine ions plus a matching set of new, secondary electrons.

The ionic charge exchange process may be enhanced by resonant charge exchange (RCE) [28]. RCE is a quantum mechanical phenomenon observed in pure or nearly pure gases that enables rapid transfer of charge between identical particles since no energy barrier exists. Thus RCE, by increasing the diffusion of charge among nearby xenon atoms, may increase the effective rate of encounters between ionized xenon and TMA. Ionized xenon dimers also form, as well as evanescent clustering, yielding a variety of partially solvated xenon ions. The impact of clustering and solvation is unclear. It might accelerate charge exchange by opening up more phase space for momentum transfer among participants, or conversely, perhaps solvation introduces shielding by polarization, impeding charge exchange. 
The ionization electrons from nuclear recoil are nearly all sub-excitation in energy, unlike those from energetic electron recoils [29]. As noted, the electrons lose negligible energy in collisions with xenon, but after one or a few encounters with amines, all electrons reach some quasi-thermal energy distribution determined by drift field, density, and molecular composition. Ambipolar diffusion is presumably dominant.

The relative angle of recoil and drift field has no impact for the ionic charge exchange process; noise introduced is negligible. The ionic charge exchange process is likely to occur in a single encounter. The thermalization time scale for electrons is plausibly longer than the timescale for ionic charge exchange, even though the average velocity of electrons is much higher than that of ions. But even if this is not true, ambipolar diffusion may retain most of the electrons until ionic charge exchange is complete. Some density dependence likely enters here.

\section{The Recombination Process!}

At this point, the Penning effect and charge exchange has ensured that all ions are amines. $^{3}$ The columnar recombination process with amine ions can now begin to play its role. The electron population is composed of the original ionization plus a secondary contribution from Penning conversions. The optimal drift electric field will minimize quasi-geminate and diffuse recombination, both unwanted processes that blur directional sensitivity, while maximizing columnar recombination.

The use of a magnetic field parallel to the drift field, as in the traditional TPC scenario, could be considered to enforce proximity of the electron population to the original ion column. There could be some benefit here, but the Ramsauer-Townsend minimum in the electron-xenon cross-section is not nearly as deep as it is for argon, and the minimum is near $1 \mathrm{eV}$, rather than near-thermal as in the case of argon. The suppression of transverse diffusion might be much less than desired, especially considering the cost of realizing a strong solenoidal field deep underground.

\section{The Fluorescent Penning Molecule provides the Recombination Signal R}

In order to extract information from the columnar recombination population, we must have not only a Penning molecule that transforms the unwanted xenon excitations to the ionization population, but also a way to sense the emergent columnar recombination population. The solution is to use a Penning molecule that fluoresces efficiently in recombination, thereby providing a useful optical signal.

While this may seem unlikely, it turns out that, remarkably, TMA and TEA are likely to be efficient Fluorescent Penning Molecules (FPM). Cureton et al [18] show that

\footnotetext{
3 It may be useful to define "quasi-geminate" recombination, referring to an electron captured by the amine ion created by charge exchange with the xenon ion that lost that particular electron. Some of this will surely occur, and it contributes nothing to the directionality signal.
} 
TMA and TEA fluoresce in a narrow set of bands spanning $275-330 \mathrm{~nm}$ (see figure 4), and with unitary efficiency, at least when stimulated by $\sim 240 \mathrm{~nm}$ UV photons.

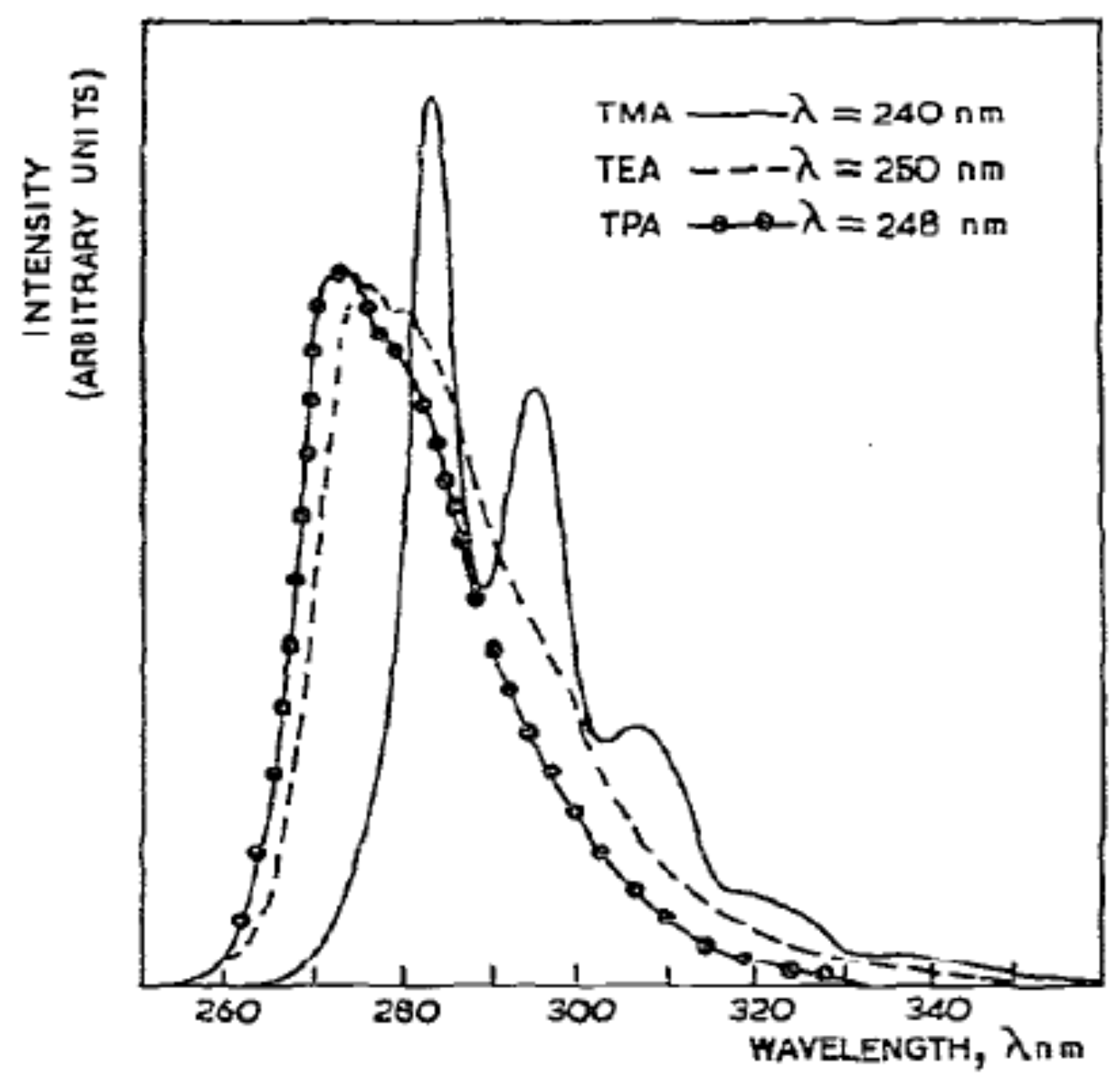

Figure 4. Vapor-phase fluorescence spectra of TMA, TEA, and tripropylamine (TPA) excited at the indicated wavelengths; from [18].

Cureton et al. observed short (1 - $8 \mathrm{ns)}$ and long (40 - $50 \mathrm{ns)} \mathrm{fluorescence} \mathrm{decay}$ time constants in both TEA and TMA, with relative yields depending strongly on excitation wavelength. Although these decay time constants are long relative to other processes of concern here, they refer to processes after recombination has taken place, and no adverse impact on the desired $\mathbf{R}$ signal is expected. This work used pure amines at very low pressures in the 0.1 to 3 milli-Torr range, to minimize collisional quenching and line broadening,

Kreuger et al. [19] examined light emerging from a GEM in xenon-TMA over a range of 1 to 8 bars, and observed an optimum of about 2.5\% TMA for gain. Kreuger et al. further observed spectral features in the presence of dense xenon gas that resemble closely those of figure 4 here, with little variation with density, and that the light 
yield per secondary electron depends only slightly on the TMA concentration. This important work provides further confidence that the desired fluorescence features will indeed be present in high-pressure xenon gas within a range of at least $1-3 \%$ TMA. This work also bolsters the claim of Ramsey and Agrawal [23] that a strong Penning effect is present in xenon-TMA.

Charpak et al showed that in an appropriate electric field, TEA + argon fluoresces brightly at one bar, indicating that energy transfer from energetic electrons of $\sim 4 \mathrm{eV}$ leads to fluorescence, i.e., electroluminescence [30]. Regrettably, that work did not provide quantitative results for efficiency. However, the presence of EL in the absence of charge multiplication over a finite range of E/p (EL electric field divided by gas density) is an extremely important result from that work, showing that no electrons reach the IP of TEA (IP $=7.75 \mathrm{eV}$ ) over a limited but adequately finite range. The same result is to be reasonably expected for xenon - TEA, and plausibly, also for xenon-TMA, since these molecules have almost identical IPs.

Quite providentially then, we have at least one, possibly two, Fluorescent Penning Molecular candidates, TMA and TEA (and maybe DMA), for high-density xenon gas. Even more remarkable, the $300 \mathrm{~nm}$ range of fluorescence appears to be a nearperfect match to detection with wavelength-shifting plastics, as elaborated below.

\section{The Detection of $\mathbf{R}$ and I Signals}

\section{Detection of R: Maximize Optical Detection Efficiency for 300 nm UV}

In order to preserve as much statistical precision in the measured ratio of $\mathbf{R} / \mathbf{I}$ as possible, it is clearly essential to realize the highest possible optical detection efficiency for this $285-310 \mathrm{~nm}$ range. Xenon is completely transparent at $300 \mathrm{~nm}$. TMA and TEA are also transparent to their own fluorescence, according to the most natural interpretation of the results in $[18,19]$. Even if reabsorption by TMA-TEA is significant, re-emission at a slightly longer wavelength should be beneficial or at very worst, benign - if excitation is as efficient as reported in [18].

Naively, one might consider covering the entire surface of the detector with PMTs to maximize optical detection efficiency, as shown in some preliminary studies for multi-ton noble liquid detectors [31]. But that choice maximizes PMT-induced backgrounds and costs; such designs allocate a large fraction of target to shielding, and scale dreadfully. System complexity, such as in a practical realization of the drift electric field is maximally aggravated. Two other possibilities seem far better.

First, coating the entire surface of the detector with tetraphenylbutadiene (TPB) converts UV to blue $(420 \mathrm{~nm})$ with high efficiency [32]. The blue light then has a chance to bounce around until it strikes a PMT. In pure gas-phase xenon $(173 \mathrm{~nm})$, this has been shown to improve optical efficiency relative to Teflon by a factor of three to four [33]. As some absorption occurs at each bounce, this choice still requires an unpleasantly large number of PMTs in the cathode plane region to 
detect a reasonable fraction of the converted light. At most, maybe $2-5 \%$ of the original VUV light is detected.

This solution also imposes a significant loss of fiducial volume because a "back region" must be dedicated solely to grade the cathode potential back to ground so PMTs can be operated safely near the cathode. This back region is operated at the highest practical field to minimize lost volume. But the back region can cause real trouble since light can be generated within it, but no ionization information is recovered; thus this reverse field region can generate unwanted events with highly unbalanced $\mathbf{R} / \mathbf{I}$.

A far better way seems possible. As noted above, the conversion efficiencies of two commercial wavelength-shifting (WLS) plastics [34] display maximal conversion efficiency at $\sim 290-310 \mathrm{~nm}$ (see figure 5), perfectly overlapping the emission bands of TMA. This is a truly wonderful circumstance.
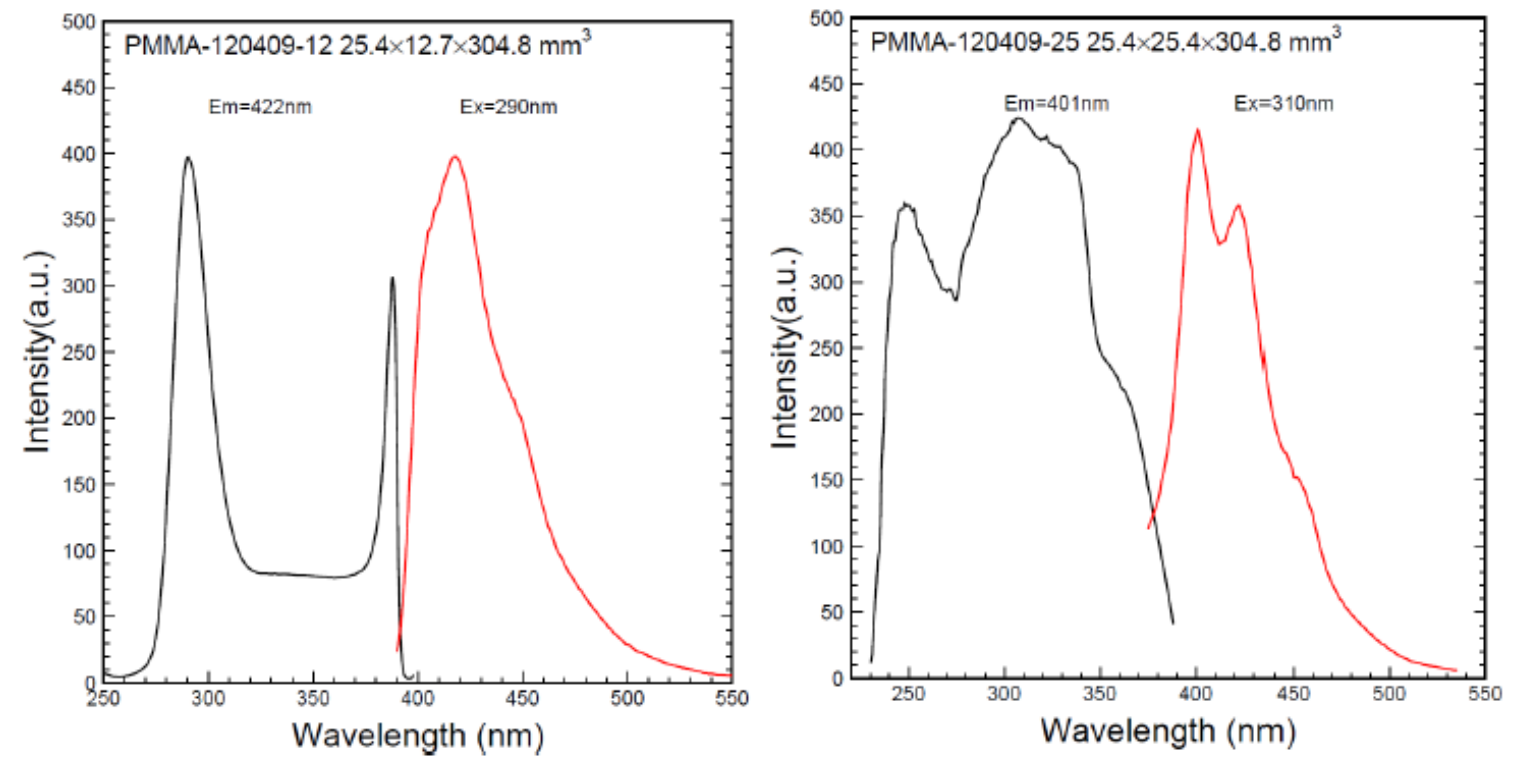

Figure 5. Excitation and emission spectra are shown for two samples of WLS products from ELJEN [34]. The peak excitation efficiency matches perfectly the emissions of TEA and TMA. However, normalizations of the spectra are arbitrary, so optical conversion efficiencies cannot be deduced from the data.

In gas phase xenon, WLS bars can trap more than $50 \%$ of internally converted (blue) light. Instead of converting light at an exterior surface with TPB and having the light bounce until it hits a PMT or is absorbed uselessly, it should be far better to cover the entire interior TPC surface in WLS plastic. The WLS bars can transport the blue light efficiently to a relatively small, and appropriate, number of PMTs. It is desirable to minimize the number of PMTs not only to reduce radioactivity backgrounds, but also to reduce "dark noise". The optical path also allows the slightly radioactive PMTs to be situated behind a shield, likely copper. The drift field can be realized by a combination of a "coarse field cage" beyond the WLS bars i.e., 
behind, at slightly larger radius), with a diaphanous "fine field cage of thin wires in front of the WLS bars.

The famous (but very different) tetrakis(dimethylamino)ethylene (TMAE) [35] may hold some positive surprises with xenon, but is not considered here since its visibly blue-green emission (480 nm peak) will not excite the WLS bars. The path to high optical detection efficiency would be eliminated with TMAE, unless WLS conversion to still longer but useful wavelengths can be realized with high efficiency, and PMTs with high quantum efficiency exist for those longer wavelengths.

If the WLS conversion were $60 \%$, internal capture were as high as about $50 \%$, and PMT quantum efficiency (QE) were $35 \%$ - admittedly optimistic, we could expect $\sim 10 \%$ overall detection efficiency for the $\mathbf{R}$ signal. This performance would be unprecedented in very large detectors with only a few dozen PMTs. Even the tracking plane of a large EL TPC could be covered in WLS plastic, since the untrapped portion of the EL signal that emerges from the WLS backside provides adequate tracking signals. The internally trapped portion of the light born in the tracking plane WLS plane can be extracted at its periphery. The cathode could, for example, be covered in pizza-style WLS wedges, bringing light to WLS staves along the side. A right angle transition using a mirrored surface at $45^{\circ}$ preserves most of the light. No back region is necessary, eliminating an undesirable and dangerous region of the detector. This beneficial simplification improves target efficiency as well. Symmetric TPC geometries also come to mind naturally and may offer a way to reduce systematic effects for any "head-tail" sensitivity (see below).

In any case, detection of the optical signal $\mathbf{R}$, even with $\sim 10 \%$ efficiency, is the quantum bottleneck; in contrast, the ionization signal I can be detected with nearly $100 \%$ efficiency. For this signal, EL can provide gain with negligible noise.

To summarize, the scenario is to maximize columnar recombination, ensure that recombination leads efficiently to UV in the $300 \mathrm{~nm}$ range, and measure it efficiently with $4 \pi$ optical detection: Use either TEA or TMA in the xenon, with WLS plastic bars and plates everywhere, with PMTs likely hidden behind copper.

\section{Detection of the lonization signal I: Electroluminescence}

To realize best possible statistical precision, a substantial investment in the WLS system must be made to detect the fluorescent UV Penning signal $\mathbf{R}$ with the highest practical efficiency. Considering this investment to optical efficiency, it is natural to detect the ionization signal I optically, with UV generated by EL. After the "primary" $\mathbf{R}$ signal is detected, which also establishes the TPC start signal, the cluster of ionization drifts to the EL region. The separation in time makes identification of $\mathbf{R}$ and I signals easy, even though they are both in the form of UV. EL gains, defined as the ratio of UV photons produced per electron entering the EL region, can easily reach several hundred and even beyond 1500 . So I signals are much larger than $\mathbf{R}$, even though the $\mathbf{R}$ population may be larger than I. Dynamic range issues are mitigated to a significant extent because diffusion during drift spreads out the signal 
in time, as does the finite width of an EL gap. In any case, the dynamic range requirement is defined by the huge, but highly extended, signals from $\beta \beta$ events.

With EL and complete WLS coverage, a relatively small PMT ensemble can detect more than 10 or 20 photoelectrons per primary electron entering the EL region, so it is straightforward to detect a single drifting electron. In principle, the threshold for WIMP detection would be limited by PMT noise and UV optical conversion/detection efficiency for the R signal; in practice the detection threshold for tracking - robustly locating the event at the tracking plane - may be the limiting factor. A tracking plane based on SiPMs (called MPPC by Hamamatsu) may provide this capability naturally, but individual sensor area may need to be $\sim 10 \mathrm{~mm}^{2}$.

\section{Other Important Considerations}

\section{Head-tail effect?}

If a strong head-tail effect is present in a direct dark matter search technique, fewer events are needed to establish a robust presence of directionality; in ideal circumstances, perhaps as few as O(10) may suffice [36]. Since the unorthodox columnar recombination scenario presented here compares the angle of two vectors - the drift field and the recoil direction, it is not a priori obvious that a strong headtail effect should be present in the columnar recombination signal. If it does exist, it must depend on whether the nuclear recoil velocity is parallel or anti-parallel to the drift field. Neglecting ionization density variations and velocity effects, the "average" primary electron experiences $\sim 50 \%$ of the ions, independent of parallel or antiparallel orientations of track and field, so a head-tail effect is not obviously present.

Nevertheless, it is interesting to consider how such an effect might be present, which conditions might optimize a head-tail effect, and what sort of effort would be needed to determine a head-tail sensitivity. In consonance with other works, the "head" is defined as point where the track is finished, and "tail" as the starting point.

For these low-energy nuclear recoils, the ionization and excitation densities are highest at the beginning, or "tail", and least at the "head" as the ion stops. The probability of recombination is plausibly greater where ionization density is higher - at the tail - because the collective electrostatic effect of the essentially immobile "tail" ions is larger. The collective electrostatic effect of the ions tends to focus or retain the ephemeral primary ionization in closer proximity than would be the case in the weaker "head" ionization density.

If the recoiling nucleus is moving parallel to the drift electric field, the electrons move in the opposite direction. Some "tail" electrons may leave the track before the event is complete, but are still created in a high ionization density region. Electrons from the "head" traverse the entire ion column. On the other hand, when the nuclear recoil is anti-parallel to the electric field, electrons move in same sense as the nuclear recoil. If the electron drift velocity is less than the nuclear recoil velocity, 
as expected, electrons follow behind the recoiling nucleus. Electrons from the "tail" region will move past most ions in the tail, offering the maximum chance to encounter an ion, whereas, at the head, electrons experience a relatively small ionization density. Thermalization processes also enter prior to recombination, complicating speculation. Overall, it does not seem easy to assert with confidence that a strong head-tail effect will exist due to the ionization density differences.

Curiously, if the circumstance were that the electron drift velocity is larger than the nuclear recoil velocity, primary electrons would outrun the developing ionization front. They would see essentially no primary ions and recombination would be negligible. In this picture, a head-tail effect is maximized for the primary ions. If one allows, for discussion, a very high electron drift velocity, such as $v=100 \mathrm{~mm} / \mu \mathrm{s}$, then the electrons would indeed outrun a xenon nuclear recoil of about $7.5 \mathrm{keV}$. In this hypothetical case, no recombination of primary electrons would be expected. A head-tail effect would be maximal, since in the opposite track-field orientation, recombination would occur. For a drift velocity of $100 \mathrm{~mm} / \mu \mathrm{s}$, electron temperatures must necessarily lie well above thermal; even for $1 \mathrm{ev}$, electron speeds would be around six times thermal, or $600 \mathrm{~mm} / \mu \mathrm{s}$.

Contrary to intuition, then, a head-tail effect might be most pronounced for the low energy recoils! Given that most WIMP-induced events (if any at all) lie in the lowest energy region, it is worth keeping this possibility in mind. This path of inquiry could turn out to be highly fruitful, although it appears now as a curiosity. To summarize, a symmetric TPC aimed at Cygnus might observe a very large sidereal head-tail effect in $\mathbf{R} / \mathbf{I}$ for nuclear recoils below some energy threshold, and very little for nuclear recoils at higher energies! This would be a rather robust signature for directionality in dark matter, but is likely fanciful. A drift velocity in the range of $100 \mathrm{~mm} / \mu \mathrm{s}$ in any xenon mixture appears unrealistic. A high drift velocity also requires a high drift field, and causes energy resolution degradation in the search for $0 v \beta \beta$ decay in ${ }^{136} \mathrm{Xe}$ due to energy gained from/lost to the TPC drift field by daughter electrons. Still, the idea seems attractive enough to warrant further study.

\section{Discrimination against $\gamma$-ray backgrounds}

The two most important discriminants for electron/nuclear recoils are the ratio $\mathbf{R} / \mathbf{I}$ and multiple-site topology criteria. For $\mathbf{R} / \mathbf{I}$, intrinsic fluctuations between $\mathbf{I}$ and $\mathbf{R}$, and statistical precision in measurement determine discrimination power.

A special virtue of gas phase xenon is the presence of well-behaved fluctuations in the partition of deposited energy between scintillation and ionization [17]. In LXe, these fluctuations are anomalously large due to enhanced recombination in regions of high ionization density, primarily from $\delta$-rays. In LXe, the Fano factor $F \approx 20$, whereas in gaseous phase xenon below $\rho \leq 0.55 \mathrm{~g} / \mathrm{cm}^{3}$, the measured Fano factor $\mathrm{F}$ $=0.15 \pm 0.03$ [27]. This huge difference has direct impact for both energy resolution measured by the ionization signal alone, as well as $\mathbf{R} / \mathbf{I}$ discrimination, involving 
both ionization and scintillation. Defining $\mathrm{E}$ as deposited energy and $\mathrm{W}_{\mathrm{I}}$ as the average energy loss to create an electron-ion pair, $\delta \mathrm{E} / \mathrm{E}=2.35(\mathrm{FW} / \mathrm{E})^{1 / 2} \mathrm{FWHM}$.

For the familiar $662 \mathrm{keV} \gamma$-ray of ${ }^{137} \mathrm{Cs}$, the intrinsic resolution of the ionization signal is $\delta E / E=0.6 \%$ FWHM. At LBNL, with colleagues at Texas A\&M, we have constructed and operated a small high-pressure pure xenon gas TPC with an EL gain stage [37]. For the ${ }^{137} \mathrm{Cs} 662 \mathrm{keV} \gamma$-ray, $1 \%$ FWHM resolution is obtained with this device; this excellent resolution is illustrated in figure 6. A system based on avalanche gain cannot reach this performance. For the $0-v \beta \beta$ decay of ${ }^{136} \mathrm{Xe} \rightarrow$ ${ }^{136} \mathrm{Ba}+2 \mathrm{e}$, the energy is $2457 \mathrm{keV}$ and the intrinsic resolution of the ionization signal is $\delta \mathrm{E} / \mathrm{E}=0.28 \% \mathrm{FWHM}$ (gas phase) [20]. By itself, this theoretical value is an impressive resolution.

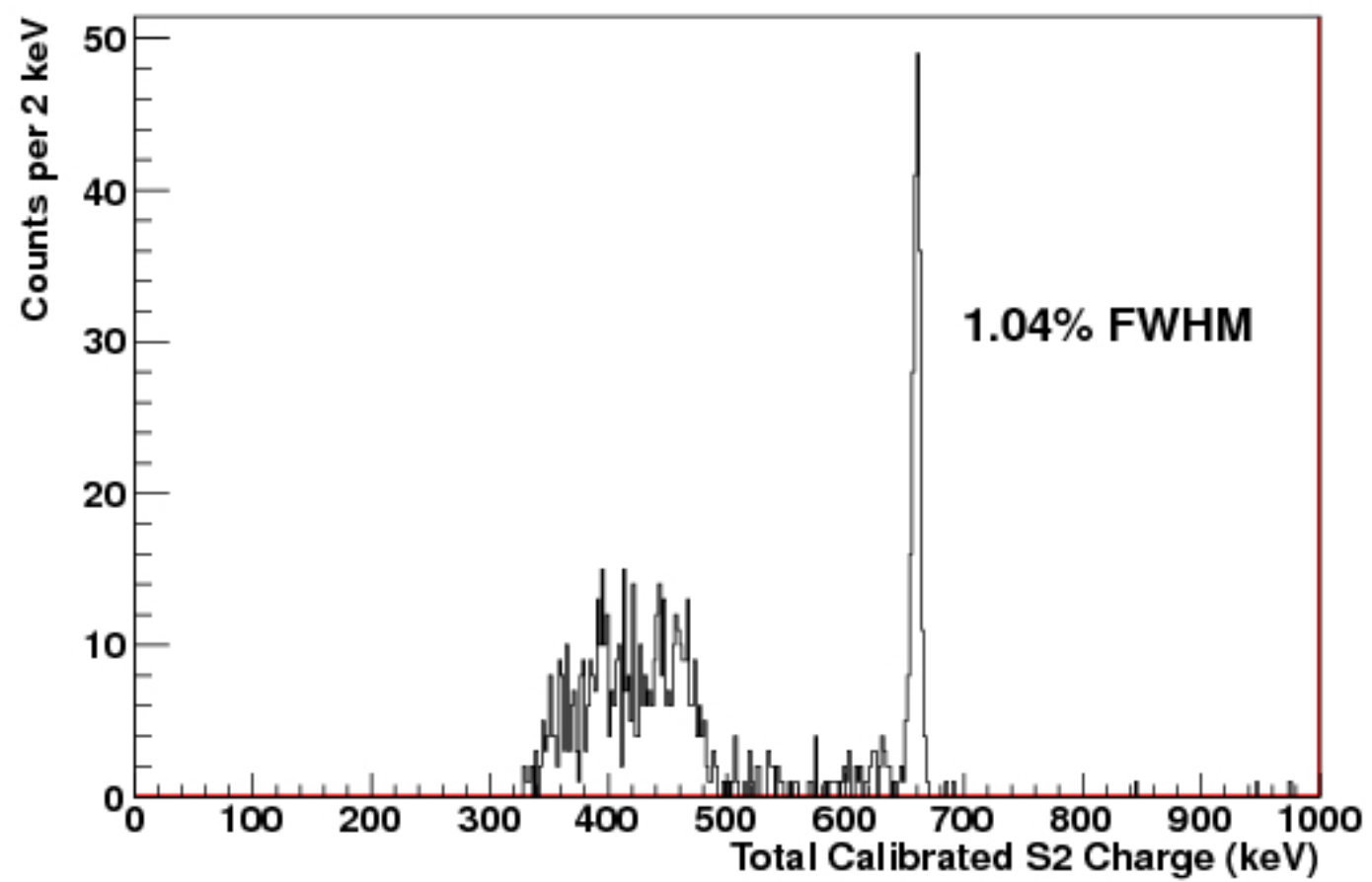

Figure 6. Energy resolution for the ${ }^{137} \mathrm{Cs} 662 \mathrm{keV} \gamma$-ray, as measured in the LBNLTAMU high-pressure pure xenon gas electroluminescent TPC, using only the ionization signal. A small correction has been applied to correct for attenuation during drift by electronegative impurities. The TPC has no explicit tracking, so a strict radial cut has been applied to the fiducial volume, so that no other corrections were necessary. This superb resolution has positive implications for both direct WIMP search and the search for $0-v \beta \beta$ search in ${ }^{136} \mathrm{Xe}$.

Thus, for the ionization signal alone, the Fano factors imply that the gas phase has an intrinsic advantage of about a factor of about 11 relative to LXe. If the scintillation signal is also captured, the impact of partition fluctuations can be reduced. However, statistical precision cannot be completely recovered in practice due to finite geometrical coverage and finite quantum efficiency of the photodetectors. In most systems, the overall efficiency for scintillation detection is a few 
$\%$, rarely reaching or exceeding $10 \%$. As the fraction of energy contained in the LXe optical signal is substantial, the large fluctuations in that signal prevail for LXe. In principle, the HPXe detectors can also improve energy resolution a bit by including the scintillation signal.

The primary scintillation is used here only to provide a trigger for drift time measurement. Amplification by EL is linear and can be essentially noiseless, a unique feature among familiar gain mechanisms. Photo-detectors capture a fraction of the optical signal, returning the information to a charge signal. The noise contribution can be made comparable to or less than the impact of the Fano factor, and is added in quadrature so that energy resolution can be within a factor two of the intrinsic limit.

For a direct WIMP search, the implication of a factor of $\sim 11$ smaller fluctuations between scintillation and ionization is that superb S2/S1 resolution (corresponding inversely to R/I here) may be reasonably expected for the gaseous phase. However, for gaseous xenon with molecular additives such as TMA, the average $\mathbf{R} / \mathbf{I}$ for electron recoils has not been measured. Nor have these quantities been measured for nuclear recoils.

Nevertheless, R/I response versus energy should yield a very narrow band for both recoil types. If the $\mathbf{R} / \mathbf{I}$ bands for electron and nuclear recoils display Gaussian characteristics, as expected, and if separation of the $\mathbf{R} / \mathbf{I}$ bands for electron and nuclear recoils is large relative to width - as expected - the overlap will be small and exponentially damped. In that case, hugely superior discrimination between electron and nuclear recoils relative to LXe may be realized. To illustrate this expectation, a somewhat idealized simulation result is shown in figure 7 for the expected S2/S1 electron recoil signals in pure HPXe, assuming a Fano factor F $=0.15$ and an optical efficiency of $10 \%$.

The narrowness of the S2/S1 band can be compared with the much wider band observed in LXe direct dark matter searches such as in [38]. Since no data are available for nuclear recoils in HPXe, with or without molecular additives, a reasonable comparison is presently not possible, and no simulation is provided.

Nevertheless, as argued below, the ratio of S2/S1 ratios might be in the range of $\sim 50$, suggesting a gap of about 1.7 on $\log _{10}$ scale, and placement of the nuclear recoil band around 2.1 in figure 7 . If even approximately true, the discrimination will be vastly superior to LXe; in practice, the discrimination is more likely to be determined by the extent to which ionization and scintillation signals are collected with well-understood efficiencies from the entire active volume. 


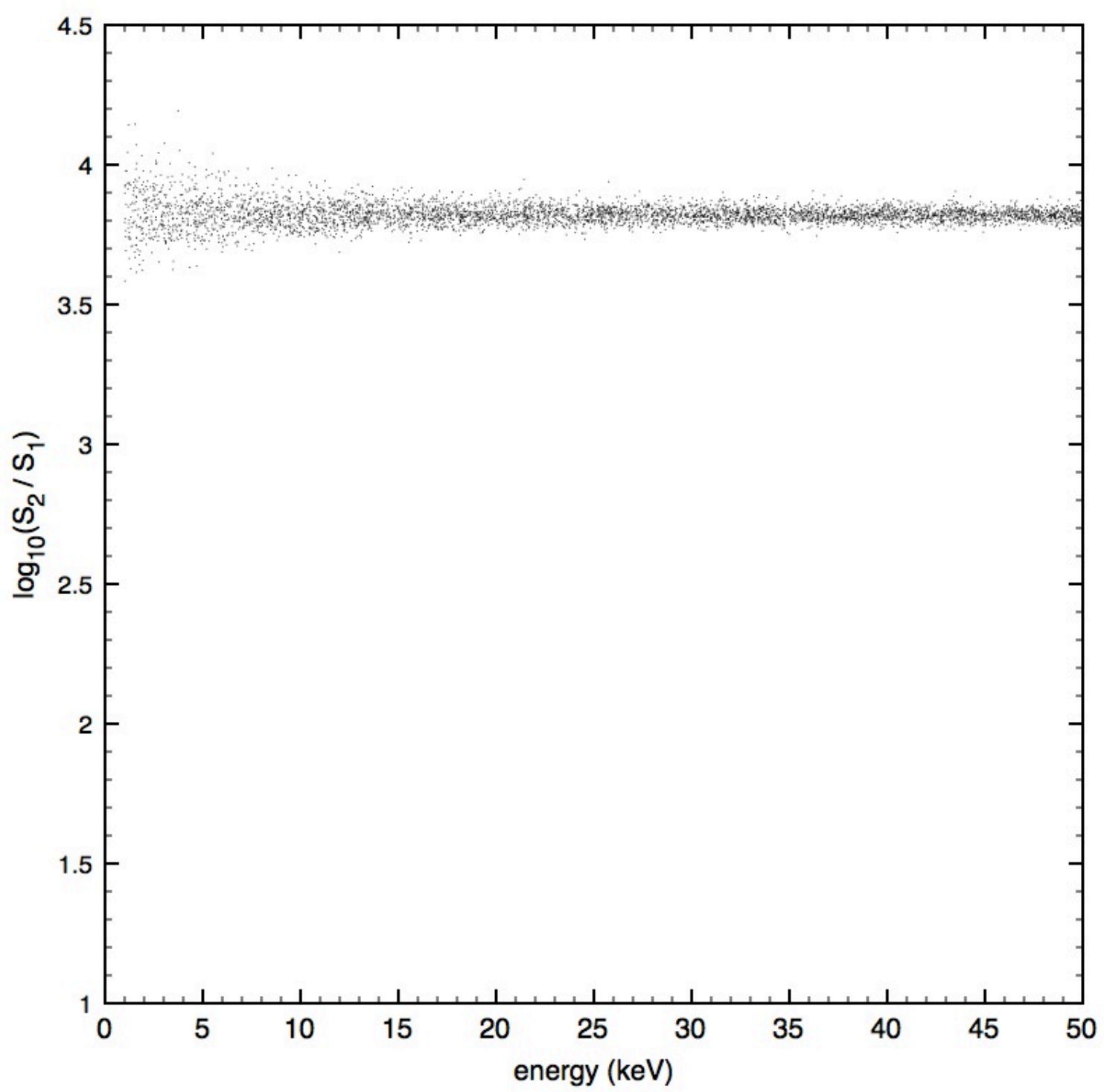

Figure 7. A simulation of the ratio $\mathrm{S} 2 / \mathrm{S} 1$ is shown for electron recoils in a large HPXe detector at 10 bars. The assumptions include a Fano factor $\mathrm{F}=0.15$, and an optical efficiency of $10 \%$. The band placement on the vertical scale represents an arbitrary gain. A rough guess places the nuclear recoil band at $\sim 2.1$, but no measurements are yet available to support relative placement or simulations.

The much lower $\mathrm{dE} / \mathrm{dx}$ for energetic electrons implies that geminate recombination, the Penning process, and charge exchange from TMA to xenon ions will occur as localized two-body events, with rates largely uncorrelated with the average $\mathrm{dE} / \mathrm{dx}$ of the event. Collective effects such as ambipolar diffusion will be 100 times smaller relative to nuclear recoils. The much lower average $\mathrm{dE} / \mathrm{dx}$ of electrons means that the ionization signal I retains most of the original free electron population due to ionization of xenon. The Penning process provides some additional ionization for electron recoil events. As in the nuclear recoil case, 
geminate recombination with xenon ions is returned back to the ionization population by a subsequent Penning interaction.

Furthermore, multiple Coulomb scattering of the electron recoil track is a dominant topological feature, increasingly so at energies below $100 \mathrm{keV}$, so a high level of colinearity of an electron track with drift field will be exceedingly rare. My expectation is that columnar recombination in electron recoils is small, perhaps negligibly small. The presence of $\delta$-rays, prevalent in electron recoils, does not seem likely to compromise discrimination power since the expected operating density, $\rho \approx 0.05$ $\mathrm{g} / \mathrm{cm}^{3}$ is so far away from the fluctuation threshold of $\rho \approx 0.55 \mathrm{~g} / \mathrm{cm}^{3}$, even though the addition of TMA will enhance recombination. As $\delta$-rays are absent in nuclear recoils, their impact can affect electron recoils only. It must be acknowledged, however, that no serious background or discrimination calculation has been attempted yet.

\section{Orient the detector so that the drift field scans WIMP arrival directions}

The angle that the earth's rotation axis makes with the velocity of the galactic plane allows for automatic scanning with regard to putative WIMP flow direction from Cygnus. In figure 8, from [39], the basic concept is illustrated.

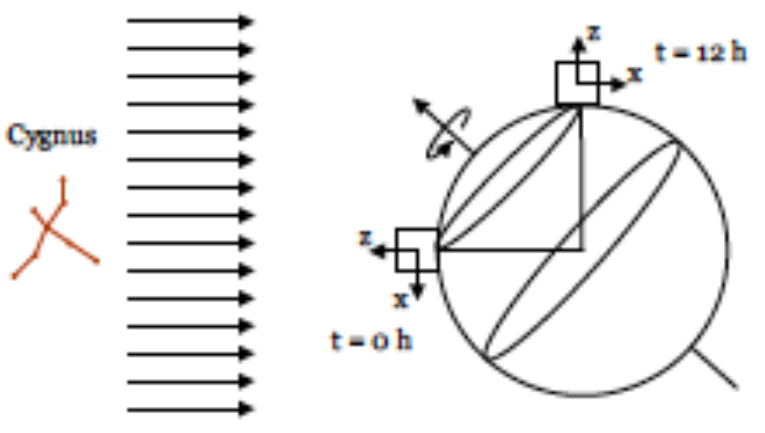

Figure 8. Under the assumption of a WIMP wind favoring the direction from Cygnus, a detector on earth displays varying sidereal response. At any latitude, the detector can be oriented to provide equal sensitivity. (figure is adapted from [39])

The detector would work anywhere, from polar to equatorial regions by orienting to compensate for the angle of the earth's rotation axis. It is necessary to orient the detector such that the drift field is aligned with the expected WIMP flow once per sidereal day. Ideally, the detector should also be anti-aligned once per sidereal day to minimize systematic effects. This is, unfortunately, not possible with a single, fixed, single-ended TPC detector at any latitude. It is possible, however, to construct a symmetric TPC, for which $1 / 2$ has the field aligned parallel once a day, and, simultaneously the the other $1 / 2$ is anti-parallel. Then the modulation difference between the two halves would display the head-tail effect statistically.

It might be better to build two identical asymmetric TPC detectors in opposing alignment, or even three, with one oriented orthogonally to never align with the wind. The WIMP arrival directions are, of course, not aligned perfectly - as 
suggested in figure 8. Local variations in the WIMP flow may be quite different from the simplest scenario, including even a negligible flux at earth, but we can only hope that Nature has not been that unkind to us.

\section{How similar are nuclear recoils to alpha particle tracks?}

While $\mathrm{dE} / \mathrm{x}$ for $\alpha$-particles from the decays of high-Z elements is roughly similar to that of nuclear recoils, viz, about 100 times that of energetic electrons, essential differences exist that complicate direct comparison. These $\alpha$-particles have initial velocities of $\beta \approx 0.05$, which allows them to generate $\delta$-rays as high as $\sim 1 \mathrm{keV}$. The $\delta$-rays and any $\mathrm{x}$-rays leading to ejection of outer-shell electrons form a "penumbra", or shell, of ionization and excitations at a much larger radius than the core track.

As shown in figure 9a, from [40], the core and the penumbra appear to merge for pure xenon, but are visibly distinct for argon and krypton. While this simplified viewpoint suggests that initial tracks from $\alpha$-particles in xenon may be roughly similar to those of nuclear recoils, thermalization of electrons is so much faster in the presence of complex molecules that figure $9 \mathrm{~b}$ does not apply here.

(a)

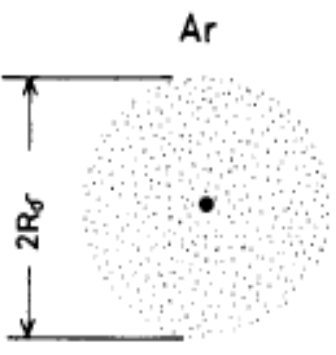

$\mathrm{Kr}$

Xe
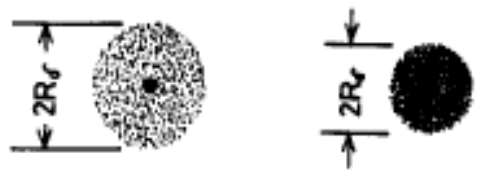

(b)

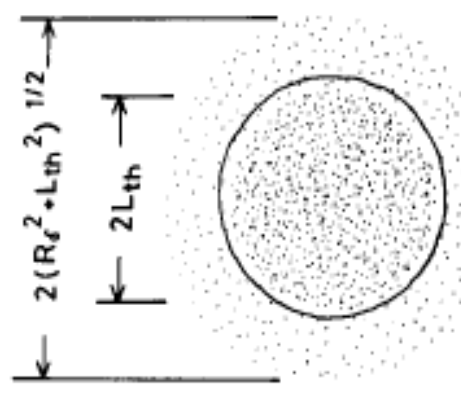

Ar

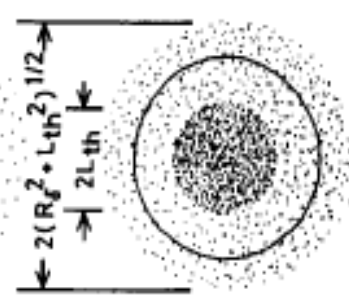

$\mathrm{Kr}$

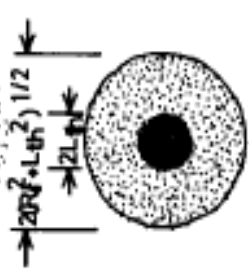

Xe

Figure 9. The time dependence of the cross-sectional view of the track structure relevant to the recombination process in pure noble gases is suggested, from [40]. (a) Initial distribution of "hot electrons; (b) distribution of electrons after thermalization is complete (pure gas only).

Furthermore, since nuclear recoils of interest have initial velocities less than $\beta \approx$ 0.001 they cannot generate $\delta$-rays, in strong contrast to $\alpha$-particles. The primary ionization consists almost entirely of sub-excitation electrons. In the presence of a 
significant fraction of TMA/TEA, sub-excitation electrons will thermalize extremely rapidly. This is the desired circumstance for maximum columnar recombination. The question to be understood is whether this process occurs within the residence time of the electrons while they move past the ion column.

The response of pure xenon to $\alpha$-particles from nuclear decay has been studied extensively [40-43]. This large body of investigation is, however, not directly relevant to the circumstance of nuclear recoils in xenon gas with a significant fraction of complex molecular additive. As noted above, in pure xenon, subexcitation electrons gain/lose energy fractionally on the order of $1 \times 10^{-5}$ in collisions with xenon, so thermalization occurs very slowly. In presence of molecular additives, however, thermalization may occur in a single collision.

Results obtained in the "Gotthard TPC" experiment [44], aimed at the search for 0- $v$ $\beta \beta$ decay in $5.3 \mathrm{~kg}$ of xenon enriched to $68 \%{ }^{136 \mathrm{Xe}}$, are highly relevant. This experiment was operated at 5 bars, with $4 \%$ methane added to provide stable operation of the MWPC gain stage. The presence of methane led to strong "quenching" of the response (observed ionization signal per unit of deposited energy) to $\alpha$-particles - a factor of $\sim 6$ smaller relative to the response to $\mathrm{MeV}$ gamma rays. This quenching of ionization surely must largely be the result of recombination enhanced by methane. The observation of very strong recombination in xenon at 5 bars with $5 \%$ methane strongly supports the concept proposed here, that in xenon at modest densities, with a significant fraction of a complex molecule, electron thermalization is extremely rapid, and hence that strong recombination occurs for $\alpha$-particles; for nuclear recoils with only sub-excitation electrons, recombination should be even more pronounced.

For the same E/N (drift field per unit density) as in the Gotthard experiment, a similar quenching factor of $\sim 5$ for $\alpha$-particles was observed in [45] in xenon at $\sim 25$ bars, a factor of 5 higher total density, but for only $0.6 \%$ methane. ${ }^{4}$ While it is tempting to conclude from this that the controlling factor is absolute density of methane, the similarity could be coincidental.

From these two results, I conclude that a recombination fraction of $90 \%$ (rather than the $\sim 83 \%$ of the Gotthard TPC) is a plausible value for nuclear recoils in xenon gas with $2 \%$ of TMA, likely at densities of $\sim 10$ bars. All in all, it seems safe to conclude that for high values of $\mathrm{dE} / \mathrm{dx}$ - in the range of $\alpha$ particles - and in the presence of a few percent of molecular additives, strong recombination occurs at densities of 5 or more bars. For nuclear recoils, with no penumbra and only sub-

\footnotetext{
${ }^{4}$ Known to quench both ionization and scintillation at low concentrations, methane returns no useful fluorescence signal [46]. With an ionization potential above that of xenon, methane (IP $=12.61 \mathrm{eV}$ ) is inert to xenon ions (IP $=12.14 \mathrm{eV})$ and displays no Penning effect.
} 
excitation electrons, recombination may be reasonably expected to be even more rapid and nearly complete.

\section{How big are the signals $\mathrm{R}$ and I?}

First, consider an electron recoil event of $10 \mathrm{keV}$, in the dangerous energy range, and somewhat above a likely threshold of a few $\mathrm{KeV}$, This event would yield about 400 primary electrons, assuming $25 \mathrm{eV}$ per electron-ion pair. Again assuming electron recoil characteristics, approximately 100 primary excitations would also occur. Assuming 100\% efficiency for Penning transfers, the resultant ionization population is 500. An assumption must be made here for the recombination fraction at the (presently unknown) optimum operating conditions; for discussion, I assume $15 \%$ for electron recoils. After Penning ionization and $15 \%$ recombination, perhaps 425 electrons and $75 \mathrm{UV}$ photons $(300 \mathrm{~nm})$ remain. The intrinsic $\mathbf{R} / \mathbf{I}$ signal is hence $\sim 75 / 425=0.18$.

Consider nuclear recoils with kinetic energy of $30 \mathrm{keV}$, as illustrated in figures $1-3$. From figure 3, the average ionization energy loss is $6.1 \mathrm{keV}$. The implied quenching factor of $\sim 5$ is reasonable, but SRIM does not convert ionization loss directly to free electrons, and therefore uncertainty in interpretation exists. If $\mathrm{w}$ is taken as $25 \mathrm{eV}$, then the average primary ionization is 244 electrons. I assume that for nuclear recoils in xenon gas that primary excitations are more copious than ionization by perhaps some factor like two; this value is quite uncertain, and the factor could be much higher. The excitations are converted by Penning transfer to ionization, yielding a total of 732 electrons. If a recombination fraction of $90 \%$ is assumed, then $\sim 660$ UV photons are produced, leaving only $\sim 75$ free electrons. In this case, the intrinsic R/I signal is $660 / 75=8.8$. The presumed sidereal directionality effect modulates this value.

As the ratio of ratios is about 50, nuclear recoils would presumably fall in a band centered at $\log (\mathrm{S} 2 / \mathrm{S} 1) \approx 2.1$ in figure 7 . Excellent discrimination between electron and nuclear recoils would be available under these assumptions. This should be true even if - as desired - the sidereal modulation is substantial. Since in xenon gas (with a few percent TMA) the anticipated Fano factor for electron recoils is in the range of $0.05<\mathrm{F}<0.15$, primary fluctuations are expected to be very small.

With an assumption of 10\% optical efficiency, $~ 8$ detected UV photons are detected, implying a measurement precision of the $\mathbf{R}$ signal for electron recoils of $\sim 35 \% \mathrm{rms}$. But the $\mathbf{R}$ signal for nuclear recoils would be measured with much better resolution, perhaps $\sim 12 \%$ rms. With an EL TPC, the I signal measurement will not be degraded by gain noise, but rather by the effective "Fano" factors corresponding to the processes that yield the final electron populations in nuclear and electron recoil events. For the nuclear recoil, it seems plausible that the effective Fano factor is large, since three prominent pathways are available - heat, excitation, and ionization. 


\section{How big is the detector?}

As no robust signal for detection of WIMPs has been made to date, it seems inescapable that a detector approaching or likely exceeding the ton-scale is necessary. For example, a symmetric xenon gas TPC with a diameter of $200 \mathrm{~cm}$ and length of $600 \mathrm{~cm}$ holds $\sim 1000 \mathrm{~kg}$ of xenon at 20 bars. This is indeed large, and too large, for example, to fit into the shaft cages at SURF, even in pieces. However, SURF could support a symmetric TPC detector at an intermediate scale of about 400 - 500 $\mathrm{kg}$. So the possibility of a system at SURF holding hundreds of $\mathrm{kg}$ in the active volume seems plausible. Other possible sites offer easier access. A large water tank or water-filled cavity is needed for attenuation of background neutrons and $\gamma$-rays.

\section{What about argon and neon targets?}

Neon, with $A=20$, is an attractive target for a low-mass WIMP search, as the better kinematic match maximizes kinetic energy of nuclear recoils. The scenario described above seems to apply here as well, but with an additional process step. A strong Penning effect exists in neon with a small admixture of xenon; in this case, neon excitations efficiently ionize xenon. However, to maintain the scenario described here, about 2\% of TMA, must also be present. Primary neon and xenon ions would quickly undergo charge exchange with TMA, producing an image of ionized TMA, as before. However, in $\mathrm{Ne}^{*}+\mathrm{TMA}$ collisions, molecular dissociation could be a likely alternative pathway [47]. Therefore, the admixture of xenon should be substantially larger than the $2 \%$ fraction of TMA to ensure that excited neon predominantly interacts with xenon. Perhaps the xenon fraction should be at least $10 \%$, but higher or lower fractions can be used without substantial impact. Thus, some loss of overall efficiency in conversion of detected energy can be expected, but the loss may be less than $20 \%$.

To summarize, in this neon-xenon-TMA scenario, the two-step transfer of energy from the noble gas to TMA is augmented by an intermediate step: excited neon atoms predominantly experience Penning ionization with xenon, while the subdominant population of excited xenon atoms experience Penning ionization with TMA directly. Xenon and neon ions both undergo charge exchange with TMA. Subsequently, the TMA scenario that produces the $\mathbf{R}$ signal unfolds as before. The ratio $\mathbf{R} / \mathbf{I}$ will be somewhat different, but the electron-nuclear recoil discrimination seems likely to be unscathed. A neon-xenon-TMA scenario seems quite viable.

For argon, a Penning effect with TMA or TEA is likely to be minimal, due to a large difference between first excitation energy of argon and the IP of TMA. Known as the "resonant defect" - this difference is large enough that molecular dissociation may predominate. Perhaps a different fluorescent molecule can be identified that offers a strong Penning effect. Until that step occurs, an argon-based scenario is undefined.

\section{Questions abound}

Does the capture of an electron and subsequent de-excitation of TMA behave similarly to photo-absorption at $240-250 \mathrm{~nm}$ (as in [18])? Do electron recoils make $\mathbf{R}$ and I contributions that are substantially different from nuclear recoils, as 
expected? Can electron-nuclear recoil discrimination and directionality sensitivity be optimized simultaneously? Is a head-tail effect observable? How does sensitivity of the $\mathbf{R} / \mathbf{I}$ directionality signal depend on recoil energy? Are conditions optimal for both $0-v \beta \beta$ decay and direct WIMP searches?

Of special concern is that TMA is toxic to humans at a level of parts per thousand, and will raise serious safety issues [48]. However, at a level of parts per million, both TMA and TEA provide to humans a clear olfactory warning signal: rotten fish. TEA is less toxic, but is more corrosive than TMA to some metals, possibly only in the presence of water. Although the most prevalent impurity present in these amines as supplied is water, purification to remove water to sub-ppb levels will be necessary to avoid electronegative attachment of electrons during drift.

\section{Outlook}

Almost all uncertainties involve rates and efficiencies in energy transfer from xenon to TEA/TMA and subsequent conversion of transferred energy to detectable UV. Many of the uncertainties can and will be explored experimentally, while other aspects may also be attacked through simulations. Some of this work can be accomplished with the "TEA-Pot", a new LBNL xenon-amine parallel-plate system, shown as cut-away in figure 10.

Four PMTs (only two shown in this cutaway view, possibly eight if needed) view the gap between the plates. This device allows simultaneous measurement of current and light produced between the two plates, while varying electric field, xenon density, and fraction of molecular additive. The goal of this device is the exploration of concept feasibility, and if feasible, determination of the optimum conditions. The optimum drift field might be low, as noted, to maximize columnar recombination for nuclear recoils.

The first operational phase will utilize $\gamma$-rays of $60 \mathrm{keV}$ from ${ }^{241} \mathrm{Am}$, to gain experience and explore the $\mathbf{R} / \mathbf{I}$ response for energetic electrons for accessible ranges of densities, additive fraction, and drift field. This source will be external, to permit source-in/out measurements. Integrated DC signals for the UV $\mathbf{R}$ and ionization I will be measured by picoammeter. The setup is now in initial operation, with pure xenon, as shown in figure 11.

A second phase with an internal $\alpha$-particle source will approach the domain of nuclear recoils, although it is recognized that the correspondence is not exact. In this phase, signals can be acquired on an event-by-event basis. The source is placed on the cathode. A Frisch grid must be added to allow accurate measurement of pulse amplitude and rise-time - a useful proxy of trajectory angle relative to the drift field.

Measurements of the response to nuclear recoils will involve additional complexity. Directionality signatures obtained from high-Z nuclear decays with back-to-back $\alpha$ particles can measure the dependence of $\mathbf{R} / \mathbf{I}$ on the relative angle between drift field and nuclear recoil directions. For this, new configurations of the LBNL TEA-Pot 
will be necessary to measure the $\alpha$-particle trajectories, perhaps with a CCD replacing the cathode. More likely, completely new devices will be necessary to measure simultaneously and separately, the $\mathbf{R} / \mathbf{I}$ response for nuclear recoils and $\alpha$ particle trajectories. Ultimately, a detector setup involving a neutron beam and recoil neutron detectors is needed to provide an accurate characterization of the performance this concept can deliver.

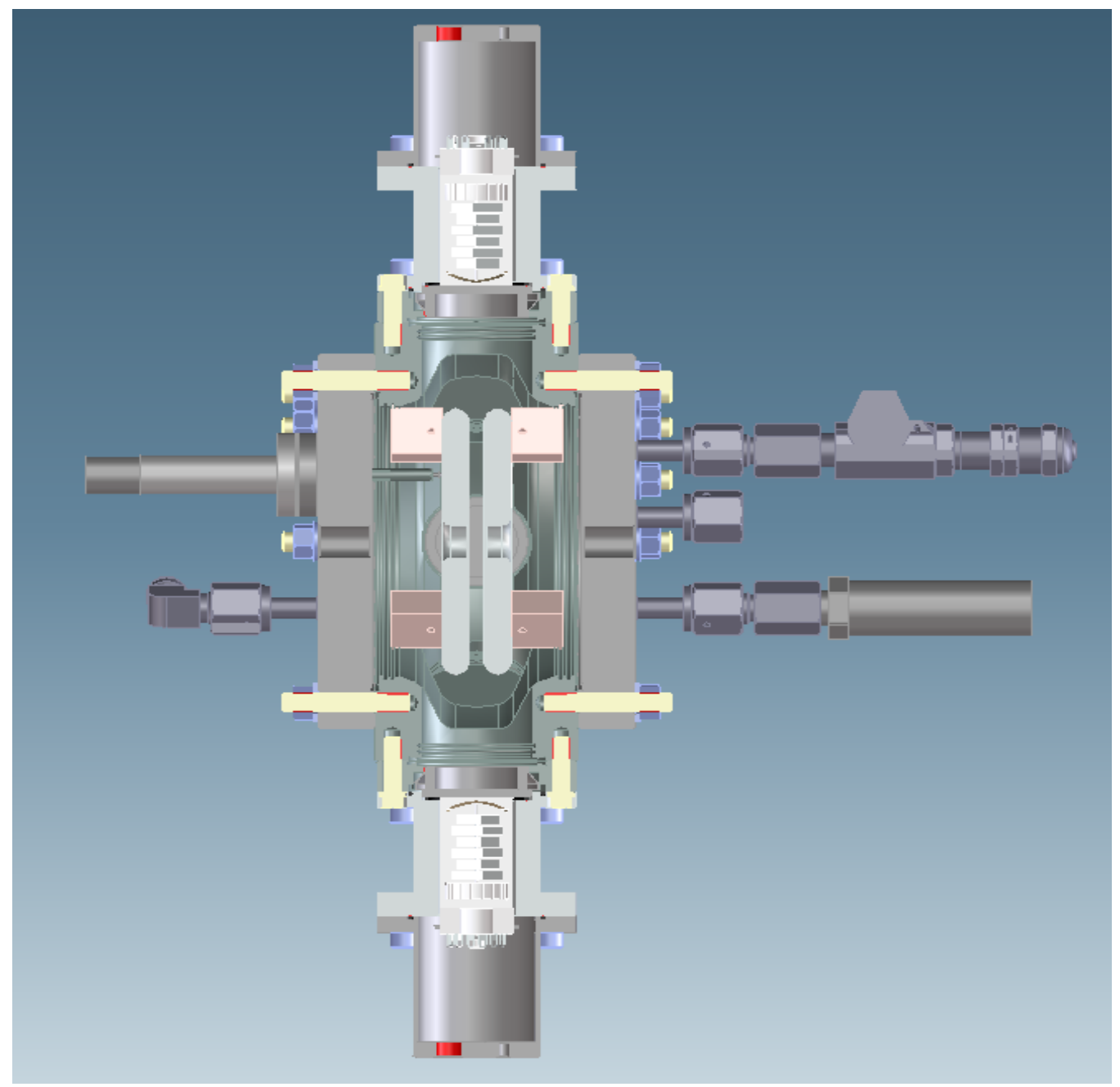

Figure 10. A simple ionization + fluorescence chamber designed at LBNL to allow a full exploration of the response of xenon + TMA or xenon + TEA mixtures over a wide range of densities (up to ten bar), molecular fraction, and electric field. The investigations will also include EL measurements up to and beyond the point of charge gain.

For tracks made by energetic electrons, recombination tends to introduce partition fluctuations between primary ionization and scintillation due to high-ionization regions caused by $\delta$-rays. In pure xenon, these partition fluctuations increase with density and become dominant only above $\rho \approx 0.55 \mathrm{~g} / \mathrm{cm}^{3}$ [17]; this density is a factor of ten or more greater than the operating conditions anticipated here. 
Furthermore, as $\mathrm{dE} / \mathrm{dx}$ is about two orders of magnitude lower for energetic electrons, relative to nuclear recoils, recombination may be largely geminate or quasi-geminate, and ultimately restored to ionization by the Penning effect. Although the presence of molecular additives strongly enhances thermalization of electrons and subsequent recombination, it may turn out that the impact of partition fluctuations caused by $\delta$-rays in energetic electron trajectories is still negligible at the optimum set of conditions. Confirmation will require measurements of electron recoils on an event-by-event basis under these conditions.

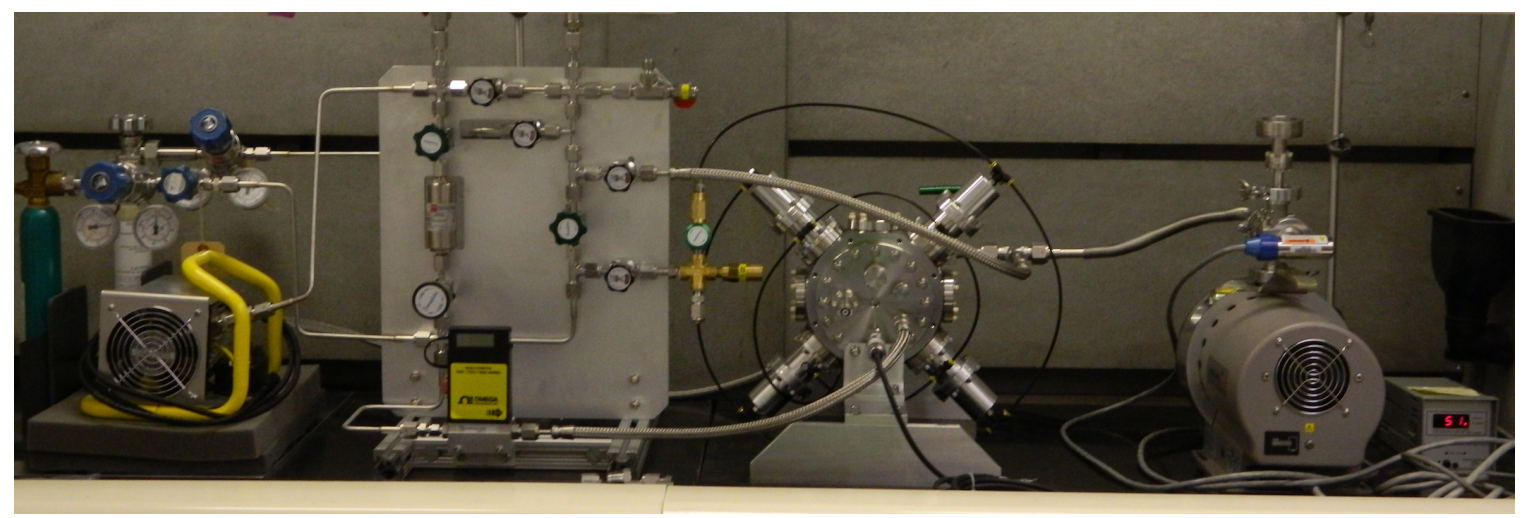

Figure 11. The "TEA-Pot" is shown in a fume hood setup. The two objects on the left are the gas supply, recirculating pump, purifier and valves for control. The TEA-Pot is located at right-center, flanked by a vacuum pump.

\section{Experimental Program for Nuclear Recoils in Xenon Gas}

A program of measurements of electron and nuclear recoil response in pure xenon is being undertaken with the current HPXE TPC at LBNL, which is now in regular and stable operation with an SiPM-based tracking plane. The HPXE TPC may be adaptable to explore the responses of pure xenon and xenon with molecular admixtures to both $\gamma$-rays and neutrons, but not with plastic WLS. The HPXE TPC measurements with pure xenon provide an anchor for understanding more complex behaviors expected with molecular additives. The exploration for optimal operational conditions with amines will be undertaken with the TEA-pot, now in operational status at LBNL. New results in [24] suggest, as suspected, that the optimum admixture of TMA for Penning transfer is near $2 \%$, almost independent of xenon density; this result should reduce the time and effort needed to establish the optimum conditions.

The program of measurements of electron and nuclear recoil response in xenon + TMA/TEA mixtures requires construction and operation of a new small TPC, the OSPREY (Opportunities for Superior Performance in Rare Event Yields). The basic design is shown in figure 12 , below.

The OSPREY detector will have WLS plastic covering the entire interior surface of the active volume, and will be carefully designed and constructed to ensure that any ionizing event within that volume has maximal collection efficiency for both primary ionization and primary scintillation. The cylindrical vessel for OSPREY could be made of a carbon 
fiber composite to admit, with minimal scattering, neutrons and relatively low energy $\gamma$ rays from ${ }^{57} \mathrm{Co}$ or Americium. Our expectation is that the cylinder can be obtained commercially, and we will rely on the LBNL Composites Facility for guidance here. Alternatively, a stainless steel vessel is thin enough that scattering effects are likely still manageable. Neutrons and $\gamma$-rays can enter directly through the cylindrical sidewalls, and both endplates will be equipped with re-entrant tubes to admit neutrons and $\gamma$-rays along the axis. This will facilitate investigations of columnar recombination and headtail effects.

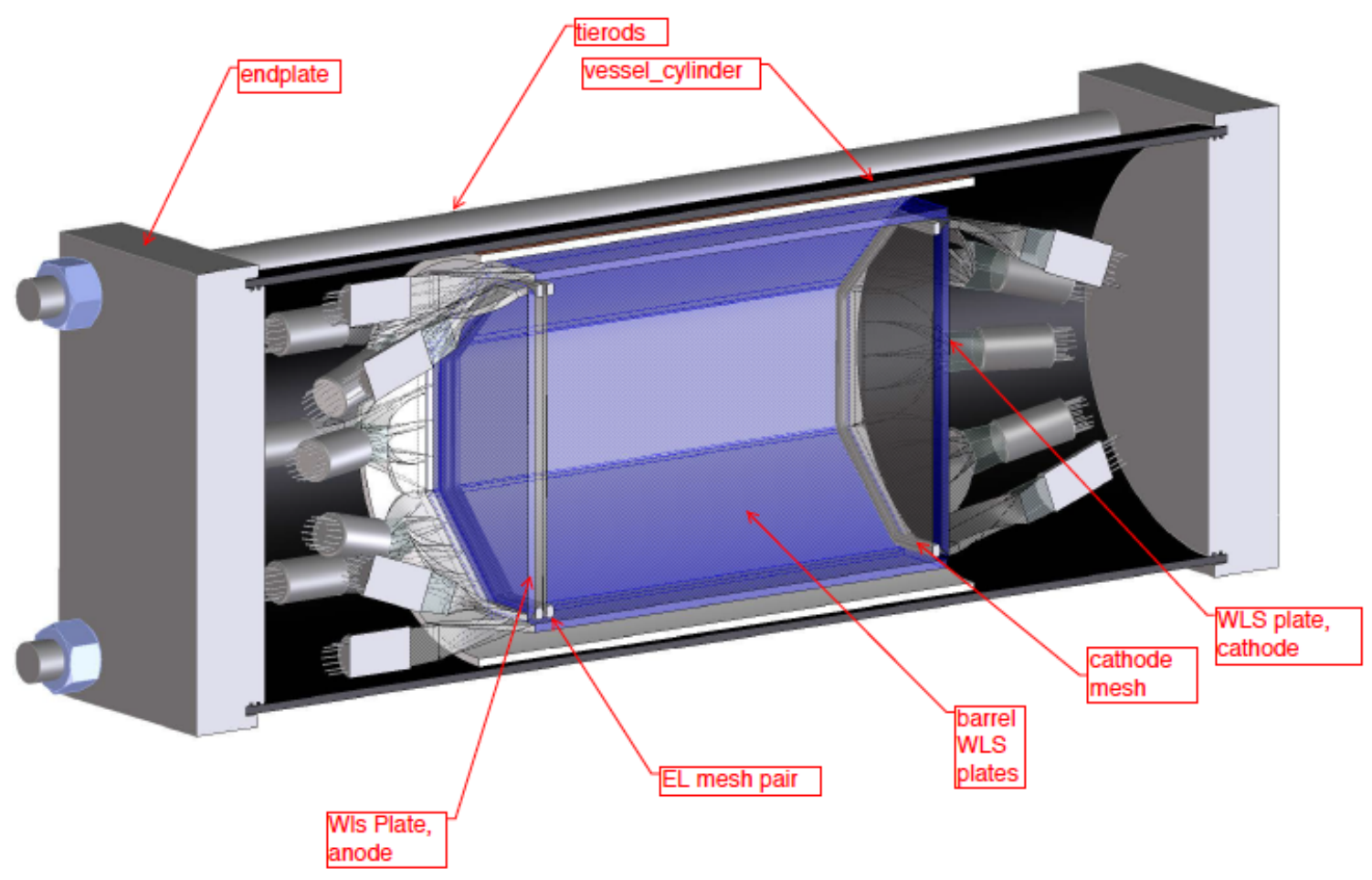

Figure 12. Illustration of the new xenon + TMA/TEA high-pressure TPC "OSPREY" with full WLS coverage. The PMTs are the 1" robust Hamamatsu devices we use and are quite familiar with from our earlier work. Operation at up to 15 bars will be possible. The horizontal axis facilitates transverse and axial entry of neutrons and $\gamma$-rays, by a simple rotation of the stand and neutron detectors. Four high-strength tie rods (only two indicated) support axial stress caused by pressure on the end plates, helping to minimize wall thickness of the cylindrical pressure containment.

Tracking is provided by detection of the untrapped blue light fraction (about 30\%) emerging from the WLS disk just next to the EL mesh pair. An SiPM matrix close to the WLS disk, nearly identical to that constructed for the present EL TPC at LBNL, will enable reconstruction of event topology for electron recoils as well as nuclear recoil localization. Due to refraction as blue light emerges from the WLS plate, only an appropriately small zone in the EL plane will illuminate a given SiPM. No TPB or other coating would be needed on the SiPM surface. The anode mesh will likely be grounded, requiring -10 to $-14 \mathrm{kV}$ for the "gate" mesh, and $-20 \mathrm{kV}$ or so at the cathode plane. The 
light guides at the cathode end will have (non-contact) potential grading to suppress charge creep and discharges along the polished surfaces.

A field cage outside the WLS bars forming the quasi-cylindrical surface will establish the uniform drift field from cathode to mesh gate. Once HV is imposed to create the drift field, radioactive sources will be used to induce mobile charges in the active volume gas. The ions and electrons will follow the initial field lines, distorted slightly by the WLS dielectric constant. Mobile charges in the gas will move to eliminate field lines that emerge or exit from the WLS plastic, leading to uniform extraction of charge from the active volume to the EL plane. The needed charge-up is modest, and once established, charge collection to the EL plane can be close to $100 \%$ throughout the entire volume. Nevertheless, although we cannot expect that ionization produced very near the surface $(<1 \mathrm{~mm})$ can be collected with 100\% efficiency, these events should be detectable as surface events. Based on our success and experience with our current pure xenon system, supported by KA-15, we expect that this TPC can be rapidly designed in detail, fabricated, constructed, and commissioned.

\section{Summary}

A novel approach to directionality sensing of nuclear recoils has been described in outline, with several unproven but quite plausible aspects. The concept exploits columnar recombination in high-pressure xenon gas with an admixture of perhaps 2 $\pm 1 \%$ TMA in a large monolithic TPC. A modest drift field, an electroluminescent gain stage, and wavelength-shifting optical sensing over $4 \pi$ are combined to permit tonscale active masses in a manageable size, still with a very modest number of PMTs.

The important concept of "columnarity" suggests that an optimum xenon density may exist near $\rho \approx 0.05 \mathrm{~g} / \mathrm{cm}^{3}$, about ten bars, or $\sim 1.6 \%$ of the density of liquid xenon, $\rho \approx 3.1 \mathrm{~g} / \mathrm{cm}^{3}$. Sensitivity is lost at LXe density. A strong Penning effect by TMA converts primary xenon excitations (insensitive to nuclear recoil direction) to ionization, increasing the population that can contribute to a columnarity signal, thereby enhancing sensitivity. Providentially, tertiary amines such as TMA also fluoresce with high efficiency in a band (280 - $320 \mathrm{~nm})$ that appears optimally matched to wavelength-shifting plastic.

The flexibility of room-temperature gas detectors to vary conditions such as density, molecular additives, and additive fraction appear essential to realize an overall optimum. An alternative scenario, primarily neon with small fractions of xenon $(\sim 10 \%)$ and TMA ( $2 \%)$, appears viable as well. Argon appears problematic until a fluorescent Penning molecule matched to argon is identified.

This novel concept extracts directional information from within the volume defined by the range of a typical nuclear recoil - on the order of $(2 \mu \mathrm{m})^{3}$. A simple but still interesting figure of merit is the ratio $\mathrm{M}$ of active detector volume to this minimum volume element from which directional information is obtained. For the concept described here, $\mathrm{M}$ exceeds $10^{17}$ for each $\mathrm{m}^{3}$ of active volume; for a monolithic ton- 
scale system, $\mathrm{M}$ is about $10^{18}$. In contrast, for low-density gas TPCs with $\sim 1 \mathrm{~mm}^{3}$ minimum volume and restricted drift length, $\mathrm{M}$ is $\sim 10^{9}$. A more complete figure of merit, of course, should reflect a factor for directional sensitivity - such as the number of events needed for a five- $\sigma$ discovery claim. Cost for competitive sensitivity is also important. Although criteria for discovery, such as "head-tail" recovery, have been explored [49], no consensus has emerged.

The parameters for a WIMP search with optimum directional sensitivity may not be so different than those for a $0-v \beta \beta$ decay search in ${ }^{136} \mathrm{Xe}$; the "optimum" detectors and operating conditions might even be identical [21]. In such a dual-purpose scenario, a switch from enriched xenon (more sensitive to spin-independent WIMP signals and $0-v \beta \beta$ ) to depleted xenon (more sensitive to spin-dependent WIMP signals and "target empty" background run for $0-v \beta \beta$ ) is quite easy. As an easy exchange of active target is impossible for any solid-state system, such as those based on germanium or other crystals, this possibility offers an additional and substantial advantage to a xenon-based concept such as this one.

This concept, based on columnar recombination and atomic/molecular gymnastics, requires substantiation in many respects. With some optimism, a substantial sensitivity advance for nuclear recoil directionality relative to contemporary approaches may be demonstrable with straightforward effort. Should feasibility of this concept for directionality sensing be confirmed, however, a sensitivity advance of about three orders of magnitude is available in a single monolithic ton-scale system relative to approaches that attempt to visualize nuclear recoils.

The plausible costs for ton-scale low-density TPCs systems of comparable sensitivity favor the high-density xenon gas columnar signal approach by a very large factor. Finally, due to the dominant presence of large energy partition fluctuations in LXe, the discrimination against $\gamma$-rays and associated electron recoils may be vastly superior in gas phase xenon, as partition fluctuations in xenon gas at relevant densities are very small.

\section{Acknowledgements}

I thank Victor Gehman, Azriel Goldschmidt, J. J. Gomez-Cadenas, Carlos A. B. Oliveira, and Josh Renner for useful discussions, Carlos A. B. Oliveira for SRIM calculations, and Derek Shuman for engineering consultations. DOE supports this work at LBNL through the KA-15 Advanced Detector Development program. This concept was conceived within the framework of the NEXT Collaboration and project.

\section{References (incomplete)}

1. WIMP hypothesis

2. Hooper et al - fit to DAMA data for low mass WIMP

3. Models of WIMP distribution and flow in our galaxy

4. Velocity distribution - Lewin \& Smith

5. Theories of DM 
6. Analysis of present WIMP situation including DAMA

7. DRIFT

8. DMTPC

9. MIMAC

10. NEWAGE

11. Directional Dark Matter - "D ${ }^{3}$ "

12. Onsager theory and radius

13. Recombination in pure xenon gas

14. SRIM

15. Coherent xenon atomic recoil

16. Conduction band in LXe -delocalization

17. Ramsey \& Bolotnikov

18. Cureton et al

19. Kreuger et al

20. Nygren 2007

21. Nygren 2009

22. Aprile, Bolozhdynya, Bolotnikov, Doke

23. Ramsey and Agrawal

24. Herrera et al

25. NIST Chemistry WebBook

26. DMA IP source

27. Fano factor in pure xenon

28. Resonant charge exchange

29. Sub-excitation electrons

30. Charpak et al

31. Arisaka multi PMT XAX

32. TPB - Vic?

33. NEXT-DEMO

34. ELJEN WLS

35. TMAE

36. DRIFT head-tail argument

37. NEXT-DBDM paper

38. XENON-100 results

39. Cygnus WIMP wind

40. alpha particle core/penumbra

41. alphas in xenon

42. alphas in xenon

43. alphas in xenon

44. Gotthard TPC

45. Pushkin alphas

46. Methane quenching

47. Bauer - photodissociation

48. TMA toxicity

49. Spergel 


\section{Instrumentation Needs for Detection of Ultra-high Energy Neutrinos Peter Gorham, Univ. of Hawaii at Manoa}

Ultra-high energy neutrinos are guaranteed to be present in our universe at a flux level that is determined by known physics but as yet unknown astrophysics. Ultra-high energy cosmic rays have been observed for more than 50 years at energies where they must be already subject to collisions with the cosmic microwave background radiation, a process first elucidated in the 1960's by Greisen, Zatsepin, and Kuzmin. The by-products of such collisions yield the so-called cosmogenic UHE neutrinos, and their flux is a direct measure of the nature and cosmic evolution of the - still unidentified -- highest energy particle hyperaccelerators in our universe. These accelerators are known to achieve energies above $1020 \mathrm{eV}$, seven orders of magnitude above the current LHC.

UHE cosmic rays measure the endpoint of the source evolution function in the current cosmic epoch, but they cannot by themselves determine the sources or their intensity at earlier epochs, because the UHE cosmic rays are absorbed in transit, leaving only the daughter neutrinos to trace out the sources at earlier times. In fact, for all other high-energy astrophysical sources, the current epoch tends to be relatively benign compared to the higher redshift universe, and we have no reason to doubt that a similar behavior will obtain for the UHE cosmic ray sources. Thus UHE neutrino observations are the only feasible way to directly probe the highest energy universe at epochs where it is plausibly at its most intense. Thus detection of UHE neutrinos is a goal of the first rank in high energy astrophysics, and the problem of identifying and characterizing the UHE cosmic ray sources remains one of the most prominent outstanding mysteries in all of astrophysics.

Because of the very low fluxes and interaction cross sections of UHE cosmogenic neutrinos, the natural scale for an observatory-class detector has been coming into definition. While earlier estimates indicated that cubic-km scales could possible reach these fluxes, it is now evident that much larger scales are necessary, with acceptances approaching a Teraton-steradian, or a thousand cubic km of ice observing half the sky. Optical technologies cannot scale from current detectors such as IceCube, which is optimized for lower energies and higher fluxes. In the last decade, a new approach, utilizing the Askaryan effect: coherent Cherenkov radio emission from particle cascades, has emerged as the methodology of choice for these UHE energy ranges. In addition, deep polar ice, while an excellent medium for optical detection of Cherenkov light with $\sim 100$ meter attenuation lengths, and tens of meter scattering lengths, is almost unbelievable clear for radio propagation: with more than a $\mathrm{km}$ attenuation length, and negligible scattering.

A wide range of efforts focused on detection of highly polarized, broadband, impulsive radio events the hallmarks of the Askaryan effect - have engendered a series of experiments, primarily in Antarctica, to realize the goal of cosmogenic neutrino detection. These include the ongoing NASA long-duration balloon experiment ANITA, which observes $\sim 1 \mathrm{M}$ cubic km of ice during several weeks' duration stratospheric balloon flights, and future efforts such as the ExaVolt Antenna (EVA) a large-scale follow-on to ANITA, the Askaryan Radio Array (ARA) which seeks a several hundred square km embedded radio array at the South Pole, and ARIANNA, a similar effort to place radio instruments over a large area on the Ross Ice Shelf.

This goal is not just an astrophysics goal. Once determined, the flux of UHE neutrinos will be stable and predictable to a high degree of precision as models become refined and perfected, in some ways analogous what has happened with the cosmic microwave background mapping. With a well-determined neutrino flux, the possibilities for doing weak interaction physics at 10-100 TeV CM energies on hadronic targets begin to open up, along with flavor physics, and other studies at energies that will not be achievable on Earth in the foreseeable future. Model-independent cross-section measurements are relatively straightforward once the detector scales are large enough, by just considering ratios of event rates from above and below the horizon.

Instrumentation needs in this field have been aided by the rapid worldwide development of wireless $\mathrm{RF}$ technology, but now that wireless is moving to multi-GHz microwave bands, the development burden of improved low-noise amplifiers, exotic types of radio antennas, and ultra-sensitive coherent triggering systems is falling back on the investigators. Low-power, ultra-fast sampling RF waveform digitizers are a particular need that requires specific sponsor support, because it has only limited commercial utility. And finally, a continuing energetic support of polar science programs, and the infrastructure necessary to support them, is essential to this field. The most remarkable and precious dielectric on Earth - at least for purposes of UHE neutrino detection - is Antarctic ice, a threatened and limited resource. 


\title{
Low Background Materials for Direct Detection of Dark Matter
}

\author{
Jeter Hall for the PNNL low background materials and assay team \\ Pacific Northwest National Laboratory
}

(Dated: April 16, 2013)

\begin{abstract}
The direct detection of Weakly Interacting Massive Particle dark matter interacting with terrestrial detectors is an active field in the Cosmic Frontier. The basic principle for these direct detection experiments is to remove all known types of radiation and look for this fundamentally new radiation in the laboratory. These experiments require state of the art assay of experimental construction materials for radioactive contamination and may require custom materials production. Additionally, sensors and electronics near the active detector volume can easily dominate the radiation budget of dark matter experiments, so reducing the radioactive contamination in these devices would reduce the risk and improve the scientific output of dark matter searches.
\end{abstract}

\section{PHYSICS MOTIVATION}

A wealth of cosmological observations have been made over the past few centuries. These are well described by the Standard Model of Cosmology where the large scale structure of the Universe has been created by the collapse of dark matter structures frozen shortly after the Big Bang [? ]. The only detections of this dark matter component are through gravitation measurements of galactic orbits, galaxy orbits, and large scale structure. The physical nature of this dark matter component is still mysterious. In addition to dark, this component of the Universe must be cold (or at most tepid) and non-baryonic. Weakly Interacting Massive Particles (WIMPs) are often a consequence of symmetries of nature beyond the Standard Model of Particle Physics and could be a significant component of the dark matter [? ]. If WIMPs are indeed a significant component of the dark matter, then they could exist with sufficient density and baryonic interaction cross-section, that they could be detected by laboratory experiments [? ].

There has been considerable progress in sensitivity towards interesting WIMP candidates over the past two decades. Although there are other promising techniques for dark matter searches in the presence of backgrounds, annual modulation and directional detection, the best sensitivities have been achieved with experiments that try to suppress the backgrounds to negligible levels. WIMP search detectors were quickly dominated by cosmic radiation at shallow sub-surface laboratories [? ]. After moving to deep underground laboratories to shield from the cosmic backgrounds, the radioactive contamination in and near the active detector volumes has provided the dominant background and has limited the physics reach of all searches thus far. Controlling radioactive backgrounds is one of the primary goals for next generation dark matter searches. The most sensitive search thus far was published by the XENON100 Collaboration with a 225 (live) day exposure of $35 \mathrm{~kg}$ (fiducial volume) of liquid xenon with only two events observed [? ].

The general approach to radioactive backgrounds is to reduce, shield, and finally discriminate against interactions with known types of ionizing radiation. The menagerie of direct detection experiments is a tribute to the clever approaches that have been developed to discriminate against electronic recoils from gamma and beta backgrounds. Typically, neutron backgrounds are the most insidious because they induce nuclear recoils identical to the expected WIMP signals and self-shielding agains neutrons typically cuts a prohibitive amount of the active volume. Many of these material requirements are similar to other rare event searches in these energy ranges, for example low energy neutrino and $0 \nu \beta \beta$-decay experiments. However, the emphasis on neutron backgrounds is different than the emphasis on, say, $2 \mathrm{MeV}$ gamma rays in germanium based $0 \nu \beta \beta$-decay experiments.

\section{MATERIALS}

Since neutrons backgrounds almost always limit an experiment, there is a special emphasis on removing uranium and thorium contamination since they can produce neutrons through spontaneous fission or $(\alpha, \mathrm{n})$ depending on the matrix in which they are embedded. The amount of uranium and thorium contamination allowed in particular materials varies due to the location and matrix, but taking spontaneous fission as an inevitable process, a contamination of $1 \mathrm{mg}$ of ${ }^{238} \mathrm{U}$ results in a production of 1.2 neutrons per day. In context, the next generation dark matter experiments have background goals approaching $<1$ event year $^{-1}$ in detectors with fiducial masses of $\sim 1$ ton.

Although there are a variety of dark matter experiments, a convenient dissection of the experiments is that they are made of shielding materials, detector materials, and electronics.

\section{A. Shielding}

Traditionally, small detectors have used a mixture of lead to shield external gamma rays, and hydrogenated material, such as polyethylene, to shield external neutrons. As dark matter detectors have grown in size, more 
and more experiments are moving to water based shielding. Large ( $\sim 3$ meter thick) shields are common as these are thick enough to serve as both gamma and neutron shielding.

\section{B. Detector Materials}

All dark matter detectors consist of some active element, germanium and xenon as examples, which are instrumented and contained in some sort of vessel. Some experiments require pressure vessels and others require vacuum vessels for containment. These vessels must satisfy leak and mechanical constraints. Materials currently used for containment include stainless steel, copper, and titanium. Generally, the radioactivity from these materials must be less than $\sim 100 \mu \mathrm{Bq} \mathrm{kg}{ }^{-1}$ to meet the specifications of dark matter experiments.

\section{Electronics}

The sensitive volumes are instrumented with various sensors and electronics. Many of the scintillator based detectors utilize photomultiplier tubes (PMTs) and developing low radioactivity light detectors has been an active area of research. However, PMTs are often the dominant source of both neutron and gamma backgrounds in experiments based on the noble elements. Even with their high backgrounds, PMTs offer the low noise, high sensitivity, and large areas required by dark matter experiments, so they are the photodetector used in almost all scintillator based experiments. Other lower background devices such as large area avalanche photodiodes have been successfully deployed in other rare event searches [? ], but the requirement for single photoelectron sensitivity currently precludes their use in dark matter detectors. Silicon photomultipliers are an attractive option, but their small areas and high dark rates are prohibitive.

Other, more basic, electronics components used in almost all dark matter experiments are potential sources of unwanted backgrounds including cables, circuit board substrates, resistors, and capacitors. Devices made from ceramics or with significant glass content typically contain significant uranium, thorium, and potassium contamination. A large amount of effort is spent by each collaboration to screen potential components and substrates to find sufficiently low background pieces. Often the radioactivity requirements are coupled with other unique requirements such as thermal conductivity.

\section{Surface Contaminations}

It is not sufficient to screen materials for bulk contamination of radioactivity. Surface contamination, often from chemical deposition or radon plate out, is an important consideration for many rare event searches.
This is especially true for surfaces that face the active detector material. Typical surface contaminants are due to chemical deposition and radon plate out, so these contaminants include isotopes that $\alpha$-decay inducing worries about $(\alpha, \mathrm{n})$ neutron backgrounds from surfaces that do not have line of sight to the sensitive regions of detectors. The accumulation of radon daughters during material storage and handling is an important consideration for dark matter detector construction. These backgrounds can be difficult to assay at levels important for modern dark matter experiments since direct alpha, or low energy beta, screening is the most sensitive to radon daughters such as ${ }^{210} \mathrm{~Pb}$.

\section{ASSAY}

Assay of materials for radioactive isotopes is required to reduce risk and improve the scientific output of each dark matter experiment. Simulations of specific detector geometries is required to give contamination budgets for specific components. Then each candidate material is assayed to qualify a material or commercial product for a specific detector component. The most common methods of assay are through gamma counting with high purity germanium detectors (HPGe), mass spectroscopy, and neutron activation analysis (NAA). The requirements on sensitivity, sample size, and isotopic analysis will drive the decision on assay technique. High sensitivity HPGe analysis requires large samples, up to a few kilograms of material, whereas mass spectroscopy and NAA can achieve important radioactive contamination levels with $\sim 1$ g samples.

HPGe assay is a useful tool for material assay. The ${ }^{238} \mathrm{U}$ and ${ }^{232} \mathrm{Th}$ decay chains emit characteristic gamma rays, however the decay chains can be broken either through chemical means or at the radon break points, so gamma spectroscopy requires care at the stage of interpretation. Pure gamma emitting isotopes such as ${ }^{40} \mathrm{~K}$ are ideal candidates for HPGe assay.

Mass spectroscopy is a useful tool because it will measure exact isotopes of interest and extrapolations through decay chains is not required. Additionally, penetrating gamma rays are not required for elemental identification. Glow discharge mass spectroscopy (GDMS) is a useful tool because a variety of isotopes can be measured at the same time and sample preparation is minimal. However, GDMS has poor sensitivity, so is typically only useful for prescreening materials for more sensitive assay techniques. Inductively coupled plasma mass spectroscopy (ICPMS) is more sensitive to isotopes of interest and is a important tool for modern dark matter experimentalists. The most sensitive ICPMS requires dissolving samples for concentration, and special techniques are required to perform the most sensitive ICPMS on the large variety of materials required for dark matter detectors. ICPMS does suffer from important interferences that reduce the sensitivity for certain isotopes, for example ${ }^{40} \mathrm{~K}$ is diffi- 
cult due to the argon gas used in ICPMS.

NAA is sensitive to a number of isotopes of interest for the dark matter community. Again this technique can probe specific isotopes of interest, such as ${ }^{238} \mathrm{U}$, without relying on decay chain assumptions. NAA requires careful sample preparation and specialized analysis. NAA, especially when combined with radiochemical separation, can result in some of the best minimum detection levels currently possible.

\section{CUSTOM MATERIALS}

It has been shown that modern metallurgical techniques and special attention to polymerization precursors can reduce the radioactive contamination in materials. Collaborations interested in rare event searches have shown that custom materials can be developed when commercial materials do not meet specifications in terms of radioactivity.

For example, high- $\mathrm{Z}$ construction materials can be purified using electrochemical techniques to levels resulting in $<1 \mu \mathrm{Bq} / \mathrm{kg}{ }^{238} \mathrm{U}$ and ${ }^{232} \mathrm{Th}$ activities. Not only can the radioactivity be reduced in these materials, but the mechanical properties can be controlled better than in typical high-throughput industrial techniques. This has been demonstrated for copper by the Majorana Collaboration, and could also be accomplished for other important metals such as gold, lead, nickel, tungsten and iridium as well as some metal alloys such as a number of copper alloys.

Custom low background electronics components such as capacitors, resistors, and cabling could be produced in sufficient quantities that the up-front development costs could be shared economically across future experiments reducing the need to constantly assay large numbers of these components.

\section{SUMMARY}

Low background materials are important for dark matter research and their importance will only grow over the next decades. Insufficient care with seemingly insignificant detector components can drastically reduce the scientific impact of experiments [? ], however sufficient care can produce experimental backgrounds within desired specifications [? ]. The ability to source, assay, and keep materials free of radioactive contamination is crucial to reducing risk and improving scientific output from the efforts to directly detect WIMP dark matter interactions with terrestrial detectors. 


\title{
Physics Motivation for WIMP Dark Matter Directional Detection
}

\author{
David Nygren \\ Lawrence Berkeley National Laboratory
}

A robust signal for sidereal anisotropy in nuclear recoils would support, perhaps more definitively than any other evidence, a discovery claim for a WIMP component of Dark Matter. The speed of earth in galactic coordinates is comparable to the virial velocity of WIMPs in the simplest non-corotating halo model for WIMPs, so a substantial directional anisotropy could be present in WIMP-nuclear collisions. Most contemporary approaches attempt to visualize track images in a low-density TPC. Diffusion during drift and noise in detection limit the mass of these detector concepts to $\sim 100 \mathrm{~g}$. As the active mass needed in the coming era of direct detection searches approaches the ton-scale, the scaling of such low-density TPC approaches to the ton-scale is problematic. Alternatively, a novel concept - based on columnar recombination - may permit sensing of nuclear recoil direction in dense xenon gas; the optimum xenon density may be near $\rho$ $\approx 0.05 \mathrm{~g} / \mathrm{cm}^{3}$, about ten bars or $1.6 \%$ of liquid xenon density, and $\sim 100$ times more dense than possible with track visualization.

The central idea is that, under optimal conditions, the magnitude of columnar recombination may depend sensitively on the angle between track and TPC drift field. The directionality measurement is made, event-by-event, by comparing the recombination and ionization signals. Tracks more closely aligned with field will experience much more recombination, and vice versa. Topology information is extracted prior to drifting the track ionization. Conventional track visualization is unnecessary, and the ionization readout plane can be relatively simple. Restrictions present in contemporary techniques that limit gas density and drift length, and hence total active mass, do not apply. A monolithic room temperature xenon gas Time Projection Chamber approaching the ton-scale, although large, may be feasible.

The recombination signal is prompt UV scintillation at $\sim 300 \mathrm{~nm}$, generated by a unique molecular additive, trimethylamine (TMA). TMA provides four functions that improve directional sensitivity: 1. Penning effect to convert otherwise useless excitations to more ionization; 2) cooling of electrons, which promotes recombination; 3) neutralization of xenon ions by charge exchange, leading to transformation of the event from xenon to TMA ions and electrons; 4) efficient fluorescence in a band centered near $300 \mathrm{~nm}$, providentially matched to wavelength shifting plastic (WLS). The interior of the detector is covered $100 \%$ in WLS plastic to maximize optical sensitivity, which may fall in the range of $10-20 \%$. Even though the detector is physically large, the number of photomultipliers needed for the WLS plastic is small, less than 100. Remarkably, the desired operating conditions for $0-v \beta \beta{ }^{136} \mathrm{Xe}$ experiment may be identical. Effort is beginning at FNAL, LBNL, and ANL to pursue the determination of optimum operating conditions and scientific reach. 


\title{
Solid Xenon R\&D at Fermilab
}

\author{
Jonghee Yoo
}

The solid (crystalline) phase of xenon inherits most of the advantages of using liquid xenon as a detector target material for low energy particles; transparency, self-shielding, absence of intrinsic background, and ionization drift. In the solid phase, even more scintillation light yield $(\sim 60 / \mathrm{keV})$ is reported compared to the liquid phase $(\sim 40 / \mathrm{keV})$. Operation at sub-Kelvin temperature is natural for the solid phase, using superconducting sensors to read out photon, ionization, and phonon signals.

A solar axion search represents a unique physics case for using the crystalline phase of xenon. Solar axions, which may be created in the core region of the Sun, can be detected with a terrestrial $\mathrm{X}$-ray detector. The axion-photon conversion by the strong Coulomb field of atoms in the crystalline $\mathrm{X}$-ray detector may cause coherent Bragg scattering of the photons, which depends on the energy and incident angle of the axions. This strong correlation provides excellent sensitivities below an axion mass of $10 \mathrm{eV}$. This technique can cover QCD axion parameter space that are not accessible by other experiments.

The other strong physics case for using solid xenon is searching for $0 \nu 2 \beta$ decay. The ${ }^{136} \mathrm{Xe}$ enriched solid phase volume, if phonon readout is demonstrated, is a superior detector for a $0 \nu 2 \beta$ experiment. The irreducible background of this experiment is the continuous spectrum of the two neutrino double beta $(2 \nu 2 \beta)$ decay, where the long tail of the spectrum extends up to the $0 \nu 2 \beta$ peak. Therefore, energy resolution is the most important detector parameter that needs to be substantially improved regarding the sensitivity of $0 \nu 2 \beta$ decay signals. The number of phonon quanta being created at the energy deposit of $2.3 \mathrm{MeV}$ is about $10^{7}$ phonons. Hence a $0.3 \%$ energy resolution can be achieved assuming a phonon collection efficieny of $1 \%$. This is about an order of magnitude better compared to the energy resolution at the same energy of the Enriched Xenon double-beta decay Observatory (EXO), $\sigma=2.5 \%$ at $2.3 \mathrm{MeV}$, which uses liquid xenon for the scintillation light and/or ionization signal readout. Therefore, once the phonon signal readout is guaranteed, the solid phase is the best strategy of using the ${ }^{136} \mathrm{Xe}$ for the $0 \nu 2 \beta$ decay search.

Most of the properties of solid xenon have been measured in a small volume or in thin film in the early 1970s through 1990s, but there were no systematic studies successfully carried out with large scale solid xenon. There are two major R\&D issues that need to be addressed in order to make a solid xenon particle detector; the demonstration of the scalability of solid (or crystallin) xenon and the capability to readout signals from solid xenon. The first R\&D phase of the solid xenon project, growing approximately a kilogram of transparent solid phase of xenon, was successfully completed in January 2010 at Fermilab. The second phase R\&D has been started at Fermilab for scintillation light read out and electron drift in the solid xenon. These signal readout $R \& D$ s are expected to be completed in 2013.

There are two remaining challenges for advanced detector development. (1) For solar axion search, single-crystal of xenon is favorable. The University of Enlargen (Germany) is plan to carry out the crystallography study of the solid xenon. (2) For $0 \nu 2 \beta$ study, phonon read out from the solid xenon is favorable which requires mili-kelvin facility with superconducting phonon sensor technology. Characteristics of xenon in milli-kelvin configuration has been rarely studied and deserves further R\&D. 


\title{
4.2 Ultra High Energy Neutrinos
}

\author{
(Author: Peter Gorham, Univ. of Hawaii at Manoa)
}

\subsubsection{Physics Motivation}

Ultra-high energy neutrinos are guaranteed to be present in our universe at a flux level that is determined by known physics but as yet unknown astrophysics. Ultra-high energy cosmic rays have been observed for more than 50 years at energies where they must be already subject to collisions with the cosmic microwave background radiation, a process first elucidated in the 1960s by Greisen, Zatsepin, and Kuzmin. The by-products of such collisions yield the so-called cosmogenic UHE neutrinos, and their flux is a direct measure of the nature and cosmic evolution of the still unidentified - highest energy particle hyper-accelerators in our universe. These accelerators are known to achieve energies above $1020 \mathrm{eV}$, seven orders of magnitude above the current LHC.

UHE cosmic rays measure the endpoint of the source evolution function in the current cosmic epoch, but they cannot by themselves determine the sources or their intensity at earlier epochs, because the UHE cosmic rays are absorbed in transit, leaving only the daughter neutrinos to trace out the sources at earlier times. In fact, for all other high-energy astrophysical sources, the current epoch tends to be relatively benign compared to the higher redshift universe, and we have no reason to doubt that a similar behavior will obtain for the UHE cosmic ray sources. Thus UHE neutrino observations are the only feasible way to directly probe the highest energy universe at epochs where it is plausibly at its most intense. Thus detection of UHE neutrinos is a goal of the first rank in high energy astrophysics, and the problem of identifying and characterizing the UHE cosmic ray sources remains one of the most prominent outstanding mysteries in all of astrophysics.

This goal is not just an astrophysics goal. Once determined, the flux of UHE neutrinos will be stable and predictable to a high degree of precision as models become refined and perfected, in some ways analogous what has happened with the cosmic microwave background mapping. With a welldetermined neutrino flux, the possibilities for doing weak interaction physics at 10-100 TeV CM energies on hadronic targets begin to open up, along with flavor physics, and other studies at energies that will not be achievable on Earth in the foreseeable future. Model-independent cross-section measurements are relatively straightforward once the detector scales are large enough, by just considering ratios of event rates from above and below the horizon. Instrumentation needs in this field have been aided by the rapid worldwide development of wireless RF technology, but now that wireless is moving to multi$\mathrm{GHz}$ microwave bands, the development burden of improved low-noise amplifiers, exotic types of radio antennas, and ultra-sensitive coherent triggering systems is falling back on the investigators. Low-power, ultra-fast sampling RF waveform digitizers are a particular need that requires specific sponsor support, because it has only limited commercial utility. And finally, a continuing energetic support of polar science programs, and the infrastructure necessary to support them, is essential to this field. The most remarkable and precious dielectric on Earth at least for purposes of UHE neutrino detection is Antarctic ice, a threatened and limited resource.

\subsubsection{Existing Technical Capabilities and Challenges}

Because of the very low fluxes and interaction cross sections of UHE cosmogenic neutrinos, the natural scale for an observatory-class detector has been coming into definition. While earlier estimates indicated that cubic-km scales could possible reach these fluxes, it is now evident that much larger scales are necessary, with acceptances approaching a Teraton-steradian, or a thousand cubic $\mathrm{km}$ of ice observing half the sky. Optical technologies cannot scale from current detectors such as IceCube, which is optimized for lower energies and higher fluxes. 


\subsubsection{Proposed new Technology}

In the last decade, a new approach, utilizing the Askaryan effect: coherent Cherenkov radio emission from particle cascades, has emerged as the methodology of choice for these UHE energy ranges. In addition, deep polar ice, while an excellent medium for optical detection of Cherenkov light with 100 meter attenuation lengths, and tens of meter scattering lengths, is almost unbelievable clear for radio propagation: with more than a $\mathrm{km}$ attenuation length, and negligible scattering.

A wide range of efforts focused on detection of highly polarized, broadband, impulsive radio events the hallmarks of the Askaryan effect have engendered a series of experiments, primarily in Antarctica, to realize the goal of cosmogenic neutrino detection. These include the ongoing NASA long-duration balloon experiment ANITA, which observes $1 \mathrm{M}$ cubic km of ice during several weeks duration stratospheric balloon flights, and future efforts such as the ExaVolt Antenna (EVA) a large-scale follow-on to ANITA, the Askaryan Radio Array (ARA) which seeks a several hundred square km embedded radio array at the South Pole, and ARIANNA, a similar effort to place radio instruments over a large area on the Ross Ice Shelf. 


\section{Instrumentation Frontier: Direct Detection of WIMPs}

Andrew Sonnenschein (FNAL)

$4 / 12 / 13$

Within the next two decades, direct searches for WIMP dark matter should come to a conclusion. Either a discovery will be made or the most fruitful regions of parameter space will be exhausted, in which case the emergence of irreducible neutrino backgrounds will frustrate further progress. In either case, the field will require the construction of detectors with target masses of order 10 tons, using a variety of target nuclei to cover the spectrum of possible WIMPnucleus couplings. In the event of a discovery, comparison between rates and spectral shapes on multiple targets will be used to measure WIMP properties by separating particle physics effects from astrophysics.

\section{The Ultimate WIMP Direct Detection Experiments?}

The search for WIMP dark matter particles via direct detection of their nuclear scatterings is a rapidly growing field, with more than twenty-five collaborations operating experiments or planning new ones. The expansion of interest in the field has been driven by exciting improvements in instrumentation, which now bring most of the theoretically favored parameter space within the reach of experiments that appear to be practical to build in the next decade.

The sensitivity of these experiments has increased by three orders of magnitude in the previous decade (2003-2013). The main driver of increased sensitivity is the development of a surprisingly diverse set of techniques for the elimination of background events from environmental radioactivity and cosmic rays. Summaries of these techniques and a comprehensive list of experiments are being prepared by the Cosmic Frontier Dark Matter working groups [1]. The field has evolved from early efforts based on simple adoptions of existing gamma ray detector technology to the development of highly specialized devices. As a point of orientation, in the most recent result from the Xenon-100 experiment, two events passing WIMP selection cuts are found, consistent with expected backgrounds, in a $7600 \mathrm{~kg}$-day exposure, setting a limit on the WIMP-nucleon cross section of $2 \times 10^{-45}$ $\mathrm{cm}^{2}$ for spin-independent couplings. This is a very impressive technical achievement considering that a carefully shielded conventional detector, such as a germanium or sodium iodide crystal, would typically measure on the order of 100 events per kgday in same energy region [2].

In the next decade, assuming progress continues as expected, WIMP searches will become limited in sensitivity by irreducible backgrounds from coherent nuclear scattering of atmospheric and solar neutrinos. This will begin to be a problem when exposures on the order of 10 ton-years are reached, with background rates depending on the specific target nucleus, the energy threshold and the mass of the 
WIMP [3]. The detection of neutrino background events will be an important milestone, since further improvements in sensitivity would run only as the square root of exposure, due to the necessity of background subtraction. In the absence of a WIMP discovery, it is doubtful that experiments large enough to make meaningful further progress will be built. An important consideration is the weakening of the physics case for WIMPs to be the dark matter if a discovery is not made before the neutrino backgrounds are encountered. While there is no hard bottom limit to the nuclear scattering cross section, it becomes progressively more difficult to reconcile very low values with larger cross sections for self-annihilation needed to produce the right amount of dark matter by the thermal freeze out mechanism. Since it is the naturalness of early universe WIMP production in the right abundance to be the dark matter that is responsible for the great popularity of these models, the attention of the field is likely to turn elsewhere.

\section{The Need for Multiple WIMP Targets}

An exhaustive search for WIMPs or a campaign to measure the properties of a discovered particle will require more than one target nucleus. In the non-relativistic limit, WIMP-nucleon couplings are usefully classified simply as "spin-dependent" or "spin-independent". For spin-independent couplings, if all nucleons couple to WIMPs in the same way, the total nuclear cross section is enhanced by a factor $A^{2}$ (with A the atomic number), greatly increasing event rates on heavy nuclei, such as $\mathrm{Xe}$ and $\mathrm{Ge}$ or I, relative to lighter nuclei, such as $\mathrm{Na}, \mathrm{F}$ or Si. However, in some models, the proton and neutron contributions to the spin-independent cross section can be different in magnitude or sign, breaking the expected $\mathrm{A}^{2}$ scaling. For spindependent interactions, the coupling is effectively to the net nuclear spin, due to cancellation between opposite spin pairs and can differ depending on whether the net nuclear spin is carried primarily by a residual neutron or proton. As a result, spin-dependent and independent searches are optimized by a different choice of target nuclei. If a discovery is made, measuring the dependence of a signal on the choice of target nucleus will be the only way to discriminate between different types of WIMP. Comparisons of rates on multiple targets are also important for a convincing proof that an excess of events in an individual experiment is due to something other than miss-modeled background. For example, the comparison of rates has played a critical role in recent attempts to understand excess events in the DAMA, COGENT and CRESST experiments and simultaneously explain the lack of events in CDMS and XENON-10/100.

If a WIMP is discovered, measurements the spectrum of deposited energy will be needed to measure its mass. The shape of the spectrum is independent of the underlying particle physics interaction type, due to the non-relativistic speeds of WIMPs bound to galactic halo, but does depend on the WIMP mass, target nucleus mass and the halo speed distribution. The mass and cross section of the WIMP become difficult to measure independently if the WIMP mass is equal to or larger than the target nucleus mass, because the spectrum of a heavy WIMP resembles that of a lighter WIMP with a smaller cross section. Unfortunately, for large target nuclei, such as Xe or I, flattening of the spectrum due to loss of coherence reduces mass resolution. The best single-target mass resolution is achieved for intermediate mass 
nuclei, such as Ar and Ge, but resolution still becomes quite poor for heavy WIMPs. The degeneracy between mass and cross section can be partially broken by combining data on multiple target nuclei [4]. The comparison of energy spectra on multiple targets also plays in important role in reducing dependence on the model chosen for the halo WIMP speed distribution. This distribution is subject to modeling uncertainties, in part because there are no conventional observables giving information about possible dark matter halo substructure. The energy spectrum of WIMPs can be distorted by the presence of tidal streams or other dark matter structure, such as a "dark disk". By combining energy spectra on multiple targets, it will be possible to test competing models for the structure of the halo, a possibility that has been referred to as "WIMP Astronomy" [5]. This comparison has the potential to greatly reduce biases in the WIMP mass and cross section determination due to halo modeling uncertainties.

\section{References}

[1] http://www.snowmass2013.org/tiki-index.php?page=SLAC

[2] E. Aprile et al., "Dark Matter Results from 225 Live Days of XENON100 Data", ArXiv:1207.5988v2.

[3] L. Strigari, New J.Phys. 11 (2009) 105011

[4] M. Pato et al., Phys Rev D83 (2011) 083505, arXiv:1012.3458v2. K. Arisaka et al., ArXiv:1107.1295v3.

[5] A. H.G. Peter, ArXiv:1103.5145v2. Phys. Rev. D83 (2011) 125029. 


\section{the nEXO detector R\&D}

(Smowmass 2013: Instrumentation Frontier --> Sensors/Detector Systems)

Based on the successful EXO-200 campaign (still ongoing), the EXO collaboration has started designing the second generation EXO experiment to search for neutrinoless DBD of Xe-136. The goal is a detector capable of improving the sensitivity for Xe-136 Onu DBD by >x100 and explore the inverted light Majorana neutrino mass hierarchy. Like EXO-200, the new experiment, dubbed $\mathrm{nEXO}$, is a single-phase, 5 ton liquid xenon Time Projection Chamber (TPC) with scintillation readout. The xenon is enriched to $>80 \%$ in the mass -136 isotope. $\mathrm{nEXO}$ is founded on known technology as much as possible and maximizes what we have learned from EXO-200. However, the larger volume, the increased sensitivity for DBD events, and lower background required call for design refinement in the following detector areas:
a. LXe technology
b. VUV xenon scintillation light detection
c. Cold electronics and charge readout
d. In addition, the EXO collaboration is continuing one of its signature, long-term pursuits: identifying the appearance of Ba-136 ions as a feature consequence of Xe-136 DBDs to drastically improve the specificity of DBD events from (mostly) gamma-ray background.

\section{a. Liquid xenon technology $R \& D$}

The design of a ton-scale, single phase liquid xenon nEXO detector builds directly from the EXO-200 experience and technology. The scale-up, relatively straight forward from the cryogenic point of view, is made less trivial by the need to maintain excellent purity of the xenon liquid from electronegative impurities. With a larger mass of liquid and an increased charge drift distance, the requirement charge collection inefficiency becomes more stringent than in EXO-200 if one wants to maintain good energy resolution (i.e. sigma/E 1\% at $Q$ _bb=2.5 MeV) and minimize position-dependent corrections. Just like in EXO-200, nEXO will collect both ionization and scintillation signals and use their anti-correlated event-by-event amplitude to maximize energy resolution. In addition, because the average scintillation light propagation distance is larger than in EXO-200, the possible attenuation of the scintillation signal with distance because of impurities in the liquid xenon, absolutely negligible in EXO-200, might start showing some effect.

The collaboration is undertaking R\&D to significantly increase the xenon recirculation and purification rates. EXO-200 has shown excellent electron lifetime ( 2-3 ms), with a welldesigned xenon recirculation loop. $\mathrm{nEXO}$ requires a larger xenon recirculation pump and a more efficient thermodynamic cycle for the xenon, and R\&D has begun on both fronts. In addition, $R \& D$ efforts are under way to increase the diagnostic sensitivity for impurities in the recirculated gaseous xenon flow, beyond the devices utilized by EXO-200. Finally, nEXO might require active radon filtration from the xenon: a design of a radon trap was ready for EXO-200 and never implemented as radon levels turned out to be sufficiently low. Such a device will be optimized and resized to meet nEXO specifications.

\section{b. Xenon scintillation light detection in LXe}

EXO-200 pioneered the use of a large number ( 500) of silicon Large Area Avalanche PhotoDiodes (LAAPDs) to replace more traditional photomultiplier tubes. LAAPDs display excellent quantum efficiency for the $175 \mathrm{~nm}$ xenon scintillation light, very low mass and intrinsic radioactive contamination. The latter property made it possible to install them very close to the active volume of the EXO-200 detector. $\mathrm{nEXO}$ will need to have larger optical coverage than EXO-200. While LAAPDs are not ruled out for use in $n E X O, R \& D$ has begun to study possibly less expensive alternatives. New generation silicon photomultipliers (SiPMs) with large area and sensitivity to the VUV Xe scintillation light are being developed in collaboration with industry. The R\&D effort foresees running them directly in LXe. Other light sensors on the market might also be considered in this R\&D phase. Due to the importance of collecting scintillation light as efficiently as possible for energy resolution, nEXO is considering a dedicated effort in 
investigating efficient reflectors for the $175 \mathrm{~nm}$ light. PTFE is widely used in xenon (and argon) detectors (also in EXO-200) for this purpose. In addition, EXO-200 has aluminized part of the copper surfaces directly facing the active xenon volume. Further research in reflector layout optimization is foreseen.

\section{c. Cold, in-liquid electronics and novel charge readout}

Arguably one of the most innovative R\&D activities for $n E X O$ is that on novel charge readout schemes and cold front-end electronics.

i. EXO-200 uses a double set of crossed wires for 2D event position reconstruction and charge collection (i.e. event energy measurement). One plane of wires registers an induced signal from the drifting electrons, while the other collects them. Scaling such a design to nEXO size (while remaining within very tight low background specifications) is technically challenging. In addition and more importantly, the superior sensitivity expected of $\mathrm{nEXO}$ with respect to EXO-200 also calls for improved event topology identification to mainly boost the capability to distinguish double beta (or electron) events from those triggered by gamma rays (the main background). Investigation in highly segmented anode readout planes has started.

ii. the front-end electronics of EXO-200 is housed 2 meters away from the TPC, at room temperature outside the cryostat. This design was driven by radioactivity considerations. Long flat kapton cables take the signals out to the readout electronics, through customdesigned, xenon leak tight feedthroughs. This solution becomes less attractive for nEXO: on one hand the channel count (for both charge and scintillation) is greatly increased and with it the complexity of a cable + feedthrough system; on the other, nEXO foresees an external water radiation shield instead of the more compact EXO-200 lead shield, requiring longer cables. The noise characteristics of such cables would not meet nEXO requirements. The EXO collaboration has begun R\&D of a cold front-end and signal processing system. Cryogenic analog front-end holds the promise of substantially improve noise characteristics. This directly translates in lower thresholds in the charge and scintillation signals and, in turn, better energy resolution. Furthermore, the EXO-200 experience teaches us that the ability of identifying small energy depositions greatly improves background identification. Among the main hurdles for realizing cryogenic electronics for nEXO are low power and ultra-low radioactivity requirements.

\section{d. Ba "tagging"}

A goal of the EXO collaboration since its early days, a scheme to detect the appearance of a Ba ion coincident in time and space with a candidate $\mathrm{Onbb}$ event in $\mathrm{LXe}$ is still being pursued. The goal is to develop a device for single Ba ion tagging that can be added to a later phase of the nEXO detector, improving the sensitivity to mass scales in the upper part of the normal hierarchy. The "zero-background" experiment promised by nEXO+Ba tagging could represent the only possible way to fully explore the inverted mass hierarchy of light Majorana neutrinos and start attaching the normal hierarchy region. Ba tagging R\&D in the past $\sim 8$ years has yielded the ability to: i) trap and detect single $\mathrm{Ba}$ ions even in the presence of gases; ii) deposit and measure a few hundred $\mathrm{Ba}$ ions and atoms in solid $\mathrm{Xe}$; iii) deposit single ions on polished surfaces in a vacuum and count such ions/atoms with several percent efficiency by first desorbing and resonantly ionizing them with Ba-specific lasers (a technique known as RIS) and then identifying them by TOF spectroscopy. Current efforts on Ba tagging from a LXe detector are focusing on depositing a controlled number of Ba atoms onto a surface in LXe which in then extracted to an evacuated region above the liquid where RIS spectroscopy can take place (it should be stressed that the TOF Ba identification can be substituted with a fine-tuned ion trap where Ba ions would be detected via optical spectroscopy). This technique and the one in point ii) are being pushed to achieve single ion/atom sensitivity with order 1 efficiency. 
Title: Large Arrays of Air Cherenkov Detectors

Time Frame: Medium \& Long (2015 - 2025)

Physics Justification: Arrays ( one hundred detectors) of imaging atmospheric Cherenkov telescopes (IACTs) such as CTA (Cherenkov Telescope Array) are at the planning stage, as they have become the instrument of choice for high angular resolution - highly sensitive surveys of the sky at very high energies (VHE, $30 \mathrm{GeV}-300 \mathrm{TeV}$ ). A key science goal for particle \& astrophysics at VHE energies is the indirect dark matter search through annihilation or decay of WIMPs. Other fundamental physics topics include the search for signatures of axion-like particles in spectra of extragalactic $\gamma$-ray sources, tests of Lorentz invariance violation, and physics related to particle acceleration mechanisms reaching energies far beyond man-made accelerators. Novel 2-mirror optical designs of imaging air Cherenkov detectors are now under development, and enable the use of much more compact and high-resolution cameras compared to traditional primary-focus IACTs. This enables the use of large numbers of small size photo detectors such as SiPMs or MAPMTs, and requires low-cost readout electronics. Imaging air showers with at the $0.02^{\circ}$ scale can be achieved by either increasing the number of IACTs participating in the stereoscopic reconstruction, and/or through the use of 2-mirror telescopes equipped with high resolution - wide field of view cameras. Substantial cost savings are possible with an array of fewer but better telescopes and are largely driven by the camera cost.

Technical Capabilities: The air Cherenkov technique requires the recording of Cherenkov flashes of 2-10 ns duration and sampling of the pulse shapes is desirable. The use of $>1 \mathrm{GHz}$ sampling, either through a switched capacitor array (ASIC) or a flash-analog-to-digital converter are possible solutions, however, cost and power usage are key considerations for high pixelation compact cameras. Current generation IACTs have $\leq 1,000$ pixels, whereas next generation designs reach up to $\approx 10^{4}$ pixels $\left(0.065^{\circ}\right.$ resolution $)$. Looking further into the future, cameras with $\approx 10^{5}$ pixels $\left(0.02^{\circ}\right.$ resolution $)$ might become viable, allowing one to bring the IACT technique to its full potential. While the cost of the optical design will increase also somewhat, the major cost savings achievable lie in the camera. The typical cost per electronics channel for current generation IACTs is $\$ 1 \mathrm{k}$, the next generation (CTA) is likely to bring the cost to $\sim \$ 100$ per channel, while the future ultimate IACT will require a pixel cost of $\leq \$ 10$.

Technical Requirements: Low cost and low power digitizers are needed to design the best possible angular resolution IACT. Pixels providing GHz sampling with 10s of $\mu$ sec buffer depth, along with photo detectors exhibiting a peak quantum efficiency $(\geq 50 \%)$ in the blue $350 \mathrm{~nm}$, low dead time and low cross-talk are general broad brush target requirements.

Industrial Involvement: photodetectors (SiPMs, MAPMTs, ...) and digitizers (ASICs, FADCs, ...) will ultimately have to be produced by Industry at a reasonable price.

\section{Key Motivations for this Detector R\&D:}

$\checkmark$ Increase physics capabilities of very large arrays of IACTs.

$\checkmark$ Decrease cost of pixels for very high pixelation cameras.

$\checkmark$ Increase QE of photodetectors to save cost on optical structures.

$\checkmark$ Precision timing systems and wireless triggering for array trigger. 


\title{
Applications of Laser Interferometry in Fundamental Physics Experiments
}

\begin{abstract}
Laser interferometry provides a new and uniquely powerful tool to probe certain aspects of physics, such as exquisitely precise differential position measurements, and controlled, coherent macroscopic quantum states of electromagnetic fields. Although developed primarily for gravitational wave studies, they can be extended with additional R\&D to study the fundamental quantum nature of geometry, or interactions of electromagnetic fields with new types of fields, such as axion-like particles.
\end{abstract}

"Particle Physics" and "High Energy Physics" have outgrown their names. The fundamental nature of the physical world depends on relationships between matter, energy, space and time; some of the most interesting aspects of this relationship depend on spatially extended states (that is, waves and not particles, as in neutrino oscillations), while others, most notably cosmic Dark Energy, also depend on physics at the very lowest energy densities.

The advanced technology of laser interferometry developed for gravitational wave research in recent years provides a new set of experimental tools that can be adapted for experiments in fundamental physics. The capabilities include the ability to measure position with extreme (attometer-scale) precision; to prepare and maintain macroscopic (>meter-scale) systems in coherent quantum-mechanical configuration states; and to create large-scale, coherent states of electromagnetic fields, to study their possible interactions with new forms of matter and energy, such as axion-like particles.

A state of light in a laser cavity shapes the modes of the electromagnetic fields in a particular way: they are confined in space by boundary conditions imposed by mirrors, and their states are entangled with the positions of those macroscopic bodies. The particles or "photons" in these systems are extended in more than one spatial dimension, with a shape that can be controlled. Their quantum state can also be squeezed in phase space. These capabilities provide unique tools to study new physics.

As one example, they can be used to constrain quantum effects of space-time arising at the Planck scale, $c t_{P} \equiv \sqrt{\hbar G / c^{3}}=1.616 \times 10^{-35} \mathrm{~m}$. In standard quantum field theory, space-time is self-consistently assumed to behave classically. However, new quantum effects of geometry originating at the Planck scale - from geometrical degrees of freedom not included in standard field theory - could have effects on macroscopic scales.

The possibility of new quantum-geometrical degrees of freedom is suggested from several theoretical directions. Quantum physics is experimentally proven to violate the principle of locality on which classical spacetime is based. Gravitational theory suggests that quantum states of space-time systems have much less information than predicted by quantum field theory, and suggests that space-time and gravity are approximate statistical behaviors of a quantum system with a holographic information content. [1, 2]
A typical uncertainty in wave mechanics, if information about transverse position is transmitted nonlocally with a bandwidth limit, is the scale familiar from diffractionlimited imaging: the geometric mean of inverse bandwidth and apparatus size. For separations on a laboratory scale, a Planck scale frequency limit leads to a transverse uncertainty in position on the order of attometers. Quantum geometry may be detectable as a new source of quantum-geometrical noise in an interferometer that coherently measures the positions of massive bodies in two directions over a macroscopic volume.[3-5] The Fermilab Holometer experiment (E-990), funded largely through an Early Career Award to Aaron Chou at Fermilab, is designed to detect or rule out quantum-geometrical noise with these properties. [6]

Much of the Holometer technology has been developed by LIGO and other projects to measure displacements due to gravitational radiation. The quantum-geometrical measurement however calls for application of the technology in a new experimental design, with new challenges. If holographic noise is found, a significantly expanded experimental program can be pursued to obtain high precision results and map out the spatiotemporal properties of quantum geometry.

Similarly, spatially extended laser cavities can greatly amplify, via quantum coherence, the interaction of electromagnetic fields with new low-mass fields, such as axion-like particles. In this case, laser cavities may be employed together with large magnets to explore mediating interactions with the new particles.

Profound mysteries such as Dark Energy probably involve new physics that is both non-local and low-energy in character - a departure from the field theory framework that will require new kinds of techniques to study. Laser interferometry has already started to show its unique value and should be developed further by the HEP community.

[1] T. Jacobson, Phys. Rev. Lett. 75, 1260 (1995)

[2] E. P. Verlinde, JHEP 1104, 029 (2011)

[3] C. Hogan, arXiv:1208.3703 [quant-ph].

[4] C. J. Hogan, arXiv:1204.5948 [gr-qc].

[5] C. J. Hogan, Phys. Rev. D 85, 064007 (2012)

[6] http://holometer.fnal.gov 